\title{
General Lagrangian Formulation for Higher Spin Fields with Arbitrary Index Symmetry. 2. Fermionic fields
}

\author{
A. REShetNyAK ${ }^{a, b *}$ \\ ${ }^{a}$ Laboratory of Computer-Aided Design of Materials, Institute of \\ Strength Physics and Materials Science, 634021 Tomsk, Russia, \\ ${ }^{b}$ Tomsk State Pedagogical University, 634041 Tomsk, Russia
}

\begin{abstract}
We continue the construction of a Lagrangian description of irreducible half-integer higher-spin representations of the Poincare group with an arbitrary Young tableaux having $k$ rows, on a basis of the BRST-BFV approach suggested for bosonic fields in our first article (Nucl. Phys. B862 (2012) 270, arXiv:1110.5044 hep-th]). Starting from a description of fermionic mixed-symmetry higher-spin fields in a flat space of any dimension in terms of an auxiliary Fock space associated with a special Poincare module, we realize a conversion of the initial operator constraint system (constructed with respect to the relations extracting irreducible Poincare-group representations) into a system of first-class constraints. To do this, we find, in first time, by means of generalized Verma module the auxiliary representations of the constraint subsuperalgebra, to be isomorphic due to Howe duality to $\operatorname{ssp}(1 \mid 2 k)$ superalgebra, and containing the subsystem of second-class constraints in terms of new oscillator variables. We suggest a universal procedure of finding unconstrained gauge-invariant Lagrangians with reducible gauge symmetries, describing the dynamics of both massless and massive fermionic fields of any spin. It is shown that the space of BRST cohomologies with a vanishing ghost number is determined only by constraints corresponding to an irreducible Poincare-group representation. As examples of the general approach, we propose a method of Lagrangian construction for fermionic fields subject to an arbitrary Young tableaux having 3 rows, and obtain a gauge-invariant Lagrangian for a new model of a massless rank-3 spintensor field of spin $(5 / 2,3 / 2)$ with first-stage reducible gauge symmetries and a non-gauge Lagrangian for a massive rank-3 spin-tensor field of spin $(5 / 2,3 / 2)$.
\end{abstract}

Keywords: higher-spin fields; gauge theories; Lagrangian formulation.

\section{Introduction}

Higher-spin field theory, in its various aspects, has been under a long and intense study, in the hopes to re-examine the problems of a unified description for the variety of elementary particles, and to discover new approaches to a unification of the known fundamental interactions. Higherspin field theory is in close relation to superstring theory, which operates an infinite set of bosonic

*reshet@ispms.tsc.ru 
and fermionic fields of various spins, providing the consideration of higher-spin theory as a tool of investigating the structure of superstring theory. For the current progress in higher-spin field theory, see the reviews [1], whereas some recent directions in higher-spin theory, starting from the pioneering papers [2], 3], 4], are examined in [5]-[20].

The dynamics of totally symmetric free higher-spin fields is currently the most well-developed area in the variety of unitary representations of the Poincare and AdS algebras [3], 4], 21, 22]. This situation is due to the fact that a $4 d$ space-time does not admit any mixed-symmetry irreducible representations, except for dual theories. It is well-known that in higher space-time dimensions there arise mixed-symmetry representations, determined by spin-like parameters being more than one in number [23], [24], [25], whereas their field-theoretic description is not so well-developed as for totally symmetric representations. While the simplest mixed-symmetric HS bosonic fields were examined in [26], attempts to construct Lagrangian descriptions of free and interacting higher-spin field theories have met with consistency problems, which have not yet been completely solved. Unconstrained Lagrangians for half-integer HS fields with higher derivatives in the "metric-like" formulation in Minkowski space-time for massless irreducible Poincare group representations, and without higher derivatives in the case of reducible ones, have been derived on a basis of the Bianchi identities resolution in [27], whereas in the case of arbitrary irreducible Poincare group representations with a half-integer spin the resulting unconstrained action (given by Eq. (6.31) in [27]) contains some special projection operators that have not yet been found explicitly, thus making the Lagrangian formulation unclosed, and therefore requires some additional efforts to find them 1 . The main result in the task of a constrained (with off-shell gamma-traceless algebraic constraints) Lagrangian construction for arbitrary massless mixed-symmetry fermionic HS fields in a $d$-dimensional Minkowski space-time has been recently obtained in [29] within the "frame-like" formulation. Meanwhile, in the (anti-)de Sitter case, the same results for massless and massive mixed-symmetry fermionic HS fields in the "frame-like" formulation with off-shell gamma-traceless constraints are known in the case of a Young tableaux with two rows [30].

In this article, which continues our investigation started in 31] for tensor fields (see also Ref. [32]), we construct a gauge-invariant Lagrangian description in the "metric-like" formalism for both massless and massive mixed-symmetry spin-tensor fields of Lorentz rank $n_{1}+n_{2}+\ldots+n_{k}$ and spin $\mathbf{s}=\left(n_{1}+1 / 2, n_{2}+1 / 2, \ldots, n_{k}+1 / 2\right)$, with any integer numbers $n_{1} \geq n_{2} \geq \ldots \geq n_{k} \geq 1$ for $k \leq[(d-1) / 2]$ in a $d$-dimensional Minkowski space, corresponding to a unitary irreducible Poincare-group $I S O(1, d-1)$ representation with a Young tableaux having $k$ rows. In the case of Minkowski space, several approaches have been suggested to study mixed-symmetry higherspin fields [23], [33], [34], [35]. Our approach is based on the BFV-BRST construction [36] (see also the reviews [37, [38]), which was initially developed for a Hamiltonian quantization of dynamical systems subject to first-class constraints. We recall that the application of the BRST construction to higher-spin field theory in constant-curvature spaces consists of four steps, being reduced to three steps in the case of flat spaces. At the first stage, the conditions determining representations with a given spin and mass are regarded as a system of mixed-class operator constraints in an auxiliary Fock space. Second, the system of initial constraints is converted, with a preservation of the initial algebraic structure, into a system of first-class constraints only, acting in an enlarged Fock space, with respect to which one constructs a BRST operator (being the (nontrivial) third step in the case of fields in AdS spaces). Finally, a Lagrangian for a higherspin field is constructed in terms of the BRST operator, in such a way that the corresponding equations of motion reproduce the initial constraints. It should be emphasized that this approach automatically implies a gauge-invariant Lagrangian description, reflecting the general fact of BV-

\footnotetext{
${ }^{1}$ In [31, we analyze in Footnote 2 the same problem of an unconstrained Lagrangian description for bosonic fields in a flat space of any dimension, subject to an arbitrary Young tableaux in [28, where the projectors $\Pi_{k l m}^{i j k}$ in the action given by Eq. (5.25) have a determined status only in the case of totally symmetric fields, see Eq.(5.28).
} 
BFV duality [39], [40], [41], realized in order to reproduce a Lagrangian action by means of a Hamiltonian object.

The development of flat dynamics for mixed-symmetry gauge fields has been examined in [42], [43], 44], 45], [46] for massless bosonic higher-spin fields with two rows of the Young tableaux [47], and recently also for interacting bosonic higher-spin fields [48, [49, [50]. Lagrangian descriptions of massless mixed-symmetry fermionic and bosonic higher-spin fields in (A)dS spaces have been suggested within a "frame-like" approach in [51, whereas for massive fields of lower superspins in flat and (A)dS spaces they have been examined in [52]. In turn, the aspects of interacting higher-spin fields and its relation to string theory spectrum was considered in [53]. For the sake of completeness, we emphasize that in the case of free totally symmetric higher-spin fields of half-integer spin the BRST approach has been used to obtain Lagrangians in the flat space [55] and in the (A)dS space [56], whereas for totally symmetric bosonic HS tensors in AdS spaces the same has been done in [57, and for mixed-symmetric ones, subject to a Young tableaux with two rows, in 58 .

The paper is organized as follows. In Section 2, we present a closed superalgebra of operators (using the Howe duality), based on constraints in an auxiliary Fock space with a symmetric basis, that determines a massless irreducible representation of the Poincare group in $\mathbb{R}^{1, d-1}$ with a generalized spin $\mathbf{s}=\left(n_{1}+\frac{1}{2}, \ldots, n_{k}+\frac{1}{2}\right)$. In Section 3, we construct an auxiliary representation for the rank- $\left(\left[\frac{(d-1)}{2}\right],\left[\frac{(d-1)}{2}\right]\right)$ orthosymplectic $\operatorname{osp}(1 \mid 2 k)$ subsuperalgebra of the superalgebra of initial constraints, corresponding to the subsystem of second-class constraints in terms of new (additional) creation and annihilation operators in Fock space 2 . As a result, the initial system of first- and second-class odd and even constraints is converted into a system of first-class constraints in the space being the tensor product of the initial and new Fock spaces. Next, we construct the standard BRST operator for the converted constraint superalgebra in Section 4. The construction of an action and of a sequence of reducible gauge transformations describing the propagation of a mixed-symmetry fermionic field of an arbitrary spin is realized in Section 5. We show, after applying dimensional reduction to the massless half-integer mixed-symmetry HS field in a $(d+1)$ dimensional flat space, that the Lagrangian description for a massive HS field in a $d$-dimensional Minkowski space of the same type is deduced. In Section 6, we demonstrate that the general procedure contains, first, a previously known algorithm of Lagrangian construction for fermionic fields subject to a Young tableaux with two rows, and, second, a new algorithm for spin-tensor fields with three rows in the corresponding Young tableaux. In Subsections 6.3, 6.4, we construct new unconstrained Lagrangian descriptions for both third-rank massless and massive spin-tensor fields with spin $(5 / 2,3 / 2)$, which have not been obtained before. In Conclusion, we summarize the results of this work and outline some open problems. Finally, in Appendix A we construct an auxiliary representation for the $\operatorname{sep}(1 \mid 2 k)$ algebra on a basis of the (generalized) Verma module, shortly described in Appendix A.2. Appendix B is devoted to finding a polynomial representation of the operator superalgebra given by Tables 1, 2 in terms of creation and annihilation operators. In Appendix C, we prove the fact that the constructed general Lagrangian actually reproduces correct conditions for the field that determine an irreducible representation of the Poincare group; we also suggest a new form of gauge-fixing procedure. In Appendix D, the expressions for the field and all the Fock-space gauge vectors are presented in the powers of ghost creation operators, to be applied to a Lagrangian construction for the third-rank Dirac spin-tensor.

In addition to the conventions of [31], [47], [55], [64], we use, first, the mostly minus signature for the metric tensor $\eta_{\mu \nu}=\operatorname{diag}(+,-, \ldots,-)$, with Lorentz indices $\mu, \nu=0,1, \ldots, d-1$, second, the relations $\left\{\gamma^{\mu}, \gamma^{\nu}\right\}=2 \eta^{\mu \nu}$ for the Dirac matrices $\gamma^{\mu}$, third, the notation $\varepsilon(A), g h(A)$ for the

${ }^{2}$ Notice that a similar construction for fermionic HS fields subject to a Young tableaux with 2 rows in a flat space has been presented in [59. 
respective values of Grassmann parity and ghost number of a quantity $A$, and denote by $[A, B\}$ the supercommutator of quantities $A, B$, which, in case they possess definite values of Grassmann parity, is given by $[A, B\}=A B-(-1)^{\varepsilon(A) \varepsilon(B)} B A$.

\section{Derivation of Half-Integer HS Symmetry Superalgebra in $\mathbb{R}^{1, d-1}$}

Let us study a massless half-integer irreducible representation of the Poincare group in a $d$ dimensional Minkowski space, which is to be described by a spin-tensor field: $\Psi_{\left(\mu^{1}\right)_{n_{1}},\left(\mu^{2}\right)_{n_{2}}, \ldots,\left(\mu^{k}\right)_{n_{k}}}$ $\equiv \Psi_{\mu_{1}^{1} \ldots \mu_{n_{1}}^{1}, \mu_{1}^{2} \ldots \mu_{n_{2}}^{2}, \ldots, \mu_{1}^{k} \ldots \mu_{n_{k}}^{k}{ }_{A}}(x)$, with the Dirac index $A$ (being suppressed in what follows) of rank $\sum_{i \geq 1}^{k} n_{i}$ and the generalized spin $\mathbf{s}=\left(n_{1}+1 / 2, n_{2}+1 / 2, \ldots, n_{k}+1 / 2\right)\left(n_{1} \geq n_{2} \geq \ldots \geq n_{k}>\right.$ $0, k \leq[(d-1) / 2])$, which corresponds to a Young tableaux with $k$ rows of length $n_{1}, n_{2}, \ldots, n_{k}$, respectively,

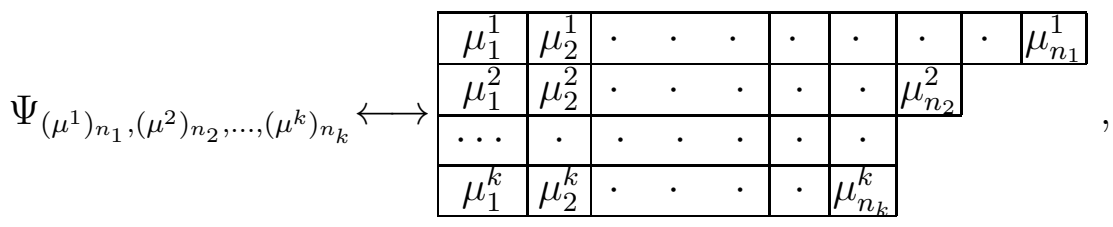

The field is symmetric with respect to permutations of each type of Lorentz indices $\mu^{i}$, and obeys the Dirac equations (2.2), as well as those of gamma-tracelessness (2.3) and mixed-symmetry (2.4) $\left[\right.$ for $\left.i, j=1, \ldots, k ; l_{i}, m_{i}=1, \ldots, n_{i}\right]$,

$$
\begin{aligned}
& \imath \gamma^{\mu} \partial_{\mu} \Psi_{\left(\mu^{1}\right)_{n_{1}},\left(\mu^{2}\right)_{n_{2}}, \ldots,\left(\mu^{k}\right)_{n_{k}}}=0, \\
& \gamma^{\mu_{l_{i}}^{i}} \Psi_{\left(\mu^{1}\right)_{n_{1}},\left(\mu^{2}\right)_{n_{2}}, \ldots,\left(\mu^{k}\right)_{n_{k}}}=0 \text {, } \\
& \Psi_{\left(\mu^{1}\right)_{n_{1}}, \ldots,\{\left(\mu^{i}\right)_{n_{i}}, \ldots, \underbrace{j}_{1} \ldots \mu_{l_{j}}^{j}\} \ldots \mu_{n_{j}}^{j}, \ldots\left(\mu^{k}\right)_{n_{k}}}=0, \quad i<j, \quad 1 \leq l_{j} \leq n_{j} \text {, }
\end{aligned}
$$

where the figure bracket below denotes that the indices included in it do not take part in symmetrization, which thus concerns only the indices $\left(\mu^{i}\right)_{n_{i}}, \mu_{l_{j}}^{j}$ in $\{\left(\mu^{i}\right)_{n_{i}} \underbrace{\ldots, \mu_{1}^{j} \ldots} \mu_{l_{j}}^{j}\}$.

All irreducible representations can be described simultaneously if we make one of the two choices of introducing an auxiliary Fock space $\mathcal{H}$. We consider, as usual, the Fock space $\mathcal{H}$ generated by the bosonic (the case of a symmetric basis) creation and annihilation operators $a_{\mu^{i}}^{i+}, a_{\nu^{j}}^{j}$ with additional internal indices, $i, j=1, \ldots, k, \mu^{i}, \nu^{j}=0,1 \ldots, d-13^{3}$ :

$$
\left[a_{\mu^{i}}^{i}, a_{\nu^{j}}^{j+}\right]=-\eta_{\mu^{i} \nu^{j}} \delta^{i j}, \quad \delta^{i j}=\operatorname{diag}(1,1, \ldots 1)
$$

The general state of the Fock space is a Dirac-like spinor, having the form

$$
|\Psi\rangle=\sum_{n_{1}=0}^{\infty} \sum_{n_{2}=0}^{n_{1}} \cdots \sum_{n_{k}=0}^{n_{k-1}} \Psi_{\left(\mu^{1}\right)_{n_{1}},\left(\mu^{2}\right)_{n_{2}}, \ldots,\left(\mu^{k}\right)_{n_{k}}} \prod_{i=1}^{k} \prod_{l_{i}=1}^{n_{i}} a_{i}^{+\mu_{l_{i}}^{i}}|0\rangle,
$$

which provides the symmetry property of $\Psi_{\left(\mu^{1}\right)_{n_{1}},\left(\mu^{2}\right)_{n_{2}}, \ldots,\left(\mu^{k}\right)_{n_{k}}}$ with respect to permutations of indices of the same type. Using the accepted terminology, we refer to the vector (2.6) as the basic

\footnotetext{
${ }^{3}$ There exists another realization of all irreps be means of a different Fock space $\mathcal{H}^{\text {as }}$, generated by fermionic oscillators (antisymmetric basis) $\hat{a}_{\mu^{m}}^{m}(x), \hat{a}_{\nu^{n}}^{\hat{n}+}(x)$ with the anticommutation relations $\left\{\hat{a}_{\mu^{m}}^{m}, \hat{a}_{\nu^{n}}^{n+}\right\}=-\eta_{\mu^{m}} \nu^{n} \delta^{m n}$, for $m, n=1, \ldots, n_{1}$, and one can complete the below procedure, which follows the prescription of [60] for totally antisymmetric spin-tensors for $n_{1}=n_{2}=\ldots=n_{k}$.
} 
vectorn.

Because of the property of translational invariance of the vacuum, $\partial_{\mu}|0\rangle=0$, the conditions (2.2) -(2.4) can be equivalently expressed in terms of the bosonic operators

$$
\begin{aligned}
& \tilde{t}_{0}=-i \gamma^{\mu} \partial_{\mu}, \quad \tilde{t}^{i}=\gamma^{\mu} a_{\mu}^{i}, \\
& t^{i_{1} j_{1}}=a_{\mu}^{i_{1}+} a^{j_{1} \mu}, \quad i_{1}<j_{1}
\end{aligned}
$$

as follows:

$$
\tilde{t}_{0}|\Psi\rangle=\tilde{t}^{i}|\Psi\rangle=t^{i_{1} j_{1}}|\Psi\rangle=0 .
$$

Thus, the set of $\left(\frac{1}{2} k(k+1)+1\right)$ primary constraints (2.9) with $\left\{o_{\alpha}\right\}=\left\{\tilde{t}_{0}, \tilde{t}^{i}, t^{i_{1} j_{1}}\right\}$ for each component $\Psi_{\left(\mu^{1}\right)_{n_{1}},\left(\mu^{2}\right)_{n_{2}}, \ldots,\left(\mu^{k}\right)_{n_{k}}}$ of the vector (2.6) is equivalent to Eqs. (2.2) -(2.4) for all values of spin subject to the condition $n_{1} \geq n_{2} \geq \ldots \geq n_{k}>0$. In turn, if we impose, in addition to Eqs.(2.9), the constraints

$$
g_{0}^{i}|\Psi\rangle=\left(n_{i}+\frac{d}{2}\right)|\Psi\rangle,
$$

then these combined conditions are in one-to-one correspondence with Eqs. (2.2)-(2.4) for the spin-tensor $\Psi_{\left(\mu^{1}\right)_{n_{1}},\left(\mu^{2}\right)_{n_{2}}, \ldots,\left(\mu^{k}\right)_{n_{k}}}$ with a given value of spin $\mathbf{s}=\left(n_{1}+\frac{1}{2}, n_{2}+\frac{1}{2}, \ldots, n_{k}+\frac{1}{2}\right)$.

Because of the fermionic nature of equations (2.2) -(2.4) with respect to the standard Lorentzlike Grassmann parity, and due to the bosonic nature of the primary constraint operators $\tilde{t}_{0}, \tilde{t}^{i}$, $\varepsilon\left(\tilde{t}_{0}\right)=\varepsilon\left(\tilde{t}^{i}\right)=0$, in order to equivalently transform these operators into fermionic ones 5 , we follow Refs. [55], [56] and introduce a set of $d+1$ Grassmann-odd gamma-matrix-like objects $\tilde{\gamma}^{\mu}$, $\tilde{\gamma}$, subject to the conditions

$$
\left\{\tilde{\gamma}^{\mu}, \tilde{\gamma}^{\nu}\right\}=2 \eta^{\mu \nu}, \quad\left\{\tilde{\gamma}^{\mu}, \tilde{\gamma}\right\}=0, \quad \tilde{\gamma}^{2}=-1
$$

and related to the conventional gamma-matrices as follows:

$$
\gamma^{\mu}=\tilde{\gamma}^{\mu} \tilde{\gamma}
$$

We can now define Grassmann-odd constraints,

$$
t_{0}=-\imath \tilde{\gamma}^{\mu} \partial_{\mu}, \quad t^{i}=\tilde{\gamma}^{\mu} a_{\mu}^{i},
$$

related to the operators (2.7) as follows:

$$
\left(t_{0}, t^{i}\right)=\tilde{\gamma}\left(\tilde{t}_{0}, \tilde{t}^{i}\right)
$$

An essential part of the procedure of Lagrangian description concerns the property of BFVBRST operator $Q, Q=C^{\alpha} o_{\alpha}+$ more, to be Hermitian, which is equivalent to the requirements $\left\{o_{\alpha}\right\}^{+}=\left\{o_{\alpha}\right\}$ and closedness for $\left\{o_{\alpha}\right\}$ with respect to the supercommutator multiplication $[$,$\} .$ It is evident that the set of $\left\{o_{\alpha}\right\}$ violates the above conditions. To provide them, we follow the

\footnotetext{
${ }^{4}$ One may regard the set of all finite Dirac-like vectors with finite upper limits for $n_{1}$ and different choices of spin $\mathbf{s}$ as a vector space of polynomials $P_{k}^{d}\left(a^{+}\right)$, being Dirac-like spinors in powers of $a_{i}^{+\mu^{i}}$. The Lorentz algebra is realized by the action on $P_{k}^{d}\left(a^{+}\right)$of Lorentz transformations, $M^{\mu \nu}=\sum_{i \geq 1}^{k} a_{i}^{+[\mu} a^{\nu] i}+\frac{1}{2} \gamma_{\mu \nu}$, with the standard rule $A^{[\mu} B^{\nu]} \equiv A^{\mu} B^{\nu}-A^{\nu} B^{\mu}$ and $\gamma_{\mu \nu}=\frac{1}{2} \gamma_{[\mu} \gamma_{\nu]}$.

${ }^{5}$ Indeed, the relations $\tilde{t}^{i} \tilde{t}^{i}=\frac{1}{2} \gamma^{\mu} \gamma^{\nu}\left(a_{\mu}^{i} a_{\nu}^{i}+a_{\nu}^{i} a_{\mu}^{i}\right)=\frac{1}{2}\left\{\gamma^{\mu}, \gamma^{\nu}\right\} a_{\mu}^{i} a_{\nu}^{i}=2 l^{i i}$, see Eqs. (2.16), imply the validity of the anticommutator representation $\left\{\tilde{t}^{i}, \tilde{t}^{i}\right\}=4 l^{i i}$, however, in the case of a bosonic $\tilde{t}^{i}$ this is contradictory from the viewpoint of the spin-statistic theorem.
} 
case of totally-symmetric [55] and mixed-symmetry spin-tensors with $Y\left(n_{1}, n_{2}\right)$ [59], and define an odd scalar product in $\mathcal{H}$,

$$
\begin{aligned}
\langle\tilde{\Phi} \mid \Psi\rangle= & \int d^{d} x \sum_{n_{1}=0}^{\infty} \sum_{n_{2}=0}^{n_{1}} \ldots \sum_{n_{k}=0}^{n_{k-1}} \sum_{p_{1}=0}^{\infty} \sum_{p_{2}=0}^{p_{1}} \ldots \sum_{p_{k}=0}^{p_{k-1}}\langle 0| \prod_{j=1}^{k} \prod_{m_{j}=1}^{p_{j}} a_{j}^{\nu_{m_{j}}^{j}} \Phi_{\left(\nu^{1}\right)_{p_{1}},\left(\nu^{2}\right)_{p_{2}}, \ldots,\left(\nu^{k}\right)_{p_{k}}}^{+}(x) \\
& \times \tilde{\gamma}_{0} \Psi_{\left(\mu^{1}\right)_{n_{1}},\left(\mu^{2}\right)_{n_{2}}, \ldots,\left(\mu^{k}\right)_{n_{k}}}(x) \prod_{i=1}^{k} \prod_{l_{i}=1}^{n_{i}} a_{i}^{+\mu_{l_{i}}^{i}}|0\rangle
\end{aligned}
$$

for nonnegative integers $n_{i}, p_{j}$. As a result, the set of primary constraints $\left\{o_{\alpha}\right\}$, being extended, first, due to the closedness condition by means of the D'Alembert operator, and the divergentless and traceless operators

$$
l_{0}=\partial^{\mu} \partial_{\mu}, \quad l^{i}=-i a_{\mu}^{i} \partial^{\mu}, \quad l^{i j}=\frac{1}{2} a^{i \mu} a_{\mu}^{j}
$$

second, due to the Hermitian conjugation properties by the operators

$$
\left(t^{i+}, l^{i+}, l^{i j+}, t^{i_{1} j_{1}+}\right)=\left(\tilde{\gamma}^{\mu} a_{\mu}^{i+},-i a_{\mu}^{i+} \partial^{\mu}, \frac{1}{2} a_{\mu}^{i+} a^{j \mu+}, a_{\mu}^{i_{1}} a^{j_{1} \mu+}\right), i \leq j ; i_{1}<j_{1},
$$

satisfies both requirements if the particle number operators $g_{0}^{i}$ are included in the set of all constraints $o_{I}$, thus having the structure

$$
\left\{o_{I}\right\}=\left\{o_{\alpha}, o_{\alpha}^{+} ; g_{0}^{i}\right\} \equiv\left\{o_{a}, o_{a}^{+} ; t_{0}, l_{0}, l^{i}, l^{i+} ; g_{0}^{i}\right\}
$$

Being combined, the set $\left\{o_{a}, o_{a}^{+}\right\}$in Eq. (2.18), for $\left\{o_{a}\right\}=\left\{t^{i}, l^{i j}, t^{i_{1} j_{1}}\right\}$, and the one $\left\{o_{A}\right\}=$ $\left\{t_{0}, l_{0}, l^{i}, l^{i+}\right\}$, may be interpreted in the Hamiltonian analysis of dynamical systems as respective operator-valued $2 k^{2}$ bosonic and $2 k$ fermionic second-class, as well as $(2 k+1)$ bosonic and 1 fermionic first-class constraint subsystems among $\left\{o_{I}\right\}$ for a topological gauge system (one with the zero Hamiltonian), since

$$
\left[o_{a}, o_{b}^{+}\right\}=f_{a b}^{c} o_{c}+\Delta_{a b}\left(g_{0}^{i}\right),\left[o_{A}, o_{B}\right\}=f_{A B}^{C} o_{C},\left[o_{a}, o_{B}\right\}=f_{a B}^{C} o_{C}
$$

Here, the constants $f_{a b}^{c}, f_{A B}^{C}, f_{a B}^{C}$ possess the generalized antisymmetry property with respect to permutations of lower indices, whereas the quantities $\Delta_{a b}\left(g_{0}^{i}\right)$ form a non-degenerate $\left(k \times k ; k^{2} \times k^{2}\right)$ supermatrix, $\left\|\Delta_{a b}\right\|$, in the Fock space $\mathcal{H}$ on the surface $\Sigma \subset \mathcal{H}:\left\|\Delta_{a b}\right\|_{\mid \Sigma} \neq 0$, which is determined by the equations $\left(o_{a}, t_{0}, l_{0}, l^{i}\right)|\Psi\rangle=0$. The set of $o_{I}$ contains the operators $g_{0}^{i}$, which are not constraints in $\mathcal{H}$, due to Eqs. (2.10).

Explicitly, the operators $o_{I}$ obey a Lie superalgebra with the commutation relations

$$
\left[o_{I}, o_{J}\right\}=f_{I J}^{K} o_{K}, f_{I J}^{K}=-(-1)^{\varepsilon\left(o_{I}\right) \varepsilon\left(o_{J}\right)} f_{J I}^{K},
$$

where the structure constants $f_{I J}^{K}$ used in Eq.(2.19]) include the constants $f_{a b}^{\left[g_{0}^{i}\right]}: f_{a b}^{\left[g_{0}^{i}\right]} g_{0}^{i} \equiv \Delta_{a b}^{\left[g_{0}^{i}\right]}\left(g_{0}^{i}\right)$ and are determined by Multiplication Table1, with commutators only, and Multiplication Table 2 , composed from anticommutators of fermionic constraints only.

First, note that in Table 1, except for the first three rows with the fermionic constraints $t_{0}, t_{i_{2}}, t_{i_{2}}^{+}$, described in the case of an integer HS symmetry algebra $\mathcal{A}\left(Y(k), \mathbb{R}^{1, d-1}\right)$ in 31$]$, the operators $t^{i_{2} j_{2}}, t_{i_{2} j_{2}}^{+}$obey (by definition) the properties

$$
\left(t^{i_{2} j_{2}}, t_{i_{2} j_{2}}^{+}\right) \equiv\left(t^{i_{2} j_{2}}, t_{i_{2} j_{2}}^{+}\right) \theta^{j_{2} i_{2}}, \theta^{j_{2} i_{2}}=1(0) \text { for } j_{2}>i_{2}\left(j_{2} \leq i_{2}\right)
$$

with the Heaviside $\theta$-symbol $\theta^{j i}$ and without summation with respect to the indices $i_{2}, j_{2}$. The figure brackets for the indices $i_{1}, i_{2}$ in the quantity $A^{\left\{i_{1}\right.} B^{\left.i_{2}\right\} i_{3}} \theta^{\left.i_{3} i_{2}\right\}}$ imply the symmetrization 


\begin{tabular}{|c|c|c|c|c|c|c|c|c|}
\hline$[\downarrow, \rightarrow]$ & $\overline{t^{i_{1} j_{1}}}$ & $t_{i_{1} j_{1}}^{+}$ & $l_{0}$ & $l^{i}$ & $l^{i+}$ & $\bar{l} l^{i_{1} j_{1}}$ & 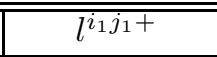 & $g_{0}^{i}$ \\
\hline$\overline{t_{t_{0}}}$ & $\overline{0}$ & 0 & 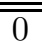 & $\overline{0}$ & 0 & $\overline{0}$ & $\overline{0}$ & 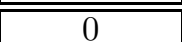 \\
\hline$t^{i_{2}}$ & $-t^{j_{1}} \delta^{i_{2} i_{1}}$ & $-t_{i_{1}} \delta^{i_{2}}{ }_{j_{1}}$ & 0 & 0 & $-t_{0} \delta^{i_{2} i}$ & 0 & $-\frac{1}{2} t^{\left\{i_{1}+\right.} \delta^{\left.j_{1}\right\} i_{2}}$ & $t^{i_{2}} \delta^{i_{2} i}$ \\
\hline$t^{i_{2}+}$ & $t^{i_{1}+} \delta^{i_{2} j_{1}}$ & $t_{j_{1}}^{+} \delta_{i_{1}}^{i_{2}}$ & 0 & $t_{0} \delta^{i_{2} i}$ & 0 & $\frac{1}{2} t^{\left\{i_{1}\right.} \delta^{\left.j_{1}\right\} i_{2}}$ & 0 & $-t^{i_{2}+} \delta^{i_{2} i}$ \\
\hline$t^{i_{2} j_{2}}$ & $A^{i_{2} j_{2}, i_{1} j_{1}}$ & $\overline{B^{i_{2} j_{2}}{ }_{i_{1} j_{1}}}$ & 0 & 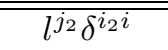 & $-l^{i_{2}+} \delta^{j_{2} i}$ & $l^{\left\{j_{1} j_{2}\right.} \delta^{\left.i_{1}\right\} i_{2}}$ & $-l^{i_{2}\left\{i_{1}+\right.} \delta^{\left.j_{1}\right\} j_{2}}$ & $F^{i_{2} j_{2}, i}$ \\
\hline$t_{i_{2} j_{2}}^{+}$ & $-B^{i_{1} j_{1}} i_{2} j_{2}$ & $A_{i_{1} j_{1}, i_{2} j_{2}}^{+}$ & 0 & $l_{i_{2}} \delta_{j_{2}}^{i}$ & $-l_{j_{2}}^{+} \delta_{i_{2}}^{i}$ & $l_{i_{2}}{ }^{\left\{j_{1}\right.} \delta_{j_{2}}^{\left.i_{1}\right\}}$ & $-l_{j_{2}}\left\{j_{1}+\delta_{i_{2}}^{\left.i_{1}\right\}}\right.$ & $-F_{i_{2} j_{2}}{ }^{i+}$ \\
\hline$l_{0}$ & 0 & 0 & 0 & 0 & 0 & 0 & 0 & 0 \\
\hline$l^{j}$ & $-l^{j_{1}} \delta^{i_{1} j}$ & $-l_{i_{1}} \delta_{j_{1}}^{J}$ & 0 & 0 & $l_{0} \delta^{j i}$ & 0 & $-\frac{1}{2} l\left\{i_{1}+\delta^{\left.j_{1}\right\} j}\right.$ & $l^{j} \delta^{i j}$ \\
\hline$l^{j+}$ & $l^{i_{1}+} \delta^{j_{1} j}$ & $l_{j_{1}}^{+} \delta_{i_{1}}^{J}$ & 0 & $-l_{0} \delta^{j i}$ & 0 & $\frac{1}{2} l^{\left\{i_{1}\right.} \delta^{\left.j_{1}\right\} j}$ & 0 & $-l^{j+} \delta^{i j}$ \\
\hline$l^{i_{2} j_{2}}$ & $-l^{j_{1}\left\{j_{2}\right.} \delta^{\left.i_{2}\right\} i_{1}}$ & $-l_{i_{1}}\left\{i_{2}+\delta_{j_{1}}^{\left.j_{2}\right\}}\right.$ & 0 & 0 & $-\frac{1}{2} l^{\left\{i_{2}\right.} \delta^{\left.j_{2}\right\} i}$ & 0 & $L^{i_{2} j_{2}, i_{1} j_{1}}$ & $l^{i\left\{i_{2}\right.} \delta^{\left.j_{2}\right\} i}$ \\
\hline$l^{i_{2} j_{2}+}$ & $l^{i_{1}\left\{i_{2}+\right.} \delta^{\left.j_{2}\right\} j_{1}}$ & $l_{j_{1}}\left\{j_{2}+\delta_{i_{1}}^{\left.i_{2}\right\}}\right.$ & 0 & $\frac{1}{2} l^{\left\{i_{2}+\right.} \delta^{\left.i j_{2}\right\}}$ & 0 & $-L^{i_{1} j_{1}, i_{2} j_{2}}$ & 0 & $-l^{i\left\{i_{2}+\right.} \delta^{\left.j_{2}\right\} i}$ \\
\hline$g_{0}^{j}$ & $-F^{i_{1} j_{1}, j}$ & 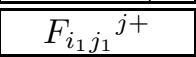 & 0 & $-l^{i} \delta^{i j}$ & $\overline{l^{i+} \delta^{i j}}$ & 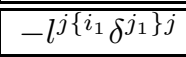 & $\bar{l} l^{j\left\{i_{1}+\delta^{j_{1}}\right\} j}$ & 0 \\
\hline
\end{tabular}

Table 1: even-even and odd-even parts of HS symmetry superalgebra $\mathcal{A}^{f}\left(Y(k), \mathbb{R}^{1, d-1}\right)$.

$A^{\left\{i_{1}\right.} B^{\left.i_{2}\right\} i_{3}} \theta^{\left.i_{3} i_{2}\right\}}=A^{i_{1}} B^{i_{2} i_{3}} \theta^{i_{3} i_{2}}+A^{i_{2}} B^{i_{1} i_{3}} \theta^{i_{3} i_{1}}$; these indices are raised and lowered by means of the Euclidian metric tensors $\delta^{i j}, \delta_{i j}, \delta_{j}^{i}$. Second, the products $B_{i_{1} j_{1}}^{i_{2} j_{2}}, A^{i_{2} j_{2}, i_{1} j_{1}}, F^{i_{1} j_{1}, i}, L^{i_{2} j_{2}, i_{1} j_{1}}$ are determined by explicit relations (see, Ref. [31] for details),

$$
\begin{aligned}
B^{i_{2} j_{2}}{ }_{i_{1} j_{1}}= & \left(g_{0}^{i_{2}}-g_{0}^{j_{2}}\right) \delta_{i_{1}}^{i_{2}} \delta_{j_{1}}^{j_{2}}+\left(t_{j_{1}}{ }^{j_{2}} \theta^{j_{2} j_{1}}+t_{j_{1}}^{j_{2}+} \theta^{j_{1} j_{2}}\right) \delta_{i_{1}}^{i_{2}}-\left(t_{i_{1}}^{+i_{2}} \theta^{i_{2} i_{1}}+t^{i_{2}}{ }_{i_{1}} \theta^{i_{1} i_{2}}\right) \delta_{j_{1}}^{j_{2}}, \\
A^{i_{2} j_{2}, i_{1} j_{1}}= & t^{i_{1} j_{2}} \delta^{i_{2} j_{1}}-t^{i_{2} j_{1}} \delta^{i_{1} j_{2}}, \\
F^{i_{2} j_{2}, i}= & t^{i_{2} j_{2}}\left(\delta^{j_{2} i}-\delta^{i_{2} i}\right), \\
L^{i_{2} j_{2}, i_{1} j_{1}}= & \frac{1}{4}\left\{\delta^{i_{2} i_{1}} \delta^{j_{2} j_{1}}\left[2 g_{0}^{i_{2}} \delta^{i_{2} j_{2}}+g_{0}^{i_{2}}+g_{0}^{j_{2}}\right]-\delta^{j_{2}\left\{i_{1}\right.}\left[t^{\left.j_{1}\right\} i_{2}} \theta^{\left.i_{2} j_{1}\right\}}+t^{\left.i_{2} j_{1}\right\}+} \theta^{\left.j_{1}\right\} i_{2}}\right]\right. \\
& \left.-\delta^{i_{2}\left\{i_{1}\right.}\left[t^{\left.j_{1}\right\} j_{2}} \theta^{\left.j_{2} j_{1}\right\}}+t^{\left.j_{2} j_{1}\right\}+} \theta^{\left.j_{1}\right\} j_{2}}\right]\right\} .
\end{aligned}
$$

Third, the bosonic operator-valued quantities $C_{i_{2}}{ }^{i_{1}}$ in Table 2 have the following definition and Hermitian conjugation properties:

$$
C_{i_{2}}{ }^{i_{1}}=2\left(-g_{0}^{i_{1}} \delta_{i_{2}}{ }^{i_{1}}+t^{i_{1}}{ }_{i_{2}} \theta^{i_{2} i_{1}}+t_{i_{2}}{ }^{i_{1}+} \theta^{i_{1} i_{2}}\right), \quad\left(C_{i_{2}}{ }^{i_{1}}\right)^{+}=C_{i_{2}}{ }^{i_{1}} .
$$

For completeness, we list below the obvious additional properties of antisymmetry and Hermitian conjugation for the operators in Eqs.(2.22)-(2.25),

$$
\begin{aligned}
& A^{i_{2} j_{2}, i_{1} j_{1}}=-A^{i_{1} j_{1}, i_{2} j_{2}} \quad A_{i_{1} j_{1}, i_{2} j_{2}}^{+}=\left(A_{i_{1} j_{1}, i_{2} j_{2}}\right)^{+}=t_{i_{2} j_{1}}^{+} \delta^{j_{2} i_{1}}-t_{i_{1} j_{2}}^{+} \delta^{i_{2} j_{1}},
\end{aligned}
$$

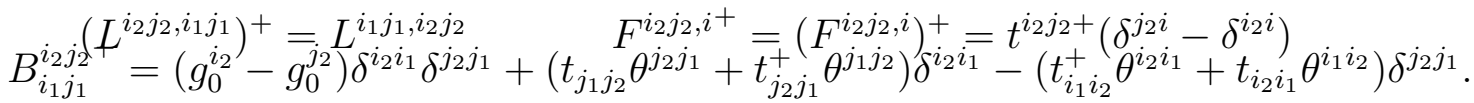

We call the algebra of operators $o_{I}(2.18)$ a half-integer higher-spin symmetry algebra in Minkowski space with a Young tableaux having $k$ rows 6 (or, simply, half-integer HS symmetry superalgebra in Minkowski space) and denote it as $\mathcal{A}^{f}\left(Y(k), \mathbb{R}^{1, d-1}\right)$.

\footnotetext{
${ }^{6}$ As in the case of bosonic fields [31, one should not identify the term "higher-spin symmetry superalgebra" used here for a free HS formulation starting from the paper [55] with the algebraic structure known as "higher-spin superalgebra" (see, for instance, Ref.[10]) used to describe HS interactions.
}

\begin{tabular}{||c||c|c|c||}
\hline \hline$[\downarrow, \rightarrow]$ & $t_{0}$ & $t_{i_{1}}$ & $t^{i_{1}+}$ \\
\hline \hline$t_{0}$ & $-2 l_{0}$ & $2 l_{i_{1}}$ & $2 l^{i_{1}+}$ \\
\hline$t_{i_{2}}$ & $2 l_{i_{2}}$ & $4 l_{i_{2} i_{1}}$ & $C_{i_{2}{ }^{{ }_{1}}}$ \\
\hline$t^{i_{2}+}$ & $2 l^{i_{2}+}$ & $\left(C_{i_{1}}{ }^{{ }_{2}}\right)^{+}$ & $4 l^{i_{2} i_{1}+}$ \\
\hline \hline
\end{tabular}

Table 2: odd-odd part of HS symmetry superalgebra $\mathcal{A}^{f}\left(Y(k), \mathbb{R}^{1, d-1}\right)$. 
From Table 1, it is obvious that the D'Alembertian $l_{0}$, being a Casimir element of the Poincare algebra $i s o(1, d-1)$, belongs to the center of the superalgebra $\mathcal{A}^{f}\left(Y(k), \mathcal{R}^{1, d-1}\right)$ as well.

Now, we are able to describe shortly the structure of the Lorentz-module $P_{k}^{d}\left(a^{+}\right)$of all finite string-like Dirac vectors of the form given by the Eq. (2.6) (see footnote 5) on a base of generalization of Howe duality [61] on a case of half-integer spin representations of Lorentz group $S O(1, d-1)$. The Howe dual superalgebra to $s o(1, d-1)$ is $\operatorname{osp}(1 \mid 2 k)$ if $k=\left[\frac{d-1}{2}\right]$ with the following basis elements [61] for arbitrary $i, j=1, \ldots, k$,

$$
\hat{t}_{i}=\tilde{\gamma}^{\mu} a_{i \mu}^{+}, \quad \hat{t}^{i}=\tilde{\gamma}^{\mu} a_{\mu}^{i}, \quad \hat{l}_{i j}=a_{i \mu}^{+} a_{j}^{\mu+}, \quad \hat{t}_{i}^{j}=\frac{1}{2}\left\{a_{i \mu}^{+}, a^{j \mu}\right\}, \quad \hat{l}^{i j}=a_{\mu}^{i} a^{j \mu},
$$

which is distinguished from the elements of $\mathcal{A}^{f}\left(Y(k), \mathcal{R}^{1, d-1}\right)$ by the sign "hat". Their nonvanishing supercommutator's relations have the form

$$
\begin{array}{ll}
\left\{\hat{t}_{i}, \hat{t}_{j}\right\}=2 \hat{l}_{i j}, & \left\{\hat{t}^{i}, \hat{t}^{j}\right\}=2 \hat{l}^{i j}, \\
{\left[\hat{t}_{i}, \hat{t}^{j}\right\}=2 \hat{t}_{i}^{j},} & {\left[\hat{t}^{i}, \hat{t}_{i_{1}}{ }^{j_{1}}\right]=-\hat{t}^{j_{1}} \delta_{i_{1}}^{i},} \\
{\left[\hat{t}_{i}, \hat{t}_{i_{1}}{ }^{j_{1}}\right]=\hat{t}_{i_{1}} \delta_{i}^{j_{1}},} & {\left[\hat{t}^{i}, \hat{l}_{i_{1} j_{1}}\right]=-\hat{t}_{\left\{i_{1}\right.} \delta_{\left.j_{1}\right\}}^{i},} \\
{\left[\hat{t}_{i}, \hat{l}^{i_{1} j_{1}}\right]=\hat{t}^{\left\{i_{1}\right.} \delta_{i}^{\left.j_{1}\right\}},} & \\
{\left[\hat{t}_{i_{1}}{ }^{j_{1}}, \hat{t}_{i_{2}}{ }^{j_{2}}\right]=\hat{t}_{i_{1}}{ }^{j_{2}} \delta_{i_{2}}^{j_{1}}-\hat{t}_{i_{2}}{ }^{j_{1}} \delta_{i_{1}}^{j_{2}},} & {\left[\hat{l}^{i_{2} j_{2}}, \hat{l}_{i_{1} j_{1}}\right]=\delta_{\left\{i_{1}\right.}^{\left\{i_{2}\right.} \hat{t}_{\left.j_{1}\right\}} j^{\left.j_{2}\right\}},} \\
{\left[\hat{t}_{i_{1}}{ }^{j_{1}}, \hat{l}_{i_{2} j_{2}}\right]=\hat{l}_{i_{1}\left\{j_{2}\right.} \delta_{\left.i_{2}\right\}}^{j_{1}},} & {\left[\hat{t}_{i_{1}}{ }^{j_{1}}, \hat{l}^{i_{2} j_{2}}\right]=-\hat{l}^{j_{1}\left\{j_{2}\right.} \delta_{i_{1}}^{\left.i_{2}\right\}} .}
\end{array}
$$

The elements $t^{i}, t^{i+}, l^{i j}, l^{i j+}, t^{i_{1} j_{1}}, t_{i_{1} j_{1}}^{+}, g_{0}^{i}$ from HS symmetry superalgebra $\mathcal{A}^{f}\left(Y(k), \mathbb{R}^{1, d-1}\right)$ are derived from the basis elements of $\operatorname{ssp}(1 \mid 2 k)$ by the rules (for $s p(2 k)$ case see Ref. [31]),

$$
t^{i}=\hat{t}^{i}, \quad t^{i+}=\hat{t}_{i} ; \quad l_{i j}^{+}=\frac{1}{2} \hat{l}_{i j}, \quad l^{i j}=\frac{1}{2} \hat{l}^{i j}, \quad t_{i}^{j}=\hat{t}_{i}^{j} \theta^{j i}, \quad t^{j}{ }_{i}^{+}=\hat{t}_{i}^{j} \theta^{i j}, \quad g_{0}^{i}=-\hat{t}_{i}{ }^{i} .
$$

The rest elements $\left\{l^{i}, l^{i+}, t_{0}, l_{0}\right\}$ of the superalgebra $\mathcal{A}^{f}\left(Y(k), \mathbb{R}^{1, d-1}\right)$ forms the subsuperalgebra which describes the isometries of Minkowski space $\mathbb{R}^{1, d-1}$. It may be realized as direct sum of $k$-dimensional commutative algebra $T^{k}=\left\{l_{i}\right\}$ and its dual $T^{k *}=\left\{l^{i+}\right\}$,

$$
\left\{l^{i}, l^{i+}, t_{0}, l_{0}\right\}=\left(T^{k} \oplus T^{k *} \oplus\left[T^{k}, T^{k *}\right]\right), \quad\left[T^{k}, T^{k *}\right] \sim l_{0}=-t_{0}^{2},
$$

so that half-integer HS symmetry algebra $\mathcal{A}^{f}\left(Y(k), \mathbb{R}^{1, d-1}\right)$ represents the semidirect sum of the orthosymplectic superalgebra $\operatorname{ssp}(1 \mid 2 k)$ [as an algebra of internal derivations of $\left(T^{k} \oplus T^{k *}\right)$ ] with $\left.\left(T^{k} \oplus T^{k *} \oplus\left[T^{k}, T^{k *}\right]\right)\right]$

$$
\mathcal{A}^{f}\left(Y(k), \mathbb{R}^{1, d-1}\right)=\left(T^{k} \oplus T^{k *} \oplus\left[T^{k}, T^{k *}\right]\right) \boxplus o s p(1 \mid 2 k) .
$$

Note, the elements $g_{0}^{i}$, form a basis in the Cartan subalgebra whereas $t^{i}, l^{i j}, t_{i}^{j}$ are the basis of low-triangular subsuperalgebra in $\operatorname{osp}(1 \mid 2 k)$.

Having the identification (2.35), we are able to conclude that half-integer-spin finite-dimensional irreducible representations of the Lorentz algebra $s o(1, d-1)$, subject to the Young tableaux $Y T(k)$, and realized on spin-tensor fields (2.1), are equivalently extracted by the annihilation of all elements from the $s o(1, d-1)$-module $P_{k}^{d}\left(a^{+}\right)$by the low-triangular subalgebra of $o s p(1 \mid 2 k)$,

${ }^{7}$ The construction of algebra $\mathcal{A}^{f}\left(Y(k), \mathbb{R}^{1}, d-1\right)$ in the Eq. (2.35) is similar to the realization of the Poincare algebra $i s o(1, d-1)$ by means of Lorentz algebra and Abelian subalgebra $T(1, d-1)$ of space-time translations which looks as follows, iso $(1, d-1)=T(1, d-1) \boxplus s o(1, d-1)$. 
along with the weight conditions, given by Eqs. (2.10), with respect to its Cartan subalgebra, which has the form, in terms of independent relations,

$$
t^{i}|\Psi\rangle=0, \quad t_{i}^{j}|\Psi\rangle=0, \quad \hat{t}_{i}^{i}|\Psi\rangle \equiv-g_{0}^{i}|\Psi\rangle=-\left(s_{i}+\frac{d}{2}\right)|\Psi\rangle
$$

Half-integer spin finite-dimensional irreducible representations of the Poincare algebra $i s o(1, d-$ 1) can be easily obtained from those for the Lorentz algebra, by adding the only independent condition, given by the Dirac operator:

$$
t_{0}|\Psi\rangle=0
$$

which lifts the set $P_{k}^{d}\left(a^{+}\right)$to the Poincare module (for another realization of the bosonic Poincare module from the Lorentz module, see Ref. [8]).

The derivation of the HS symmetry algebra has not yet provided a construction of the BRST operator $Q$ with respect to the elements $o_{I}$ from $\mathcal{A}^{f}\left(Y(k), \mathcal{R}^{1, d-1}\right)$, due to the presence of nondegenerate (in the Fock space $\mathcal{H}$ ) operators $g_{0}^{i}$, determining, according to Eqs. (2.18), the system of $o_{I}$ as that with a second-class constraint system. Because of the general property [36, 38] of the BFV-method, such a BRST operator $Q$ would not reproduce a correct set of initial constraints (2.9), (2.16) in the zero ghost $Q$-cohomology subspace of the total Hilbert space, $\mathcal{H}_{\text {tot }}\left(\mathcal{H} \subset \mathcal{H}_{\text {tot }}\right)$. To solve this problem, we consider a procedure of converting the set of $o_{I}$ into that of $O_{I}$, which would be of first-class constraints only, in subspaces controlled by extended particle-number operators $G_{0}^{I}$.

\section{Converted HS Symmetry Superalgebras for YT with $k$ Rows}

To convert the set of $o_{I}$ operators, we shall present the construction of an auxiliary representation for the orthosymplectic superalgebra $\operatorname{osp}(1 \mid 2 k)$ with second-class constraints only, in terms of oscillator operators in an auxiliary Fock space over an appropriate Heisenberg-Weyl superalgebra, and then extend the latter to the case of massive half-integer HS fields subject to the same Young tableaux $Y\left(s_{1}, \ldots, s_{k}\right)$.

\subsection{Auxiliary Representation for $\operatorname{osp}(1 \mid 2 k)$ Superalgebra}

Since the $\operatorname{osp}(1 \mid 2 k)$ generators alone are the second-class constraints in $\mathcal{A}^{f}\left(Y(k), \mathbb{R}^{1, d-1}\right)$, which are to be converted, then, instead of the additional parts $o_{I}^{\prime}$ in the representation of converted constraints $O_{I}=o_{I}+o_{I}^{\prime}$ within an additive conversion procedure of the BRST approach (see, for details, e.g., [47], [59], [64], and for the general concept, [62]), it is sufficient to use only some of them, namely $\left\{o_{a}^{\prime}, o_{a}^{\prime+}\right\}$. These additional parts $o_{I}^{\prime}$ act in a new Fock space $\mathcal{H}^{\prime}$, subject to the standard relation $\mathcal{H}^{\prime} \cap \mathcal{H}=\emptyset$. Algebraic structures of the sets $o_{I}^{\prime}$ and $O_{I}$ are determined by the requirement of supercommutativity, $\left[o_{I}, o_{J}^{\prime}\right\}=0$. This provides the fact that these sets have the same multiplication laws as those for the $\operatorname{osp}(1 \mid 2 k)$ superalgebra and for the superalgebra $\mathcal{A}_{c}^{f}\left(Y(k), \mathbb{R}^{1, d-1}\right)$, respectively, for $o_{I}^{\prime}$ and $O_{I}$.

Therefore, one has to obtain a new operator realization for the $\operatorname{ssp}(1 \mid 2 k)$ algebra $o_{I}^{\prime}$. An effective solution of this problem can be provided by a special procedure, known in the mathematical literature as the generalized Verma module construction [70, 71], applied to the latter superalgebra. This procedure is presented explicitly in Appendix A. 


\subsection{Scalar Oscillator Realization of Additional Parts to Constraints}

Prior to an explicit oscillator realization for the additional parts $o_{I}^{\prime}$, it should be noted that in the case of the superalgebra of half-integer HS mixed-symmetric fields $\mathcal{A}^{f}\left(Y(2), \mathbb{R}^{1, d-1}\right)$ an auxiliary representation of its converted subsuperalgebra $\operatorname{ssp}(1 \mid 4)$ of second-class constraints was constructed in [59]. For a more general case of fermionic HS fields characterized by a Young tableaux with $k \geq 2$ rows in a symmetric basis, we enlarge the results of [72 from the case of a Lie algebra to case of the orthosymplectic superalgebra $\operatorname{osp}(1 \mid 2 k)$, so as to transform the generalized Verma module (a special representation, whose detailed construction for $\operatorname{ssp}(1 \mid 2 k)$ is described in Appendix A) to an oscillator form, being suitable to obtain the BRST operator. As a result, we present here the oscillator representation (found on a basis of calculations made in Appendix 3.1) for the operators $o_{I}^{\prime}$, first, for the operators with the Hermitian conjugation sign, $"+", t_{i}^{\prime+}, l_{i j}^{\prime+}, t_{r s}^{\prime+}$,

$$
\begin{aligned}
& t_{i}^{\prime+}=f_{i}^{+}+2 b_{i i}^{+} f_{i}+4 \sum_{l=1}^{i-1} b_{l i}^{+} f_{l}, \quad l_{i j}^{\prime+}=b_{i j}^{+}, \\
& t_{r s}^{\prime+}=d_{r s}^{+}-\sum_{n=1}^{r-1} d_{n r} d_{n s}^{+}-\sum_{n=1}^{k}\left(1+\delta_{n r}\right) b_{n s}^{+} b_{r n}-\left[4 \sum_{n=r+1}^{s-1} b_{n s}^{+} f_{n}+\left(f_{s}^{+}+2 b_{s s}^{+} f_{s}\right)\right] f_{r},
\end{aligned}
$$

second, for the particle number operators, $g_{0}^{\prime i}$,

$$
g_{0}^{\prime i}=f_{i}^{+} f_{i}+\sum_{l \leq m} b_{l m}^{+} b_{l m}\left(\delta^{i l}+\delta^{i m}\right)+\sum_{r<s} d_{r s}^{+} d_{r s}\left(\delta^{i s}-\delta^{i r}\right)+h^{i} .
$$

The quantities $h^{i}, i=1, \ldots, k$ in (3.3) and below are arbitrary dimensionless constants, introduced in Appendix $\mathrm{A}$, whose specific values are determined in Section 5 and based on a solution of a special spectral problem.

Third, for the Grassmann-odd "gamma-traceless" elements $t_{i}^{\prime}$ we have,

$$
\begin{aligned}
t_{i}^{\prime}= & -2 \sum_{n=1}^{i-1}\left\{\sum_{m=1}^{n-1} d_{m n}^{+} d_{m i}-\sum_{p=0}^{i-n-1} \sum_{k_{1}=n+1}^{i-1} \ldots \sum_{k_{p}=n+p}^{i-1} C^{k_{p} i}\left(d^{+}, d\right) \prod_{j=1}^{p} d_{k_{j-1} k_{j}}\right. \\
+ & \left.\sum_{m=1}^{k}\left(1+\delta_{m i}\right) b_{m n}^{+} b_{m i}-\left[4 \sum_{m=n+1}^{i-1} b_{n m}^{+} f_{m}-f_{n}^{+}\right] f_{i}\right\} f_{n} \\
+ & 2 \sum_{n=i+1}^{k}\left\{d_{i n}^{+}-\sum_{m=1}^{i-1} d_{m n}^{+} d_{m i}-\sum_{m=1}^{k}\left(1+\delta_{m i}\right) b_{n m}^{+} b_{i m}\right\} f_{n} \\
& -2\left(\sum_{l=1}^{k}\left(1+\delta_{i l}\right) b_{i l}^{+} b_{i l}-\sum_{s>i} d_{i s}^{+} d_{i s}+\sum_{r<i} d_{r i}^{+} d_{r i}+h^{i}\right) f_{i} \\
& +\sum_{n=1}^{k}\left(1+\delta_{n i}\right)\left\{2 \sum_{m=n+1}^{k} b_{n m}^{+} f_{m}-\frac{1}{2}\left(f_{n}^{+}-2 b_{n n}^{+} f_{n}\right)\right\} b_{n i} .
\end{aligned}
$$

Next, for the "traceless" elements $l_{l m}^{\prime}$, separately for $l=m$ and for $l<m$, corresponding to the secondary constraints, we obtain

$$
\begin{aligned}
l_{l l}^{\prime}= & -\left[2 \sum_{n=l+1}^{k}\left\{d_{l n}^{+}-\sum_{n^{\prime}=1}^{l-1} d_{n^{\prime} n}^{+} d_{n^{\prime} l}-\sum_{n^{\prime}=1}^{k}\left(1+\delta_{n^{\prime} l}\right) b_{n^{\prime} n}^{+} b_{n^{\prime} l}\right\} f_{n}\right. \\
& \left.-\sum_{n=1}^{k}\left(1+\delta_{n l}\right)\left\{-2 \sum_{m=n+1}^{k} b_{n m}^{+} f_{m}+\frac{1}{2}\left[f_{n}^{+}-\left(1-\delta_{n l}\right) 2 b_{n n}^{+} f_{n}\right]\right\} b_{l n}\right] f_{l}+l_{l l}^{l b},
\end{aligned}
$$




$$
\begin{aligned}
l_{l m}^{\prime}= & -\left[\sum _ { n = l + 1 } ^ { m - 1 } \left\{-\sum_{n^{\prime}=1}^{n-1} d_{n^{\prime} n}^{+} d_{n^{\prime} m}+\sum_{p=0}^{m-n-1} \sum_{k_{1}=n+1}^{m-1} \ldots \sum_{k_{p}=n+p}^{m-1} C^{k_{p} m}\left(d^{+}, d\right) \prod_{j=1}^{p} d_{k_{j-1} k_{j}}\right.\right. \\
& \left.-\sum_{n^{\prime}=1}^{k}\left(1+\delta_{n^{\prime} m}\right) b_{n^{\prime} n}^{+} b_{n^{\prime} m}+\left[4 \sum_{n^{\prime}=n+1}^{m-1} b_{n^{\prime} n}^{+} f_{n^{\prime}}-f_{n}^{+}\right] f_{m}\right\} f_{n} \\
& +\sum_{n=m+1}^{k}\left\{d_{m n}^{+}-\sum_{n^{\prime}=1}^{m-1} d_{n^{\prime} n}^{+} d_{n^{\prime} m}-\sum_{n^{\prime}=1}^{k}\left(1+\delta_{n^{\prime} m}\right) b_{n^{\prime} n}^{+} b_{m n^{\prime}}\right\} f_{n} \\
& -\left(\sum_{n=1}^{k}\left(1+\delta_{n m}\right) b_{m n}^{+} b_{m n}-\sum_{s>m} d_{m s}^{+} d_{m s}+\sum_{r<m} d_{r m}^{+} d_{r m}+h^{m}\right) f_{m} \\
& \left.+\frac{1}{2} \sum_{n=1}^{k}\left(1+\delta_{n m}\right)\left\{2 \sum_{n^{\prime}=n+1}^{k} b_{n n^{\prime}}^{+} f_{n^{\prime}}-\frac{1}{2}\left[f_{n}^{+}-\left(1-\delta_{n l}\right) 2 b_{n n}^{+} f_{n}\right]\right\} b_{n m}\right] f_{l} \\
& -\left[\sum_{n=m+1}^{k}\left\{d_{l n}^{+}-\sum_{n^{\prime}=1}^{l-1} d_{n^{\prime} n}^{+} d_{n^{\prime} l}-\sum_{n^{\prime}=1}^{k}\left(1+\delta_{n^{\prime} l}\right) b_{n n^{\prime}}^{+} b_{l n^{\prime}}\right\} f_{n}\right. \\
& \left.+\frac{1}{2} \sum_{n=1}^{k}\left(1+\delta_{n l}\right)\left\{2 \sum_{n^{\prime}=n+1}^{k} b_{n n^{\prime}}^{+} f_{n^{\prime}}-\frac{1}{2}\left[f_{n}^{+}-\left(1-\delta_{n m}\right) 2 b_{n n}^{+} f_{n}\right]\right\} b_{n l}\right] f_{m}+l_{l m}^{\prime b},
\end{aligned}
$$

with the use of the $\left(f_{l}, f_{l}^{+}\right)$-independent bosonic operators $l_{l m}^{\prime b}$, first obtained for the symplectic $s p(2 k)$ algebra in [31], for $l=m$, and, for $l>m$, respectively,

$$
\begin{aligned}
l_{l l}^{\prime b}= & \frac{1}{4} \sum_{n=1, n \neq l}^{k} b_{n n}^{+} b_{l n}^{2}+\frac{1}{2} \sum_{n=1}^{l-1}\left[\sum_{n^{\prime}=1}^{n-1} d_{n^{\prime} n}^{+} d_{n^{\prime} l}+\sum_{n^{\prime}=n+1}^{k}\left(1+\delta_{n^{\prime} l}\right) b_{n n^{\prime}}^{+} b_{n^{\prime} l}\right. \\
& \left.-\sum_{p=0}^{l-n-1} \sum_{k_{1}=n+1}^{l-1} \ldots \sum_{k_{p}=n+p}^{l-1} C^{k_{p} l}\left(d^{+}, d\right) \prod_{j=1}^{p} d_{k_{j-1} k_{j}}\right] b_{n l} \\
& +\left(\sum_{n=l}^{k} b_{n l}-\sum_{s>l} d_{l s}^{+} d_{l s}+\sum_{r<l} d_{r l}^{+} d_{r l}+h^{l}\right) b_{l l} \\
& -\frac{1}{2} \sum_{n=l+1}^{k}\left[d_{l n}^{+}-\sum_{n^{\prime}=1}^{l-1} d_{n^{\prime} n}^{+} d_{n^{\prime} l}-\sum_{n^{\prime}=n+1}^{k}\left(1+\delta_{n^{\prime} l}\right) b_{n^{\prime} n}^{+} b_{n^{\prime} l}\right] b_{l n} \\
l_{l m}^{\prime b} & -\frac{1}{4} \sum_{n=1}^{m-1}\left(1+\delta_{n l}\right)\left[-\sum_{n^{\prime}=1}^{n-1} d_{n^{\prime} n}^{+} d_{n^{\prime} m}-\sum_{n^{\prime}=n}^{k}\left(1+\delta_{n^{\prime} m}\right) b_{n^{\prime} n}^{+} b_{n^{\prime} m}\right. \\
& \left.+\sum_{p=0}^{m-n-1} \sum_{k_{1}=n+1}^{m-1} \ldots \sum_{k_{p}=n+p}^{m-1} C^{k_{p} n}\left(d^{+}, d\right) \prod_{j=1}^{m} d_{k_{j-1} k_{j}}\right] b_{n l} \\
& -\frac{1}{4} \sum_{n=m+1}^{k}\left[d_{m n}^{+}-\sum_{n^{\prime}=1}^{m-1} d_{n^{\prime} n}^{+} d_{n^{\prime} m}-\sum_{n^{\prime}=l+1}^{k}\left(1+\delta_{n^{\prime} m}\right) b_{n^{\prime} n}^{+} b_{m n^{\prime}}\right] b_{l n} \\
& +\frac{1}{4}\left(\sum_{n=m}^{k} b_{l n}^{+} b_{l n}+\sum_{n=l+1}^{k}\left(1+\delta_{n m}\right) b_{n m}^{+} b_{n m}-\sum_{s>l} d_{l s} d_{l s}-\sum_{s>m} d_{m s}^{+} d_{m s}\right. \\
& \left.\sum_{r<l} d_{r l}^{+} d_{r l}+\sum_{r<m} d_{r m}^{+} d_{r m}+h^{l^{\prime}}+h^{m^{\prime}}\right) b_{l m} \\
& \\
& \\
&
\end{aligned}
$$




$$
\begin{aligned}
& -\frac{1}{4} \sum_{n=1}^{l-1}\left[-\sum_{n^{\prime}=1}^{n-1} d_{n^{\prime} n}^{+} d_{n^{\prime} l}+\sum_{p=0}^{l-n-1} \sum_{k_{1}=n+1}^{l-1} \ldots \sum_{k_{p}=n+p}^{l-1} C^{k_{p} n}\left(d^{+}, d\right) \prod_{j=1}^{p} d_{k_{j-1} k_{j}}\right. \\
& \left.-\sum_{n^{\prime}=n+1}^{k}\left(1+\delta_{n^{\prime} l}\right) b_{n^{\prime} n}^{+} b_{n^{\prime} l}\right] b_{n m}-\frac{1}{4} \sum_{n=l+1}^{k}\left(1+\delta_{n m}\right)\left[d_{l n}^{+}-\sum_{n^{\prime}=1}^{l-1} d_{n^{\prime} n}^{+} d_{n^{\prime} l}\right] b_{m n} .
\end{aligned}
$$

In turn, for the "mixed symmetry" elements $t_{r s}^{\prime}$, we have the representation

$$
\begin{aligned}
t_{r s}^{\prime}= & -\sum_{n=1}^{r-1} d_{n r}^{+} d_{n s}+\sum_{p=0}^{s-r-1} \sum_{k_{1}=r+1}^{s-1} \ldots \sum_{k_{p}=r+p}^{s-1} C^{k_{p} s}\left(d^{+}, d\right) \prod_{j=1}^{p} d_{k_{j-1} k_{j}} \\
& -\sum_{n=1}^{k}\left(1+\delta_{n s}\right) b_{n r}^{+} b_{n s}+\left[4 \sum_{n=r+1}^{s-1} b_{r n}^{+} f_{n}+\left(2 b_{r r}^{+} f_{r}-f_{r}^{+}\right)\right] f_{s}, \quad k_{0} \equiv r
\end{aligned}
$$

where the operators $C^{r s}\left(d, d^{+}\right)$in Eqs. (3.4), (3.6)-(3.9) were first derived in [31] for the symplectic $s p(2 k)$ algebra, and are determined, for $r<m$, as follows:

$$
\begin{aligned}
C^{r s}\left(d^{+}, d\right) \equiv & \left(h^{r}-h^{s}-\sum_{n=s+1}^{k}\left(d_{r n}^{+} d_{r n}+d_{s n}^{+} d_{s n}\right)+\sum_{n=r+1}^{s-1} d_{n s}^{+} d_{n s}-d_{r s}^{+} d_{r s}\right) d_{r s} \\
& +\sum_{n=s+1}^{k}\left\{d_{s n}^{+}-\sum_{n^{\prime}=1}^{s-1} d_{n^{\prime} n}^{+} d_{n^{\prime} s}\right\} d_{r n} . .
\end{aligned}
$$

To construct the additional parts $o_{I}^{\prime}$ in (3.1)-(3.9), we have introduced a new Fock superspace $\mathcal{H}^{\prime}$, generated by $2 k$ fermionic, $f_{i}^{+}, f_{i}$, and $2 k^{2}$ bosonic, $b_{i j}^{+}, d_{r s}^{+}, b_{i j}, d_{r s}, i, j, r, s=1, \ldots, k ; i \leq j ; r<$ $s$, creation and annihilation operators, whose numbers are equal to those of the second-class constraints $o_{a}^{\prime}, o_{a}^{\prime+}$, with the standard (only nonvanishing) commutation relations

$$
\left\{f_{i}, f_{j}^{+}\right\}=\delta_{i j},, \quad\left[b_{i_{1} j_{1}}, b_{i_{2} j_{2}}^{+}\right]=\delta_{i_{1} i_{2}} \delta_{j_{1} j_{2}}, \quad\left[d_{r_{1} s_{1}}, d_{r_{2} s_{2}}^{+}\right]=\delta_{r_{1} r_{2}} \delta_{s_{1} s_{2}} .
$$

As usual, the additional parts $o_{a}^{\prime}\left(B, B^{+}\right), o_{a}^{\prime+}\left(B, B^{+}\right)$, regarded as polynomials in the oscillator variables $\left(B, B^{+}\right) \equiv\left(f_{i}, b_{i j}, d_{r s} ; f_{i}^{+}, b_{i j}^{+}, d_{r s}^{+}\right)$, do not obey the standard properties

$$
\left(t_{i}^{\prime}\right)^{+} \neq t_{i}^{\prime+}, \quad\left(l_{i j}^{\prime}\right)^{+} \neq l_{i j}^{\prime+}, i \leq j, \quad\left(t_{r s}^{\prime}\right)^{+} \neq t_{r s}^{\prime+}, r<s
$$

if one should use the usual rules of Hermitian conjugation for the new creation and annihilation operators,

$$
\left(f_{i}\right)^{+}=f_{i}^{+}, \quad\left(b_{i j}\right)^{+}=b_{i j}^{+}, \quad\left(d_{r s}\right)^{+}=d_{r s}^{+},
$$

with respect to the same definition of the odd scalar product (2.15), however, residing in $\mathcal{H}^{\prime}$. To restore the proper Hermitian conjugation properties for the additional parts, we define another odd scalar product in the Fock space $\mathcal{H}^{\prime}$, using the following relations:

$$
\left\langle\tilde{\Psi}_{1} \mid \Psi_{2}\right\rangle_{\mathrm{new}}=\left\langle\tilde{\Psi}_{1}\left|K^{\prime}\right| \Psi_{2}\right\rangle
$$

for any vectors $\left|\Psi_{n}\right\rangle$ (Dirac spinors), $n=1,2$, with a bosonic operator $K^{\prime}$, being nondegenerate in $\mathcal{H}^{\prime}$ but yet unknown otherwise. The operator is to be determined as a solution of the equations

$$
\left\langle\tilde{\Psi}_{1}\left|K^{\prime} E^{-\prime \alpha}\right| \Psi_{2}\right\rangle=\left\langle\tilde{\Psi}_{2}\left|K^{\prime} E^{\prime \alpha}\right| \Psi_{1}\right\rangle^{*}, \quad\left\langle\tilde{\Psi}_{1}\left|K^{\prime} g_{0}^{\prime i}\right| \Psi_{2}\right\rangle=\left\langle\tilde{\Psi}_{2}\left|K^{\prime} g_{0}^{\prime i}\right| \Psi_{1}\right\rangle^{*} .
$$


for all $\left(E^{-\alpha \alpha}, E^{\prime \alpha}\right)=\left(t_{i}^{\prime+}, l_{i j}^{\prime+}, t_{r s}^{\prime+} ; t_{i}^{\prime}, l_{i j}^{\prime}, t_{r s}^{\prime}\right)$. A solution of Eqs.(3.15) exists in a form being Hermitian with respect to the standard odd scalar product in $\mathcal{H}^{\prime},\langle\mid\rangle$, similar to (2.15) in $\mathcal{H}$,

$$
\begin{array}{r}
K^{\prime}=Z^{+} Z, \quad Z=\sum_{\left.\vec{n}_{l}^{0}=\overrightarrow{0}_{l}^{0} \vec{n}_{i j}, \vec{p}_{r s}\right)=(\overrightarrow{0}, \overrightarrow{0})}^{\overrightarrow{1}_{l}^{0}}\left|\vec{N}^{f}\right\rangle_{V} \frac{1}{\left(\vec{n}_{i j}\right) !\left(\vec{p}_{r s}\right) !}\langle 0| \prod_{r, s>r}^{k} d_{r s}^{p_{r s}} \prod_{i, j \geq i}^{k} b_{i j}^{n_{i j}} \prod_{l=1}^{k} f_{k-l+1}^{n_{k-l+1}^{0},},(3.16) \\
Z^{+}=\sum_{\left.\vec{n}_{l}^{\prime 0}=\overrightarrow{0}_{l}^{0} \vec{n}_{i j}^{\prime}, \vec{p}_{r s}^{\prime}\right)=(\overrightarrow{0}, \overrightarrow{0})}^{\infty} \frac{1}{\left(\vec{n}_{i j}\right) !\left(\vec{p}_{r s}\right) !} \prod_{l=1}^{k}\left(f_{l}^{+}\right)^{n_{l}^{\prime 0}} \prod_{i, j \geq i}^{k}\left(b_{i j}^{+}\right)^{n_{i j}^{\prime}} \prod_{r, s>r}^{k}\left(d_{r s}^{+}\right)^{p_{r s}^{\prime}}|0\rangle_{V}\left\langle\vec{N}^{\prime f}\right|,
\end{array}
$$

where the symbols $\left(\vec{n}_{i j}\right)$ !, $\left(\vec{p}_{r s}\right)$ ! imply the products of factorials, $\left(\vec{n}_{i j}\right) !=\prod_{i, j \geq i}^{k} n_{i j}$ !, $\left(\vec{p}_{r s}\right)$ ! = $\prod_{r, s>r}^{k} p_{r s}$ !; the vector $\left|\vec{N}^{f}\right\rangle_{V}$ is determined in Appendix $\mathrm{A}$, and ${ }_{V}\left\langle\vec{N}^{f}\right|$ is a dual vector. A detailed calculation of the operator $K^{\prime}$ is described in Appendix B.

Let now turn to the case of massive fermionic HS fields whose system of second-class constraints contains, in addition to the elements of the $\operatorname{ssp}(1 \mid 2 k)$ superalgebra, the constraints of the isometry subalgebra of the Minkowski space, $t_{0}, l_{0}, l^{i}, l_{i}^{+}$.

\subsection{On Auxiliary Representations of Superalgebra $\mathcal{A}^{f}\left(Y(k), \mathbb{R}^{1, d-1}\right)$ for Massive HS Fields}

Analogous oscillator representations for the HS symmetry superalgebra of massive fermionic HS fields with mass $m$, where the massless Dirac equation, given by (2.2), is to be replaces by a massive equation, corresponding to the constraint $t_{0}\left(t_{0}=-\imath \tilde{\gamma}^{\mu} \partial_{\mu}+\tilde{\gamma} m\right)$, acting on the same string-vector (Dirac spinor) $|\Psi\rangle(\underline{2.6})$

$$
\left(\imath \gamma^{\mu} \partial_{\mu}-m\right) \Psi_{\left(\mu^{1}\right)_{n_{1}},\left(\mu^{2}\right)_{n_{2}}, \ldots,\left(\mu^{k}\right)_{n_{k}}}=0 \Longleftrightarrow\left(\imath \tilde{\gamma}^{\mu} \partial_{\mu}-\tilde{\gamma} m\right) \Psi_{\left(\mu^{1}\right)_{n_{1}},\left(\mu^{2}\right)_{n_{2}}, \ldots,\left(\mu^{k}\right)_{n_{k}}}=0
$$

can be constructed following the procedure described in Section 3.2 and realized in Appendices $\mathrm{A}$, B for the $\operatorname{osp}(1 \mid 2 k)$ superalgebra (see the remarks in Appendix A.1 for massive spintensors). In addition, because of the algebraic relation $\left(t_{0}\right)^{2}=-l_{0}$ from Table 2, we should also replace in this case the constraint $l_{0}$ by $l_{0}=\partial^{\mu} \partial_{\mu}+m^{2}$. Instead, following, in part, the research of the integer-spin case [31, we have used the procedure of dimensional reduction of the initial superalgebra $\mathcal{A}^{f}\left(Y(k), \mathbb{R}^{1, d}\right)$ for massless fermionic HS fields in a $(d+1)$-dimensional flat space-time to that of dimension $d, \mathbb{R}^{1, d-1}$.

To this end, we, first, write down the rules of dimensional reduction from the flat background $\mathbb{R}^{1, d}$ to $\mathbb{R}^{1, d-1}$,

$$
\begin{array}{lll}
\partial_{M}=\left(\partial_{\mu}, \imath m\right), & a_{i}^{M}=\left(a_{i}^{\mu}, b_{i}\right), & a_{i}^{M+}=\left(a_{i}^{\mu+}, b_{i}^{+}\right), \\
\tilde{\gamma}^{M}=\left(\tilde{\gamma}^{\mu}, \tilde{\gamma}\right), & M=(\mu, d), & \eta^{M N}=\operatorname{diag}(1,-1, \ldots,-1,-1),
\end{array}
$$

Second, we obtain, on a basis of the rules (3.19) for the set of the original elements $o_{I}$ from the massless HS symmetry superalgebra $\mathcal{A}^{f}\left(Y(k), \mathbb{R}^{1, d}\right)$, a representation for $\tilde{o}_{I}$ in the massive HS symmetry superalgebra $\mathcal{A}^{f}\left(Y(k), \mathbb{R}^{1, d-1}\right)$, as follows:

$$
\begin{array}{ll}
\tilde{t}_{0}=-\imath \tilde{\gamma}^{M} \partial_{M}=t_{0}+\tilde{\gamma} m, & \tilde{t}_{i}=\tilde{\gamma}^{M} a_{M}=t_{i}-\tilde{\gamma} b_{i}, \\
\tilde{l}_{0}=\partial^{M} \partial_{M}=l_{0}+m^{2}, & \tilde{t}_{i}^{+}=\tilde{\gamma}^{M} a_{M}^{+}=t_{i}^{+}-\tilde{\gamma} b_{i}^{+},
\end{array}
$$




$$
\begin{array}{ll}
\tilde{l}_{i}=-i a_{i}^{M} \partial_{M}=l_{i}+m b_{i}, & \tilde{l}_{i}^{+}=-i a_{i}^{+M} \partial_{M}=l_{i}^{+}+m b_{i}^{+}, \\
\tilde{l}_{i j}=\frac{1}{2} a_{i}^{M} a_{M j}=l_{i j}-\frac{1}{2} b_{i} b_{j}, & \tilde{l}_{i j}^{+}=\frac{1}{2} a_{i}^{M+} a_{M j}^{+}=l_{i j}^{+}-\frac{1}{2} b_{i}^{+} b_{j}^{+}, \\
\tilde{t}_{i j}=a_{i}^{M+} a_{M j} \theta^{j i}=t_{i j}^{+}-b_{i}^{+} b_{j} \theta^{j i}, & \tilde{t}_{i j}^{+}=a_{i}^{M} a_{M j}^{+} \theta^{j i}=t_{i j}^{+}-b_{i} b_{j}^{+} \theta^{j i}, \\
\tilde{g}_{0}^{i}=-a_{M i}^{+} a_{i}^{M}+\frac{d+1}{2}=g_{0}^{i}+b_{i}^{+} b_{i}+\frac{1}{2} . &
\end{array}
$$

The set of odd $\left(\tilde{t}_{0}, t_{i}^{+}, t_{i}\right)$ and even $\left.\left(\tilde{l}_{0}, l_{i}^{+}, l_{i}\right), l_{i j}, l_{i j}^{+}, t_{i j}, t_{i j}^{+}, g_{0}^{i}\right)$ generators of the massive HS symmetry superalgebra $\mathcal{A}^{f}\left(Y(k), \mathbb{R}^{1, d-1}\right)$ satisfies the same algebraic relations as those in Table 1 and Table 2 for the massless HS symmetry superalgebra, except for the commutators

$$
\left[t_{i}, l_{j}^{+}\right]=-\delta_{i j}\left(\tilde{t}_{0}-\tilde{\gamma} m\right), \quad\left[t_{i}^{+}, l_{j}\right]=\delta_{i j}\left(\tilde{t}_{0}-\tilde{\gamma} m\right), \quad\left[l_{i}, l_{j}^{+}\right]=\delta_{i j}\left(\tilde{l}_{0}-m^{2}\right)
$$

Definitions (3.20), (3.21) and relations (3.26) show the presence of $2 k$ additional second-class constraints, $l_{i}, l_{i}^{+}$, with the corresponding oscillator operators $b_{i}, b_{i}^{+},\left[b_{i}, b_{j}^{+}\right]=\delta_{i j}$, in comparison with the massless case.

It is interesting to observe that the elements with a tilde in Eqs.(3.21)-(3.24) satisfy the algebraic relations for the massless HS symmetry superalgebra $\mathcal{A}^{f}\left(Y(k), \mathbb{R}^{1, d-1}\right)$ now without a central charge (i.e., the quantities $\tilde{o}_{I}$ contain the same second-class constraints as $o_{I}$ in the massless case), however, in a wider (than $\mathcal{H}$ ) Fock space $\mathcal{H} \otimes \mathcal{H}\left(b_{i}, b_{i}^{+}\right)$, with a tensor co-multiplier $\mathcal{H}\left(b_{i}, b_{i}^{+}\right)$ generated by the "massive" oscillator $b_{i}, b_{i}^{+}$. Therefore, the converted constraints $O_{I}, O_{I}=o_{I}+o_{I}^{\prime}$, in the massive case are given by the relations

$$
O_{I}=\tilde{o}_{I}+o_{I}^{\prime}, \quad M=m+m^{\prime}=0,
$$

where the additional parts $o_{I}^{\prime}=o_{I}^{\prime}\left(f_{i}, f_{i}^{+} ; b_{i j}, b_{i j}^{+}, d_{i_{1} j_{1}}, d_{i_{1} j_{1}}^{+}\right)$are determined by relations (3.1)(3.9).

Thus, the auxiliary representation (generalized Verma module) for the $\operatorname{spp}(1 \mid 2 k)$ superalgebra completely determines, with the help of dimensional reduction, an oscillator realization for the additional parts of the massive HS symmetry superalgebra $\mathcal{A}^{\prime f}\left(Y(k), \mathbb{R}^{1, d-1}\right)$.

In the following section, we determine the superalgebra of the extended constraints and find the BRST operator corresponding to this superalgebra.

\section{BRST-BFV Operator}

Now, we are in a position to find the BRST-BFV operator for the Lie superalgebra of converted constraints $O_{I}$, following our approach. Because the algebra under consideration is a Lie superalgebra $\mathcal{A}^{f}\left(Y(k), \mathbb{R}^{1, d-1}\right)$, this operator can be constructed according to the standard prescription [36]. To this end, we introduce the set of ghost fields $C^{I}=\left(q_{0}, q_{i}, q_{i}^{+} ; \eta_{0}, \eta^{i}, \eta_{i}^{+}, \eta^{i j}, \eta_{i j}^{+}, \vartheta_{r s}, \vartheta_{r s}^{+}\right.$, $\left.\eta_{G}^{i}\right)$ with the Grassmann parity opposite to that of the elements $O_{I}=\left(T_{0}, T_{i}^{+}, T_{i} ; L_{0}, L_{i}^{+}, L_{i}, L_{i j}\right.$, $\left.L_{i j}^{+}, T_{i j}, T_{i j}^{+}, G_{0}^{i}\right)_{8}^{8}$, subject to the properties

$$
\left(\eta^{i j}, \eta_{i j}^{+}\right)=\left(\eta^{j i}, \eta_{j i}^{+}\right), \quad\left(\vartheta_{r s}, \vartheta_{r s}^{+}\right)=\left(\vartheta_{r s}, \vartheta_{r s}^{+}\right) \theta^{s r}
$$

and their conjugated ghost momenta $\mathcal{P}_{I}$ with the same properties as those for $C^{I}$ in (4.1) with the only nonvanishing commutation relations for bosonic ghosts

$$
\left[q_{i}, p_{j}^{+}\right]=\left[p_{i}, q_{j}^{+}\right]=\delta_{i j}, \quad\left[q_{0}, p_{0}\right]=\imath ;
$$

\footnotetext{
${ }^{8}$ For massless HS fields, the elements $T_{0}, L_{0}, L_{i}^{+}, L_{i}$ coincide with $t_{0}, l_{0}, l_{i}^{+}, l_{i}$, whereas in the massive case $T_{0}=\tilde{t}_{0}, L_{0}=\tilde{l}_{0}, L_{i}^{+}=l_{i}^{+}+l_{i}^{\prime+}, L_{i}=l_{i}+l_{i}^{\prime}$, with allowance for Eqs. (3.20), (3.21), (3.22)
} 
and the anticommutation ones for fermionic ghosts

$$
\begin{array}{ll}
\left\{\vartheta_{r s}, \lambda_{t u}^{+}\right\}=\left\{\lambda_{t u}, \vartheta_{r s}^{+}\right\}=\delta_{r t} \delta_{s u}, & \left\{\eta_{i}, \mathcal{P}_{j}^{+}\right\}=\left\{\mathcal{P}_{j}, \eta_{i}^{+}\right\}=\delta_{i j}, \\
\left\{\eta_{l m}, \mathcal{P}_{i j}^{+}\right\}=\left\{\mathcal{P}_{i j}, \eta_{l m}^{+}\right\}=\delta_{l i} \delta_{j m}, & \left\{\eta_{0}, \mathcal{P}_{0}\right\}=\imath,\left\{\eta_{\mathcal{G}}^{i}, \mathcal{P}_{\mathcal{G}}^{j}\right\}=\imath \delta^{i j} .
\end{array}
$$

The ghost coordinates and momenta also possess the standard ghost number distribution, $g h\left(\mathcal{C}^{I}\right)$ $=-g h\left(\mathcal{P}_{I}\right)=1$, providing the property $g h\left(Q^{\prime}\right)=1$, and posses the Hermitian conjugation properties of zero-mode pairs, 9

$$
\left(q_{0}, \eta_{0}, \eta_{G}^{i}, p_{0}, \mathcal{P}_{0}, \mathcal{P}_{G}^{i}\right)^{+}=\left(q_{0}, \eta_{0}, \eta_{G}^{i}, p_{0},-\mathcal{P}_{0},-\mathcal{P}_{G}^{i}\right)
$$

The BRST operator for the algebra of $O_{I}$, given by Tables 1, 2, can be found in an exact form, with the use of the $(\mathcal{C P})$-ordering for the operators of ghost coordinates $\mathcal{C}^{I}$ and momenta $\mathcal{P}_{I}$, as follows:

$$
Q^{\prime}=O_{I} \mathcal{C}^{I}+\frac{1}{2} \mathcal{C}^{I} \mathcal{C}^{J} f_{J I}^{K} \mathcal{P}_{K}(-1)^{\varepsilon\left(O_{K}\right)+\varepsilon\left(O_{I}\right)}
$$

with the constants $f_{I J}^{K}(2.20)$ written in a compact $x$-local representation. According to Tables 1, 2 $Q^{\prime}$, we finally have

$$
\begin{aligned}
& Q^{\prime}= \frac{1}{2} q_{0} T_{0}+q_{i}^{+} T^{i}+\frac{1}{2} \eta_{0} L_{0}+\eta_{i}^{+} L^{i}+\sum_{l \leq m} \eta_{l m}^{+} L^{l m}+\sum_{l<m} \vartheta_{l m}^{+} T^{l m}+\frac{1}{2} \eta_{G}^{i} G_{i} \\
&+\left[\frac{1}{2} \sum_{l, m}\left(1+\delta_{l m}\right) \eta^{l m} q_{l}^{+}-\sum_{l<m} q_{l} \vartheta^{l m}-\sum_{m<l} q_{l} \vartheta^{m l+}\right] p_{m}^{+}+\frac{1}{2} \sum_{m} \eta_{G}^{m}\left(q_{m} p_{m}^{+}+q_{m}^{+} p_{m}\right) \\
&+\imath \sum_{l}\left[\frac{1}{2} \eta_{l}^{+} \eta^{l} \mathcal{P}_{0}+\eta_{l}^{+} q^{l} p_{0}-q^{l} q_{l}^{+} \mathcal{P}_{G}^{l}\right]-\frac{\imath}{2} q_{0}^{2} \mathcal{P}_{0} \\
&-\sum_{i<l<j} \vartheta_{l j}^{+} \vartheta_{i}^{+l} \lambda^{i j}+\frac{\imath}{2} \sum_{l<m} \vartheta_{l m}^{+} \vartheta^{l m}\left(\mathcal{P}_{G}^{m}-\mathcal{P}_{G}^{l}\right)-\sum_{l<n<m} \vartheta_{l m}^{+} \vartheta_{n}^{l} \lambda^{n m} \\
&+\sum_{n<l<m} \vartheta_{l m}^{+} \vartheta_{n}^{m} \lambda^{+n l}-\sum_{n, l<m}\left(1+\delta_{l n}\right) \vartheta_{l m}^{+} \eta^{l+} \mathcal{P}_{n n}^{m n}+\sum_{n, l<m}\left(1+\delta_{m n}\right) \vartheta_{l m}^{+} \eta^{m}{ }_{n} \mathcal{P}^{+l n} \\
&+\frac{\imath}{8} \sum_{l \leq m}\left(1+\delta_{l m}\right) \eta_{l m}^{+} \eta^{l m}\left(\mathcal{P}_{G}^{l}+\mathcal{P}_{G}^{m}\right)+\frac{1}{2} \sum_{l \leq m}\left(1+\delta_{l m}\right) \eta_{G}^{l}\left(\eta_{l m}^{+} \mathcal{P}^{l m}-\eta_{l m} \mathcal{P}^{l m+}\right) \\
&+\frac{1}{2} \sum_{l<m, n \leq m} \eta_{n m}^{+} \eta_{l}^{n} \lambda^{l m}-2 \sum_{l<m} q_{l} q_{m}^{+} \lambda^{l m}+\frac{1}{2} \sum_{l<m}\left(\eta_{G}^{m}-\eta_{G}^{l}\right)\left(\vartheta_{l m}^{+} \lambda^{l m}-\vartheta_{l m} \lambda^{l m+}\right) \\
&-\left[\frac{1}{2} \sum_{l, m}\left(1+\delta_{l m}\right) \eta^{m} \eta_{l m}^{+}+\sum_{l<m} \vartheta_{l m} \eta^{+m}+\sum_{m<l} \vartheta_{m l}^{+} \eta^{+m}+2 \sum_{l} q_{0} q_{l}^{+}\right] \mathcal{P}^{l} \\
&-2 \sum_{l, m} q_{l}^{+} q_{m}^{+} \mathcal{P}^{l m}+\frac{1}{2} \sum_{l} \eta_{G}^{l}\left(\eta_{l}^{+} \mathcal{P}^{l}-\eta_{l} \mathcal{P}^{l+}\right)+h . c . \\
&
\end{aligned}
$$

In connection with the representation of the BRST operator (4.6), note, first, that a nilpotent $Q^{\prime}$ has a matrix-like $2^{\left[\frac{d}{2}\right]} \times 2^{\left[\frac{d}{2}\right]}$ structure (providing its correct action on a Dirac spinor as the stringvector $|\Psi\rangle$, (2.6) , however, extended to the total Hilbert space $\left.\mathcal{H}_{t o t}=\mathcal{H} \otimes \mathcal{H}^{\prime} \otimes \mathcal{H}_{g h}\right)$. Second, it can be presented as the sum of the BRST operator $Q_{b}^{\prime}$, corresponding to the symplectic $s p(2 k)$ algebra, and an additional term, $Q_{f}^{\prime}$, vanishing for $\left(q_{0}, q_{i}, q_{i}^{+}, p_{0}, p_{i}, p_{i}^{+}\right)=0$ :

$$
Q^{\prime}=Q_{b}^{\prime}+Q_{f}^{\prime},\left.Q_{f}^{\prime}\right|_{\left(q_{0}, q_{i}, q_{i}^{+}, p_{0}, p_{i}, p_{i}^{+}\right)=0=0}
$$

\footnotetext{
${ }^{9}$ By means of the redefinition $\left(p_{i}, \mathcal{P}_{0}, \mathcal{P}_{G}^{i}\right) \mapsto \imath\left(p_{i}, \mathcal{P}_{0}, \mathcal{P}_{G}^{i}\right)$, the BRST operator (4.6) and relations (4.2), (4.3) are written in the notation of [55], [56].
} 
where the specific form of $Q_{b}^{\prime}$ easily follows from Eq. (4.6), with allowance for the boundary condition (4.7), and was already presented in [31, however without the fermionic oscillators $f_{i}, f_{i}^{+}$, i.e., for $\left(f_{i}, f_{i}^{+}\right)=0$. As in the case of bosonic HS fields [31], the property of the BRST operator to be Hermitian is defined by the same rule

$$
Q^{\prime+} K=K Q^{\prime}, \quad K=\hat{1} \otimes K^{\prime} \otimes \hat{1}_{g h}
$$

and is calculated with respect to the odd scalar product $\langle\mid\rangle$ in $\mathcal{H}_{t o t}$ with the measure $d^{d} x$, which, in its turn, is constructed as the direct product of odd scalar products in $\mathcal{H}$ and even ones in $\mathcal{H}^{\prime}$ and $\mathcal{H}_{g h}$. The operator $K$ in (4.8) is the tensor product of the operator $K^{\prime}$ in $\mathcal{H}^{\prime}(3.16)$ and the unit operators in $\mathcal{H}, \mathcal{H}_{g h}$.

We have thus constructed a Hermitian BRST operator for the superalgebra $\mathcal{A}_{c}^{f}\left(Y(k), \mathbb{R}^{1, d-1}\right)$ of converted operators $O_{I}$. In the following section, we will use this operator to construct a Lagrangian action for fermionic HS fields of spin $\left(n_{1}+\frac{1}{2}, \ldots, n_{k}+\frac{1}{2}\right)$ in a flat space-time.

\section{Unconstrained Gauge-invariant Lagrangians}

Let us develop a Lagrangian formulation for fermionic higher-spin fields in a $d$-dimensional Minkowski space, partially following the algorithm of [59], which is a particular case of our construction, corresponding to a Young tableaux with 2 rows. In the beginning, one should extract the dependence of the BRST operator $Q^{\prime}$ (4.6) on the "particle number" of ghosts $\eta_{G}^{i}, \mathcal{P}_{G}^{i}$, in order to obtain the BRST operator $Q$ only for the system of converted first-class constraints $\left\{O_{I}\right\} \backslash\left\{\mathcal{G}_{0}^{i}\right\}$ (to be nilpotent after being restricted to the corresponding Hilbert subspaces):

$$
Q^{\prime}=Q+\eta_{G}^{i}\left(\sigma^{i}+h^{i}\right)+\mathcal{B}^{i} \mathcal{P}_{G}^{i},
$$

where the generalized spin operator $\vec{\sigma}=\left(\sigma^{1}, \sigma^{2}, \ldots, \sigma^{k}\right)$, extended by the ghost Wick-pair variables is Hermitian, $\sigma^{i+} K=K \sigma^{i}$, and reads

$$
\begin{aligned}
\sigma^{i}= & G_{0}^{i}-h^{i}-\eta_{i} \mathcal{P}_{i}^{+}+\eta_{i}^{+} \mathcal{P}_{i}+\sum_{m}\left(1+\delta_{i m}\right)\left(\eta_{i m}^{+} \mathcal{P}^{i m}-\eta_{i m} \mathcal{P}_{i m}^{+}\right) \\
& +\sum_{l<i}\left[\vartheta_{l i}^{+} \lambda^{l i}-\vartheta^{l i} \lambda_{l i}^{+}\right]-\sum_{i<l}\left[\vartheta_{i l}^{+} \lambda^{i l}-\vartheta^{i l} \lambda_{i l}^{+}\right]+q_{i} p_{i}^{+}+q_{i}^{+} p_{i}
\end{aligned}
$$

The operator $Q$ in Eq. (15.1) (not yet nilpotent in $\mathcal{H}_{\text {tot }}$ ) corresponds to a system of converted first-class constraints and is unambiguously determined as

$$
\begin{aligned}
Q= & \frac{1}{2} q_{0} T_{0}+q_{i}^{+} T^{i}+\frac{1}{2} \eta_{0} L_{0}+\eta_{i}^{+} L^{i}+\sum_{l \leq m} \eta_{l m}^{+} L^{l m}+\sum_{l<m} \vartheta_{l m}^{+} T^{l m}+\frac{\imath}{2}\left(\sum_{l} \eta_{l}^{+} \eta^{l}-q_{0}^{2}\right) \mathcal{P}_{0} \\
& +\left[\frac{1}{2} \sum_{l, m}\left(1+\delta_{l m}\right) \eta^{l m} q_{l}^{+}-\sum_{l<m} q_{l} \vartheta^{l m}-\sum_{m<l} q_{l} \vartheta^{m l+}\right] p_{m}^{+}+\imath \sum_{l} \eta_{l}^{+} q^{l} p_{0}-2 \sum_{l<m} q_{l} q_{m}^{+} \lambda^{l m} \\
& -2 \sum_{l, m} q_{l}^{+} q_{m}^{+} \mathcal{P}^{l m}-\sum_{i<l<j} \vartheta_{l j}^{+} \vartheta_{i}^{+l} \lambda^{i j}-\sum_{l<n<m} \vartheta_{l m}^{+} \vartheta_{n}^{l} \lambda^{n m}+\sum_{n<l<m} \vartheta_{l m}^{+} \vartheta_{n}{ }^{m} \lambda^{+n l} \\
& -\sum_{n, l<m}\left(1+\delta_{l n}\right) \vartheta_{l m}^{+} \eta^{l+}{ }_{n} \mathcal{P}^{m n}+\sum_{n, l<m}\left(1+\delta_{m n}\right) \vartheta_{l m}^{+} \eta^{m}{ }_{n} \mathcal{P}^{+l n}+\frac{1}{2} \sum_{l<m, n \leq m} \eta_{n m}^{+} \eta^{n}{ }_{l} \lambda^{l m} \\
& -\left[\frac{1}{2} \sum_{l, m}\left(1+\delta_{l m}\right) \eta^{m} \eta_{l m}^{+}+\sum_{l<m} \vartheta_{l m} \eta^{+m}+\sum_{m<l} \vartheta_{m l}^{+} \eta^{+m}+2 \sum_{l} q_{0} q_{l}^{+}\right] \mathcal{P}^{l}+h . c .
\end{aligned}
$$

The operator $Q$ (modulo its spinor nature) contains - for vanishing fermionic oscillators $\left(f_{i}, f_{i}^{+}\right)$ and bosonic ghosts $\left(q_{0}, q_{i}, q_{i}^{+} ; p_{0}, p_{i}^{+}, p_{i}\right)$, for $i=1, \ldots, k$ - the BRST operator $Q_{b}$ for the converted 
first-class constraints corresponding to the HS symmetry algebra $\mathcal{A}_{c}\left(Y(k), \mathbb{R}^{1, d-1}\right)$ for bosonic HS fields, obtained earlier [31], in correspondence with the representation (4.7) for $Q^{\prime}$. Finally, the quantities $\mathcal{B}^{i}$ in (5.1) are uniquely determined by Eq. (4.6) as follows:

$$
\mathcal{B}^{i}=-2 \imath p \sum_{l} q^{l} q_{l}^{+}-\imath \sum_{l<m} \vartheta_{l m}^{+} \vartheta^{l m}\left(\delta^{m i}-\delta^{l i}\right)+\frac{\imath}{4} \sum_{l \leq m}\left(1+\delta_{l m}\right) \eta_{l m}^{+} \eta^{l m}\left(\delta^{i l}+\delta^{m i}\right) .
$$

By construction, from the nilpotency of the BRST operator $Q^{\prime}$, considered in powers of the ghosts $\left(\eta_{G}^{i}, \mathcal{P}_{G}^{i}\right)$, the set of operators $Q, \sigma^{i}, \mathcal{B}^{i}$ supercommutes with each other,

$$
\left[Q, \sigma^{i}\right\}=0, \quad\left[Q, \mathcal{B}^{i}\right\}=0, \quad\left[\sigma^{i}, \mathcal{B}^{j}\right\}=0, \text { for } i, j=1, \ldots, k
$$

providing an equation for $Q^{2}$ :

$$
Q^{\prime 2}=0 \Longleftrightarrow Q^{2}=-\imath \sum_{i} \mathcal{B}^{i} \sigma^{i}
$$

We then choose the standard representation for the Hilbert space $\mathcal{H}_{\text {tot }}$ :

$$
\left(q_{i}, \eta_{i}, \eta_{i j}, \vartheta_{r s}, p_{0}, p_{i}, \mathcal{P}_{0}, \mathcal{P}_{i}, \mathcal{P}_{i j}, \lambda_{r s}, \mathcal{P}_{G}^{i}\right)|0\rangle=0, \quad|0\rangle \in \mathcal{H}_{t o t}
$$

under the assumption that the field vectors $|\chi\rangle$, as well as the gauge parameters $|\Lambda\rangle$ (Dirac spinors), do not depend on the ghosts $\eta_{G}^{i}$ for particle number operators $G_{0}^{i}$,

$$
\begin{aligned}
|\chi\rangle= & \sum_{n} \prod_{c}^{k}\left(f_{c}^{+}\right)^{n_{c}^{0}} \prod_{l}^{k}\left(b_{l}^{+}\right)^{n_{l}^{\prime}} \prod_{i \leq j, r<s}^{k}\left(b_{i j}^{+}\right)^{n_{i j}}\left(d_{r s}^{+}\right)^{p_{r s}} q_{0}^{n_{b 0}} \eta_{0}^{n_{f 0}} \\
& \times \prod_{e, g, i, j, l \leq m, n \leq o}\left(q_{e}^{+}\right)^{n_{a e}}\left(p_{g}^{+}\right)^{n_{b g}}\left(\eta_{i}^{+}\right)^{n_{f i}}\left(\mathcal{P}_{j}^{+}\right)^{n_{p j}}\left(\eta_{l m}^{+}\right)^{n_{f l m}}\left(\mathcal{P}_{n o}^{+}\right)^{n_{p n o}} \prod_{r<s, t<u}\left(\vartheta_{r s}^{+}\right)^{n_{f r s}}\left(\lambda_{t u}^{+}\right)^{n_{\lambda t u}} \\
& \times \mid \Psi\left(a_{i}^{+}\right)_{\left(n^{0}\right)_{c} ;\left(n^{\prime}\right)_{l}(n)_{i j}(p)_{r s}}^{\left.n_{b i}(n)_{p j}(n)_{f l m}(n)_{p n o}(n)_{f r s}(n)_{\lambda t u}\right\rangle}
\end{aligned}
$$

The brackets $\left(n^{0}\right)_{c},(n)_{f i},(n)_{p j},(n)_{i j}$ in the definition of (5.8) imply, for instance, for $\left(n^{0}\right)_{c}$ and $(n)_{i j}$, the sets of indices $\left(n_{1}^{0}, \ldots, n_{k}^{0}\right)$ and $\left(n_{11}, \ldots, n_{1 k}, \ldots, n_{k 1}, \ldots, n_{k k}\right)$. The above sum is taken over $n_{b 0}, n_{a e}, n_{b g}, h_{l}, n_{i j}, p_{r s}$, running from 0 to infinity, and over the remaining $n$ 's from 0 to 1 , whereas

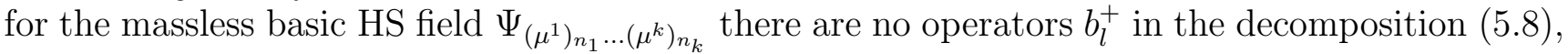
i.e., $\left(n^{\prime}\right)_{l}=(0)_{l}$. We denote by $\left|\chi^{k}\right\rangle$ the state (5.8) with the ghost number $-k$, i.e., $g h\left(\left|\chi^{k}\right\rangle\right)=-k$. Thus, the physical state having the zero ghost number is $\left|\chi^{0}\right\rangle$; the gauge parameters $|\Lambda\rangle$ having the ghost number -1 is $\left|\chi^{1}\right\rangle$, and so on. Moreover, for the vanishing of all auxiliary creation operators $f^{+}, b^{+}, d^{+}$and ghost variables $q_{0}, q_{i}^{+}, \eta_{0}, \eta_{i}^{+}, p_{i}^{+}, \mathcal{P}_{i}^{+}, \ldots$, the vector $\left|\chi^{0}\right\rangle$ should contain only the physical string-like vector $|\Psi\rangle=\left|\Psi\left(a_{i}^{+}\right)_{\left(0^{0}\right)_{c} ;(0)_{l}(0)_{i j}(0)_{r s}}^{0_{b 0} 0_{f i} ;(0)_{p j}(0)_{f l m}(0)_{p n o}(0)_{f r s}(0)_{\lambda t u}}\right\rangle$, so that

$$
\left|\chi^{0}\right\rangle=|\Psi\rangle+\left|\Psi_{A}\right\rangle,\left.\quad\left|\Psi_{A}\right\rangle\right|_{\left(f^{+}, b^{+}, d^{+}, q_{0}, q_{i}^{+}, \eta_{0}, \eta_{i}^{+}, p_{i}^{+}, \mathcal{P}_{i}^{+}, \ldots\right)=0}=0
$$

with the vector $\left|\Psi_{A}\right\rangle$ containing only the set of auxiliary spin-tensors as its components. We will show in Appendix C that the vector $\left|\Psi_{A}\right\rangle$ can be completely gauged away, by using a partial gauge fixing and solving some of the equations of motion.

Next, we derive, from the BRST-like equation, determining the physical vector, $Q^{\prime}|\chi\rangle=0$, (for $\left.|\chi\rangle=\left|\chi^{0}\right\rangle\right)$ and from the set of reducible gauge transformations, $\delta|\chi\rangle=Q^{\prime}|\Lambda\rangle, \delta|\Lambda\rangle=Q^{\prime}\left|\Lambda^{(1)}\right\rangle$, 
$\ldots, \delta\left|\Lambda^{(s-1)}\right\rangle=Q^{\prime}\left|\Lambda^{(s)}\right\rangle$, a sequence of relations underlying the $\eta_{G}^{i}$-independence of all of the above vectors:

$$
\begin{array}{lll}
Q|\chi\rangle=0, & \left(\sigma^{i}+h^{i}\right)|\chi\rangle=0, & (\varepsilon, g h)(|\chi\rangle)=(1,0), \\
\delta|\chi\rangle=Q|\Lambda\rangle, & \left(\sigma^{i}+h^{i}\right)|\Lambda\rangle=0, & (\varepsilon, g h)(|\Lambda\rangle)=(0,-1), \\
\delta|\Lambda\rangle=Q\left|\Lambda^{(1)}\right\rangle, & \left(\sigma^{i}+h^{i}\right)\left|\Lambda^{(1)}\right\rangle=0, & (\varepsilon, g h)\left(\left|\Lambda^{(1)}\right\rangle\right)=(1,-2), \\
\delta\left|\Lambda^{(s-1)}\right\rangle=Q\left|\Lambda^{(s)}\right\rangle, & \left(\sigma^{i}+h^{i}\right)\left|\Lambda^{(s)}\right\rangle=0, & (\varepsilon, g h)\left(\left|\Lambda^{(s)}\right\rangle\right)=(s \quad \bmod 2,-s-1) .
\end{array}
$$

In the above equations, $s=\sum_{l=1}^{k} n_{l}+k(k-1) / 2-1$ is the stage of reducibility for both massless and massive fermionic HS field, since the only non-vanishing vector (independent gauge parameter $\left|\Lambda^{s}\right\rangle$ ) has the lowest negative ghost number, when, for instance, all powers of commuting ghost momenta $\left(p_{i}^{+}\right)$and all "mixed-symmetry" fermionic ghost momenta $\lambda_{r s}^{+}$compose $\left|\Lambda^{s}\right\rangle$, without the ghost coordinates $\mathcal{C}^{I}$ in this vector. The solution of the spectral problem given by Eqs. (5.10)(5.13) is compatible due to the validity of the second group in the set of supercommutators (5.5), and is described by the solution of the second column there. The middle set of equations (5.10)(5.13), with the generalized spin operator $\sigma^{i}$, determines the set of proper eigenvectors $\left|\chi^{0}\right\rangle_{(m)_{k}}$, $\left|\chi^{1}\right\rangle_{(m)_{k}}, \ldots,\left|\chi^{s}\right\rangle_{(m)_{k}}, m_{1} \geq m_{2} \geq \ldots m_{k} \geq 0$, and the set of the corresponding eigenvalues for possible values of the parameters $h^{i}$,

$$
-h^{i}=m^{i}+\frac{d-4 i}{2}, \quad i=1, . ., k, \quad m_{1}, \ldots, m_{k-1} \in \mathbb{Z}, m_{k} \in \mathbb{N}_{0}
$$

for massless,

$$
-h_{m}^{i}=m^{i}+\frac{d+1-4 i}{2}, \quad i=1, . ., k, \quad m_{1}, \ldots, m_{k-1} \in \mathbb{Z}, m_{k} \in \mathbb{N}_{0}
$$

and for massive half-integer HS fields. The values of $m_{i}$ are related to the spin components $s_{i}=n_{i}+\frac{1}{2}$ of the initial spin-tensor (2.1) , because the proper vector $|\chi\rangle_{\left(n_{1}, \ldots, n_{k}\right)}$, corresponding to $\left(h_{1}, \ldots, h_{k}\right)$ has the leading term

$$
\left|\Psi\left(a_{i}^{+}\right)_{\left(0^{0}\right)_{c} ;(0)_{l}(0)_{i j}(0)_{r s}}^{0_{b 0} 0_{f 0} ;(0)_{a e}(0)_{b g}(0)_{f i}(0)_{p j}(0)_{f l m}(0)_{p n o}(0)_{f r s}(0)_{\lambda t u}}\right\rangle
$$

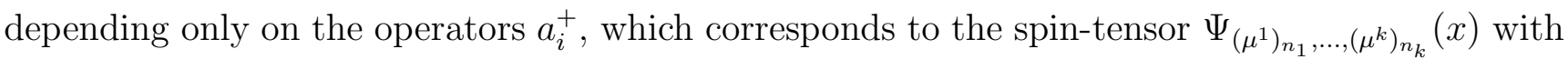
the initial value of $\operatorname{spin} \mathbf{s}=\left(s_{1}+\frac{1}{2}, \ldots, s_{k}+\frac{1}{2}\right)$ in the decomposition (5.8) and representation (5.9) ). Let us denote by $|\chi\rangle_{(m p)_{k}}$ the eigenvectors of $\sigma_{i}$ corresponding to the eigenvalues $\left(m^{i}+\frac{d-4 i}{2}\right)$. Therefore, we conclude that

$$
\sigma_{i}|\chi\rangle_{(m)_{k}}=\left(m^{i}+\frac{d+\Theta(m)-4 i}{2}\right)|\chi\rangle_{(m)_{k}},
$$

jointly for massless $(m=\theta(m)=0)$ and massive $(m \neq 0 \Longrightarrow \theta(m)=1)$ fermionic HS fields with the help of the Heaviside $\theta$-function. We can show that, in order to construct a Lagrangian for the field corresponding to a definite Young tableau (2.1), the numbers $m_{i}$ must be equal to the numbers of the cells in the $i$-th row of the corresponding Young tableau, i.e., $m_{i}=n_{i}$. Therefore, the state $|\chi\rangle_{(n)_{k}}$ contains the physical field (2.6) and all of its auxiliary fields. Let us fix some values of $m_{i}=n_{i}$. Then, one has to substitute $h_{i}$ corresponding to the chosen $n_{i}$ (5.14) or (5.15) into (5.3) , (5.10) -(5.13). Thus, the equation of motion (5.10) corresponding to the field with a given spin $\left(n_{1}+\frac{1}{2}, \ldots, n_{k}+\frac{1}{2}\right)$ has the form

$$
Q_{(n)_{k}}\left|\chi^{0}\right\rangle_{(n)_{k}}=0
$$


where the ordered value of spin $n_{1} \geq n_{2} \geq \ldots \geq n_{k}$ for the vector $\left|\chi^{l}\right\rangle_{(n)_{k}}$ should be composed from the set of integers $\left(n_{b 0}, n_{f 0}, n_{a e}, n_{b g}, n_{c}^{0}, n_{l}^{\prime}, n_{i j}, p_{r s}, n_{f i}, n_{p j}, n_{f l m}, n_{p n o}, n_{f r s}, n_{\lambda t u}, p_{i}\right)$, for $e, g, c, l, i, j, r, s, l, m, n, o, t, u=1, \ldots, k, i \leq j, r<s, l \leq m, n \leq o, t<u$; in (5.8) and (2.6) in the decomposition (5.8) the coefficient. 10 are to be restricted for all the vectors $\left|\chi^{l}\right\rangle_{(n)_{k}}$, $l=0, \ldots, \sum_{o=1}^{k} n_{o}+k(k-1) / 2-1$, in view of the solution for the spectral problem (5.16), by the formulae

$$
\begin{aligned}
n_{i}= & p_{i}+\Theta(m) n_{i}^{\prime}+n_{a i}+n_{b i}+n_{i}^{0}+\sum_{j=1}\left(1+\delta_{i j}\right)\left(n_{i j}+n_{f i j}+n_{p i j}\right)+n_{f i}+n_{p i} \\
& +\sum_{r<i}\left(p_{r i}+n_{f r i}+n_{\lambda r i}\right)-\sum_{r>i}\left(p_{i r}+n_{f i r}+n_{\lambda i r}\right), i=1, \ldots, k .
\end{aligned}
$$

In addition to the restrictions (5.18), valid for a general case of HS fields subject to $Y\left(s_{1}, \ldots, s_{k}\right)$, the subset of "ghost" numbers $\left(n_{f 0}, n_{f i}, n_{b 0}, n_{a e}, n_{b g}, n_{p j}, n_{f l m}, n_{p n o}, n_{f r s}, n_{\lambda t u}\right)$ in (5.8) and (2.6) for fixed values of $n_{i}$, satisfies the following equations for $\left|\chi^{l}\right\rangle_{(n)_{k}}, l=0, \ldots, \sum_{o=1}^{k} n_{o}+k(k-1) / 2$ (with the identification $\left|\chi^{l}\right\rangle_{(n)_{k}}=\left|\Lambda^{l-1}\right\rangle_{(n)_{k}}$ for $l>0$ ):

$$
\left|\chi^{l}\right\rangle_{(n)_{k}}: n_{b 0}+n_{f 0}+\sum_{i}\left(n_{f i}-n_{p i}+n_{a i}-n_{b i}\right)+\sum_{i \leq j}\left(n_{f i j}-n_{p i j}\right)+\sum_{r<s}\left(n_{f r s}-n_{\lambda r s}\right)=-l(5
$$

Since the BRST-BFV operator $Q^{\prime}$ is nilpotent (4.6) for any values of $h_{i}$, and, due to the proportionality of $Q^{2}$ (5.6) to the generalized spin operator, and because of a joint solution of the spectral problem (5.10)-(5.13), we have a sequence of reducible gauge transformations:

$$
\begin{aligned}
& \delta\left|\chi^{0}\right\rangle_{(n)_{k}}=Q_{(n)_{k}}|\Lambda\rangle_{(n)_{k}}, \quad \delta|\Lambda\rangle_{(n)_{k}}=Q_{(n)_{k}}\left|\Lambda^{(1)}\right\rangle_{(n)_{k}}, \\
& \text {... } \\
& \delta\left|\Lambda^{(s-1)}\right\rangle_{(n)_{k}}=Q_{(n)_{k}}\left|\Lambda^{(s)}\right\rangle_{(n)_{k}}, \quad \delta\left|\Lambda^{(s)}\right\rangle_{(n)_{k}}=0, s=\sum_{o=1}^{k} n_{o}+k(k-1) / 2-1
\end{aligned}
$$

with a nilpotent $Q_{(n)_{k}}$ in its action on proper eigenfunctions of the operator $\sigma^{i},|\chi\rangle_{(n)_{k}}$ :

$$
Q_{(n)_{k}}^{2}\left(|\chi\rangle_{(n)_{k}},|\Lambda\rangle_{(n)_{k}}, \ldots,\left|\Lambda^{\left(\sum_{o=1}^{k} n_{o}+k(k-1) / 2-1\right)}\right\rangle_{(n)_{k}}\right) \equiv 0 .
$$

Summarizing the above, we have obtained the equations of motion (5.17) for an arbitrary halfinteger-spin gauge theory subject to $Y T\left(s_{1}, \ldots, s_{k}\right)$ with a mixed symmetry in any space-time dimension, as well as the tower of reducible gauge transformations (5.20)-(5.21). An essential point is that these equations are more than first order in the space-time derivatives $\partial_{\mu}$, due to the presence of $L_{0} \sim \partial^{2}$ in the operator $Q^{11}$.

To obtain the Lagrangian formulation with first-order derivatives only, we use the functional dependence of the operator $L_{0}$ on a fermionic operator $T_{0}, L_{0}=-T_{0}^{2}$ and attempt to gauged away the dependence on $L_{0}, \eta_{0}$ from the BRST operator $Q$ (5.3) and from the whole set of the vectors $\left|\chi^{l}\right\rangle_{(n)_{k}}$. To this end, we extract the zero-mode ghosts from the operator $Q$ as follows:

$$
Q=q_{0} \tilde{T}_{0}+\eta_{0} L_{0}+\imath\left(\eta_{i}^{+} q_{i}-\eta_{i} q_{i}^{+}\right) p_{0}-\imath\left(q_{0}^{2}-\eta_{i}^{+} \eta_{i}\right) \mathcal{P}_{0}+\Delta Q
$$

\footnotetext{
${ }^{10}$ We replace the indices $n_{i}$ in (2.6) for a vector of the initial Fock space $\mathcal{H}$ by $p_{i}$ due to the use of $n_{i}$ in the value of generalized spin for the basic HS field $\Psi_{\left(\mu^{1}\right)_{n_{1}}, \ldots,\left(\mu^{k}\right)_{n_{k}}}$

${ }^{11}$ Formally, we are able to derive the equations of motion (5.17), as in the case of bosonic mixed-symmetric HS fields [31, from the Lagrangian action $\Pi \mathcal{S}_{(n)_{k}}, \Pi \mathcal{S}_{(n)_{k}}=\int d \eta_{0}(n)_{k}\left\langle\tilde{\chi}^{0}\left|K_{(n)_{k}} Q_{(n)_{k}}\right| \chi^{0}\right\rangle_{(n)_{k}}$, with the operator $K$ (4.8), having in mind the peculiarity found in the research on superfield Lagrangian BRST quantization (e.g., 39, [40, [4] $)$, that the action $\Pi \mathcal{S}_{(n)_{k}}$ is an odd-valued quantity, $\varepsilon\left(\Pi \mathcal{S}_{(n)_{k}}\right)=1$.
} 
where

$$
\begin{aligned}
\tilde{T}_{0}= & T_{0}-2 q_{i}^{+} \mathcal{P}_{i}-2 q_{i} \mathcal{P}_{i}^{+} \\
\Delta Q= & q_{i}^{+} T^{i}+\eta_{i}^{+} L^{i}+\sum_{l \leq m} \eta_{l m}^{+} L^{l m}+\sum_{l<m} \vartheta_{l m}^{+} T^{l m}+\left[\frac{1}{2} \sum_{l, m}\left(1+\delta_{l m}\right) \eta^{l m} q_{l}^{+}\right. \\
& \left.-\sum_{l<m} q_{l} \vartheta^{l m}-\sum_{m<l} q_{l} \vartheta^{m l+}\right] p_{m}^{+}-2 \sum_{l<m} q_{l} q_{m}^{+} \lambda^{l m}-2 \sum_{l, m} q_{l}^{+} q_{m}^{+} \mathcal{P}^{l m}-\sum_{i<l<j} \vartheta_{l j}^{+} \vartheta_{i}^{+l} \lambda^{i j} \\
& -\sum_{l<n<m} \vartheta_{l m}^{+} \vartheta_{n}^{l} \lambda^{n m}+\sum_{n<l<m} \vartheta_{l m}^{+} \vartheta_{n}{ }^{m} \lambda^{+n l}-\sum_{n, l<m}\left(1+\delta_{l n}\right) \vartheta_{l m}^{+} \eta^{l+}{ }_{n} \mathcal{P}^{m n} \\
& +\sum_{n, l<m}\left(1+\delta_{m n}\right) \vartheta_{l m}^{+} \eta^{m}{ }_{n} \mathcal{P}^{+l n}+\frac{1}{2} \sum_{l<m, n \leq m} \eta_{n m}^{+} \eta_{l}^{n} \lambda^{l m} \\
& -\left[\frac{1}{2} \sum_{l, m}\left(1+\delta_{l m}\right) \eta^{m} \eta_{l m}^{+}+\sum_{l<m} \vartheta_{l m} \eta^{+m}+\sum_{m<l} \vartheta_{m l}^{+} \eta^{+m}\right] \mathcal{P}^{l}+h . c .
\end{aligned}
$$

Here, $\tilde{T}_{0}, \Delta Q$ are independent of $q_{0}, p_{0}, \eta_{0}, \mathcal{P}_{0}$, and the relation $\tilde{T}_{0}^{2}=-L_{0}$ holds true. We also expand the state vector and gauge parameters in powers of the zero-mode ghosts, for $s=$ $0, \ldots, \sum_{o=1}^{k} n_{o}+k(k-1) / 2-1$ :

$$
\begin{aligned}
|\chi\rangle & =\sum_{l=0}^{\sum_{o=1}^{k} n_{o}+k(k-1) / 2-1} q_{0}^{l}\left(\left|\chi_{0}^{l}\right\rangle+\eta_{0}\left|\chi_{1}^{l}\right\rangle\right), \quad g h\left(\left|\chi_{m}^{l}\right\rangle\right)=-(m+l), m=0,1 \\
\left|\Lambda^{(s)}\right\rangle & =\sum_{l=0}^{\sum_{o=1}^{k} n_{o}+k(k-1) / 2-1-s} q_{0}^{k}\left(\left|\Lambda_{0}^{(s) l}\right\rangle+\eta_{0}\left|\Lambda_{1}^{(s) l}\right\rangle\right), \quad g h\left(\left|\Lambda_{m}^{(s) l}\right\rangle\right)=-(s+l+m+1) .
\end{aligned}
$$

Now, we can gauge away all the fields and gauge parameters by using the equations of motion (5.17) and the set of gauge transformations (5.20)-(5.21), except for two, $\left|\chi_{0}^{0}\right\rangle,\left|\chi_{0}^{1}\right\rangle$ for the fields and $\left|\Lambda_{0}^{(s) l}\right\rangle$, for $l=0,1$ and $s=0, \ldots, \sum_{o=1}^{k} n_{o}+k(k-1) / 2-1$, for the gauge parameters. To do so, we follow, in part, the procedure described in [54, 55], [59]. Namely, after the extraction of zero-mode ghosts from the BRST operator $Q$ (5.23), as well as from the state vector and the gauge parameter (5.26), (5.27), the gauge transformation for the fields $\left|\chi_{0}^{l}\right\rangle, l \geq 2$ has the form

$$
\delta\left|\chi_{0}^{l}\right\rangle=\Delta Q\left|\Lambda_{0}^{l}\right\rangle+\eta_{i} \eta_{i}^{+}\left|\Lambda_{1}^{l}\right\rangle+(l+1)\left(q_{i} \eta_{i}^{+}-\eta_{i} q_{i}^{+}\right)\left|\Lambda_{0}^{l+1}\right\rangle+\tilde{T}_{0}\left|\Lambda_{0}^{l-1}\right\rangle+\left|\Lambda_{1}^{l-2}\right\rangle,
$$

implying, by induction, that we can make all the fields $\left|\chi_{0}^{l}\right\rangle, l \geq 2$ equal to zero by using the gauge parameters $\left|\Lambda_{1}^{l-2}\right\rangle$. Then, considering the equations of motion for the powers $q_{0}^{l}, l \geq 3$ and taking into account that $\left|\chi_{0}^{l}\right\rangle=0, l \geq 2$, we can see that these equations contain the subsystem

$$
\left|\chi_{1}^{l-2}\right\rangle=\eta_{i} \eta_{i}^{+}\left|\chi_{1}^{l}\right\rangle, \quad l \geq 3
$$

which permits one to find, by induction, that all the fields $\left|\chi_{1}^{l}\right\rangle, l \geq 1$ are equal to zero. Finally, we examine the equations of motion for the power $q_{0}^{2}$ :

$$
\left|\chi_{1}^{0}\right\rangle=-\tilde{T}_{0}\left|\chi_{0}^{1}\right\rangle
$$

in order to express the vector $\left|\chi_{1}^{0}\right\rangle$ in terms of $\left|\chi_{0}^{1}\right\rangle$. Therefore, as in the mixed-symmetric case with two rows in YT [59], there remain only two independent fields: $\left|\chi_{0}^{l}\right\rangle, l=0,1$. The above analysis is valid, of course, for the field vectors with a definite value of spin, i.e., for $\left|\chi_{0}^{l}\right\rangle_{(n)_{k}}$, $l=0,1$. 
The first equation in (5.10), with the representation (5.23), the decomposition (5.26), together with the above analysis, imply (in view of the fact that the operators $Q, \tilde{T}_{0}, \sum_{l}\left(\eta_{l}^{+} \eta_{l}\right)$ commute with $\sigma^{i}$ ) that independent equations of motion for these vectors have the form

$$
\begin{aligned}
& \Delta Q\left|\chi_{0}^{0}\right\rangle_{(n)_{k}}+\frac{1}{2}\left\{\tilde{T}_{0}, \eta_{i}^{+} \eta_{i}\right\}\left|\chi_{0}^{1}\right\rangle_{(n)_{k}}=0, \\
& \tilde{T}_{0}\left|\chi_{0}^{0}\right\rangle_{(n)_{k}}+\Delta Q\left|\chi_{0}^{1}\right\rangle_{(n)_{k}}=0
\end{aligned}
$$

where $\{$,$\} is the anticommutator and the fields \left|\chi_{0}^{l}\right\rangle_{(n)_{k}}, l=0,1$ are assumed to obey the spin relations (5.16) for $(m)_{k}=(n)_{k}$.

The equations of motion (5.31), (5.32) are Lagrangian and can be deduced, in view of the invertibility of the operator $K$ (3.16), (4.8), from the following Lagrangian action for a fixed spin $(m)_{k}=(n)_{k}$ (defined, in the standard manner, up to an overall factor),

$$
\begin{aligned}
\mathcal{S}_{(n)_{k}}= & { }_{(n)_{k}}\left\langle\tilde{\chi}_{0}^{0}\left|K_{(n)_{k}} \tilde{T}_{0}\right| \chi_{0}^{0}\right\rangle_{(n)_{k}}+\frac{1}{2}{ }_{(n)_{k}}\left\langle\tilde{\chi}_{0}^{1}\left|K_{(n)_{k}}\left\{\tilde{T}_{0}, \eta_{i}^{+} \eta_{i}\right\}\right| \chi_{0}^{1}\right\rangle_{(n)_{k}}, \\
& +{ }_{(n)_{k}}\left\langle\tilde{\chi}_{0}^{0}\left|K_{(n)_{k}} \Delta Q\right| \chi_{0}^{1}\right\rangle_{(n)_{k}}+{ }_{(n)_{k}}\left\langle\tilde{\chi}_{0}^{1}\left|K_{(n)_{k}} \Delta Q\right| \chi_{0}^{0}\right\rangle_{(n)_{k}},
\end{aligned}
$$

where the standard odd scalar product for the creation and annihilation operators is assumed, with the measure $d^{d} x$ over the Minkowski space. The vectors (Dirac spinors) $\left|\chi_{0}^{0}\right\rangle_{(n)_{k}},\left|\chi_{0}^{1}\right\rangle_{(n)_{k}}$ (5.26) as the solution of the spin distribution relations (5.16) are the respective vectors $\left|\chi_{0}^{l}\right\rangle$ in (5.8) for massless $(m=0)$ and massive $(m \neq 0)$ HS fermionic field $\Psi_{\left(\mu^{1}\right)_{n_{1}}, \ldots,\left(\mu^{k}\right)_{n_{k}}}(x)$ with the ghost number $g h\left(\left|\chi_{0}^{l}\right\rangle_{(n)_{k}}\right)=-l$, whereas $K_{(n)_{k}}$ is obtained from $K$ (4.8) with the substitution: $h^{i} \rightarrow-\left(n_{i}+(d-4 i+\theta(m)) / 2\right)$.

The action (5.33) and the equations of motion (5.31), (5.32) are invariant with respect to gauge transformations, following from the tower of relations (5.20)-(5.21):

$$
\begin{aligned}
\delta\left|\chi_{0}^{0}\right\rangle_{(n)_{k}} & =\Delta Q\left|\Lambda_{0}^{0}\right\rangle_{(n)_{k}}+\frac{1}{2}\left\{\tilde{T}_{0}, \eta_{i}^{+} \eta_{i}\right\}\left|\Lambda_{0}^{1}\right\rangle_{(n)_{k}}, \\
\delta\left|\chi_{0}^{1}\right\rangle_{(n)_{k}} & =\tilde{T}_{0}\left|\Lambda_{0}^{0}\right\rangle_{(n)_{k}}+\Delta Q\left|\Lambda_{0}^{1}\right\rangle_{(n)_{k}},
\end{aligned}
$$

which are reducible, with the gauge parameters $\left|\Lambda_{0}^{(s) j}\right\rangle_{(n)_{k}}, j=0,1$, subject to the same conditions as those for $\left|\chi_{0}^{j}\right\rangle_{(n)_{k}}$ in (5.16),

$$
\begin{array}{ll}
\delta\left|\Lambda_{0}^{(s) 0}\right\rangle_{(n)_{k}}=\Delta Q\left|\Lambda_{0}^{(s+1) 0}\right\rangle_{(n)_{k}}+\frac{1}{2}\left\{\tilde{T}_{0}, \eta_{i}^{+} \eta_{i}\right\}\left|\Lambda_{0}^{(s+1) 1}\right\rangle_{(n)_{k},}, & \left|\Lambda_{0}^{(0) 0}\right\rangle=\left|\Lambda_{0}^{0}\right\rangle, \\
\delta\left|\Lambda_{0}^{(s) 1}\right\rangle_{(n)_{k}}=\tilde{T}_{0}\left|\Lambda_{0}^{(s+1) 0}\right\rangle_{(n)_{k}}+\Delta Q\left|\Lambda_{0}^{(s+1) 1}\right\rangle_{(n)_{k}}, & \left|\Lambda_{0}^{(0) 1}\right\rangle=\left|\Lambda_{0}^{1}\right\rangle,
\end{array}
$$

and with a finite number of reducibility stage (the same as for the case of $q_{0}$-dependent vectors $|\chi\rangle,\left|\Lambda^{(s)}\right\rangle$ in (5.17), (5.20)-(5.21) $)$ to be equal to $s=\sum_{o=1}^{k} n_{o}+k(k-1) / 2-1$.

A simultaneous construction of Lagrangian actions describing the propagation of all massless (massive) fermionic fields with two rows of the Young tableaux in the Minkowski space is similar to the case of totally symmetric spin-tensors in the flat space [55]. Note that a necessary condition for solving this problem is to replace in $Q^{\prime}, Q, K$ the parameters $-h^{i}$ by the operators $\sigma^{i}$ in an appropriate manner and discard the condition (5.16) for the fields and gauge parameters.

In what follows, we consider some examples of the Lagrangian formulation procedure.

\section{$6 \quad$ Examples}

Here, we realize the general prescriptions of our Lagrangian formulation in the case of mixedsymmetry fermionic fields of the lowest values of rows and spins. 


\subsection{Spin $\left(n_{1}+\frac{1}{2}, n_{2}+\frac{1}{2}\right)$ Mixed-symmetric Field}

As the first example, let us examine the mixed-symmetric spin-tensor with two sets of indices corresponding to spin- $\left(n_{1}+\frac{1}{2}, n_{2}+\frac{1}{2}\right)$. In this case, we expect that our result will coincide with the one in the massless case [59], and will be a new one for massive12, where the respective mixedsymmetric massless and massive spin-tensors, subject to $Y\left(s_{1}, s_{2}\right)$, were considered. According to our procedure, we have $\left(m_{1}, m_{2}\right)=\left(n_{1}, n_{2}\right), m_{i}=0$, for $i=3, \ldots, k$. We can show, given $m_{i}=0$, that in (2.6) and (5.8) all the components related to the rows $i \geq 3$ in the Young tableaux must vanish, i.e.,

$$
\begin{aligned}
n_{l}^{\prime} & =n_{a l}=n_{b l}=n_{l}^{0}=n_{1 j}=n_{2 m}=p_{1 s}=p_{2 t}=n_{f i}=n_{p j}=n_{f 1 j}=n_{f 2 m}=n_{p 1 j}=n_{p 2 o} \\
& =n_{f 1 s}=n_{f 2 t}=n_{\lambda 1 s}=n_{\lambda 2 t}=n_{s_{m}}=0, \text { for } l, j, m, s, t, i, o>2 .
\end{aligned}
$$

As a result, the only surviving state vectors $\left|\chi_{0}^{l}\right\rangle, l=0,1$ from Eqs. (5.26) are reduced to

$$
\begin{aligned}
& \left|\chi_{0}^{l}\right\rangle=\sum_{n} \prod_{c}^{2}\left(f_{c}^{+}\right)^{n_{c}^{0}} \prod_{a}^{2}\left(b_{a}^{+}\right)^{n_{a}^{\prime}} \prod_{i \leq j}^{2}\left(b_{i j}^{+}\right)^{n_{i j}}\left(d_{12}^{+}\right)^{p_{12}} \prod_{e, g}^{2}\left(q_{e}^{+}\right)^{n_{a e}}\left(p_{g}^{+}\right)^{n_{b g}} \\
& \times \prod_{i, j, l \leq m, n \leq o}^{2}\left(\eta_{i}^{+}\right)^{n_{f i}}\left(\mathcal{P}_{j}^{+}\right)^{n_{p j}}\left(\eta_{l m}^{+}\right)^{n_{f l m}}\left(\mathcal{P}_{n o}^{+}\right)^{n_{p n o}}\left(\vartheta_{12}^{+}\right)^{n_{f 12}}\left(\lambda_{12}^{+}\right)^{n_{\lambda 12}} \\
& \times \mid \Psi\left(a_{1}^{+}, a_{2}^{+}\right) \begin{array}{l}
l 0_{f 0} ;(n)_{a e}(n)_{b g}(n)_{f i}(n)_{p j}(n)_{f l m}(n)_{p n o} n_{f 12} n_{\lambda 12} \\
\left(n^{0}\right)_{c} ;\left(n^{\prime}\right)_{l}(n)_{i j} p_{12}
\end{array}, \\
& \left|\Psi\left(a_{1}^{+}, a_{2}^{+}\right)_{\left(n^{0}\right)_{c} ;\left(n^{\prime}\right)_{l}(n)_{i j} p_{12}}^{l 0_{f 0} ; . n_{\lambda 1}}\right\rangle=\sum_{p_{1}=0}^{\infty} \sum_{p_{2}=0}^{p_{1}} \Psi_{\left(n^{0}\right)_{c} ;\left(n^{\prime}\right)_{l}(n)_{i j} p_{12}\left(\mu^{1}\right)_{p_{1}},\left(\mu^{2}\right)_{p_{2}},(0)_{n_{3} \ldots,} \ldots(0)_{n_{k}}}^{l 0_{f 0} \ldots n_{n_{12}}}(x) \\
& \times \prod_{l_{1}=1}^{p_{1}} a_{1}^{+\mu_{l_{1}}^{1}} \prod_{l_{2}=1}^{p_{2}} a_{2}^{+\mu_{l_{2}}^{2}}|0\rangle
\end{aligned}
$$

These vectors correspond to those in the massless case, $\left(n^{\prime}\right)_{l}=(0)_{l}$, in [59], and are new in massive cases. The representation (6.2), (6.3) is valid for the sequence of gauge parameters $\left|\Lambda_{0}^{(s) l}\right\rangle_{(n)_{2}}$ from the tower of reducible gauge transformations (5.34)-(5.37) with the maximal value of reducibility stage $s_{\max }=n_{1}+n_{2}$. The operator $C^{r s}\left(d^{+}, d\right)$ in (3.10) has the same form as in the integer-spin case [31], and it is only $C^{12}\left(d^{+}, d\right)$ that has a non-vanishing value:

$$
C^{12}\left(d^{+}, d\right) \equiv\left(h^{1}-h^{2}-d_{12}^{+} d_{12}\right) d_{12},
$$

so that the expression for the $\operatorname{ssp}(1 \mid 4)$ algebra auxiliary representation can be easily deduced from Eqs.(3.1)-(3.9). Then, one can easily show that equations (5.31), (5.32), relations (5.34)-(5.37), and action (5.33) with $\left|\chi_{0}^{l}\right\rangle, l=0,1$, as in (6.2), (6.3), reproduce the same relations as those in [59] for the massless case, and new ones for the massive case.

\subsection{Spin $\left(n_{1}+\frac{1}{2}, n_{2}+\frac{1}{2}, n_{3}+\frac{1}{2}\right)$ General mixed-symmetric Field}

In this subsection, we examine a new, yet unknown, Lagrangian formulation for the mixed-symmetric HS field $\Psi_{\left(\mu^{1}\right)_{n_{1}},\left(\mu^{2}\right)_{n_{2}},\left(\mu^{3}\right)_{n_{3}}}$ with three families of symmetric indices subject to $Y\left(n_{1}, n_{2}, n_{3}\right)$. The values of $\operatorname{spin}\left(n_{1}+\frac{1}{2}, n_{2}+\frac{1}{2}, n_{3}+\frac{1}{2}\right)$ for $n_{1} \geq n_{2} \geq n_{3}$ can be composed from the set of coefficients $\left(l, n_{c}^{0}, n_{a e}, n_{b g}, n_{l^{\prime}}^{\prime}, n_{i j}, p_{r s}, n_{f 0}, n_{f i}, n_{p j}, n_{f l m}, n_{p n o}, n_{f r s}, n_{\lambda t u}, p_{i}\right)$, for $l=0,1$ and

\footnotetext{
${ }^{12}$ In [59], a Lagrangian formulation for massive fermionic fields subject to a Young tableaux $Y\left(n_{1}+\frac{1}{2}, n_{2}+\frac{1}{2}\right)$ on $\mathbb{R}^{1, d-1}$ was realized by dimensional reduction from a massless theory in a $(d+1)$-dimensional Minkowski space at the stage of a component formulation only, see Eqs.(5.34)-(5.36) therein.
} 
$c, e, g, l, i, j, r, s, l^{\prime}, m, n, o, t, u=1,2,3, i \leq j, r<s, l^{\prime} \leq m, n \leq o, t<u$, in (5.8), (15.26) and $(2.6){ }^{13}$ to be restricted for all vectors $\left|\chi_{0}^{l}\right\rangle_{(n)_{3}},\left|\Lambda_{0}^{(s) l}\right\rangle_{(n)_{3}} l=0,1 ; s=0, \ldots, \sum_{o=1}^{3} n_{o}+2$, in view of the spectral problem solution (5.16) and the general decomposition (5.18) for spin [for the field subject to $Y\left(n_{1}, \ldots, n_{k}\right)$ ] by the formulae

$$
\begin{aligned}
n_{i}= & p_{i}+\Theta(m) n_{i}^{\prime}+n_{a i}+n_{b i}+n_{i}^{0}+\sum_{j=1}\left(1+\delta_{i j}\right)\left(n_{i j}+n_{f i j}+n_{p i j}\right)+n_{f i}+n_{p i} \\
& +\sum_{r<i}\left(p_{r i}+n_{f r i}+n_{\lambda r i}\right)-\sum_{r>i}\left(p_{i r}+n_{f i r}+n_{\lambda i r}\right), i=1,2,3 .
\end{aligned}
$$

In addition to restrictions (6.5), the subset of "ghost" numbers $\left(l, n_{f i}, n_{a e}, n_{b g}, n_{p j}, n_{f l m}, n_{p n o}\right.$, $\left.n_{f r s}, n_{\lambda t u}\right)$ in (5.8), without the $\eta_{0}$-number, should also obey such relations as the general ones (5.19) and (2.6) for fixed values of $n_{i}$; it satisfies the following equations for the field vectors $\left|\chi_{0}^{l}\right\rangle_{(n)_{3}}$, and for the set of gauge parameters $\left|\Lambda_{0}^{(s) l}\right\rangle_{(n)_{3}}$, for $l=n_{b 0}=0,1, s=0 \ldots, \sum_{o=1}^{3} n_{o}+2$ :

$$
\begin{aligned}
\left|\chi_{0}^{l}\right\rangle_{(n)_{3}}: \sum_{i}\left(n_{f i}-n_{p i}+n_{a i}-n_{b i}\right)+\sum_{i \leq j}\left(n_{f i j}-n_{p i j}\right)+\sum_{r<s}\left(n_{f r s}-n_{\lambda r s}\right) & =-l \\
\left|\Lambda_{0}^{(s) l}\right\rangle_{(n)_{3}}: & \sum_{i}\left(n_{f i}-n_{p i}+n_{a i}-n_{b i}\right)+\sum_{i \leq j}\left(n_{f i j}-n_{p i j}\right)+\sum_{r<s}\left(n_{f r s}-n_{\lambda r s}\right)=-l-s-1(6 .
\end{aligned}
$$

which follows from the ghost number distributions (5.10)-(5.13). Note that the above $2(k+$ $3+\sum_{o=1}^{3} n_{o}$ ) relations (6.5)-(6.7) express both the fact of the general homogeneity of the vectors $\left|\chi_{0}^{l}\right\rangle_{(n)_{3}},\left|\Lambda_{0}^{(s) l}\right\rangle_{(n)_{3}}$ with respect to the spin and ghost number distributions, and completely describe the internal structure of these vectors in the powers of oscillators in $\mathcal{H}_{\text {tot }}$.

The underlying part $\Delta Q$ (5.25) of the BRST operator $Q$ (5.23) for 7 odd $\left(T_{0}, T_{i}, T_{i}^{+}\right)$and 25 even constraints $\left(L_{0}, L_{i}, L_{i j}, T_{r s}, L_{i}^{+}, L_{i j}^{+}, T_{r s}^{+}\right)$reduces to the form

$$
\begin{aligned}
\Delta Q= & q_{i}^{+} T^{i}+\eta_{i}^{+} L^{i}+\sum_{l \leq m} \eta_{l m}^{+} L^{l m}+\sum_{l<m} \vartheta_{l m}^{+} T^{l m}+\left[\frac{1}{2} \sum_{l, m}\left(1+\delta_{l m}\right) \eta^{l m} q_{l}^{+}-\sum_{l<m} q_{l} \vartheta^{l m}\right. \\
& \left.-\sum_{m<l} q_{l} \vartheta^{m l+}\right] p_{m}^{+}-2 \sum_{l<m} q_{l} q_{m}^{+} \lambda^{l m}-2 \sum_{l, m} q_{l}^{+} q_{m}^{+} \mathcal{P}^{l m}-\vartheta_{23}^{+}\left(\vartheta_{12}^{+} \lambda^{13}-\vartheta_{13} \lambda_{12}^{+}\right) \\
& -\vartheta_{13}^{+} \vartheta_{12} \lambda^{23}-\sum_{n, l<m}\left(1+\delta_{l n}\right) \vartheta_{l m}^{+} \eta^{l+}{ }_{n} \mathcal{P}^{m n}+\sum_{n, l<m}\left(1+\delta_{m n}\right) \vartheta_{l m}^{+} \eta^{m}{ }_{n} \mathcal{P}^{+l n} \\
& +\frac{1}{2} \sum_{l<m, n \leq m} \eta_{n m}^{+} \eta_{l}^{n} \lambda^{l m}-\left[\frac{1}{2} \sum_{l, m}\left(1+\delta_{l m}\right) \eta^{m} \eta_{l m}^{+}+\sum_{l<m} \vartheta_{l m} \eta^{+m}+\sum_{m<l} \vartheta_{m l}^{+} \eta^{+m}\right] \mathcal{P}^{l}+h . c .,
\end{aligned}
$$

whereas the extended (by the ghosts $\tilde{T}_{0}$ ) constraint reads exactly as in (15.24), however, for $k=3$. The BRST operator $Q$ determined by decomposition (5.23) is nilpotent after the substitution $h_{i} \rightarrow-\left(p_{i}+\frac{d-4 i+\theta(m)}{2}\right)$, for $i=1,2,3$, when restricted to the Hilbert subspace in $\mathcal{H}_{t o t}$, formed by the vectors $\left|\chi_{0}^{l}\right\rangle_{(n)_{3}}($ (5.26) $)$, and $\left|\Lambda_{0}^{(s) l}\right\rangle_{(n)_{3}}$ (5.27), proper for the spin operator $\left(\sigma^{1}, \sigma^{2}, \sigma^{3}\right)$ (5.2).

The explicit form of the additional parts to the second-class constraints $o_{a}^{\prime}$ is determined by relations (3.1)-(3.9), however, for $k=3$ rows in YT, so that the operators $t_{m}^{\prime+}, t_{l m}^{\prime+}$, for $l, m=1,2,3$;

\footnotetext{
${ }^{13}$ We replace the indices $n_{i}$ in (2.6) for the vector in the initial Fock space $\mathcal{H}$ by $p_{i}$ due to the use of $n_{i}$ for the value of generalized spin of the basic HS spin-tensor $\Psi_{\left(\mu^{1}\right)_{n_{1}},\left(\mu^{2}\right)_{n_{2}},\left(\mu^{3}\right)_{n_{3}}}$.
} 
$l<m$ in (3.1), (3.2) are written as follows:

$$
\begin{aligned}
& t_{1}^{\prime+}=f_{1}^{+}+2 b_{11}^{+} f_{1}, \\
& t_{2}^{\prime+}=f_{2}^{+}+2 b_{22}^{+} f_{2}+4 b_{12}^{+} f_{1}, \\
& t_{3}^{\prime+}=f_{3}^{+}+2 b_{33}^{+} f_{3}+4\left(b_{13}^{+} f_{1}+b_{23}^{+} f_{2}\right), \\
& t_{12}^{\prime+}=d_{12}^{+}-\sum_{n=1}^{3}\left(1+\delta_{1 n}\right) b_{n 2}^{+} b_{1 n}-\left(f_{2}^{+}+2 b_{22}^{+} f_{2}\right) f_{1}, \\
& t_{13}^{\prime+}=d_{13}^{+}-\sum_{n=1}^{3}\left(1+\delta_{1 n}\right) b_{n 3}^{+} b_{1 n}-\left[4 b_{23}^{+} f_{2}+f_{3}^{+}+2 b_{33}^{+} f_{3}\right] f_{1}, \\
& t_{23}^{\prime+}=d_{23}^{+}-d_{12} d_{13}^{+}-\sum_{n=1}^{3}\left(1+\delta_{n 2}\right) b_{n 3}^{+} b_{2 n}-\left(f_{3}^{+}+2 b_{33}^{+} f_{3}\right) f_{2},
\end{aligned}
$$

where one must take into account expressions for the operators $C^{12}\left(d^{+}, d\right), C^{13}\left(d^{+}, d\right), C^{23}\left(d^{+}, d\right)$ (defined for the first time in [31] for a general bosonic field with three groups of symmetric indices):

$$
\begin{aligned}
& C^{12}\left(d^{+}, d\right) \equiv\left(h^{1}-h^{2}-d_{12}^{+} d_{12}-d_{13}^{+} d_{13}-d_{23}^{+} d_{23}\right) d_{12}+\left\{d_{23}^{+}-d_{13}^{+} d_{12}\right\} d_{13}, \\
& C^{13}\left(d^{+}, d\right) \equiv\left(h^{1}-h^{3}-d_{13}^{+} d_{13}+d_{23}^{+} d_{23}\right) d_{13}, \\
& C^{23}\left(d^{+}, d\right) \equiv\left(h^{2}-h^{3}-d_{23}^{+} d_{23}\right) d_{23} .
\end{aligned}
$$

Second, Eqs. (6.15)-(6.17) permit one to present the expressions for the odd "gamma-traceless" elements $t_{i}^{\prime}$ as follows:

$$
\begin{aligned}
t_{1}^{\prime}= & 2 \sum_{n=2}^{3}\left\{d_{1 n}^{+}-\sum_{m=1}^{3}\left(1+\delta_{1 m}\right) b_{n m}^{+} b_{1 m}\right\} f_{n} \\
& -2\left(\sum_{m=1}^{3}\left(1+\delta_{1 m}\right) b_{1 m}^{+} b_{1 m}-d_{12}^{+} d_{12}-d_{13}^{+} d_{13}+h^{1}\right) f_{1} \\
& +\sum_{n=1}^{3}\left(1+\delta_{1 n}\right)\left\{2 \sum_{m=n+1}^{3} b_{n m}^{+} f_{m}-\frac{1}{2}\left(f_{n}^{+}-2 b_{n n}^{+} f_{n}\right)\right\} b_{1 n} \\
t_{2}^{\prime}= & -2\left\{-C^{12}\left(d^{+}, d\right)+\sum_{m=1}^{3}\left(1+\delta_{m 2}\right) b_{1 m}^{+} b_{2 m}+f_{1}^{+} f_{2}\right\} f_{1} \\
& +2\left\{d_{23}^{+}-d_{13}^{+} d_{12}-\sum_{m=1}^{3}\left(1+\delta_{m 2}\right) b_{3 m}^{+} b_{2 m}\right\} f_{3} \\
& -2\left(\sum_{l=1}^{3}\left(1+\delta_{2 l}\right) b_{2 l}^{+} b_{2 l}-d_{23}^{+} d_{23}+d_{12}^{+} d_{12}+h^{2}\right) f_{2} \\
& +\sum_{n=1}^{3}\left(1+\delta_{n 2}\right)\left\{2 \sum_{m=n+1}^{3} b_{n m}^{+} f_{m}-\frac{1}{2}\left(f_{n}^{+}-2 b_{n n}^{+} f_{n}\right)\right\} b_{n 2} \\
t_{3}^{\prime}= & -2\left\{-C^{13}\left(d^{+}, d\right)-C^{23}\left(d^{+}, d\right) d_{12}+\sum_{m=1}^{3}\left(1+\delta_{m 3}\right) b_{1 m}^{+} b_{m 3}-\left[4 b_{12}^{+} f_{2}-f_{1}^{+}\right] f_{3}\right\} f_{1}(6.2) \\
& -2\left\{d_{12}^{+} d_{13}-C^{23}\left(d^{+}, d\right)+\sum_{m=1}^{3}\left(1+\delta_{m 3}\right) b_{m 2}^{+} b_{m 3}+f_{2}^{+} f_{3}\right\} f_{2} \\
& -2\left(\sum_{l=1}^{3}\left(1+\delta_{3 l}\right) b_{l 3}^{+} b_{l 3}+d_{13}^{+} d_{13}+d_{23}^{+} d_{23}+h^{3}\right) f_{3} \\
& +\sum_{n=1}^{3}\left(1+\delta_{n 3}\right)\left\{2 \sum_{m=n+1}^{3} b_{n m}^{+} f_{m}-\frac{1}{2}\left(f_{n}^{+}-2 b_{n n}^{+} f_{n}\right)\right\} b_{n 3} .
\end{aligned}
$$


In turn, for the even "traceless" elements $l_{l l}^{\prime}$ we have

$$
\begin{aligned}
l_{11}^{\prime}= & -\left[2\left\{d_{12}^{+}-\sum_{n^{\prime}=1}^{3}\left(1+\delta_{n^{\prime} 1}\right) b_{n^{\prime} 2}^{+} b_{n^{\prime} 1}\right\} f_{2}+2\left\{d_{13}^{+}-\sum_{n^{\prime}=1}^{3}\left(1+\delta_{n^{\prime} 1}\right) b_{n^{\prime} 3}^{+} b_{n^{\prime} 1}\right\} f_{3}\right. \\
& \left.-\sum_{n=1}^{3}\left(1+\delta_{1 n}\right)\left\{-2 \sum_{m=n+1}^{3} b_{n m}^{+} f_{m}+\frac{1}{2}\left[f_{n}^{+}-\left(1-\delta_{1 n}\right) 2 b_{n n}^{+} f_{n}\right]\right\} b_{1 n}\right] f_{1} \\
& +\frac{1}{4} \sum_{n=2}^{3}\left[b_{n n}^{+} b_{1 n}-2 d_{1 n}^{+}+2 b_{n 3}^{+} b_{13}\right] b_{1 n}+\left(\sum_{n=1}^{3} b_{1 n}^{+} b_{1 n}-d_{12}^{+} d_{12}-d_{13}^{+} d_{13}+h^{1}\right) b_{11}, \\
l_{22}^{\prime}= & -\left[2\left\{d_{23}^{+}-d_{13}^{+} d_{12}-\sum_{n^{\prime}=1}^{3}\left(1+\delta_{n^{\prime} 2}\right) b_{n^{\prime} 3}^{+} b_{n^{\prime} 2}\right\} f_{3}\right. \\
& \left.-\sum_{n=1}^{3}\left(1+\delta_{n 2}\right)\left\{-2 \sum_{m=n+1}^{3} b_{n m}^{+} f_{m}+\frac{1}{2}\left[f_{n}^{+}-\left(1-\delta_{n 2}\right) 2 b_{n n}^{+} f_{n}\right]\right\} b_{2 n}\right] f_{2} \\
& +\frac{1}{4}\left(b_{11}^{+} b_{12}^{2}+b_{33}^{+} b_{23}^{2}\right)+\frac{1}{2}\left[2 b_{12}^{+} b_{22}+b_{13}^{+} b_{23}-C^{12}\left(d^{+}, d\right)\right] b_{12} \\
& +\left(b_{22}^{+} b_{22}+b_{23}^{+} b_{23}-d_{23}^{+} d_{23}+d_{12}^{+} d_{12}+h^{2}\right) b_{22}-\frac{1}{2}\left[d_{23}^{+}-d_{13}^{+} d_{12}\right] b_{23}, \\
l_{33}^{\prime}= & \sum_{n=1}^{3}\left(1+\delta_{n 3}\right)\left\{-2 \sum_{m=n+1}^{3} b_{n m}^{+} f_{m}+\frac{1}{2}\left[f_{n}^{+}-\left(1-\delta_{n 3}\right) 2 b_{n n}^{+} f_{n}\right]\right\} b_{3 n} f_{3} \\
& \frac{1}{4}\left(b_{11}^{+} b_{13}^{2}+b_{22}^{+} b_{23}^{2}\right)+\frac{1}{2}\left[d_{12}^{+} d_{13}+2 b_{23}^{+} b_{33}-C^{23}\left(d^{+}, d\right)\right] b_{23} \\
& +\frac{1}{2}\left[b_{12}^{+} b_{23}+2 b_{13}^{+} b_{33}-C^{13}\left(d^{+}, d\right)-C^{23}\left(d^{+}, d\right) d_{12}\right] b_{13} \\
& +\left(b_{33}^{+} b_{33}+d_{13}^{+} d_{13}+d_{23}^{+} d_{23}+h^{3}\right) b_{33},
\end{aligned}
$$

and for the same elements $l_{l m}^{\prime}$, but for $l<m$,

$$
\begin{aligned}
l_{12}^{\prime}= & -\left[\frac{1}{2} \sum_{n=1}^{3}\left(1+\delta_{n 2}\right)\left\{2 \sum_{n^{\prime}=n+1}^{3} b_{n n^{\prime}}^{+} f_{n^{\prime}}-\frac{1}{2}\left[f_{n}^{+}-\left(1-\delta_{n 1}\right) 2 b_{n n}^{+} f_{n}\right]\right\} b_{n 2}\right. \\
& -\left(\sum_{n=1}^{3}\left(1+\delta_{n 2}\right) b_{2 n}^{+} b_{2 n}-d_{23}^{+} d_{23}+d_{12}^{+} d_{12}+h^{2}\right) f_{2} \\
& \left.+\left\{d_{23}^{+}-d_{13}^{+} d_{12}-\sum_{n^{\prime}=1}^{3}\left(1+\delta_{n^{\prime} 2}\right) b_{n^{\prime} 3}^{+} b_{2 n^{\prime}}\right\} f_{3}\right] f_{1} \\
& -\left[\frac{1}{2} \sum_{n=1}^{3}\left(1+\delta_{n 1}\right)\left\{2 \sum_{n^{\prime}=n+1}^{3} b_{n n^{\prime}}^{+} f_{n^{\prime}}-\frac{1}{2}\left[f_{n}^{+}-\left(1-\delta_{n 2}\right) 2 b_{n n}^{+} f_{n}\right]\right\} b_{n 1}\right. \\
& \left.+\left\{d_{13}^{+}-\sum_{n^{\prime}=1}^{3}\left(1+\delta_{n^{\prime} 1}\right) b_{3 n^{\prime}}^{+} b_{1 n^{\prime}}\right\} f_{3}\right] f_{2} \\
& \frac{1}{4}\left(\sum_{n=2}^{3}\left[b_{1 n}^{+} b_{1 n}+\left(1+\delta_{n 2}\right) b_{2 n}^{+} b_{2 n}\right]-d_{13}^{+} d_{13}-d_{23}^{+} d_{23}+h^{1}+h^{2}\right) b_{12}
\end{aligned}
$$




$$
\begin{aligned}
& -\frac{1}{2}\left[C^{12}\left(d^{+}, d\right)-\sum_{n=1}^{3}\left(1+\delta_{n 2}\right) b_{1 n^{+}}^{+} b_{n 2}\right] b_{11}-\frac{1}{4}\left[2 d_{12}^{+} b_{22}+d_{13}^{+} b_{23}\right] \\
& -\frac{1}{4}\left[d_{23}^{+}-d_{13}^{+} d_{12}-\sum_{n=2}^{3}\left(1+\delta_{n 2}\right) b_{n 3}^{+} b_{2 n}\right] b_{13}, \\
l_{13}^{\prime}= & -\left[\left\{-d_{12}^{+} d_{13}+C^{23}\left(d^{+}, d\right)-\sum_{n^{\prime}=1}^{3}\left(1+\delta_{n^{\prime} 3}\right) b_{n^{\prime} 2}^{+} b_{n^{\prime} 3}-f_{2}^{+} f_{3}\right\} f_{2}\right. \\
& -\left(\sum_{n=1}^{3}\left(1+\delta_{n 3}\right) b_{n 3}^{+} b_{n 3}+d_{13}^{+} d_{13}+d_{23}^{+} d_{23}+h^{3}\right) f_{3} \\
+ & \left.\frac{1}{2} \sum_{n=1}^{3}\left(1+\delta_{n 3}\right)\left\{2 \sum_{n^{\prime}=n+1}^{3} b_{n n^{\prime}}^{+} f_{n^{\prime}}-\frac{1}{2}\left[f_{n}^{+}-\left(1-\delta_{1 n}\right) 2 b_{n n}^{+} f_{n}\right]\right\} b_{n 3}\right] f_{1} \\
& -\frac{1}{2} \sum_{n=1}^{3}\left(1+\delta_{1 n}\right)\left\{2 \sum_{n^{\prime}=n+1}^{k} b_{n n^{\prime}}^{+} f_{n^{\prime}}-\frac{1}{2}\left[f_{n}^{+}-\left(1-\delta_{n 3}\right) 2 b_{n n}^{+} f_{n}\right]\right\} b_{1 n} f_{3} \\
& \frac{1}{4}\left(b_{13}^{+} b_{13}+\sum_{n=2}^{3}\left(1+\delta_{n 3}\right) b_{n 3}^{+} b_{n 3}-d_{12}^{+} d_{12}+d_{23}^{+} d_{23}+h^{1}+h^{3}\right) b_{13} \\
+ & \frac{1}{4}\left[\sum_{n=2}^{3}\left(1+\delta_{n 3}\right) b_{2 n}^{+} b_{n 3}+d_{12}^{+} d_{13}-C^{23}\left(d^{+}, d\right)\right] b_{12}-\frac{1}{4}\left[d_{12}^{+} b_{23}+2 d_{13}^{+} b_{33}\right] \\
+ & \frac{1}{2}\left[\sum_{n=1}^{3}\left(1+\delta_{n 3}\right) b_{1 n}^{+} b_{n 3}-C^{13}\left(d^{+}, d\right)-C^{23}\left(d^{+}, d\right) d_{12}\right] b_{11}, \\
+ & -\left[-\left\{\sum_{n=1}^{3}\left(1+\delta_{n 3}\right) b_{n 3}^{+} b_{n 3}+d_{13}^{+} d_{13}+d_{23}^{+} d_{23}+h^{3}\right\} f_{3}\right. \\
& +\frac{1}{4}\left[C^{12}\left(d^{+}, d\right)-\sum_{n=2}^{3}\left(1+\delta_{n 2}\right) b_{1 n}^{+} b_{2 n}\right] b_{13}-\frac{1}{2}\left[d_{23}^{+}-d_{13}^{+} d_{12}\right] b_{33} . \\
+ & \left.\frac{1}{2} \sum_{n=1}^{3}\left(1+\delta_{n 3}\right)\left\{2 \sum_{n^{\prime}=n+1}^{3} b_{n n^{\prime}}^{+} f_{n^{\prime}}-\frac{1}{2}\left[f_{n}^{+}-\left(1-\delta_{n 2}\right) 2 b_{n n}^{+} f_{n}\right]\right\} b_{n 3}\right] f_{2} \\
& \left.-\left[\frac{1}{2} \sum_{n=1}^{3}\left(1+\delta_{n 2}\right)\left\{2 \sum_{n^{\prime}=n+1}^{3} b_{n n^{\prime}}^{+} f_{n^{\prime}}-\frac{1}{2}\left[f_{n}^{+}-\left(1-\delta_{n 3}\right) 2 b_{n n}^{+} f_{n}\right]\right\} b_{n 2}\right\}\right] f_{3} \\
& \frac{1}{4}\left[\sum_{n=1}^{3}\left(1+\delta_{n 3}\right) b_{2 n}^{+} b_{n 3}-C^{13}\left(d^{+}, d\right)-C^{23}\left(d^{+}, d\right) d_{12}\right] b_{12} \\
+ & \frac{1}{2}\left[\sum_{n=2}^{3}\left(1+\delta_{n 3}\right) b_{2 n}^{+} b_{n 3}+d_{12}^{+} d_{13}-C^{23}\left(d^{+}, d\right)\right] b_{22} \\
+ & \left(b_{23}^{+} b_{23}+2 b_{33}^{+} b_{33}+d_{12}^{+} d_{12}+d_{13}^{+} d_{13}+h^{2}+h^{3}\right) b_{23} \\
= &
\end{aligned}
$$


Finally, for "mixed-symmetry" elements $t_{l m}^{\prime}$, we have

$$
\begin{aligned}
t_{12}^{\prime}= & C^{12}\left(d^{+}, d\right)-\sum_{n=1}^{3}\left(1+\delta_{n 2}\right) b_{n 1}^{+} b_{n 2}+\left(2 b_{11}^{+} f_{1}-f_{1}^{+}\right) f_{2}, \\
t_{13}^{\prime}= & C^{13}\left(d^{+}, d\right)+C^{23}\left(d^{+}, d\right) d_{12}-\sum_{n=1}^{3}\left(1+\delta_{n 3}\right) b_{n 1}^{+} b_{n 3} \\
& +\left[4 b_{12}^{+} f_{2}+2 b_{11}^{+} f_{1}-f_{1}^{+}\right] f_{3}, \\
t_{23}^{\prime}= & -d_{12}^{+} d_{13}+C^{23}\left(d^{+}, d\right)-\sum_{n=1}^{3}\left(1+\delta_{n 3}\right) b_{n 2}^{+} b_{n 3}+\left(2 b_{22}^{+} f_{2}-f_{2}^{+}\right) f_{3} .
\end{aligned}
$$

Relations (6.9)-(6.29) together with Eqs. (3.1), (3.3) for $l_{i j}^{\prime+}$ and the particle number operators $g_{0}^{\prime i}$ for the value of $k=3$, compose the scalar oscillator realization of the $\operatorname{ssp}(1 \mid 6)$ superalgebra over the Heisenberg-Weyl superalgebra $A_{3 \mid 9}$ with 6 odd and 18 even independent operators $\left(f_{i}^{+}, f_{i}\right)$, $\left(b_{i j}^{+}, b_{i j}, d_{r s}^{+}, d_{r s}\right)$, for $i \leq j, r<m$. The above expressions for the vanishing of all operators $t_{i}^{\prime+}, t_{i}^{\prime}, t_{r s}^{\prime+}, t_{r s}^{\prime}, l_{l m}^{+}, l_{l m}^{\prime}, g_{0}^{\prime i}$ and $f_{i}^{+}, f_{i}, b_{l m}^{+}, b_{l m}, d_{r s}^{+}, d_{r s}$, for $i, l, m, s=3$ are reduced to the oscillator realization of the $\operatorname{ssp}(1 \mid 4)$ superalgebra over the Heisenberg-Weyl superalgebra $A_{2 \mid 6}$, obtained for a massless mixed symmetry fermionic HS field in Minkowski space with 2 rows in the YT [59].

It is now easy to present the Lagrangian equations of motion (5.31), (5.32), the set of reducible Abelian gauge transformations (5.34)-(5.37), and the unconstrained action $\mathcal{S}_{(n)_{3}}$ (5.33), which have the final (respective) form, for the HS field of spin $\left(n_{1}+\frac{1}{2}, n_{2}+\frac{1}{2}, n_{3}+\frac{1}{2}\right)$, with $s=$ $0, \ldots, n_{1}+n_{2}+n_{3}+2$,

$$
\begin{aligned}
& \Delta Q\left|\chi_{0}^{0}\right\rangle_{(n)_{3}}+\frac{1}{2}\left\{\tilde{T}_{0}, \eta_{i}^{+} \eta_{i}\right\}\left|\chi_{0}^{1}\right\rangle_{(n)_{3}}=0, \quad \tilde{T}_{0}\left|\chi_{0}^{0}\right\rangle_{(n)_{k}}+\Delta Q\left|\chi_{0}^{1}\right\rangle_{(n)_{k}}=0 \\
& \delta\left|\chi_{0}^{0}\right\rangle_{(n)_{3}}=\Delta Q\left|\Lambda_{0}^{0}\right\rangle_{(n)_{3}}+\frac{1}{2}\left\{\tilde{T}_{0}, \eta_{i}^{+} \eta_{i}\right\}\left|\Lambda_{0}^{1}\right\rangle_{(n)_{3}}, \quad \delta\left|\chi_{0}^{1}\right\rangle_{(n)_{3}}=\tilde{T}_{0}\left|\Lambda_{0}^{0}\right\rangle_{(n)_{3}}+\Delta Q\left|\Lambda_{0}^{1}\right\rangle_{(n)_{3}} \\
& \delta\left|\Lambda_{0}^{(s) 0}\right\rangle_{(n)_{3}}=\Delta Q\left|\Lambda^{(s+1) 0}\right\rangle_{(n)_{3}}+\frac{1}{2}\left\{\tilde{T}_{0}, \eta_{i}^{+} \eta_{i}\right\}\left|\Lambda^{(s+1) 1}\right\rangle_{\left.{ }_{(n)}\right)_{3}} \\
& \delta\left|\Lambda_{0}^{(s) 1}\right\rangle_{(n)_{3}}=\tilde{T}_{0}\left|\Lambda^{(s+1) 0}\right\rangle_{(n)_{3}}+\Delta Q\left|\Lambda^{(s+1) 1}\right\rangle_{(n)_{3}} \\
& \mathcal{S}_{(n)_{3}}={ }_{(n)_{3}}\left\langle\tilde{\chi}_{0}^{0}\left|K_{(n)_{3}} \tilde{T}_{0}\right| \chi_{0}^{0}\right\rangle_{(n)_{3}}+\frac{1}{2}{ }_{(n)_{3}}\left\langle\tilde{\chi}_{0}^{1}\left|K_{(n)_{3}}\left\{\tilde{T}_{0}, \eta_{i}^{+} \eta_{i}\right\}\right| \chi_{0}^{1}\right\rangle_{(n)_{3}} \\
& \quad+{ }_{(n)_{3}}\left\langle\tilde{\chi}_{0}^{0}\left|K_{(n)_{3}} \Delta Q\right| \chi_{0}^{1}\right\rangle_{(n)_{3}}+{ }_{(n) 3}\left\langle\tilde{\chi}_{0}^{1}\left|K_{(n)_{3}} \Delta Q\right| \chi_{0}^{0}\right\rangle_{(n)_{3}}
\end{aligned}
$$

where the operator $K_{(s)_{3}}$ is determined by relations (3.16), (4.8), (B.18) for $k=3$. The corresponding Lagrangian formulation is an $\left(n_{1}+n_{2}+n_{3}+2\right)$-th-stage reducible gauge theory for a free arbitrary HS fermionnic field, subject to a Young tableaux $Y\left(n_{1}, n_{2}, n_{3}\right)$ in a Minkowski space $\mathbb{R}^{1, d-1}$. To demonstrate an application of the constructed Lagrangian formulations in the above two examples, we will use our results to find a component Lagrangian formulation for the spin-tensor $\Psi_{\mu \nu, \rho}$ with spin $\mathbf{s}=\left(2+\frac{1}{2}, 1+\frac{1}{2}\right)$.

\subsection{Spin $\left(\frac{5}{2}, \frac{3}{2}\right)$ Mixed-symmetric Massless Spin-tensor}

In this section, the general prescriptions of our Lagrangian formulation will be applied to a rank-3 spin-tensor field, $\Psi_{\mu \nu, \rho} \equiv \Psi_{\mu \nu, \rho A}$, with a suppressed Dirac index $A=1, \ldots, 2^{\left[\frac{d}{2}\right]}$, symmetric in the indices $\mu, \nu$, i.e. $\Psi_{\mu \nu, \rho}=\Psi_{\nu \mu, \rho}$, starting from an analysis of the tower of gauge transformations on a basis of the cohomological resolution complex.

\subsubsection{Reducible Gauge Transformations for Gauge Parameters}

For a spin- $\left(\frac{5}{2}, \frac{3}{2}\right)$ field, we have $\left.\left(h^{1}, h^{2}\right)=\left(\left\{-\frac{d}{2}\right\},\left\{3-\frac{d}{2}\right\}\right\}\right)$. Therefore, due to the analysis of the system of four spin (6.5) and two (6.6) ghost number equations for all the indices of powers in the 
decomposition (5.8) and (2.6) for the fields $\left|\chi_{0}^{l}\right\rangle_{(2,1)}$ and each of the gauge parameters $\left|\Lambda_{0}^{(s) l}\right\rangle_{(2,1)}$, $l=0,1$ and $s=0,1,2,3$, the gauge theory is that of an $(L=3)$-th stage of reducibility.

As the initial step, the first lowest (independent) gauge parameter $\left|\Lambda_{0}^{(3) 0}\right\rangle_{(2,1)}$ is determined only by two-component spinor fields $\psi_{1}^{(3) 0}(x), \psi_{2}^{(3)}{ }_{2}^{0}(x)(\underline{D}$.1 $)$, whereas the parameter $\left|\Lambda_{0}^{(3) 1}\right\rangle_{(2,1)}$ vanishes identically (see Appendix (D) for all explicit expressions of the field $\left|\chi_{0}^{l}\right\rangle_{(2,1)}$ and gauge $\left|\Lambda_{0}^{(s) l}\right\rangle_{(2,1)}$ vectors obtained from the general representation (5.8). For the reducible gauge parameters of the second level $\left|\Lambda_{0}^{(2) l}\right\rangle_{(2,1)}$, given by Eqs. (D.2) - (D.5) $)$, we have the gauge transformations from Eqs. (5.36), (5.37), for $s=2$,

$$
\delta\left|\Lambda_{0}^{(2) 0}\right\rangle_{(2,1)}=\Delta Q\left|\Lambda_{0}^{(3) 0}\right\rangle_{(2,1)}, \quad \delta\left|\Lambda_{0}^{(2) 1}\right\rangle_{(2,1)}=\tilde{T}_{0}\left|\Lambda_{0}^{(3) 0}\right\rangle_{(2,1)} .
$$

Due to Eqs. (6.35), the gauge transformations for the component spin-tensors $\psi^{(2) l}$... have the form (omitting the coordinates $x\left(x \in R^{1, d-1}\right)$ in the arguments)

$$
\begin{aligned}
& \delta \psi_{1}^{(2) 0}=\psi_{1}^{(3) 0}, \\
& \delta \psi_{2}^{(2) 0}=-3 \psi_{1}^{(3) 0}{ }_{1}^{0}, \\
& \delta \psi_{3}^{(2) 0}=\psi_{2}^{(3) 0}, \\
& \delta \psi_{4}^{(2) 0}=-\psi_{2}^{(3) 0} \text {, } \\
& \delta \psi_{5}^{(2) 0}=-3 \psi_{1}^{(3) 0}{ }_{1} \text {, } \\
& \delta \psi_{6}^{(2) 0}=2 \psi_{2}^{(3) 0}, \\
& \delta \psi_{7}^{(2) 0}=0 \text {, } \\
& \delta \psi_{8}^{(2) 0}=-2 \psi_{2}^{(3) 0} \text {, } \\
& \delta \psi_{8 \mid \mu}^{(2) 0}=-2 \gamma_{\mu} \psi_{2}^{(3)}{ }_{2}^{0} \text {, } \\
& \delta \psi_{9}^{(2) 0}=12 \psi_{1}^{(3) 0}, \\
& \delta \psi_{5 \mid \mu}^{(2) 0}=-3 \gamma_{\mu} \psi_{1}^{(3) 0}+\imath \partial_{\mu} \psi_{2}^{(3) 0} \\
& \delta \psi_{10}^{(2) 0}=4 \psi_{2}^{(3) 0} \text {, } \\
& \delta \psi_{2}^{(2) 1}=\imath \gamma^{\mu} \partial_{\mu} \psi_{2}^{(3) 0}+6 \psi_{1}^{(3) 0}, \quad \delta \psi_{1}^{(2) 1}=-\imath \gamma^{\mu} \partial_{\mu} \psi_{1}^{(3) 0} \text {. }
\end{aligned}
$$

In deducing Eqs. (6.36)-(6.40), we use the definition of odd operators $\Delta Q$ (6.8) and $\tilde{T}_{0}(\underline{6.24})$, for $i=1,2$ and $k=2$ in the Young tableaux, as well as the structure of additional parts for the constraints (6.9), (6.10), (6.12), (6.18), (6.19), (6.21), (6.22), (6.24), (6.27), with the only restriction $k=2$.

We then impose the gauge conditions (C.6) for the first lowest dependent gauge parameter $\left|\Lambda_{0}^{(2) 0}\right\rangle_{(2,1)}$, so that the solution of the equation $f_{1}\left|\Lambda_{0}^{(2)}{ }_{0}^{0}\right\rangle_{(2,1)}=0$ is given by a gauged vector $\left|\Lambda_{g 0}^{(2) 0}\right\rangle_{(2,1)}$ with the vanishing spinors $\psi_{p}^{(2)}{ }_{p}, p=5,8$ and the remaining (independent) component spin-tensors in $\left|\Lambda_{g_{0}}^{(2) 0}\right\rangle_{(2,1)},\left|\Lambda_{0}^{(2) 1}\right\rangle_{(2,1)}$. As a result, the theory becomes a second-stage-reducible gauge theory, and the surviving independent gauge parameter does not depend on the auxiliary oscillator $f_{1}^{+}$.

In turn, the general gauge conditions (C.8), (C.11), when applied to the second lowest dependent gauge parameters $\left|\Lambda_{0}^{(1) l}\right\rangle_{(2,1)}$, given by Eqs. (D.6) $-(\underline{D .9})$, are as follows:

$$
f_{1} \mathcal{P}_{11}^{+}\left|\Lambda_{0}^{(1) 0}\right\rangle=0, f_{1}\left|\Lambda_{0}^{(1) 1}\right\rangle=0, b_{11} \mathcal{P}_{11}^{+}\left|\Lambda_{0}^{(1) 0}\right\rangle=0
$$

and imply, first, the vanishing of the component functions $\psi_{p}^{(1)}{ }_{p}, p=5,8$, by means of all the degrees of freedom in the vector $\left|\Lambda_{0}^{(2) l}\right\rangle_{(2,1)}$, so that the gauged parameter $\left|\Lambda^{(1) 1}\right\rangle_{(2,1)}$ has the same structure as $\left|\Lambda_{g_{0}}^{(2) 0}\right\rangle_{(2,1)}$, however, with the opposite Grassmann parity. Second, the requirements (6.41) lead to the vanishing of component functions:

$$
\psi_{n}^{\prime(1) 0}, \psi_{r \mid \mu}^{(1) 0}, \psi_{r}^{(1) 0}, \psi_{t}^{(1) 0}, \quad \text { for } n=1,6 ; r=13,18 ; t=9,10,14,15
$$

in the gauge vector $\left|\Lambda_{0}^{(1)}{ }_{0}^{0}\right\rangle_{(2,1)}$. To find the degrees of freedom for which the component functions in the reduced independent parameter $\left|\Lambda_{g 0}^{(2) 0}\right\rangle_{(2,1)}$ correspond to the vanishing of the above firstlevel components, we should examine the component form of gauge transformations for $\left|\Lambda_{0}^{(1) 0}\right\rangle_{(2,1)}$. They are given by the relations

$$
\delta\left|\Lambda_{0}^{(1) 0}\right\rangle_{(2,1)}=\Delta Q\left|\Lambda_{g 0}^{(2) 0}\right\rangle_{(2,1)}, \quad \delta\left|\Lambda_{g 0}^{(2) 1}\right\rangle_{(2,1)}=\tilde{T}_{0}\left|\Lambda_{g 0}^{(2) 0}\right\rangle_{(2,1)},
$$


which follow from Eqs. (5.36), (5.37) for $s=1$. We do not present here the sequence of component relations following from (6.43), however, the restrictions on $\psi^{(1) 0}$ in (6.42) are due to the degrees of freedom in the spinor $\left|\Lambda_{g 0}^{(2) 0}\right\rangle_{(2,1)}$, related, respectively, to the component spinors $\psi_{1}^{(2) 0}, \psi^{(2) 0}{ }_{3}$; $\psi_{5 \mid \mu}^{(2) 0}, \psi_{8 \mid \mu}^{(2) 0}, \psi_{9}^{(2) 0}, \psi_{10}^{(2) 0} ; \psi_{t}^{(2) 0}$ for $t=2,4,6,7$, which we must set equal to 0 in (D.11) for $\left|\Lambda_{0}^{(1) l}\right\rangle_{(2,1)}$. As a result, all degrees of freedom in the gauge parameters $\left|\Lambda_{g 0}^{(2) 0}\right\rangle_{(2,1)},\left|\Lambda_{0}^{(2) 1}\right\rangle_{(2,1)}$ are used, and the theory becomes a first-stage-reducible gauge theory with the independent parameter $\left|\Lambda_{g 0}^{(1) l}\right\rangle_{(2,1)}, l=0,1$, in which only the component spin-tensors $\psi_{r}^{(1)}{ }_{r}$, for $r=1-4,6,7,9, \psi_{t \mid \mu}^{(1) 1}$, for $t=5,8$ and $\psi_{m \mid \mu \nu}^{(1) 0}, \psi_{n \mid \mu}^{\prime(1) 0}, \psi_{u \mid \mu}^{(1) 0}, \psi_{v}^{(1) 0}$, for $m=13,18 ; n=1,6 ; u=1,6,9,10,14,15,21$; $r=13,18 ; v=1-8,11,12,16,17,19-21$ survive. For the reducible gauge parameters $\left|\Lambda_{0}^{l}\right\rangle_{(2,1)}$, $l=0,1$ determined by Eqs. (D.10) $-(\overline{D .13})$ the general gauge conditions (C.12), having the form $\left(\right.$ for $\left.s_{\max }=3\right)$

$$
\left(f_{1}, b_{11}\right) \mathcal{P}_{11}^{+}\left|\Lambda_{0}^{l}\right\rangle=0, \quad\left(f_{2} \Pi_{p_{1}^{+}}^{0}, b_{12}\right) \mathcal{P}_{11}^{+} \mathcal{P}_{12}^{+}\left|\Lambda_{0}^{0}\right\rangle=0
$$

with $\Pi_{p_{1}^{+}}^{0}$ being the projector onto the $p_{1}^{+}$-independent part of $\left|\Lambda_{0}^{0}\right\rangle$, introduced in Eqs. (C.12)), lead to the vanishing of the component spin-tensors

$$
\begin{aligned}
& \psi_{n}^{\prime 1}, \psi_{r \mid \mu}^{1}, \psi_{r}^{1}, \psi_{t}^{1}, \text { for } n=1,6 ; r=13,18 ; t=9,10,14,15, \\
& \psi_{26 \mid \mu}^{\prime \prime 0}, \psi_{n \mid \mu}^{\prime 0}, \psi_{n \mid \mu}^{0}, \psi_{n}^{\prime 0}, \psi_{26}^{\prime \prime 0}, \psi_{n}^{0}, \text { for } n=1,26 ; \\
& \psi_{r}^{0} \text {, for } r=6,11,17,18,20,23,28,29,34,35 ; \psi_{t \mid \mu}^{0} \text {, for } t=20,23,35 ; \psi_{35 \mid \mu \nu}^{0} .
\end{aligned}
$$

The terms in Eqs. (6.45), due to the explicit form of gauge transformations for the vectors $\left|\Lambda_{0}^{l}\right\rangle$,

$$
\begin{aligned}
& \delta\left|\Lambda_{0}^{0}\right\rangle_{(2,1)}=\Delta Q\left|\Lambda_{g 0}^{(1) 0}\right\rangle_{(2,1)}+\frac{1}{2}\left\{\tilde{T}_{0}, \eta_{i}^{+} \eta_{i}\right\}\left|\Lambda_{g 0}^{(1) 1}\right\rangle_{(2,1)}, \\
& \delta\left|\Lambda_{0}^{1}\right\rangle_{(2,1)}=\tilde{T}_{0} \mid \Lambda_{g 0}^{(1) \underset{g 0}{0}\rangle_{(2,1)}+\Delta Q\left|\Lambda_{g 0}^{(1)}\right\rangle_{(2,1)}}
\end{aligned}
$$

with the independent first-level gauge parameters $\left|\Lambda_{g 0}^{(1) l}\right\rangle_{(2,1)}$, should be examined by means of the final relation in (6.48), by analogy with the components $\psi^{(1)}{ }_{\ldots}$ in Eq. (6.42), so that all degrees of freedom of the reduced vector $\left|\Lambda_{g 0}^{(1) 1}\right\rangle_{(2,1)}$ are used. Next, the vector $\left|\Lambda_{0}^{1}\right\rangle_{(2,1)}$ takes a form similar to the vector $\left|\Lambda_{g 0}^{(1) 0}\right\rangle_{(2,1)}$, however, with the opposite Grassmann parities of its components and partially used gauge transformations (6.48),

$$
\delta\left|\Lambda_{0}^{0}\right\rangle_{(2,1)}=\Delta Q\left|\Lambda_{g 0}^{(1) 0}\right\rangle_{(2,1)}, \quad \delta\left|\Lambda_{g 0}^{1}\right\rangle_{(2,1)}=\tilde{T}_{0}\left|\Lambda_{g 0}^{(1) 0}\right\rangle_{(2,1)} .
$$

Without presenting explicitly the entire system of linear equations for the component spin-tensors (6.46), (6.47) (which must vanish) of $\left|\Lambda_{0}^{0}\right\rangle_{(2,1)}$, following from Eqs. (6.49), we list the results of covariant resolution in terms of the components of $\left|\Lambda_{g 0}^{(1) 0}\right\rangle_{(2,1)}$, being the root of the mentioned system. They are presented in the order corresponding to the appearance of spin-tensors in (6.46), (6.47) as follows:

$$
\begin{aligned}
& \psi_{14 \mid \mu}^{(1) 0}, \psi_{n \mid \mu}^{\prime(1) 0}, \psi_{n \mid \mu}^{(1) 0}, \psi_{11}^{(1) 0}, \psi_{16}^{(1) 0}, \psi_{17}^{(1) 0}, \psi_{n}^{(1) 0} \text {, for } n=1,6 ; \\
& \psi_{r}^{(1) 0} \text {, for } r=2-5,19,20,7,8,12,21 ; \psi_{t \mid \mu}^{(1) 0} \text {, for } t=9,10,21 ; \psi_{13 \mid \mu \nu}^{(1) 0} .
\end{aligned}
$$

As a result, we have only two surviving first-level component spin-tensors $\psi_{15 \mid \mu}^{(1) 0}, \psi_{18 \mid \mu \nu}^{(1) 0}$. Then from the residual gauge transformation for the component spin-tensor $\psi_{26 \mid \mu \nu}^{0}$, remaining in the restricted vector $\left|\psi_{26}^{0}\right\rangle_{(1,1)}$,

$$
\delta \psi_{26 \mid \mu \nu}^{0}=-\psi_{18 \mid \mu \nu}^{(1) 0},
$$


we can remove the second-rank spin-tensor $\psi_{26 \mid \mu \nu}^{0}$ by using the degree of freedom of the parameter $\psi_{18 \mid \mu \nu}^{(1) 0}$. The residual gauge transformations for the remaining zero-level gauge parameter from the restricted vector $\left|\Lambda_{g 0}^{0}\right\rangle_{(2,1)}$ have the form (with a unique first-level parameter spin-tensor $\left.\psi_{15 \mid \mu}^{(1)} \underset{15}{ }\right)$

$$
\begin{array}{lrr}
\delta \psi_{26 \mid \mu, \nu}^{0}=\imath \partial_{\nu} \psi_{15 \mid \mu}^{(1) 0}, & \delta \psi_{23 \mid \mu \nu}^{0}=-\frac{\imath}{2} \partial_{\{\mu} \psi_{15 \mid \nu\}}^{(1) 0}, & \delta \psi_{24}^{0}=\gamma^{\mu} \psi_{15 \mid \mu}^{(1) 0}, \\
\delta \psi_{25}^{0}=-\imath \partial^{\mu} \psi_{15 \mid \mu}^{(1) 0}, & \delta \psi_{15 \mid \mu}^{1}=-\imath \gamma^{\nu} \partial_{\nu} \psi_{15 \mid \mu}^{(1) 0}, & \\
\delta \psi_{n}^{0}=\delta \psi_{31}^{\prime 0}=0, & \delta \psi_{p \mid \mu}^{0}=0, & \delta \psi_{r \mid \mu \nu}^{0}=0, \\
\delta \psi_{35 \mid \mu \nu \rho}^{0}=0, & \delta \psi_{1 \mid \mu, \nu}^{0}=0, & \delta \psi_{1}^{\prime \prime 0}=0, \\
\delta \psi_{1 \mid \mu}^{\prime \prime 0}=0, & \delta \psi_{31 \mid \mu}^{\prime 0}=0, & \delta \psi_{\ldots}^{1}=0,
\end{array}
$$

for $n=2-5,7-10,12-16,19,21,22,24,25,27,30-33 ; p=6,11,17,18,28,29,31,34 ; r=1,20$, where we have presented only those components in the last relation of (6.57) which differ from the ones in Eq. (6.45) and $\psi_{15 \mid \mu}^{1}$.

We are now able to examine the gauge transformation for the fields.

\subsubsection{Gauge Transformations for Fields}

For the gauge-dependent field vectors $\left|\chi_{0}^{l}\right\rangle_{(2,1)}$, conditions (6.5), (6.6) allow one, first, to extract the dependence on the ghost variables, as done in Appendix (D), by Eqs. (D.14), (D.15), whose

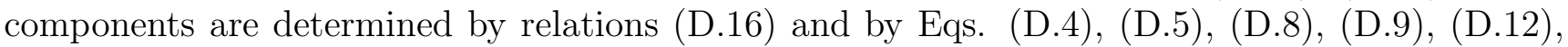
(D.13), however, for the fields vector. The general gauge conditions (C.25) with the operators $\mathcal{C}^{k(k+1)}$, given by (C.24), when applied to the fields in question, read as follows:

$$
\left(\left(f_{1}, b_{11}\right) \mathcal{P}_{11}^{+},\left(f_{2} \Pi_{p_{1}^{+}}^{0}, b_{12}\right) \mathcal{P}_{11}^{+} \mathcal{P}_{12}^{+}\right)\left|\chi_{0}^{l}\right\rangle_{(2,1)}=0, \quad\left(d_{12}\right) \lambda_{12}^{+} \mathcal{P}_{11}^{+} \mathcal{P}_{12}^{+}\left|\chi_{0}^{0}\right\rangle_{(2,1)}=0
$$

Eqs. (6.58) lead, first, to the same elaboration of the field vector $\left|\chi_{0}^{1}\right\rangle_{(2,1)}$ as was done for the gauge parameter $\left|\Lambda_{g 0}^{0}\right\rangle_{(2,1)}$ above, that means, that the only gauge transformations (including trivial ones) with the corresponding component spin-tensors as in Eqs. (6.53)-(6.57) hold true

$$
\begin{array}{lr}
\delta \varphi_{26 \mid \mu, \nu}=\imath \partial_{\nu} \psi_{15 \mid \mu}^{1}+\imath \gamma^{\rho} \partial_{\rho} \psi_{26 \mid \mu, \nu}^{0}, & \delta \varphi_{23 \mid \mu \nu}=-\frac{\imath}{2} \partial_{\{\mu} \psi_{15 \mid \nu\}}^{1}+\imath \gamma^{\rho} \partial_{\rho} \psi_{23 \mid \mu \nu}^{0}, \\
\delta \varphi_{24}=\gamma^{\mu} \psi_{15 \mid \mu}^{1}-\imath \gamma^{\rho} \partial_{\rho} \psi_{24}^{0}-2 \psi_{25}^{0}, & \delta \varphi_{25}=-\imath \partial^{\mu} \psi_{15 \mid \mu}^{1}+\imath \gamma^{\rho} \partial_{\rho} \psi_{25}^{0}, \\
\delta \varphi_{n}=\delta \psi_{31}^{\prime 1}=0, & \delta \varphi_{p \mid \mu}=0, \quad \delta \varphi_{r \mid \mu \nu}=0, \\
\delta \varphi_{35 \mid \mu \nu \rho}=0, & \delta \varphi_{1 \mid \mu, \nu}=0, \quad \delta \varphi_{1}^{\prime \prime}=0, \\
\delta \varphi_{1 \mid \mu}^{\prime \prime}=0, & \delta \varphi_{31 \mid \mu}^{\prime 1}=0, \quad \delta \varphi_{\ldots}=0,
\end{array}
$$

with the same values of indices $n, p, r$ as after Eqs. (6.57). Second, the gauge (6.58) leads to the vanishing of all component spin-tensors in the vector $|\Psi\rangle_{(2,1)}(\mathrm{D.16})$, except for the original spin-tensor $\Psi_{\mu \nu, \rho}$. To this end, the degrees of freedom in the restricted gauge parameter $\left|\Lambda_{g 0}^{0}\right\rangle_{(2,1)}$ corresponding to $\psi_{35 \mid \mu \nu \rho}^{0}, \psi_{1 \mid \mu \nu}^{0}, \psi_{20 \mid \mu \nu}^{0}, \psi_{1 \mid \mu, \nu}^{0}, \psi_{1 \mid \mu}^{\prime \prime 0}, \psi_{31 \mid \mu}^{\prime 0}, \psi_{34 \mid \mu}^{0}, \psi_{31 \mid \mu}^{0}, \psi_{31}^{\prime 0}, \psi_{31}^{0}, \psi_{1}^{\prime \prime 0}$ are used, respectively, for the auxiliary components in (D.16) . Third, this leads to the vanishing of the fields containing the auxiliary oscillators $f_{1}^{+}, b_{11}^{+}, d_{12}^{+}$in the vectors $\left|\psi_{n}\right\rangle_{(0,1)}$ for $n=1,2,14,15 ;\left|\psi_{m}\right\rangle_{(1,0)}$ for $m=3,4,16,17,23,24,26,27,38 ;\left|\psi_{p}\right\rangle_{(2,0)}$ for $p=7,20,36,37 ;\left|\psi_{r}\right\rangle_{(-1,1)}$ for $r=5,18$ and the oscillator $f_{2}^{+}$in the vectors $\left|\psi_{14}\right\rangle_{(0,1)},\left|\psi_{15}\right\rangle_{(0,1)}$. As a result, there remain only the component gauge spin-tensors $\psi_{23 \mid \mu \nu}^{0}, \psi_{26 \mid \mu, \nu}^{0}, \psi_{24}^{0}, \psi_{25}^{0}$ and $\psi_{15 \mid \mu}^{1}$. 
The residual gauge transformations for the restricted field vectors $\left|\chi_{g 0}^{l}\right\rangle_{(2,1)}$

$$
\begin{aligned}
& \delta\left|\chi_{g 0}^{0}\right\rangle_{(2,1)}=\Delta Q\left|\Lambda_{g 0}^{0}\right\rangle_{(2,1)}+\frac{1}{2}\left\{\tilde{T}_{0}, \eta_{i}^{+} \eta_{i}\right\}\left|\Lambda_{g 0}^{1}\right\rangle_{(2,1)} \\
& \delta\left|\chi_{g 0}^{1}\right\rangle_{(2,1)}=\tilde{T}_{0}\left|\Lambda_{g 0}^{0}\right\rangle_{(2,1)}+\Delta Q\left|\Lambda_{g 0}^{1}\right\rangle_{(2,1)}
\end{aligned}
$$

with allowance for the representation

$$
\begin{aligned}
& \frac{1}{2}\left\{\tilde{T}_{0}, \eta_{i}^{+} \eta_{i}\right\}\left|\Lambda_{g 0}^{1}\right\rangle_{(2,1)}=\left[\left(\eta_{1}^{+} \mathcal{P}_{2}^{+}-\eta_{2}^{+} \mathcal{P}_{1}^{+}\right) T_{0}+\left(q_{2}^{+} \mathcal{P}_{1}^{+}-q_{1}^{+} \mathcal{P}_{2}^{+}\right)\right] \tilde{\gamma}\left|\psi_{g \mid 15}^{1}\right\rangle_{(1,0)}, \\
& \tilde{T}_{0}\left|\Lambda_{g 0}^{0}\right\rangle_{(2,1)}=T_{0}\left[\mathcal{P}_{2}^{+}\left(\left|\psi_{g \mid 23}^{0}\right\rangle_{(2,0)}+\mathcal{P}_{1}^{+}\left\{q_{1}^{+} \tilde{\gamma}\left|\psi_{24}^{0}\right\rangle_{(0)}+\eta_{1}^{+}\left|\psi_{25}^{0}\right\rangle_{(0)}\right\}\right)+\mathcal{P}_{1}^{+}\left|\psi_{g \mid 26}^{0}\right\rangle_{(1,1)}\right] \\
& -2 \mathcal{P}_{2}^{+} \mathcal{P}_{1}^{+} q_{1}^{+}\left|\psi_{25}^{0}\right\rangle_{(0)}
\end{aligned}
$$

are written, in addition to (6.59)- 6.63 ) for the component functions with $l=0$, as follows:

$$
\begin{array}{lr}
\delta \Psi_{\mu \nu, \rho}=-\imath \partial_{\rho} \psi_{23 \mid \mu \nu}^{0}-\frac{\imath}{2} \partial_{\{\nu} \psi_{26 \mid \mu\}, \rho}^{0}, & \delta \psi_{14 \mid \mu}=-\imath \partial_{\mu} \psi_{24}^{0}+\gamma^{\nu} \psi_{26 \mid \nu, \mu}^{0}, \\
\delta \psi_{15 \mid \mu}=-\imath \partial_{\mu} \psi_{25}^{0}-\imath \partial^{\nu} \psi_{26 \mid \nu, \mu}^{0}, & \delta \psi_{16 \mid \mu}=\gamma^{\nu} \psi_{26 \mid \mu, \nu}^{0}+\psi_{15 \mid \mu}^{1}, \\
\delta \psi_{17 \mid \mu}=-\imath \partial^{\nu} \psi_{26 \mid \mu, \nu}^{0}-\imath \gamma^{\rho} \partial_{\rho} \psi_{15 \mid \mu}^{1}, & \delta \psi_{19}=-\frac{1}{2} \psi_{\left.26\right|^{\mu}, \mu}^{0}-\frac{1}{2} \psi_{25}^{0}, \\
\delta \psi_{20 \mid \mu \nu}=\frac{1}{2} \psi_{26 \mid\{\nu, \mu\}}^{0}+\psi_{23 \mid \mu \nu}^{0}, & \delta \psi_{26 \mid \mu}=2 \gamma^{\nu} \psi_{23 \mid \mu \nu}^{0}+\imath \partial_{\mu} \psi_{24}^{0}-\psi_{15 \mid \mu}^{1}, \\
\delta \psi_{27 \mid \mu}=-2 \imath \partial^{\nu} \psi_{23 \mid \nu \mu}^{0}+\imath \gamma^{\rho} \partial_{\rho} \psi_{15 \mid \mu}^{1}+\imath \partial_{\mu} \psi_{25}^{0}, & \delta \psi_{28}=-\psi_{23 \mid}^{0}{ }_{\mu}+\psi_{25}^{0}, \\
\delta \psi_{n}=0 & \delta \psi_{p \mid \mu}=\delta \psi_{r \mid \mu \nu}=0,
\end{array}
$$

for $n=1,2,6,8-13,21,22,25,29-35,38 ; p=1-4,23,24,33,36-38 ; r=7,36,37$.

Now, we can reduce the number of the zero-level gauge spin-tensors in Eqs. (6.59)-(6.63), (6.67) -(6.76), expressing the parameters $\psi_{23 \mid \mu \nu}^{0}, \psi_{25}^{0}, \psi_{15 \mid \mu}^{1}$ in terms of the field $\psi_{26 \mid \nu, \mu}^{0}$ only. To do so, we completely remove the field spin-tensors $\psi_{16 \mid \mu}, \psi_{19}, \psi_{20 \mid \mu \nu}$, and therefore obtain

$$
\psi_{15 \mid \mu}^{1}=-\gamma^{\nu} \psi_{26 \mid \mu, \nu}^{0}, \quad \psi_{25}^{0}=-\psi_{26 \mid}^{0}{ }^{\mu}, \mu, \quad \psi_{23 \mid \mu \nu}^{0}=-\frac{1}{2} \psi_{26 \mid\{\nu, \mu\}}^{0} .
$$

As a result, the final non-trivial gauge transformations (6.59)-(6.63), (6.67) -(6.76) take the form

$$
\begin{array}{lr}
\delta \Psi_{\mu \nu, \rho}=\frac{\imath}{2} \partial_{\rho} \psi_{26 \mid\{\nu, \mu\}}^{0}-\frac{\imath}{2} \partial_{\{\nu} \psi_{26 \mid \mu\}, \rho}^{0}, & \delta \psi_{14 \mid \mu}=-\imath \partial_{\mu} \psi_{24}^{0}+\gamma^{\nu} \psi_{26 \mid \nu, \mu}^{0}, \\
\delta \psi_{17 \mid \mu}=\imath \gamma^{\rho \nu} \partial_{\rho} \psi_{26 \mid \mu, \nu}^{0}, & \delta \psi_{26 \mid \mu}=\imath \partial_{\mu} \psi_{24}^{0}-\gamma^{\nu} \psi_{26 \mid \nu, \mu}^{0}, \\
\delta \psi_{27 \mid \mu}=\imath \partial_{[\nu} \psi_{\left.\left.26\right|^{0}, \mu\right]}^{0}-\imath \gamma^{\rho \nu} \partial_{\rho} \psi_{26 \mid \mu, \nu}^{0}, & \delta \psi_{15 \mid \mu}=\imath \partial_{\mu} \psi_{26 \mid}^{0}{ }^{\nu}{ }_{, \nu}-\imath \partial^{\nu} \psi_{26 \mid \nu, \mu}^{0}, \\
\delta \varphi_{26 \mid \mu, \nu}=\imath \gamma^{\rho} \partial_{[\rho} \psi_{26 \mid \hat{\mu}, \nu]}^{0}, & \delta \varphi_{23 \mid \mu \nu}=\frac{\imath}{2} \gamma^{\rho} \partial_{\{\mu} \psi_{26 \mid \nu\}, \rho}^{0}-\frac{\imath}{2} \gamma^{\rho} \partial_{\rho} \psi_{26 \mid\{\mu, \nu\}}^{0}, \\
\delta \varphi_{24}=\psi_{26 \mid}^{0}{ }^{\mu}{ }_{, \mu}-\gamma^{\mu \nu} \psi_{26 \mid \mu, \nu}^{0}-\imath \gamma^{\rho} \partial_{\rho} \psi_{24}^{0}, & \delta \varphi_{25}=\imath \partial^{\mu} \gamma^{\nu} \psi_{26 \mid \mu, \nu}^{0}-\imath \gamma^{\rho} \partial_{\rho} \psi_{26 \mid}^{0}{ }^{\mu}, \mu
\end{array}
$$

where we have introduced the matrix $\gamma^{\rho \nu}=\frac{1}{2}\left(\gamma^{\rho} \gamma^{\nu}-\gamma^{\nu} \gamma^{\rho}\right)$, and the field $\psi_{28}$ becomes gaugeindependent. Finally, due to the presence of the gauge parameter $\psi_{24}^{0}$ only with the derivative $\partial_{\mu}$, we can remove the spin-tensor $\psi_{14 \mid \mu}$ by means of the degree of freedom corresponding to $\psi_{24}^{0}$. After that, we have an algebraic gamma-trace constraint for the gauge parameter $\psi_{26 \mid \nu, \mu}^{0}$,

$$
\gamma^{\nu} \psi_{26 \mid \nu, \mu}^{0}=\imath \partial_{\mu} \psi_{24}^{0},
$$

whose substitution into the gauge transformations for the spin-tensors $\psi_{26 \mid \mu}, \varphi_{24}$ makes them gauge-independent. Notice that the only remaining gauge 2 -nd rank spin-tensor is $\psi_{26 \mid \mu, \nu}^{0}$, however, it is not arbitrary due to the gauge relation (6.53) and the algebraic constraint (6.79).

Let us now turn to the removal of the remaining auxiliary fields from the field vectors $\left|\chi_{g 0}^{l}\right\rangle_{(2,1)}$, by solving some of the equations of motion. 


\subsubsection{Gauge-invariant Unconstrained Lagrangian}

We should find the action of the operator $\Delta Q$ on the gauged field vectors $\left|\chi_{g 0}^{l}\right\rangle_{(2,1)}, l=0,1$, and of the operators $\tilde{T}_{0},\left\{\tilde{T}_{0}, \eta_{i}^{+} \eta_{i}\right\}$, respectively, on $\left|\chi_{g 0}^{0}\right\rangle_{(2,1)}$ and $\left|\chi_{g 0}^{1}\right\rangle_{(2,1)}$, in order to solve the algebraic part of the equations of motion in the last general relation (6.30) having, in this case, the form

$$
\Delta Q\left|\chi_{g 0}^{0}\right\rangle_{(2,1)}+\frac{1}{2}\left\{\tilde{T}_{0}, \eta_{i}^{+} \eta_{i}\right\}\left|\chi_{g 0}^{1}\right\rangle_{(2,1)}=0, \quad \tilde{T}_{0}\left|\chi_{g 0}^{0}\right\rangle_{(2,1)}+\Delta Q\left|\chi_{g 0}^{1}\right\rangle_{(2,1)}=0 .
$$

Our starting point is to solve the final part of Eqs. (6.80), similar to the case of the gauged vector $\left|\Lambda_{g 0}^{0}\right\rangle_{(2,1)}$ in Section 6.3.2. In ding so, we have, due to the obvious consequence that all the fields $\left|\varphi_{n}\right\rangle_{\ldots}$ for $n=16,27,31-34$ with the multipliers $\mathcal{P}_{11}^{+}, \mathcal{P}_{12}^{+}$should vanish, except for the already gauged spinors $\varphi_{34}$, as well as with the linear (in $p_{i}^{+}$) spinors $\left|\varphi_{m}\right\rangle_{\ldots}, m=1,20$ (used earlier for the gauge-fixing of the vectors $\left.\left|\chi_{0}^{l}\right\rangle_{(2,1)}\right)$. Recalling that the only spinor $\psi_{33}$ in the gauged field vector $\left|\chi_{g 0}^{0}\right\rangle_{(2,1)}$ depends on the $f_{1}^{+}$oscillator, we can choose for a solution of the last relation in (6.80) all the vectors $\left|\varphi_{m}\right\rangle \ldots$ for $m=2, \ldots, 19$, equal to 0 . For the same reason (considering $f_{2}^{+}$, the oscillator arguments for the corresponding terms in $\left.\left|\chi_{g 0}^{0}\right\rangle_{(2,1)}\right)$, the vectors $\left|\varphi_{2} 1\right\rangle_{0},\left|\varphi_{2} 2\right\rangle_{0}$ should vanish, as well. Then, the remaining terms, i.e., $\left|\varphi_{p}\right\rangle_{\ldots}, p=28-30,35$, proportional to the momenta $\lambda_{12}^{+}$, should also vanish, because of the corresponding terms in $\tilde{T}_{0}\left|\chi_{g 0}^{0}\right\rangle_{(2,1)}$ (see Eqs. (6.80) ) do not depend on the $d_{12}^{+}$oscillator. Therefore, only the four vectors $\left|\varphi_{r}\right\rangle \ldots, r=23, \ldots, 26$, proportional to the momenta $\mathcal{P}_{i}^{+}$, survive at this stage of resolution of the equations of motion (6.80).

Turning to the first relation in (6.80), we can see that, due to the absence of the auxiliary oscillators $f_{i}^{+}, b_{i j}^{+}, d_{12}^{+}$in the final field vector $\left|\chi_{g 0}^{1}\right\rangle_{(2,1)}$, all the vectors $\left|\psi_{n}\right\rangle_{\ldots}$, with any of the multipliers $\mathcal{P}_{i j}^{+}, \lambda_{12}^{+}, p_{i}^{+}$should vanish. Summarizing, the result of Eqs. (66.80) resolution permits one to present the vectors $\left|\chi_{g 0}^{l}\right\rangle_{(2,1)}, l=0,1$, in the form

$$
\begin{aligned}
\left|\chi_{g 0}^{1}\right\rangle_{(2,1)}= & \mathcal{P}_{2}^{+}\left(\tilde{\gamma}\left|\varphi_{g \mid 23}\right\rangle_{(2,0)}+\mathcal{P}_{1}^{+}\left\{q_{1}^{+}\left|\varphi_{24}\right\rangle_{(0)}+\eta_{1}^{+} \tilde{\gamma}\left|\varphi_{25}\right\rangle_{(0)}\right\}\right)+\mathcal{P}_{1}^{+} \tilde{\gamma}\left|\varphi_{g \mid 26}\right\rangle_{(1,1)}, \\
\left|\chi_{g 0}^{0}\right\rangle_{(2,1)}= & \left|\Psi_{g}\right\rangle_{(2,1)}+\mathcal{P}_{1}^{+}\left(\eta_{1}^{+}\left|\psi_{g \mid 15}\right\rangle_{(0,1)}+\eta_{2}^{+}\left|\psi_{g \mid 17}\right\rangle_{(1,0)}\right) \\
& +\mathcal{P}_{2}^{+}\left(q_{1}^{+} \tilde{\gamma}\left|\psi_{g \mid 26}\right\rangle_{(1,0)}+\eta_{1}^{+}\left|\psi_{g \mid 27}\right\rangle_{(1,0)}+\eta_{11}^{+}\left|\psi_{28}\right\rangle_{(0)}\right) .
\end{aligned}
$$

The corresponding unconstrained gauge-invariant Lagrangian for the spin-tensor $\Psi_{\mu \nu, \rho}$ with auxiliary 2 second rank spin-tensor fields, $\varphi_{23 \mid \mu \nu}, \varphi_{26 \mid \mu, \nu}, 4$ first rank spin-tensors $\psi_{15 \mid \mu}, \psi_{17 \mid \mu}, \psi_{26 \mid \mu}, \psi_{27 \mid \mu}$ and 3 spinors $\varphi_{24}, \varphi_{25}, \psi_{28}$ from Eqs. (6.81), (6.82) in terms of the odd scalar product in $\mathcal{H} \otimes \mathcal{H}_{g h}$ read as follows:

$$
\begin{aligned}
\mathcal{S}_{(2,1)}= & { }_{(2,1)}\left\langle\tilde{\chi}_{g 0}^{0}\left|\tilde{T}_{0}\right| \chi_{g 0}^{0}\right\rangle_{(2,1)}+\frac{1}{2}{ }_{(2,1)}\left\langle\tilde{\chi}_{g 0}^{1}\left|\left\{\tilde{T}_{0}, \eta_{i}^{+} \eta_{i}\right\}\right| \chi_{g 0}^{1}\right\rangle_{(2,1)} \\
& +{ }_{(2,1)}\left\langle\tilde{\chi}_{g 0}^{0}|\Delta Q| \chi_{g 0}^{1}\right\rangle_{(2,1)}+{ }_{(2,1)}\left\langle\tilde{\chi}_{g 0}^{1}|\Delta Q| \chi_{g 0}^{0}\right\rangle_{(n)_{3}} .
\end{aligned}
$$

Now, we continue to eliminate the auxiliary fields from the configuration space by solving the basic part of the remaining equations of motion, following from the action (6.83), with allowance for the representation

$$
\begin{aligned}
& \frac{1}{2}\left\{\tilde{T}_{0}, \eta_{i}^{+} \eta_{i}\right\}\left|\chi_{g 0}^{1}\right\rangle_{(2,1)}=T_{0} \eta_{1}^{+}\left[\tilde{\gamma}\left|\varphi_{g \mid 26}\right\rangle_{(1,1)}-\mathcal{P}_{2}^{+} q_{1}^{+}\left|\varphi_{24}\right\rangle_{(0)}\right]+T_{0} \eta_{2}^{+}\left[\tilde{\gamma}\left|\varphi_{g \mid 23}\right\rangle_{(2,0)}\right. \\
& \left.\quad+\mathcal{P}_{1}^{+}\left\{q_{1}^{+}\left|\varphi_{24}\right\rangle_{(0)}+\eta_{1}^{+} \tilde{\gamma}\left|\varphi_{25}\right\rangle_{(0)}\right\}\right]+q_{1}^{+}\left(\eta_{1}^{+} \mathcal{P}_{2}^{+}-2 \eta_{2}^{+} \mathcal{P}_{1}^{+}\right) \tilde{\gamma}\left|\varphi_{25}\right\rangle_{(0)}-q_{1}^{+}\left[\tilde{\gamma}\left|\varphi_{g \mid 26}\right\rangle_{(1,1)}\right. \\
& \left.\quad-\mathcal{P}_{2}^{+} q_{1}^{+}\left|\varphi_{24}\right\rangle_{(0)}\right]-q_{2}^{+}\left[\tilde{\gamma}\left|\varphi_{g \mid 23}\right\rangle_{(2,0)}+\mathcal{P}_{1}^{+}\left\{q_{1}^{+}\left|\varphi_{24}\right\rangle_{(0)}+\eta_{1}^{+} \tilde{\gamma}\left|\varphi_{25}\right\rangle_{(0)}\right\}\right] \\
& \tilde{T}_{0}\left|\chi_{g 0}^{0}\right\rangle_{(2,1)}=T_{0}\left|\chi_{g 0}^{0}\right\rangle_{(2,1)}+2 q_{1}^{+}\left[\mathcal{P}_{1}^{+}\left|\psi_{g \mid 15}\right\rangle_{(0,1)}+\mathcal{P}_{2}^{+}\left|\psi_{g \mid 27}\right\rangle_{(1,0)}\right]+2 q_{2}^{+} \mathcal{P}_{1}^{+}\left|\psi_{g \mid 17}\right\rangle_{(1,0)} .
\end{aligned}
$$


The equations of motion (except for ghost-independent terms) for the second relation in Eqs. (6.80) have the form

$$
\begin{aligned}
& 2 \psi_{15 \mid \mu}-\imath \partial_{\mu} \varphi_{24}+\gamma^{\nu} \varphi_{26 \mid \nu, \mu}=0, \\
& -\varphi_{23 \mid}{ }^{\mu}{ }_{\mu}-\varphi_{25}-\imath \gamma^{\mu} \partial_{\mu} \psi_{28}=0, \\
& \imath \gamma^{\rho} \partial_{\rho} \psi_{15 \mid \mu}+\imath \partial_{\mu} \varphi_{25}+\imath \partial^{\nu} \varphi_{26 \mid \nu, \mu}=0, \\
& \gamma^{\nu} \varphi_{26 \mid \mu, \nu}+2 \psi_{17 \mid \mu}=0, \\
& -\imath \partial^{\nu} \varphi_{26 \mid \mu, \nu}-\imath \gamma^{\rho} \partial_{\rho} \psi_{17 \mid \mu}=0, \\
& -\frac{1}{2} \varphi_{26}{ }^{\mu}{ }_{, \mu}-\frac{1}{2} \varphi_{25}=0, \\
& \frac{1}{2} \varphi_{26 \mid\{\nu, \mu\}}+\varphi_{23 \mid \mu \nu}=0, \\
& 2 \gamma^{\nu} \varphi_{23 \mid \mu \nu}+\imath \partial_{\mu} \varphi_{24}+2 \psi_{27 \mid \mu}+\imath \gamma^{\rho} \partial_{\rho} \psi_{26 \mid \mu}=0 \text {, } \\
& -2 \imath \partial^{\nu} \varphi_{23 \mid \nu \mu}+\imath \partial_{\mu} \varphi_{25}-\imath \gamma^{\rho} \partial_{\rho} \psi_{27 \mid \mu}=0 .
\end{aligned}
$$

For the first generating equation in (6.80), we have

$$
\begin{array}{lr}
-\imath \partial^{\rho} \Psi_{\mu \nu, \rho}-\frac{\imath}{2} \partial_{\{\mu} \psi_{17 \mid \nu\}}+\imath \gamma^{\rho} \partial_{\rho} \varphi_{26 \mid \mu, \nu}=0, & \Psi_{\{\mu \nu, \rho\}}=0, \\
-2 \gamma^{\rho} \Psi_{\mu \rho, \nu}-\imath \partial_{\nu} \psi_{26 \mid \mu}-\varphi_{26 \mid \mu, \nu}=0, & -\gamma^{\rho} \Psi_{\mu \nu, \rho}-\varphi_{23 \mid \mu \nu}=0, \\
2 \imath \partial^{\rho} \Psi_{\mu \rho, \nu}+\imath \partial_{\mu} \psi_{15 \mid \nu}+\imath \partial_{\nu} \psi_{27 \mid \mu}-\imath \gamma^{\rho} \partial_{\rho} \varphi_{23 \mid \mu \nu}=0, & \Psi_{\rho, \mu}^{\rho}-\imath \partial_{\mu} \psi_{28}-\psi_{15 \mid \mu}=0, \\
\frac{1}{2} \Psi_{\mu, \rho}^{\rho}-\frac{1}{2} \psi_{27 \mid \mu}-\frac{1}{2} \psi_{17 \mid \mu}=0, & \psi_{26 \mid \mu}=0, \\
\imath \gamma^{\rho} \partial_{\rho} \varphi_{25}+\imath \partial^{\rho} \psi_{15 \mid \rho}-\imath \partial^{\rho} \psi_{17 \mid \rho}=0, & -\psi_{15 \mid \mu}-\psi_{17 \mid \mu}-\psi_{27 \mid \mu}=0, \\
4 \psi_{28}+\varphi_{24}+\gamma^{\rho} \psi_{26 \mid \rho}=0, & -\varphi_{24}=0, \\
-\varphi_{25}-\gamma^{\nu} \psi_{15 \mid \nu}=0, & 2 \varphi_{25}+\imath \gamma^{\rho} \partial_{\rho} \varphi_{24}-\gamma^{\nu} \psi_{17 \mid \nu}=0, \\
-\varphi_{25}-\imath \gamma^{\rho} \partial_{\rho} \varphi_{24}-\gamma^{\nu} \psi_{27 \mid \nu}-\imath \partial^{\rho} \psi_{26 \mid \rho}=0 . &
\end{array}
$$

From the second equation in (6.91), which implies the Young symmetry condition for the initial field $\Psi_{\mu \nu, \rho}$, we can compose the spin-tensor

$$
\widetilde{\Psi}_{\mu \nu, \rho}=\Psi_{\mu \nu, \rho}-\frac{1}{2} \Psi_{\rho \mu, \nu}-\frac{1}{2} \Psi_{\nu \rho, \mu}
$$

identically satisfying Eq. (6.91). Therefore, the solution of the system (6.86)-(6.98) with respect to the spin-tensor $\widetilde{\Psi}_{\mu \nu, \rho}$ has the form

$$
\begin{aligned}
& \varphi_{23 \mid \mu \nu}=-\gamma^{\rho} \Psi_{\mu \nu, \rho}, \varphi_{26 \mid \mu, \nu}=-2 \gamma^{\rho} \Psi_{\mu \rho, \nu}, \varphi_{25}=2 \gamma^{\rho} \Psi_{\rho, \mu}^{\mu}, \psi_{17 \mid \mu}=\gamma^{\nu} \gamma^{\rho} \Psi_{\mu \rho, \nu} \\
& \psi_{15 \mid \mu}=\Psi_{\nu, \mu}^{\nu}, \quad \psi_{27 \mid \mu}=\gamma^{\nu} \gamma^{\rho} \Psi_{\mu \nu, \rho}, \varphi_{24}=\psi_{26 \mid \mu}=\psi_{28}=0
\end{aligned}
$$

From the Young symmetry relation, there follows the validity of the algebraic consequences $\Psi_{\nu, \mu}^{\nu}=-2 \Psi_{\mu, \nu}^{\nu}$ for the spin-tensor $\Psi_{\mu \nu, \rho}$ components.

The final bra-vectors ${ }_{(2,1)}\left\langle\tilde{\chi}_{g 0}^{l}\right|$ read, due to the general formula (6.83) for the action, with allowance for the relations (6.100) and the Hermitian conjugation rule, using the matrix $F^{+}=$ $\tilde{\gamma}^{0}(F)^{+} \tilde{\gamma}^{0}$ and the spin-tensor $\psi^{+}=(\psi)^{+} \tilde{\gamma}^{0}$, being compatible with the conventional one [55], [56]

$$
\begin{aligned}
{ }_{(2,1)}\left\langle\tilde{\chi}_{g 0}^{0}\right| & ={ }_{(2,1)}\left\langle\tilde{\Psi}_{g}\right|+{ }_{(0,1)}\left\langle\tilde{\psi}_{g \mid 15}\right| \eta_{1} \mathcal{P}_{1}+{ }_{(1,0)}\left\langle\tilde{\psi}_{g \mid 17}\right| \eta_{2} \mathcal{P}_{1}+{ }_{(1,0)}\left\langle\tilde{\psi}_{g \mid 27}\right| \eta_{1} \mathcal{P}_{2} \\
{ }_{(2,1)}\left\langle\tilde{\chi}_{g 0}^{1}\right| & ={ }_{(2,0)}\left\langle\tilde{\varphi}_{g \mid 23}\right| \tilde{\gamma} \mathcal{P}_{2}+{ }_{(0,0)}\left\langle\tilde{\varphi}_{25}\right| \tilde{\gamma}_{1} \eta_{1} \mathcal{P}_{2}+{ }_{(1,1)}\left\langle\tilde{\varphi}_{g \mid 26}\right| \tilde{\gamma} \mathcal{P}_{1},
\end{aligned}
$$

with the component bra-vectors

$$
\begin{array}{lrl}
{ }_{(2,1)}\left\langle\tilde{\Psi}_{g}\right|=\langle 0| a_{1}^{\mu} a_{1}^{\nu} a_{2}^{\rho} \Psi_{\mu \nu, \rho}^{+}(x) \tilde{\gamma}_{0}, & { }_{(0,1)}\left\langle\tilde{\psi}_{g \mid 15}\right|=\langle 0| \psi_{15 \mid \mu}^{+}(x) a_{2}^{\mu} \tilde{\gamma}_{0}, \\
{ }_{(2,0)}\left\langle\tilde{\varphi}_{g \mid 23}\right|=\langle 0| \varphi_{23 \mid \mu \nu}^{+}(x) a_{1}^{\nu} a_{1}^{\mu} \tilde{\gamma}_{0}, & { }_{(1,1)}\left\langle\tilde{\varphi}_{g \mid 26}\right|=\langle 0| \varphi_{26 \mid \mu, \nu}^{+}(x) a_{2}^{\nu} a_{1}^{\mu} \tilde{\gamma}_{0}, \\
{ }_{(1,0)}\left\langle\tilde{\psi}_{g \mid 27}\right|=\langle 0| \psi_{27 \mid \mu}^{+}(x) a_{1}^{\mu} \tilde{\gamma}_{0}, & (0,0)\left\langle\tilde{\psi}_{28}\right|=\langle 0| \psi_{28}^{+}(x) \tilde{\gamma}_{0} .
\end{array}
$$


Explicitly, the action (6.83) in terms of the spin-tensor $\Psi_{\mu \nu, \rho}$ and auxiliary spin-tensors has the form

$$
\begin{aligned}
& \mathcal{S}_{(2,1)}=\int d^{d} x\left\{\bar{\Psi}_{\mu \nu, \rho} \imath \gamma^{\tau} \partial_{\tau} \Psi^{\mu \nu, \rho}-\bar{\psi}_{15 \mid \mu} \imath \gamma^{\tau} \partial_{\tau} \psi_{\left.15\right|^{\mu}}-\bar{\psi}_{17 \mid \mu} \imath \gamma^{\tau} \partial_{\tau} \psi_{17 \mid}{ }^{\mu}-\bar{\psi}_{27 \mid \mu} \imath \gamma^{\tau} \partial_{\tau} \psi_{\left.27\right|^{\mu}}\right. \\
& +2 \bar{\varphi}_{23 \mid \mu \nu} \imath \gamma^{\tau} \partial_{\tau} \varphi_{23 \mid}{ }^{\mu \nu}+\bar{\varphi}_{26 \mid \mu, \nu} \imath \gamma^{\tau} \partial_{\tau} \varphi_{26 \mid}^{\mu, \nu}-\bar{\varphi}_{25} \imath \gamma^{\tau} \partial_{\tau} \varphi_{25}+2 \imath \bar{\Psi}_{\mu \nu, \rho} \partial^{\rho} \varphi_{23 \mid}{ }^{\mu \nu} \\
& \left.+\imath \bar{\Psi}_{\mu \nu, \rho} \partial^{\{\nu} \varphi_{26 \mid} \mu\right\}, \rho-\imath \bar{\psi}_{15 \mid \mu}\left(\partial_{\nu} \varphi_{26 \mid}^{\nu, \mu}+\partial^{\mu} \varphi_{25}\right)-\left.\imath \bar{\psi}_{17 \mid\{\mu} \partial_{\nu\}} \varphi_{23 \mid}\right|^{\nu \mu} \\
& +\imath \bar{\psi}_{17 \mid \mu} \partial^{\mu} \varphi_{25}-\imath \bar{\psi}_{27 \mid \mu} \partial_{\nu} \varphi_{26 \mid}^{\mu, \nu}+2 \imath \bar{\varphi}_{23 \mid}^{\mu \nu} \partial^{\rho} \Psi_{\mu \nu, \rho}-\imath \bar{\varphi}_{23 \mid}{ }^{\nu \mu} \partial_{\{\nu} \psi_{17 \mid \mu\}} \\
& \left.-\imath \bar{\varphi}_{25} \partial^{\mu}\left(\psi_{15 \mid \mu}-\psi_{17 \mid \mu}\right)+\imath \bar{\varphi}_{26 \mid\{\mu, \rho} \partial_{\nu\}} \Psi^{\mu \nu, \rho}-\imath \bar{\varphi}_{26 \mid}^{\mu, \nu}\left(\partial_{\mu} \psi_{15 \mid \nu}+\partial_{\nu} \psi_{27 \mid \mu}\right)\right\} .
\end{aligned}
$$

The above action determines a gauge-invariant Lagrangian description for a free massless fermionic particle of generalized spin $\left(\frac{5}{2}, \frac{3}{2}\right)$, described by a field $\Psi_{\mu \nu, \rho}$ and a set of auxiliary spin-tensors $\varphi_{23 \mid \mu \nu}, \varphi_{25}, \varphi_{26 \mid \mu, \nu}, \psi_{15 \mid \mu}, \psi_{17 \mid \mu}, \psi_{27 \mid \mu}$. The reducible gauge transformations are given by Eqs. (6.74)(6.77) with the reducibility and constraint conditions in (6.53) and (6.79), respectively.

The action (6.107) is easily expressed through the spin-tensor $\Psi_{\mu \nu, \rho}$ components only, in view of Eqs. (6.100), (6.101):

$$
\begin{aligned}
\mathcal{S}_{(2,1)}= & \int d^{d} x \bar{\Psi}_{\mu \nu, \rho}\left\{\imath \gamma^{\tau} \partial_{\tau} \Psi^{\mu \nu, \rho}-\eta^{\mu \nu} \imath \gamma^{\tau} \partial_{\tau} \eta^{\sigma \lambda} \Psi_{\sigma \lambda,}{ }^{\rho}-\gamma^{\nu} \gamma^{\rho} \imath \gamma^{\tau} \partial_{\tau} \gamma^{\lambda} \gamma^{\sigma} \Psi_{\sigma, \lambda}^{\mu}\right. \\
& -\gamma^{\rho} \gamma^{\nu} \imath \gamma^{\tau} \partial_{\tau} \gamma^{\sigma} \gamma^{\lambda} \Psi_{\sigma, \lambda}^{\mu}+2 \gamma^{\rho} \imath \gamma^{\tau} \partial_{\tau} \gamma^{\sigma} \Psi^{\mu \nu}{ }_{\sigma}+4 \gamma^{\nu} \imath \gamma^{\tau} \partial_{\tau} \gamma_{\sigma} \Psi^{\mu \sigma, \rho}-4 \imath \partial^{\rho} \gamma^{\sigma} \Psi^{\mu \nu}{ }_{\sigma}{ }_{\sigma} \\
& -\eta^{\mu \nu} \gamma^{\rho} \imath \gamma^{\tau} \partial_{\tau} \eta^{\sigma \lambda} \gamma^{\alpha} \Psi_{\sigma \lambda, \alpha}-4 \imath \partial^{\{\nu} \gamma^{\sigma} \Psi^{\mu\}}{ }_{\sigma,}{ }^{\rho}+4 \imath \eta^{\mu \nu}\left(\partial_{\sigma} \gamma^{\lambda} \Psi_{\lambda}{ }^{\sigma, \rho}-\partial^{\rho} \gamma^{\sigma} \eta^{\tau \alpha} \Psi_{\tau \sigma, \alpha}\right) \\
& \left.+4 \imath \gamma^{\nu} \gamma^{\rho} \partial_{\sigma} \gamma^{\tau} \Psi_{, \tau}^{\mu \sigma}+4 \imath \gamma^{\nu} \gamma^{\rho} \partial^{\mu} \eta^{\sigma \lambda} \gamma^{\alpha} \Psi_{\sigma \alpha, \lambda}+4 \imath \gamma^{\rho} \gamma^{\nu} \partial_{\sigma} \gamma^{\alpha} \Psi_{\alpha}{ }^{\mu, \sigma}\right\},
\end{aligned}
$$

and is invariant with respect to the reducible gauge transformations

$$
\delta \Psi_{\mu \nu, \rho}=\frac{\imath}{2} \partial_{\rho} \xi_{\{\nu, \mu\}}-\frac{\imath}{2} \partial_{\{\nu} \xi_{\mu\}, \rho}, \quad \delta \xi_{\mu, \nu}=\imath \partial_{\nu} \xi_{\mu}^{(1)}, \quad\left(\xi_{\mu, \nu}, \xi_{\mu}^{(1)}\right) \equiv\left(\psi_{26 \mid \mu, \nu}^{0}, \psi_{15 \mid \mu}^{(1) 0}\right),
$$

subject to the relation $\gamma^{\mu} \xi_{\mu, \nu}=\imath \partial_{\nu} \xi$, with an arbitrary spinor $\xi$.

We have thus obtained the gauge-invariant Lagrangian (6.108) only in terms of the initial free massless mixed-symmetric spin-tensor field $\Psi_{\mu \nu, \rho}$. The resulting theory is a first-stage reducible gauge theory. The formulae (6.108), (6.109) present our basic result of Section 6.3.

In view of the above result (see also the result for the antisymmetric spin-tensor $\Psi_{[\mu, \nu]}$ in [59]), it should be noted that any gauge-invariant unconstrained Lorentz-covariant Lagrangian formulation for the fermionic mixed-symmetric spin-tensor $\Psi_{\left(\mu^{1}\right)_{n_{1}},\left(\mu^{2}\right)_{n_{2}}, \ldots,\left(\mu^{k}\right)_{n_{k}}}$ in Minkowski space realized only in terms of the initial spin-tensor $\Psi_{\left(\mu^{1}\right)_{n_{1}},\left(\mu^{2}\right)_{n_{2}}, \ldots,\left(\mu^{k}\right)_{n_{k}}}$ (i.e., without any auxiliary fields) must possess a reducible gauge symmetry transformation in such a way that the stage of reducibility should be equal to $(k-1)$ for a field corresponding to a Young tableaux $Y\left(s_{1}, \ldots, s_{k}\right)$. This obvious property is in contradiction with the Lagrangian formulation suggested for fermionic fields in the "metric-like" formalism [65] in a flat space-time, where the resulting Lagrangian formulation for any spin-tensor is an irreducible gauge theory ${ }^{14}$. A similar conclusion can be immediately enlarged for bosonic mixed-symmetric fields in Minkowski space subject to $Y\left(s_{1}, \ldots, s_{k}\right)$, whose unconstrained Lagrangian formulation was suggested in our previous paper [31. Indeed, the Lagrangian formulation for a fourth-rank tensor $\Phi_{\mu \nu, \rho, \sigma}$ suggested therein is a second-stage reducible gauge theory, whereas a general unconstrained Lagrangian for any mixed-symmetric tensor in [66] has independent gauge parameters only; meanwhile, no Lagrangian for a specific mixed-symmetric tensor was presented.

\footnotetext{
${ }^{14}$ The author is grateful to W.Siegel for an explanation of the peculiarities of the formalism of [65, which, unfortunately, was not provided by any explicit example of Lagrangians for mixed-symmetric spin-tensors, and, in addition, is based on the hypothesis that all the algebraic gamma-traceless and mixed-symmetry constraints for any initial spin-tensor be resolved before the Lagrangian is derived.
} 


\subsection{Spin $\left(\frac{5}{2}, \frac{3}{2}\right)$ Mixed-symmetric Massive Spin-tensor}

To obtain a Lagrangian description of a massive rank-3 mixed-symmetric spin-tensor $\Psi_{\mu \nu, \rho}$, having

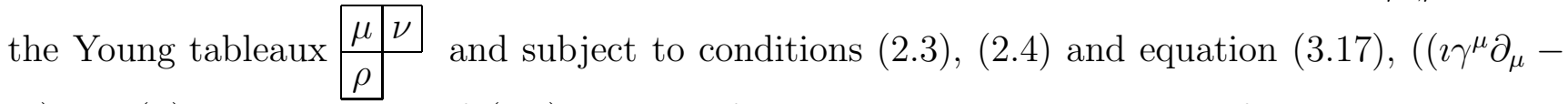
m) $\Psi_{\mu \nu, \rho}(x)=0$, instead of (2.2), we can follow, in part, the example of a massive secondrank antisymmetric spin-tensor from Ref. [59], and apply the prescription (3.18), (3.19), starting directly from the massless Lagrangian formulation with the action (6.108) in a $(d+1)$-dimensional Minkowski space.

First, we have the following representation for the field and gauge parameters:

$$
\begin{aligned}
\Psi^{M N, P} & =\left(\Psi^{\mu \nu, \rho}, \Psi^{\mu \nu, d}, \Psi^{\mu d, \rho}, \Psi^{\mu d, d}, \Psi^{d d, \rho}, \Psi^{d d, d}\right) \\
\xi_{M, N} & =\left(\xi_{\mu, \nu}, \xi_{\mu, d}, \xi_{d, \nu}, \xi_{d, d}\right), \quad \xi_{M}^{(1)}=\left(\xi_{\mu}^{(1)}, \xi_{d}^{(1)}\right) .
\end{aligned}
$$

The Young symmetry condition for the spin-tensor $\Psi^{M N, P}$, i.e., $\Psi^{\{M N, P\}}=0$, implies that after a projection onto $\mathbb{R}^{1, d-1}$ the $d$-dimensional spinor $\Psi^{d d, d}=\frac{1}{3} \Psi^{\{d d, d\}}$ vanishes, and there hold the following properties of the remaining projected spin-tensors (6.110):

$$
\Psi^{d d, \mu}=-2 \Psi^{\mu d, d}, \quad \Psi^{\mu \nu, d}=-\Psi^{d\{\mu, \nu\}}, \quad \Psi^{\{\mu \nu, \rho\}}=0 .
$$

Therefore, the total configuration space contains one third-rank massive mixed-symmetric spintensor $\Psi^{\mu \nu, \rho}$, two second-rank symmetric $\varphi_{1}^{\mu \nu}, \varphi_{1}^{\mu \nu} \equiv \Psi^{\mu \nu, d}$, and antisymmetric $\varphi_{2}^{[\mu \nu]}, \varphi_{2}^{[\mu \nu]} \equiv$ $\Psi^{d[\mu, \nu]}$, spin-tensors and one first-rank spin-tensor $\Psi^{\mu}, \Psi^{\mu} \equiv \Psi^{d d, \mu}$. Three final spin-tensors play the role of Stueckelberg fields. The set of gauge parameters (6.111) consists of one second-rank spin-tensor $\xi_{\mu, \nu}$, two first-rank spin-tensors $\xi_{\mu}, \zeta_{\mu} ; \xi_{\mu, d} \equiv \xi_{\mu} ; \xi_{d, \mu} \equiv \zeta_{\mu}$, and one spinor $\xi, \xi \equiv \xi_{d, d}$.

Second, the corresponding action can be obtained from (6.108) by dimensional projection $\mathbb{R}^{1, d} \rightarrow \mathbb{R}^{1, d-1}$, and must be invariant with respect to the gauge transformations

$$
\begin{aligned}
\delta \Psi^{\mu \nu, \rho} & =\frac{\imath}{2} \partial^{\rho} \xi^{\{\nu, \mu\}}-\frac{\imath}{2} \partial^{\{\nu} \xi^{\mu\}, \rho}, & \delta \varphi_{1}^{\mu \nu} & =\frac{m}{2} \xi^{\{\mu, \nu\}}+\frac{\imath}{2} \partial^{\{\nu} \xi^{\mu\}}, \\
\delta \varphi_{2}^{[\mu \nu]} & =-\frac{\imath}{2} \partial^{[\nu}\left(\xi^{\mu]}-2 \zeta^{\mu]}\right)-\frac{m}{2} \xi^{[\mu, \nu]}, & \delta \Psi^{\mu} & =\imath \partial^{\mu} \xi+m \zeta^{\mu}
\end{aligned}
$$

which, in turn, are reducible:

$$
\begin{array}{ll}
\delta \xi_{\mu, \nu}=\imath \partial_{\nu} \xi_{\mu}^{(1)}, & \delta \xi_{\mu}=-m \xi_{\mu}^{(1)} \\
\delta \zeta_{\mu}=\imath \partial_{\mu} \xi_{d}^{(1)}, & \delta \xi=-m \xi_{d}^{(1)}
\end{array}
$$

Third, due to the specific character of the relation for the quantity $\tilde{\gamma} T_{0} \tilde{\gamma}$, identical with $T_{0}$ for massless HS fields, and, when transformed, for massive fields, as $\tilde{\gamma} T_{0} \tilde{\gamma}=T_{0}^{*}, T_{0}^{*}=-\left(\imath \tilde{\gamma}^{\mu} \partial_{\mu}+\tilde{\gamma} m\right)$, with an odd $\tilde{\gamma}$-matrix arising within $\tilde{\gamma}^{\mu}$-matrix. When calculating the scalar products to obtain the final Lagrangian, we use the identification

$$
\begin{aligned}
& i \gamma^{M} \partial_{M} \Psi^{N K, P}=\left(i \gamma^{\mu} \partial_{\mu}-m\right) \Psi^{N K, P}, \quad i \gamma^{M} \partial_{M} \gamma_{N} \Psi^{N K, P}=\left(i \gamma^{\mu} \partial_{\mu}+m\right) \gamma_{N} \Psi^{N K, P} \\
& i \gamma^{M} \partial_{M} \gamma_{N} \gamma_{K} \Psi^{N K, P}=\left(i \gamma^{\mu} \partial_{\mu}-m\right) \gamma_{N} \gamma_{K} \Psi^{N K, P}, \ldots
\end{aligned}
$$

being true, if instead $\Psi^{N K, P}\left[\gamma_{N} \Psi^{N K, P}\right]$ we substitute the quantities $\left(\gamma_{N}\right)^{2 k} \psi^{N K \ldots}\left[\left(\gamma_{N}\right)^{2 k+1} \psi^{N K \ldots]}\right.$, for a non-negative integer $k$.

Then, after removing the gauge parameters $\xi_{\mu}, \xi$, in (6.115) (6.116) by shift transformations with spinors $\xi_{\mu}^{(1)}, \xi_{d}^{(1)}$, respectively, we obtain preliminarily an irreducible gauge theory with independent gauge spin-tensors $\xi_{\mu, \nu}, \zeta_{\mu}$. Next, in the same manner, we can gauge away the 
spin-tensors $\Psi_{\mu}, \varphi_{1}^{\mu \nu}, \varphi_{2}^{[\mu \nu]}$ in Eqs. (6.113), (6.114), by means of gauge transformations with the parameters $\zeta_{\mu}(x), \xi^{\{\mu, \nu\}}$ and $\xi^{[\mu, \nu]}$, respectively, so that the theory becomes a non-gauge one only in terms of a massive 3-rd-rank massive mixed-symmetric spin-tensor $\Psi^{\mu \nu, \rho}$.

Finally, we obtain the Lagrangian for a massive spin-tensor of generalized spin $(5 / 2,3 / 2)$ field in a $d$-dimensional flat space:

$$
\begin{aligned}
\mathcal{L}^{m}\left(\Psi_{\mu \nu, \rho}\right)= & \bar{\Psi}_{\mu \nu, \rho}\left\{\left(\imath \gamma^{\tau} \partial_{\tau}-m\right) \Psi^{\mu \nu, \rho}-\eta^{\mu \nu}\left(\imath \gamma^{\tau} \partial_{\tau}-m\right) \eta^{\sigma \lambda} \Psi_{\sigma \lambda}{ }^{\rho}-\gamma^{\nu} \gamma^{\rho}\left(\imath \gamma^{\tau} \partial_{\tau}\right.\right. \\
& -m) \gamma^{\lambda} \gamma^{\sigma} \Psi_{\sigma, \lambda}^{\mu}-\gamma^{\rho} \gamma^{\nu}\left(\imath \gamma^{\tau} \partial_{\tau}-m\right) \gamma^{\sigma} \gamma^{\lambda} \Psi_{\sigma, \lambda}^{\mu}+2 \gamma^{\rho}\left(\imath \gamma^{\tau} \partial_{\tau}+m\right) \gamma^{\sigma} \Psi^{\mu \nu}{ }_{\sigma} \\
& +4 \gamma^{\nu}\left(\imath \gamma^{\tau} \partial_{\tau}+m\right) \gamma_{\sigma} \Psi^{\mu \sigma, \rho}-4 \imath \partial^{\rho} \gamma^{\sigma} \Psi^{\mu \nu}{ }_{\sigma}-\eta^{\mu \nu} \gamma^{\rho}\left(\imath \gamma^{\tau} \partial_{\tau}+m\right) \eta^{\sigma \lambda} \gamma^{\alpha} \Psi_{\sigma \lambda, \alpha} \\
& -4 \imath \partial^{\{\nu} \gamma^{\sigma} \Psi_{\sigma,}^{\mu}+4 \imath \eta^{\mu \nu}\left(\partial_{\sigma} \gamma^{\lambda} \Psi_{\lambda}^{\sigma, \rho}-\partial^{\rho} \gamma^{\sigma} \eta^{\tau \alpha} \Psi_{\tau \sigma, \alpha}\right)+4 \imath \gamma^{\nu} \gamma^{\rho} \partial_{\sigma} \gamma^{\tau} \Psi_{, \tau}^{\mu \sigma} \\
& \left.+4 \imath \gamma^{\nu} \gamma^{\rho} \partial^{\mu} \eta^{\sigma \lambda} \gamma^{\alpha} \Psi_{\sigma \alpha, \lambda}+4 \imath \gamma^{\rho} \gamma^{\nu} \partial_{\sigma} \gamma^{\alpha} \Psi_{\alpha}{ }^{\mu, \sigma}\right\} .
\end{aligned}
$$

Summarizing, we have obtained a Lagrangian formulation (6.118) only in terms of the initial free massive mixed-symmetric spin-tensor field $\Psi_{\mu \nu, \rho}$. The resulting theory is not a gauge theory, and formula (6.118) presents our basic result of Section 6.4.

\section{Conclusion}

In the present work, we have constructed a gauge-invariant Lagrangian description of free halfinteger HS fields, belonging to an irreducible representation of the Poincare group $I S O(1, d-1)$ with a corresponding Young tableaux having $k$ rows in the "metric-like" formulation. The results of this study are the most general ones and can be applied in the unified way to both massive and massless fermionic HS fields with a mixed symmetry in a Minkowski space of any dimension.

In the standard manner, starting from an embedding of fermionic HS fields into vectors (Dirac spinors) of an auxiliary Fock space, we elaborate the fields as coordinates of Fock-space vectors and reformulate the theory in terms of these objects. The conditions that determine an irreducible Poincare-group representation with a given mass and generalized half-integer spin are realized in terms of differential operator constraints imposed on Fock-space vectors. These constraints generate a closed Lie superalgebra of HS symmetry, which contains, with the exception of $k$ basis generators of its Cartan subalgebra, a system of first- and second-class odd and even constraints. The above superalgebra coincides, modulo the isometry group generators, with its Howe-dual osp $(1 \mid 2 k)$ orthosymplectic superalgebra.

We show that the construction of a correct Lagrangian description requires a deformation of the initial symmetry superalgebra in order to obtain from a system of mixed-class constraints a converted system with the same number of first-class constraints alone, whose structure provides the appearance of the necessary number of auxiliary spin-tensor fields with lower generalized spins within an opposite alphabetic ordering prescription. It is demonstrated that this purpose can be achieved with the help of an additional Fock space, by constructing an additive extension of a symmetry subsuperalgebra, which consists of a subsystem of second-class constraints alone and of the generators of the Cartan subalgebra, which form an invertible even operator supermatrix, composed of supercommutators of the second-class constraints.

The generalized Verma module construction [70], [71] has been realized in order to obtain an auxiliary representation in Fock space for the above superalgebra with second-class constraints. As a consequence, the converted Lie superalgebra of HS symmetry has the same algebraic relations as the initial superalgebra with the only peculiarity that these relations are realized in an enlarged Fock space. The generators of the converted Cartan subalgebra contain linearly $k$ auxiliary independent number parameters $h^{i}$, whose choice provides the vanishing of these generators in 
the corresponding subspaces of the total Hilbert space extended by the ghost operators in accordance with the minimal BFV-BRST construction for the converted HS symmetry superalgebra. Therefore, the above Cartan generators, enlarged by the ghost contributions up to the "particle number" operators in the total Hilbert space, covariantly determine Hilbert subspaces, in each of which the converted symmetry superalgebra consists of the first-class constraints subsystem only. Each of the systems is labelled by the values of the above parameters, and constructed from the initial irreducible Poincare-group relations.

It is demonstrated that the Lagrangian description corresponding to the BRST operator, which encodes the converted HS symmetry superalgebra, yields a consistent Lagrangian dynamics for fermionic fields of any generalized spin after a partial gauge-fixing procedure, that permits one to gauge away the terms with higher (second-order) derivatives from the consideration. The resulting Lagrangian description, realized concisely in terms of the total Fock space, presents a set of generating relations for the action and the sequence of reducible gauge transformations for given fermionic HS fields with a sufficient set of auxiliary fields, and proves to be a reducible gauge theory with a finite number of reducibility stages $s$, increasing with both the value of generalized spin $\left(n_{1}+\frac{1}{2}, \ldots, n_{k}+\frac{1}{2}\right)$ and the number of rows $k$ in the Young tableaux as $s=\sum_{o=1}^{k} n_{o}+k(k-1) / 2-1$. The basic results of the present work are given by relations (5.33), where the action for a field with an arbitrary generalized half-integer spin is constructed, as well as by relations (5.34)-(5.37), where the gauge transformations for the fields are presented, along with the sequence of reducible gauge transformations and gauge parameters.

It has been proved that the solutions of the Lagrangian equations of motion (5.31), (5.32), as a result of a new partial gauge-fixing procedure and a resolution of some of the equations of motion, correspond to the BRST cohomology space with a vanishing ghost number, which is determined only by the relations that extract the fields of an irreducible Poincare-group representation with a given value of half-integer generalized spin. One should notice that the case of totally antisymmetric spin-tensors developed in Ref. [60] is contained in the general Lagrangian formulation for $s_{1}=s_{2}=\ldots=s_{k}=\frac{3}{2}, k=[(d-1) / 2]$.

As examples demonstrating the applicability of the general scheme, it is shown that it contains as a particular case the Lagrangian formulation for a mixed-symmetric spin-tensors subject to a Young tableaux with two rows, first developed in [59], as well as the new unconstrained Lagrangian formulation in (6.30)- (6.34) for mixed-symmetry fermionic HS fields with three groups of symmetric indices subject to a Young tableaux with three rows, obtained in the literature for the first time. We apply the above algorithm to obtain, first, a new gauge-invariant Lagrangian (6.108) and its reducible gauge transformations (6.109) for a massless field of spin $(5 / 2,3 / 2)$, and, second, a new Lagrangian (6.118) for a massive field of spin (5/2,3/2) only in terms of the corresponding initial spin-tensors of the third rank. In principle, these results permit one to enlarge the obtained Lagrangian formulations to those for an HS spin-tensor of spin $\left(\frac{5}{2}, \frac{3}{2}, \ldots, \frac{3}{2}\right)$ characterized by $k$ rows in the corresponding Young tableaux.

Concluding, one should note that there are many ways to extend the results obtained in this paper. We will outline only some of them. First, the development of a Lagrangian construction for bosonic and fermionic fields with an arbitrary index symmetry in AdS space, along the lines of Ref.[58]. Second, the derivation of component Lagrangians for new simple cases. Third, the development of the unconstrained formulation for fermionic fields with an arbitrary Young tableaux similar to the component formulation with the minimal number of auxiliary fields given in [17] for totally symmetric spin-tensor fields which (as shown [17] in the case of bosonic fields) can also be derived from the obtained general Lagrangian formulation by means of a partial gaugefixing procedure. Fourth, the derivation from an unconstrained formulation of a constrained Lagrangian formulation for arbitrary fermionic fields in a flat space-time (as well as those for bosonic mixed-symmetric HS fields starting from the unconstrained formulation in [31]). Fifth, the 
formulation of a diagrammatic technique within the BRST-BFV approach, where the space-time variables $x^{\mu}$ should be considered on equal footing with the total Fock space variables, and all the field-antifield content has to be determined in terms of Fock-space vectors. Finally, a consistent deformation of the latter construction applied to bosonic and fermionic mixed-symmetric HS fields will permit one to construct an interacting theory with mixed-symmetry fermionic HS fields, including the case of curved (AdS) backgrounds, following in part to the way suggested in [67. We are going to develop a research of these problems in our forthcoming work.

\section{Acknowledgements}

The author is grateful to I.L. Buchbinder for numerous stimulating discussions at all stages of the present research. He also thanks Yu.M. Zinoviev and V.A. Krykhtin for discussions concerning the examples, as well as P.M. Lavrov, K. Stepanyantz and V. Gershun for their comments on the conversion procedure and the structure of constraints. He is grateful to M.A. Vasiliev and V. Dobrev for discussions on the difference of generalized Verma modules from the true Verma module, as well as to V. Mazorchuk for remarks on the generalized Verma module structure, to R. Rahman for correspondence, to P. Moshin for careful reading of the text and to V, Tolstoy for the comments on classification of the HS symmetry superalgebra. The author is grateful to the RFBR grant, project No. 12-02-000121, and the grant for LRSS, project No. 224.2012.2, for partial support. The study was supported by The Ministry of education and science of Russian Federation, project 14.B37.21.0774.

\section{Appendix}

\section{A Construction of Additional Parts for $o s p(1 \mid 2 k)$ Superal- gebra}

Here, we describe the method of constructing an auxiliary representation (known by mathematicians as the generalized Verma module [70, [71, see Appendix A.2 for definitions) for the orthosymplectic superalgebra $\operatorname{osp}(1 \mid 2 k)$ with the second-class constraints $\left\{o_{a}^{\prime}, o_{a}^{\prime+}\right\}=\left\{t_{i}^{\prime}, l_{i j}^{\prime}, t^{\prime i j}, t_{i}^{\prime+}\right.$, $\left.l_{i j}^{\prime+}, t_{i j}^{\prime+}\right\}$ and the Cartan subalgebra elements $g_{0}^{\prime i}$, having in mind the identification of $\operatorname{sep}(1 \mid 2 k)$ elements and those of the HS symmetry algebra $\mathcal{A}^{f}\left(Y(k), \mathbb{R}^{1, d-1}\right)$, given by Eqs.(2.33).

Following the Poincare-Birkhoff-Witt theorem, we start with constructing the generalized Verma module based on the Cartan decomposition of $\operatorname{osp}(1 \mid 2 k)(i \leq j, l<m, i, j, l, m=1, \ldots, k)$

$$
\operatorname{osp}(1 \mid 2 k)=\left\{t_{i}^{\prime+}, l_{i j}^{\prime+}, t_{l m}^{\prime+}\right\} \oplus\left\{g_{0}^{\prime i}\right\} \oplus\left\{t_{i}^{\prime}, l_{i j}^{\prime}, t_{l m}^{\prime}\right\} \equiv \mathcal{E}_{k}^{-} \oplus H_{k} \oplus \mathcal{E}_{k}^{+} 15
$$

We emphasize, first, that for the $s p(2 k)$ subalgebra in $\operatorname{osp}(1 \mid 2 k)$ the Verma module $V(\operatorname{sp}(2 k))$ was constructed in Ref. [31], second, in contrast to the case of totally-symmetric fermionic HS fields on $\mathbb{R}^{1, d-1}$, the negative root vectors in $\mathcal{E}_{k}^{-}$do not commute for $k \geq 2$ (see, Refs. [55], [59]). However, we examine the highest-weight representation of the orthosymplectic algebra $\operatorname{osp}(1 \mid 2 k)$ with the highest-weight vector $|0\rangle_{V}, 16$, which should be annihilated by the positive odd and even

\footnotetext{
${ }^{15}$ We may examine $\operatorname{osp}(1 \mid 2 k)$ in the Cartan-Weyl basis for a unified description, however, without loss of generality, the basis elements of the algebra under consideration will be chosen as in Tables 1 , 2

${ }^{16}$ Despite the fact that, in general, the generalized Verma module can be generated by more than one vector from the (non-diagonalizable by $H_{k}$-elements) representation space, we will have a Verma module structure for the superalgebra $\operatorname{osp}(1 \mid 2 k)$, see Appendix $\mathrm{A.2}$
} 
$\operatorname{roots}\left(E^{\alpha_{i}^{0}}, E^{\alpha_{i}^{1}}\right) \in \mathcal{E}_{k}^{+}$, and being a proper one for the Cartan elements $g_{0}^{i}$,

$$
E^{\alpha_{i}^{0}}|0\rangle_{V}=0, \quad E^{\alpha_{i}^{1}}|0\rangle_{V}=0, \quad g_{0}^{i}|0\rangle_{V}=h^{i}|0\rangle_{V}
$$

The general vector of the generalized Verma module $V(\operatorname{osp}(1 \mid 2 k))$, written concisely as $\left|\vec{N}^{f}\right\rangle_{V}=$ $\left|\vec{n}_{l}^{0} ; \vec{N}\right\rangle_{V}$, has - in terms of occupation numbers with the help of the general vector $|\vec{N}\rangle_{V}=$ $\left|\vec{n}_{i j}, \vec{p}_{r s}\right\rangle_{V}$ - the form of the Verma module $V(\operatorname{sp}(2 k))$ [31],

$$
\left|\vec{n}_{l}^{0} ; \vec{N}\right\rangle_{V}=\left|n_{1}^{0}, \ldots, n_{k}^{0} ; n_{11}, \ldots, n_{1 k}, n_{22}, \ldots, n_{2 k}, \ldots, n_{k k} ; p_{12}, \ldots, p_{1 k}, p_{23}, \ldots, p_{2 k}, \ldots, p_{k-1 k}\right\rangle_{V}
$$

where the non-negative integers $n_{l}^{0} \in \mathbb{Z}_{2},\left(n_{i j}, p_{r s}\right) \in \mathbb{N}_{0}$ mean the exponentials of the corresponding negative root vectors $\left(E^{\alpha_{i}^{0}}, E^{\alpha_{i}^{1}}\right)$, determined in a fixed ordering as

$$
\left|\vec{N}^{f}\right\rangle_{V} \equiv \prod_{l=1}^{k}\left(t_{l}^{++}\right)^{n_{l}^{0}} \prod_{i, j=1, i \leq j}^{k}\left(l_{i j}^{\prime+}\right)^{n_{i j}} \prod_{r=1}^{k-1}\left[\prod_{s=r+1}^{k}\left(t_{r s}^{\prime+}\right)^{p_{r s}}\right]|0\rangle_{V} .
$$

The action of the odd negative root vectors $t_{i}^{\prime+}$ and the Cartan generators $g_{0 i}^{\prime}$ on $\left|\vec{N}^{f}\right\rangle_{V}$ can be immediately found (for $\left[\frac{n_{i^{\prime}}^{0}+1}{2}\right]=1(0)$ when $n_{i^{\prime}}^{0}=1(0)$ ) as follows:

$$
\begin{aligned}
t_{i^{\prime}}^{\prime+}\left|\vec{N}^{f}\right\rangle_{V}= & (-1)^{\sum_{l^{\prime}=1}^{i^{\prime}-1} n_{l}^{\prime 0}}\left(1+\left[\frac{n_{i^{\prime}}^{0}+1}{2}\right]\right)\left|\vec{n}_{l}^{0}+\delta_{i^{\prime} l} \bmod 2 ; \vec{n}_{i j}+\delta_{i^{\prime} i^{\prime}, i j}\left[\frac{n_{i^{\prime}}^{0}+1}{2}\right], \vec{p}_{r s}\right\rangle_{V} \\
& +4 \sum_{l^{\prime}=1}^{i^{\prime}-1}(-1)^{\sum_{m=1}^{l^{\prime}-1} n_{m}^{0}} n_{l^{\prime}}^{0}\left|\vec{n}_{l}^{0}-\delta_{l^{\prime} l} ; \vec{n}_{i j}+\delta_{l^{\prime} i^{\prime}, i j}, \vec{p}_{r s}\right\rangle_{V}, \\
g_{0 i}^{\prime}\left|\vec{N}^{f}\right\rangle_{V}= & \left(n_{i}^{0}+2 n_{i i}+\sum_{l \neq i} n_{i l}-\sum_{s>i} p_{i s}+\sum_{r<i} p_{r i}+h^{i}\right)\left|\vec{N}^{f}\right\rangle_{V} .
\end{aligned}
$$

whereas the action of even negative root vectors, $l_{i j}^{\prime+}, t_{r s}^{\prime+}$ on $\left|\vec{N}^{f}\right\rangle_{V}$ has the form

$$
\begin{aligned}
l_{i^{\prime} j^{\prime}}^{\prime+}\left|\vec{N}^{f}\right\rangle_{V}= & \left|\vec{N}^{f}+\delta_{i^{\prime} j^{\prime}, i j}\right\rangle_{V} \\
t_{r^{\prime} s^{\prime}}^{\prime+}\left|\vec{N}^{f}\right\rangle_{V}= & \left|\vec{n}_{l}^{0} ; \vec{n}_{i j}, \vec{p}_{r s}+\delta_{r^{\prime} s^{\prime}, r s}\right\rangle_{V}-\sum_{k^{\prime}=1}^{r^{\prime}-1} p_{k^{\prime} r^{\prime}}\left|\vec{n}_{l}^{0} ; \vec{n}_{i j}, \vec{p}_{r s}-\delta_{k^{\prime} r^{\prime}, r s}+\delta_{k^{\prime} s^{\prime}, r s}\right\rangle_{V} \\
& -\delta_{l r^{\prime}} n_{r^{\prime}}^{0}\left[4 \sum_{n^{\prime}=r^{\prime}+1}^{s^{\prime}-1} n_{n^{\prime}}^{0}(-1)^{n^{\prime}=r^{\prime}+1} n_{k^{\prime}}^{0}\left|\vec{n}_{l}^{0}-\delta_{l r^{\prime}}-\delta_{l n^{\prime}} ; \vec{n}_{i j}+\delta_{n^{\prime} s^{\prime}, i j}, \vec{p}_{r s}\right\rangle_{V}\right. \\
& \left.+(-1)^{k^{\prime}=r^{\prime}+1} n_{k^{\prime}}^{0}\left(1+\left[\frac{n_{s^{\prime}}^{0}+1}{2}\right]\right)\left|\vec{n}_{l}^{0}-\delta_{l r^{\prime}}+\delta_{l s^{\prime}} \bmod 2 ; \vec{n}_{i j}+\delta_{s^{\prime} s^{\prime}, i j}\left[\frac{n_{s^{\prime}}^{0}+1}{2}\right], \vec{p}_{r s}\right\rangle_{V}\right] \\
& -\sum_{k^{\prime}=1}^{k}\left(1+\delta_{k^{\prime} r^{\prime}}\right) n_{r^{\prime} k^{\prime}}\left|\vec{N}^{f}-\delta_{r^{\prime} k^{\prime}, i j}+\delta_{k^{\prime} s^{\prime}, i j}\right\rangle_{V} .
\end{aligned}
$$

Notice that in Eqs.(A.5)-(A.8) we have used such notation, e.g., for the vector $\left|\vec{N}^{f}+\delta_{i^{\prime} j^{\prime}, i j}\right\rangle_{V}$ in the Eq. (A.7), that is subject to definition (A.3), increasing only the coordinate $n_{i j}$ in the vector $\left|\vec{N}^{f}\right\rangle_{V}$, for $i=i^{\prime}, j=j^{\prime}$, by a unit with unchanged values of the remaining ones, whereas the vector $\left|\vec{n}_{l}^{0} ; \vec{n}_{i j}, \vec{p}_{r s}-\delta_{k^{\prime} r^{\prime}, r s}+\delta_{k^{\prime} s^{\prime}, r s}\right\rangle_{V}$ implies increasing the coordinate $p_{r s}$, for $r=k^{\prime}, s=s^{\prime}$, by 
a unit and decreasing by one unit the coordinate $p_{r s}$, for $r=k^{\prime}, s=r^{\prime}$, with unchanged values of the remaining coordinates in $\left|\vec{N}^{f}\right\rangle_{V}$.

Derivation of relations (A.5), (A.6), (A.8) is based on the the algebraic relations for $\operatorname{sesp}(1 \mid 2 k)$ from Tables 1, 2 and the formula for the product of the graded operators $A, B, n \geq 0$,

$$
A B^{n}=\sum_{k=0}^{n}(-1)^{\varepsilon(A) \varepsilon(B)(n-k)} C_{k}^{(s) n} B^{n-k} \operatorname{ad}_{B}^{k} A, \operatorname{ad}_{B}^{k} A=[[\ldots[A, \overbrace{B\}, \ldots\}, B}^{k \text { times }}\},
$$

with $s=\varepsilon(B)$, and the generalized coefficients for a number of graded combinations, $C_{k}^{(s) n}$ (first introduced in [59]; for details, in particular, concerning the odd Pascal triangle, see [63]) coinciding with the standard ones only for the bosonic operator $B: C_{k}^{(0)}{ }_{n}=C_{k}^{n}=\frac{n !}{k !(n-k) !}$. The coefficients are defined recursively, by the relations

$$
\begin{aligned}
& C_{k}^{(s) n+1}=(-1)^{s(n+k+1)} C_{k-1}^{(s) n}+C_{k}^{(s) n}, \quad n, k \geq 0, \\
& C_{0}^{(s) n}=C_{n}^{(s) n}=1, \quad C_{k}^{(s) n}=0, n<k, s=0,1
\end{aligned}
$$

and possess the properties $C_{k}^{(s) n}=C_{n-k}^{(s) n}$. The corresponding values of $C_{k}^{(1) n}$ are given, for $n \geq k$, by the formulae

$$
C_{k}^{(1) n}=\sum_{i_{k}=1}^{n-k+1} \sum_{i_{k-1}=1}^{n-i_{k}-k+2} \cdots \sum_{i_{2}=1}^{n-\sum_{j=3}^{k}} \sum_{i_{1}=1}^{i_{j}-1}(-1)^{k(n+1)+\sum_{j=1}^{[(k+1) / 2]}\left(i_{2 j-1}+1\right)},
$$

which follow by induction. For our purposes, due to $n_{k}^{0}=0,1$ in (A.3), (A.4), it is sufficient to know that $C_{0}^{(1) 0}=C_{0}^{(1) 1}=1$ and $C_{1}^{(1)_{1}^{n_{l}^{0}}}=n_{l}^{0}$. Second, as was shown in [31], Eq. (A.9) permits one to find both the identities

$$
t_{l^{\prime}}^{\prime}\left|\overrightarrow{0}_{l}^{0} ; \overrightarrow{0}_{i j}, \vec{p}_{r s}\right\rangle_{V}=0, \quad l_{i^{\prime} j^{\prime}}^{\prime}\left|\overrightarrow{0}_{l}^{0} ; \overrightarrow{0}_{i j}, \vec{p}_{r s}\right\rangle_{V}=0
$$

and the equation in the action of the positive "mixed-symmetry" root vectors $t_{r^{\prime} s^{\prime}}^{\prime}$ on the vector $\left|\overrightarrow{0}_{l}^{0} ; \overrightarrow{0}_{i j}, \vec{p}_{r s}\right\rangle_{V}$ (due to the non-commutativity of the negative "mixed-symmetry" root vectors $t_{r s}^{\prime+}$ among each other) in the form

$$
\begin{aligned}
& t_{r^{\prime} s^{\prime}}^{\prime}\left|\overrightarrow{0}_{l}^{0} ; \overrightarrow{0}_{i j}, \vec{p}_{r s}\right\rangle_{V}=\left|C_{\vec{p}_{r s}}^{r^{\prime} s^{\prime}}\right\rangle_{V}-\sum_{n^{\prime}=1}^{l^{\prime}-1} p_{n^{\prime} s^{\prime}}\left|\overrightarrow{0}_{l}^{0} ; \overrightarrow{0}_{i j}, \vec{p}_{r s}-\delta_{n^{\prime} s^{\prime}, r s}+\delta_{n^{\prime} r^{\prime}, r s}\right\rangle_{V} \\
& +\sum_{k^{\prime}=r^{\prime}+1}^{s^{\prime}-1} p_{r^{\prime} k^{\prime}}\left[\prod_{l^{\prime}<r^{\prime}, m^{\prime}>l^{\prime}} \prod_{l^{\prime}=r^{\prime}, s^{\prime}>m^{\prime}>l^{\prime}}\left(t_{l^{\prime} m^{\prime}}^{\prime+}\right)^{p_{l^{\prime} m^{\prime}}-\delta_{r^{\prime} k^{\prime}, l^{\prime} m^{\prime}}}\right] t_{k^{\prime} s^{\prime}}^{\prime} \\
& \times \prod_{q^{\prime}=r^{\prime}, t^{\prime} \geq s^{\prime}} \prod_{q^{\prime}>r^{\prime}, t^{\prime}>q^{\prime}}\left(t_{q^{\prime} t^{\prime}}^{\prime+}\right)^{p_{q^{\prime} t^{\prime}}}|0\rangle_{V} \text {, }
\end{aligned}
$$

with the vector $\left|C_{\vec{p}_{r s}}^{r^{\prime} s^{\prime}}\right\rangle_{V}, r^{\prime}<s^{\prime}$ determined as follows:

$$
\begin{aligned}
\left|C_{\vec{p}_{r s}}^{r^{\prime} s^{\prime}}\right\rangle_{V}= & p_{r^{\prime} s^{\prime}}\left(h^{r^{\prime}}-h^{s^{\prime}}-\sum_{k^{\prime}=s^{\prime}+1}^{k}\left(p_{r^{\prime} k^{\prime}}+p_{s^{\prime} k^{\prime}}\right)+\sum_{k^{\prime}=r^{\prime}+1}^{s^{\prime}-1} p_{k^{\prime} s^{\prime}}-p_{r^{\prime} s^{\prime}}+1\right) \times \\
& \times\left|\overrightarrow{0}_{l}^{0} ; \overrightarrow{0}_{i j}, \vec{p}_{r s}-\delta_{r^{\prime} s^{\prime}, r s}\right\rangle_{V}+\sum_{k^{\prime}=s^{\prime}+1}^{k} p_{r^{\prime} k^{\prime}}\left\{\left|\overrightarrow{0}_{l}^{0} ; \overrightarrow{0}_{i j}, \vec{p}_{r s}-\delta_{r^{\prime} k^{\prime}, r s}+\delta_{s^{\prime} k^{\prime}, r s}\right\rangle_{V}\right. \\
& \left.-\sum_{n^{\prime}=1}^{s^{\prime}-1} p_{n^{\prime} s^{\prime}}\left|\overrightarrow{0}_{l}^{0} ; \overrightarrow{0}_{i j}, \vec{p}_{r s}-\delta_{r^{\prime} k^{\prime}, r s}-\delta_{n^{\prime} s^{\prime}, r s}+\delta_{n^{\prime} k^{\prime}, r s}\right\rangle_{V}\right\} .
\end{aligned}
$$


Since the recurrent relation (A.14) has exactly the same form as the one in the case of the symplectic algebra $s p(2 k)$ in Ref. [31], we have used the known solution of (A.14) in the form

$$
\begin{aligned}
& t_{r^{\prime} s^{\prime}}^{\prime}\left|\overrightarrow{0}_{l}^{0} ; \overrightarrow{0}_{i j}, \vec{p}_{r s}\right\rangle_{V}=\sum_{p=0}^{s^{\prime}-r^{\prime}-1} \sum_{k_{1}^{\prime}=r^{\prime}+1}^{s^{\prime}-1} \ldots \sum_{k_{p}^{\prime}=r^{\prime}+p}^{s^{\prime}-1} \prod_{j=1}^{p} p_{k_{j-1}^{\prime} k_{j}^{\prime}}\left|C_{\vec{p}_{r s}-\sum_{j=1}^{p+1} \delta_{k_{j-1}^{\prime} k_{j}^{\prime}, r s}}^{k_{r^{\prime}}}\right\rangle_{V} \\
& -\sum_{n^{\prime}=1}^{r^{\prime}-1} p_{n^{\prime} s^{\prime}}\left|\overrightarrow{0}_{l}^{0} ; \overrightarrow{0}_{i j}, \vec{p}_{r s}-\delta_{n^{\prime} s^{\prime}, r s}+\delta_{n^{\prime} r^{\prime}, r s}\right\rangle_{V}, \quad k_{0}^{\prime} \equiv r^{\prime} .
\end{aligned}
$$

Therefore, the final result for the action of $t_{r^{\prime} s^{\prime}}^{\prime}$ on a vector $\left|\vec{N}^{f}\right\rangle_{V}$ can be written as follows:

$$
\begin{aligned}
& t_{r^{\prime} s^{\prime}}^{\prime}\left|\vec{N}^{f}\right\rangle_{V}=-\sum_{n^{\prime}=1}^{r^{\prime}-1} p_{n^{\prime} s^{\prime}}\left|\vec{n}_{l}^{0} ; \vec{n}_{i j}, \vec{p}_{r s}-\delta_{n^{\prime} s^{\prime}, r s}+\delta_{n^{\prime} r^{\prime}, r s}\right\rangle_{V}
\end{aligned}
$$

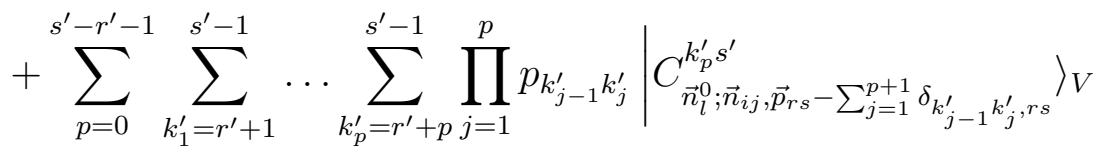

$$
\begin{aligned}
& -\sum_{k^{\prime}=1}^{k}\left(1+\delta_{k^{\prime} s^{\prime}}\right) n_{k^{\prime} s^{\prime}}\left|\vec{n}_{l}^{0} ; \vec{n}_{i j}-\delta_{k^{\prime} s^{\prime}, i j}+\delta_{k^{\prime} r^{\prime}, i j}, \vec{p}_{r s}\right\rangle_{V} \\
& -n_{s^{\prime}}^{0}\left[4 \sum_{k^{\prime}=r^{\prime}+1}^{s^{\prime}-1} n_{k^{\prime}}^{0}(-1)^{n^{\prime}=k^{\prime}+1} n_{n^{\prime}-1}^{0} n_{n^{\prime}}^{0}-\delta_{l k^{\prime}}-\delta_{l s^{\prime}} ; \vec{n}_{i j}+\delta_{k^{\prime} r^{\prime}, i j}, \vec{p}_{r s}\right\rangle_{V} \\
& \left.+(-1)^{k^{\prime}=r^{\prime}+1} n_{k^{\prime}}^{0}\left(1+\left[\frac{n_{r^{\prime}}^{0}+1}{2}\right]\right)\left|\vec{n}_{l}^{0}+\delta_{l r^{\prime}} \bmod 2-\delta_{l s^{\prime}} ; \vec{n}_{i j}+\delta_{r^{\prime} r^{\prime}, i j}\left[\frac{n_{r^{\prime}}^{0}+1}{2}\right], \vec{p}_{r s}\right\rangle_{V}\right] \text {, }
\end{aligned}
$$

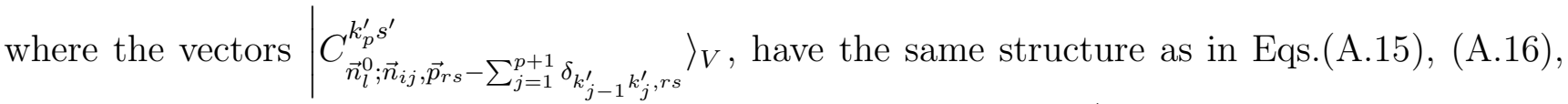
where the only substitution $\left(\overrightarrow{0}_{l}^{0} ; \overrightarrow{0}_{i j}\right) \rightarrow\left(\vec{n}_{l}^{0} ; \vec{n}_{i j}\right)$ should be made in $\left|C_{\vec{p}_{r s}-\sum_{j=1}^{k_{s}^{\prime} \delta_{k_{j-1}^{\prime}} k_{j}^{\prime}, r s}}\right\rangle_{V}$.

Then, it is not difficult to obtain the action of the odd positive-root operators $E^{\prime \alpha^{0}}$ on the vector $\left|\vec{N}^{f}\right\rangle_{V}$ in the form

$$
\begin{aligned}
& t_{i^{\prime}}^{\prime}\left|\vec{N}^{f}\right\rangle_{V}=2 \sum_{k^{\prime}=1}^{i^{\prime}-1} n_{k^{\prime}}^{0}(-1)^{\sum_{l^{\prime}=1}^{k^{\prime}-1} n_{l^{\prime}}^{0}}\left\{-\sum_{n^{\prime}=1}^{k^{\prime}-1} p_{n^{\prime} i^{\prime}}\left|\vec{n}_{l}^{0}-\delta_{k^{\prime} l} ; \vec{n}_{i j}, \vec{p}_{r s}-\delta_{n^{\prime} i^{\prime}, r s}+\delta_{n^{\prime} k^{\prime}, r s}\right\rangle_{V}\right.
\end{aligned}
$$

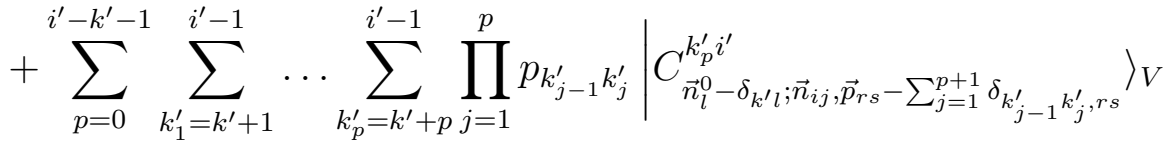

$$
\begin{aligned}
& -\sum_{l^{\prime}=1}^{k}\left(1+\delta_{l^{\prime} i^{\prime}}\right) n_{l^{\prime} i^{\prime}}\left|\vec{n}_{l}^{0}-\delta_{k^{\prime} l} ; \vec{n}_{i j}-\delta_{l^{\prime} i^{\prime}, i j}+\delta_{l^{\prime} k^{\prime}, i j}, \vec{p}_{r s}\right\rangle_{V} \\
& -n_{i^{\prime}}^{0}\left[4 \sum_{n^{\prime}=k^{\prime}+1}^{i^{\prime}-1} n_{n^{\prime}}^{0}(-1)^{m^{\prime}=n^{\prime}+1} \sum_{m^{\prime}}^{\sum^{\prime}-1} n_{n}^{0} n_{l}^{0}-\delta_{l n^{\prime}}-\delta_{l i^{\prime}}-\delta_{l k^{\prime}} ; \vec{n}_{i j}+\delta_{n^{\prime} k^{\prime}, i j}, \vec{p}_{r s}\right\rangle_{V} \\
& \left.\left.+(-1)^{\sum_{m^{\prime}=k^{\prime}+1}^{i^{\prime}-1} n_{m^{\prime}}^{0}}\left|\vec{n}_{l}^{0}-\delta_{l i^{\prime}} ; \vec{n}_{i j}, \vec{p}_{r s}\right\rangle_{V}\right]\right\}
\end{aligned}
$$




$$
\begin{aligned}
& -2 n_{i^{\prime}}^{0}\left(2 n_{i^{\prime} i^{\prime}}+\sum_{l \neq i^{\prime}} n_{i^{\prime} l}-\sum_{s>i^{\prime}} p_{i^{\prime} s}+\sum_{r<i^{\prime}} p_{r i^{\prime}}+h^{i^{\prime}}\right)(-1)^{\sum_{l^{\prime}=1}^{i^{\prime}-1} n_{l^{\prime}}^{0}}\left|\vec{N}^{f}-\delta_{i^{\prime} l}\right\rangle_{V} \\
& +2 \sum_{k^{\prime}=i^{\prime}+1}^{k} n_{k^{\prime}}^{0}(-1) \sum_{l^{\prime}=1}^{k^{\prime}-1} n_{l^{\prime}}^{0}\left\{-\sum_{l^{\prime}=1}^{i^{\prime}-1} p_{l^{\prime} i^{\prime}}\left|\vec{n}_{l}^{0}-\delta_{k^{\prime} l} ; \vec{n}_{i j}, \vec{p}_{r s}-\delta_{l^{\prime} i^{\prime}, r s}+\delta_{l^{\prime} k^{\prime}, r s}\right\rangle_{V}\right. \\
& \left.+\left|\vec{N}^{f}-\delta_{k^{\prime} l}+\delta_{i^{\prime} k^{\prime}, r s}\right\rangle_{V}-\sum_{l^{\prime}=1}^{k}\left(1+\delta_{l^{\prime} i^{\prime}}\right) n_{i^{\prime} l^{\prime}}\left|\vec{N}^{f}-\delta_{k^{\prime} l}-\delta_{i^{\prime} l^{\prime}, i j}+\delta_{k^{\prime} l^{\prime}, i j}\right\rangle_{V}\right\} \\
& -(-1) \sum_{l^{\prime}=1}^{k} n_{l^{\prime}}^{0} \sum_{k^{\prime}=1}^{k}\left(1+\delta_{k^{\prime} i^{\prime}}\right) n_{i^{\prime} k^{\prime}}\left\{2 \sum_{m^{\prime}=k^{\prime}+1}^{k} n_{m^{\prime}}^{0}(-1)^{n^{\prime}=m^{\prime}+1} n_{n^{\prime}}^{k}\left|\overrightarrow{N^{f}}-\delta_{m^{\prime} l}-\delta_{i^{\prime} k^{\prime}, i j}+\delta_{k^{\prime} m^{\prime}, i j}\right\rangle_{V}\right. \\
& \left.+\frac{1}{2}\left(1+\left[\frac{n_{k^{\prime}}^{0}+1}{2}\right]\right)(-1)^{\sum_{n^{\prime}=k^{\prime}+1}^{k} n_{n^{\prime}}^{0}}\left|\vec{N}^{f}+\delta_{k^{\prime} l} \bmod 2-\delta_{i^{\prime} k^{\prime}, i j}+\delta_{k^{\prime} k^{\prime}, i j}\left[\frac{n_{k^{\prime}}^{0}+1}{2}\right]\right\rangle_{V}\right\} \text {, }
\end{aligned}
$$

where we have taken into account the first relations in (A.13). In turn, for the even positive root operators $l_{l^{\prime} m^{\prime}}^{\prime}$, for $l^{\prime}=m^{\prime}$, we have, with allowance for the second relations in (A.13),

$$
\begin{aligned}
& l_{l^{\prime} l^{\prime}}^{\prime}\left|\vec{N}^{f}\right\rangle_{V}=-n_{l^{\prime}}^{0}\left[2 \sum _ { k ^ { \prime } = l ^ { \prime } + 1 } ^ { k } n _ { k ^ { \prime } } ^ { 0 } ( - 1 ) ^ { n ^ { \prime } = l ^ { \prime } + 1 } n _ { n ^ { \prime } } ^ { k ^ { \prime } - 1 } \left\{\left|\vec{n}_{l}^{0}-\delta_{k^{\prime} l}-\delta_{l^{\prime} l} ; \vec{n}_{i j}, \vec{p}_{r s}+\delta_{l^{\prime} k^{\prime}, r s}\right\rangle_{V}\right.\right. \\
& -\sum_{n^{\prime}=1}^{l^{\prime}-1} p_{n^{\prime} l^{\prime}}\left|\vec{n}_{l}^{0}-\delta_{l^{\prime} l}-\delta_{k^{\prime} l} ; \vec{n}_{i j}, \vec{p}_{r s}-\delta_{n^{\prime} l^{\prime}, r s}+\delta_{n^{\prime} k^{\prime}, r s}\right\rangle_{V} \\
& \left.-\sum_{n^{\prime}=1}^{k}\left(1+\delta_{l^{\prime} n^{\prime}}\right) n_{l^{\prime} n^{\prime}}\left|\vec{N}^{f}-\delta_{k^{\prime} l}-\delta_{l^{\prime} l}-\delta_{l^{\prime} n^{\prime}, i j}+\delta_{k^{\prime} n^{\prime}, i j}\right\rangle_{V}\right\} \\
& -(-1)^{\sum_{n^{\prime}=l^{\prime}+1}^{k} n_{n^{\prime}}^{0}} \sum_{k^{\prime}=1}^{k}\left(1+\delta_{k^{\prime} l^{\prime}}\right) n_{l^{\prime} k^{\prime}}\left\{2 \sum_{m^{\prime}=k^{\prime}+1, m^{\prime} \neq l^{\prime}}^{k} n_{m^{\prime}}^{0}(-1)^{\sum_{n^{\prime}=m^{\prime}+1}^{k} n_{n^{\prime}}^{0}}\right. \\
& \times\left|\vec{N}^{f}-\delta_{m^{\prime} l}-\delta_{l^{\prime} l}-\delta_{l^{\prime} k^{\prime}, i j}+\delta_{k^{\prime} m^{\prime}, i j}\right\rangle_{V}+\frac{1}{2}\left(1+\left[\frac{n_{k^{\prime}}^{0}-\delta_{l^{\prime} k^{\prime}}+1}{2}\right]\right)(-1)^{n^{\prime}=k^{\prime}+1} \sum_{n^{\prime}}^{k} n^{0} \\
& \left.\left.\times\left|\overrightarrow{N^{f}}-\delta_{l^{\prime} l}+\delta_{k^{\prime} l} \bmod 2-\delta_{l^{\prime} k^{\prime}, i j}+\delta_{k^{\prime} k^{\prime}, i j}\left[\frac{n_{k^{\prime}}^{0}-\delta_{l^{\prime} k^{\prime}}+1}{2}\right]\right\rangle_{V}\right\}\right]+\left.l_{l^{\prime} l^{\prime}}^{\prime}\left|\overrightarrow{0}_{l}^{0} ; \vec{N}\right\rangle_{V}\right|_{\overrightarrow{0}_{l}^{0} \rightarrow \vec{n}_{l}^{0}},
\end{aligned}
$$

where the quantity $l_{l^{\prime} l^{\prime}}^{\prime}\left|\overrightarrow{0}_{l}^{0} ; \vec{N}\right\rangle_{V}$ denotes the purely symplectic $(s p(2 k))$ part of the action of $l_{l^{\prime} l^{\prime}}^{\prime}$ on $\left|\vec{N}^{f}\right\rangle_{V}$, first derived in Ref. [31], with unchanged values of the "odd" integers $\vec{n}_{l}^{0}$. Explicitly, the expression $l_{l^{\prime} l^{\prime}}^{\prime}\left|\overrightarrow{0}_{l}^{0} ; \vec{N}\right\rangle_{V}$ reads

$$
\begin{aligned}
\left.l_{l^{\prime} l^{\prime}}^{\prime}\left|\overrightarrow{0}_{l}^{0} ; \vec{N}\right\rangle_{V}\right|_{\overrightarrow{0}_{l}^{0} \rightarrow \vec{n}_{l}^{0}}= & -\frac{1}{2} \sum_{k^{\prime}=1}^{l^{\prime}-1} n_{k^{\prime} l^{\prime}}\left[-\sum_{n^{\prime}=1}^{k^{\prime}-1} p_{n^{\prime} l^{\prime}}\left|\vec{n}_{l}^{0} ; \vec{n}_{i j}-\delta_{k^{\prime} l^{\prime}, i j}, \vec{p}_{r s}-\delta_{n^{\prime} l^{\prime}, r s}+\delta_{n^{\prime} k^{\prime}, r s}\right\rangle_{V}\right. \\
& +\sum_{p=0}^{l^{\prime}-k^{\prime}-1} \sum_{k_{1}^{\prime}=k^{\prime}+1}^{l^{\prime}-1} \ldots \sum_{k_{p}^{\prime}=k^{\prime}+p} \prod_{j=1}^{l^{\prime}-1} p_{k_{j-1}^{\prime} k_{j}^{\prime}} \mid C_{\left.\vec{n}_{l}^{0} ; \vec{n}_{i j}-\delta_{k^{\prime} l^{\prime}, i j}, \vec{p}_{r s}-\sum_{j=1}^{k^{\prime} l^{\prime}} \delta_{k_{j-1}^{\prime} k_{j}^{\prime}, r s}\right\rangle_{V}}
\end{aligned}
$$




$$
\begin{aligned}
& \left.-\sum_{n^{\prime}=k^{\prime}+1}^{k}\left(1+\delta_{n^{\prime} l^{\prime}}\right) n_{n^{\prime} l^{\prime}}\left|\vec{n}_{l}^{0} ; \vec{n}_{i j}-\delta_{k^{\prime} l^{\prime}, i j}-\delta_{n^{\prime} l^{\prime}, i j}+\delta_{k^{\prime} n^{\prime}, i j}, \vec{p}_{r s}\right\rangle_{V}\right] \\
& +n_{l^{\prime} l^{\prime}}\left(n_{l^{\prime} l^{\prime}}-1+\sum_{k^{\prime}>l^{\prime}} n_{k^{\prime} l^{\prime}}-\sum_{s>l^{\prime}} p_{l^{\prime} s}+\sum_{r<l^{\prime}} p_{r l^{\prime}}+h^{l^{\prime}}\right)\left|\vec{N}^{f}-\delta_{l^{\prime} l^{\prime}, i j}\right\rangle_{V} \\
& -\frac{1}{2} \sum_{k^{\prime}=l^{\prime}+1}^{k} n_{l^{\prime} k^{\prime}}\left[\left|\vec{n}_{l}^{0} ; \vec{n}_{i j}-\delta_{l^{\prime} k^{\prime}, i j}, \vec{p}_{r s}+\delta_{l^{\prime} k^{\prime}, r s}\right\rangle_{V}\right. \\
& -\sum_{n^{\prime}=1}^{l^{\prime}-1} p_{n^{\prime} l^{\prime}}\left|\vec{n}_{l}^{0} ; \vec{n}_{i j}-\delta_{l^{\prime} k^{\prime}, i j}, \vec{p}_{r s}-\delta_{n^{\prime} l^{\prime}, r s}+\delta_{n^{\prime} k^{\prime}, r s}\right\rangle_{V} \\
& \left.-\sum_{n^{\prime}=k^{\prime}+1}^{k}\left(1+\delta_{n^{\prime} l^{\prime}}\right) n_{n^{\prime} l^{\prime}}\left|\vec{N}^{f}-\delta_{l^{\prime} k^{\prime}, i j}-\delta_{l^{\prime} n^{\prime}, i j}+\delta_{k^{\prime} n^{\prime}, i j}\right\rangle_{V}\right] \\
& +\frac{1}{2} \sum_{k^{\prime}=1, k^{\prime} \neq l^{\prime}}^{k} \frac{n_{l^{\prime} k^{\prime}}\left(n_{l^{\prime} k^{\prime}}-1\right)}{2}\left|\vec{N}^{f}-2 \delta_{l^{\prime} k^{\prime}, i j}+\delta_{k^{\prime} k^{\prime}, i j}\right\rangle_{V} .
\end{aligned}
$$

Finally, for the operators $l_{l^{\prime} m^{\prime}}^{\prime}$, for $l^{\prime}<m^{\prime}$ we have

$$
\begin{aligned}
& l_{l^{\prime} m^{\prime}}^{\prime}\left|\vec{N}^{f}\right\rangle_{V}=-\frac{1}{2} n_{l^{\prime}}^{0}\left[2 \sum _ { k ^ { \prime } = l ^ { \prime } + 1 } ^ { m ^ { \prime } - 1 } n _ { k ^ { \prime } } ^ { 0 } ( - 1 ) ^ { \sum _ { n ^ { \prime } = l ^ { \prime } + 1 } ^ { k ^ { \prime } - 1 } n _ { n ^ { \prime } } ^ { 0 } } \left\{-\sum_{n^{\prime}=1}^{k^{\prime}-1} p_{n^{\prime} m^{\prime}}\left|\vec{N}^{f}-\delta_{l^{\prime} l}-\delta_{k^{\prime} l}-\delta_{n^{\prime} m^{\prime}, r s}+\delta_{n^{\prime} k^{\prime}, r s}\right\rangle_{V}\right.\right. \\
& +\sum_{p=0}^{m^{\prime}-k^{\prime}-1} \sum_{k_{1}^{\prime}=k^{\prime}+1}^{m^{\prime}-1} \ldots \sum_{k_{p}^{\prime}=k^{\prime}+p}^{m^{\prime}-1} \prod_{j=1}^{p} p_{k_{j-1}^{\prime} k_{j}^{\prime}}\left|C_{\vec{n}_{l}^{0}-\delta_{l^{\prime} l}-\delta_{k^{\prime} l} ; \vec{n}_{i j}, \vec{p}_{r s}-\sum_{j=1}^{p+1} \delta_{k_{j-1}^{\prime} k_{j}^{\prime}, r s}}^{k^{\prime}}\right\rangle_{V} \\
& -\sum_{n^{\prime}=1}^{k}\left(1+\delta_{n^{\prime} m^{\prime}}\right) n_{n^{\prime} m^{\prime}}\left|\vec{n}_{l}^{0}-\delta_{l^{\prime} l}-\delta_{k^{\prime} l} ; \vec{n}_{i j}-\delta_{n^{\prime} m^{\prime}, i j}+\delta_{n^{\prime} k^{\prime}, i j}, \vec{p}_{r s}\right\rangle_{V} \\
& -n_{m^{\prime}}^{0}\left(4 \sum_{n^{\prime}=k^{\prime}+1}^{m^{\prime}-1} n_{n^{\prime}}^{0}(-1)^{\sum_{n=n^{\prime}+1}^{m^{\prime}-1} n_{n}^{0}}\left|\vec{n}_{l}^{0}-\delta_{l n^{\prime}}-\delta_{l l^{\prime}}-\delta_{l m^{\prime}}-\delta_{l k^{\prime}} ; \vec{n}_{i j}+\delta_{n^{\prime} k^{\prime}, i j}, \vec{p}_{r s}\right\rangle_{V}\right. \\
& \left.\left.+(-1)^{\sum_{n^{\prime}=k^{\prime}+1}^{m^{\prime}-1} n_{n^{\prime}}^{0}}\left|\vec{n}_{l}^{0}-\delta_{l m^{\prime}}-\delta_{l l^{\prime}} ; \vec{n}_{i j}, \vec{p}_{r s}\right\rangle_{V}\right)\right\} \\
& +2 \sum_{k^{\prime}=m^{\prime}+1}^{k} n_{k^{\prime}}^{0}(-1) \sum_{n^{\prime}=l^{\prime}+1}^{k^{\prime}-1} n_{n^{\prime}}^{0}\left\{\left|\vec{n}_{l}^{0}-\delta_{l^{\prime} l}-\delta_{k^{\prime} l} ; \vec{n}_{i j}, \vec{p}_{r s}+\delta_{m^{\prime} k^{\prime}, r s}\right\rangle_{V}\right. \\
& -\sum_{n^{\prime}=1}^{m^{\prime}-1} p_{n^{\prime} m^{\prime}}\left|\vec{n}_{l}^{0}-\delta_{l^{\prime} l}-\delta_{k^{\prime} l} ; \vec{n}_{i j}, \vec{p}_{r s}-\delta_{n^{\prime} m^{\prime}, r s}+\delta_{n^{\prime} k^{\prime}, r s}\right\rangle_{V} \\
& \left.-\sum_{n^{\prime}=1}^{k}\left(1+\delta_{n^{\prime} m^{\prime}}\right) n_{m^{\prime} n^{\prime}}\left|\vec{N}^{f}-\delta_{l^{\prime} l}-\delta_{k^{\prime} l}-\delta_{m^{\prime} n^{\prime}, i j}+\delta_{k^{\prime} n^{\prime}, i j}\right\rangle_{V}\right\} \\
& -2 n_{m^{\prime}}^{0}(-1)^{\sum_{n^{\prime}=l^{\prime}+1}^{m^{\prime}-1} n_{n^{\prime}}^{0}}\left(\sum_{l=1}^{k}\left(1+\delta_{m^{\prime} l}\right) n_{m^{\prime} l}-\sum_{s>m^{\prime}} p_{m^{\prime} s}+\sum_{r<m^{\prime}} p_{r m^{\prime}}+h^{m^{\prime}}\right)\left|\vec{N}^{f}-\delta_{l^{\prime} l}-\delta_{m^{\prime} l}\right\rangle_{V}
\end{aligned}
$$




$$
\begin{aligned}
& -(-1)^{n^{\prime}=l^{\prime}+1} n_{n^{\prime}}^{k} \sum_{k^{\prime}=1}^{k}\left(1+\delta_{k^{\prime} m^{\prime}}\right) n_{m^{\prime} k^{\prime}}\left\{2 \sum_{n^{\prime}=k^{\prime}+1, n^{\prime} \neq l^{\prime}}^{k} n_{n^{\prime}}^{0}(-1)^{\sum_{p^{\prime}=n^{\prime}+1}^{k} n_{p^{\prime}}^{0}}\right. \\
& \times\left|\vec{N}^{f}-\delta_{l^{\prime} l}-\delta_{n^{\prime} l}-\delta_{m^{\prime} k^{\prime}, i j}+\delta_{k^{\prime} n^{\prime}, i j}\right\rangle_{V}+\frac{1}{2}\left(1+\left[\frac{n_{k^{\prime}}^{0}+1-\delta_{l^{\prime} k^{\prime}}}{2}\right]\right)(-1)^{n^{\prime}=k^{\prime}+1} n_{n^{\prime}}^{k} \\
& \left.\left.\times\left|\vec{N}^{f}-\delta_{l^{\prime} l}+\delta_{k^{\prime} l} \bmod 2-\delta_{m^{\prime} k^{\prime}, i j}+\delta_{k^{\prime} k^{\prime}, i j}\left[\frac{n_{k^{\prime}}^{0}-\delta_{l^{\prime} k^{\prime}}+1}{2}\right]\right\rangle_{V}\right\}\right]
\end{aligned}
$$

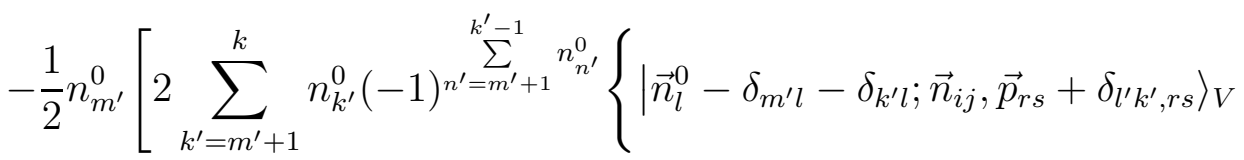

$$
\begin{aligned}
& -\sum_{n^{\prime}=1}^{l^{\prime}-1} p_{n^{\prime} l^{\prime}}\left|\vec{n}_{l}^{0}-\delta_{m^{\prime} l}-\delta_{k^{\prime} l} ; \vec{n}_{i j}, \vec{p}_{r s}-\delta_{n^{\prime} l^{\prime}, r s}+\delta_{n^{\prime} k^{\prime}, r s}\right\rangle_{V} \\
& \left.-\sum_{n^{\prime}=1}^{k}\left(1+\delta_{n^{\prime} l^{\prime}}\right) n_{l^{\prime} n^{\prime}}\left|\vec{N}^{f}-\delta_{m^{\prime} l}-\delta_{k^{\prime} l}-\delta_{l^{\prime} n^{\prime}, i j}+\delta_{k^{\prime} n^{\prime}, i j}\right\rangle_{V}\right\} \\
& -(-1)^{n^{\prime}=m^{\prime}+1} n_{n^{\prime}}^{k} \sum_{k^{\prime}=1}^{k}\left(1+\delta_{l^{\prime} k^{\prime}}\right) n_{l^{\prime} k^{\prime}}\left\{2 \sum_{n^{\prime}=k^{\prime}+1}^{k} n_{n^{\prime}}^{0}(-1)^{\sum_{p^{\prime}=n^{\prime}+1}^{k} n_{p^{\prime}}^{0}}\right. \\
& \times\left|\vec{N}^{f}-\delta_{m^{\prime} l}-\delta_{n^{\prime} l}-\delta_{l^{\prime} k^{\prime}, i j}+\delta_{k^{\prime} n^{\prime}, i j}\right\rangle_{V}+\frac{1}{2}\left(1+\left[\frac{n_{k^{\prime}}^{0}-\delta_{m^{\prime} k^{\prime}}+1}{2}\right]\right)(-1)^{\sum_{n^{\prime}=k^{\prime}+1}^{k} n_{n^{\prime}}^{0}} \\
& \left.\left.\times\left|\vec{N}^{f}-\delta_{m^{\prime} l}+\delta_{k^{\prime} l} \bmod 2-\delta_{l^{\prime} k^{\prime}, i j}+\delta_{k^{\prime} k^{\prime}, i j}\left[\frac{n_{k^{\prime}}^{0}-\delta_{m^{\prime} k^{\prime}}+1}{2}\right]\right\rangle_{V}\right\}\right] \\
& +\left.l_{l^{\prime} m^{\prime}}^{\prime}\left|\overrightarrow{0}_{l}^{0} ; \vec{n}_{i j}, \vec{p}_{r s}\right\rangle_{V}\right|_{\overrightarrow{0}_{l}^{0} \rightarrow \vec{n}_{l}^{0}}
\end{aligned}
$$

where, in order to obtain Eq.(A.21), the formulae for the purely symplectic $(s p(2 k))$ part of the action of $l_{l^{\prime} l^{\prime}}^{\prime}$ on $\left|\overrightarrow{N^{f}}\right\rangle_{V}$, i.e., $l_{l^{\prime} l^{\prime}}^{\prime}\left|\overrightarrow{0}_{l}^{0} ; \vec{N}\right\rangle_{V}$, first derived in Ref. [31], with unchanged values of "odd" integers $\vec{n}_{l}^{0}$, have been written. Explicitly, we have

$$
\begin{aligned}
\left.l_{l^{\prime} m^{\prime}}^{\prime}\left|\overrightarrow{0}_{l}^{0} ; \vec{N}\right\rangle_{V}\right|_{\overrightarrow{0}_{l}^{0} \rightarrow \vec{n}_{l}^{0}=}= & \frac{1}{4} \sum_{k^{\prime}=1}^{m^{\prime}-1}\left(1+\delta_{k^{\prime} l^{\prime}}\right) n_{k^{\prime} l^{\prime}}\left[-\sum_{n^{\prime}=1}^{k^{\prime}-1} p_{n^{\prime} m^{\prime}}\left|\vec{N}^{f}-\delta_{k^{\prime} l^{\prime}, i j}-\delta_{n^{\prime} m^{\prime}, r s}+\delta_{n^{\prime} k^{\prime}, r s}\right\rangle_{V}(A\right. \\
& \left.+\sum_{p=0}^{m^{\prime}-k^{\prime}-1} \sum_{k_{1}^{\prime}=k^{\prime}+1}^{m^{\prime}-1} \cdots \sum_{k_{p}^{\prime}=k^{\prime}+p}^{m^{\prime}-1} \prod_{j=1}^{p} p_{k_{j-1}^{\prime} k_{j}^{\prime}} \mid C_{\left.\vec{n}_{l}^{0} ; \vec{n}_{i j}-\delta_{k^{\prime} l^{\prime}, i j}, \vec{p}_{r s}-\sum_{j=1}^{p+1} \delta_{k_{j-1}^{\prime} k_{j}^{\prime}, r s}\right\rangle_{V}}^{k^{\prime}}\right] \\
& \left.-\sum_{n^{\prime}=k^{\prime}}^{k}\left(1+\delta_{n^{\prime} m^{\prime}}\right) n_{n^{\prime} m^{\prime}}\left|\vec{n}_{l}^{0} ; \vec{n}_{i j}-\delta_{k^{\prime} l^{\prime}, i j}-\delta_{n^{\prime} m^{\prime}, i j}+\delta_{k^{\prime} n^{\prime}, i j}, \vec{p}_{r s}\right\rangle_{V}\right] \\
& -\frac{1}{4} \sum_{k^{\prime}=m^{\prime}+1}^{k} n_{l^{\prime} k^{\prime}}\left[\left|\vec{n}_{l}^{0} ; \vec{n}_{i j}-\delta_{l^{\prime} k^{\prime}, i j}, \vec{p}_{r s}+\delta_{m^{\prime} k^{\prime}, r s}\right\rangle_{V}\right. \\
& -\sum_{n^{\prime}=1}^{m^{\prime}-1} p_{n^{\prime} m^{\prime}}\left|\vec{n}_{l}^{0} ; \vec{n}_{i j}-\delta_{l^{\prime} k^{\prime}, i j}, \vec{p}_{r s}-\delta_{n^{\prime} m^{\prime}, r s}+\delta_{n^{\prime} k^{\prime}, r s}\right\rangle_{V} \\
& \left.-\sum_{n^{\prime}=l^{\prime}+1}^{k}\left(1+\delta_{n^{\prime} m^{\prime}}\right) n_{m^{\prime} n^{\prime}}\left|\vec{n}_{l}^{0} ; \vec{n}_{i j}-\delta_{l^{\prime} k^{\prime}, i j}-\delta_{n^{\prime} m^{\prime}, i j}+\delta_{k^{\prime} n^{\prime}, i j}\right\rangle_{V}\right]
\end{aligned}
$$




$$
\begin{aligned}
& +\frac{1}{4} n_{l^{\prime} m^{\prime}}\left(n_{l^{\prime} m^{\prime}}-1+\sum_{k^{\prime}>l^{\prime}}\left(1+\delta_{k^{\prime} m^{\prime}}\right) n_{k^{\prime} m^{\prime}}+\sum_{k^{\prime}>m^{\prime}} n_{l^{\prime} k^{\prime}}-\sum_{s>l^{\prime}} p_{l^{\prime} s}\right. \\
& \left.-\sum_{s>m^{\prime}} p_{m^{\prime} s}+\sum_{r<l^{\prime}} p_{r l^{\prime}}+\sum_{r<m^{\prime}} p_{r m^{\prime}}+h^{l^{\prime}}+h^{m^{\prime}}\right)\left|\vec{n}_{l}^{0} ; \vec{n}_{i j}-\delta_{l^{\prime} m^{\prime}, i j}, \vec{p}_{r s}\right\rangle_{V} \\
& -\frac{1}{4} \sum_{k^{\prime}=1}^{l^{\prime}-1} n_{k^{\prime} m^{\prime}}\left[-\sum_{n^{\prime}=1}^{k^{\prime}-1} p_{n^{\prime} l^{\prime}}\left|\vec{n}_{l}^{0} ; \vec{n}_{i j}-\delta_{k^{\prime} m^{\prime}, i j}, \vec{p}_{r s}-\delta_{n^{\prime} l^{\prime}, r s}+\delta_{n^{\prime} k^{\prime}, r s}\right\rangle_{V}\right.
\end{aligned}
$$

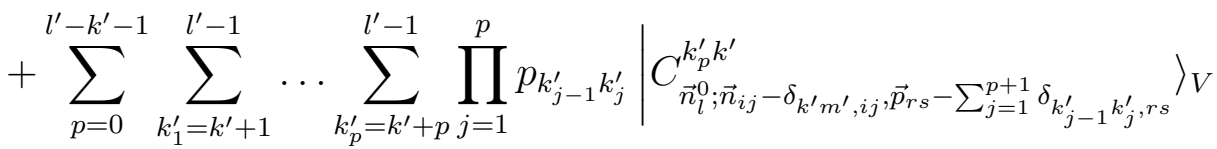

$$
\begin{aligned}
& \left.-\sum_{n^{\prime}=k^{\prime}+1}^{k}\left(1+\delta_{n^{\prime} l^{\prime}}\right) n_{n^{\prime} l^{\prime}}\left|\vec{n}_{l}^{0} ; \vec{n}_{i j}-\delta_{k^{\prime} m^{\prime}, i j}-\delta_{n^{\prime} l^{\prime}, i j}+\delta_{k^{\prime} n^{\prime}, i j}, \vec{p}_{r s}\right\rangle_{V}\right] \\
& -\frac{1}{4} \sum_{k^{\prime}=l^{\prime}+1}^{k}\left(1+\delta_{k^{\prime} m^{\prime}}\right) n_{m^{\prime} k^{\prime}}\left[\left|\vec{n}_{l}^{0} ; \vec{n}_{i j}-\delta_{m^{\prime} k^{\prime}, i j}, \vec{p}_{r s}+\delta_{l^{\prime} k^{\prime}, r s}\right\rangle_{V}\right. \\
& \left.-\sum_{n^{\prime}=1}^{l^{\prime}-1} p_{n^{\prime} l^{\prime}}\left|\vec{n}_{l}^{0} ; \vec{n}_{i j}-\delta_{m^{\prime} k^{\prime}, i j}, \vec{p}_{r s}-\delta_{n^{\prime} l^{\prime}, r s}+\delta_{n^{\prime} k^{\prime}, r s}\right\rangle_{V}\right] .
\end{aligned}
$$

Thus, formulae (A.5) - (A.8), (A.17) - (A.22) completely solve the problem of an auxiliary representation (generalized Verma module) for the orthosymplectic $\operatorname{osp}(1 \mid 2 k)$ algebra. Notice that the above result contains, as a particular case, for $\vec{n}_{l}^{0}=\overrightarrow{0}_{l}^{0}$, and without the odd root vectors $t_{i}^{\prime}, t_{i}^{\prime+}$, the Verma module for the symplectic $s p(2 k)$ algebra constructed in Ref.[31].

\section{A.1 On Construction of Additional Parts for Massive Half-integer HS Fields}

The solution of a similar problem of constructing the auxiliary representation for the HS symmetry massive superalgebra $\mathcal{A}^{f}\left(Y(k), \mathbb{R}^{1, d-1}\right)$ is provided by an enlargement of the Cartan decomposition (A.1) for $\operatorname{osp}(1 \mid 2 k)$ up to the one for $\mathcal{A}^{f}\left(Y(k), \mathbb{R}^{1, d-1}\right)$. Then, we can make the same steps again, adding, first, the "divergence", $l_{i}^{\prime}$, and "gradient", $l_{i}^{\prime+}$, operators, respectively, to the subsuperalgebras of positive $\mathcal{E}_{k}^{+}$and negative $\mathcal{E}_{k}^{-}$root vectors in (A.1), and, second, with the peculiarity, that the Cartan-like subsuperalgebra would now contain two elements $t_{0}^{\prime} ; l_{0}^{\prime 17}$. Simultaneously, the highest-weight vector $|0\rangle_{V}$ and the basis vector $\left|\vec{N}_{m}^{f}\right\rangle_{V}$ of $\mathcal{A}^{f}\left(Y(k), \mathbb{R}^{1, d-1}\right)$, in addition to definitions (A.2)-(A.4), should be determined as follows:

$$
\begin{aligned}
l_{i}^{\prime}|0\rangle_{V} & =0, & t_{0}^{\prime}|0\rangle_{V}=\tilde{\gamma} m|0\rangle_{V}, \\
\left|\vec{N}_{m}^{f}\right\rangle_{V} & \sim \prod_{i}^{k}\left(\frac{l_{i}^{\prime+}}{m_{i}}\right)^{n_{i}}\left|\vec{N}^{f}\right\rangle_{V}, &
\end{aligned}
$$

for some parameters $m_{i} \in \mathbb{R}_{+}$of the dimension of mass, the odd matrix $\tilde{\gamma}$ is from the set of odd gamma-like matrices (2.11), so that the central charge $m$ in the initial algebra $\mathcal{A}^{f}\left(Y(k), \mathbb{R}^{1, d-1}\right)$ vanishes in the converted algebra $\mathcal{A}_{c}^{f}\left(Y(k), \mathbb{R}^{1, d-1}\right)$, because of the additive composition law

$$
m \rightarrow M=m+m^{\prime}=0, \quad\left(t_{0}^{\prime} ; l_{0}\right) \rightarrow\left(T_{0} ; L_{0}\right)=\left(t_{0}+t_{0}^{\prime}=t_{0}+\tilde{\gamma} m ; \quad l_{0}+l_{0}^{\prime}=l_{0}+m^{2}\right),
$$

\footnotetext{
${ }^{17}$ Despite the fact that the anticommutators $\left\{t_{0}^{\prime}, t_{i}^{\prime}\right\},\left\{t_{0}^{\prime}, t_{i}^{\prime+}\right\}$ are not proportional to $t_{i}^{\prime}, t_{i}^{\prime+}$, respectively.
} 
for the central elements $m, m^{\prime}$, we have the respective odd Cartan-like operators $t_{0}, t_{0}^{\prime}$, of the original superalgebra of $o_{I}$ and the superalgebra of additional parts $o_{I}^{\prime}$, with the following relation to the corresponding Casimir operators $l_{0}, l_{0}^{\prime}: t_{0}^{2}=-l_{0} t_{0}^{\prime 2}=-l_{0}^{\prime}$.

\section{A.2 on Verma modules}

The corresponding construction was suggested by the Indian mathematician Daya-Nand Verma in his Ph.D. thesis [68] at the 60 -s of the last century. Let $\mathcal{F}$ be a field (i.g., real $\mathbb{R}$ or complex $\mathbb{C}$ ) and let us consider a semisimple Lie algebra $g$ over $\mathcal{F}$ with the universal enveloping algebra $\mathcal{U}(g)$ and with the Cartan (triangular) decomposition

$$
g=g^{-} \oplus \mathcal{H} \oplus g^{+}, \text {where }[\mathcal{H}, \mathcal{H}]=0,\left[g^{-}, \mathcal{H}\right] \subset g^{-},\left[g^{+}, \mathcal{H}\right] \subset g^{+},
$$

with the Cartan subalgebra $\mathcal{H}$ and the nilpotent subalgebras $g^{-}, g^{+}$, which, within a matrix realization of $g$, are associated with the vector spaces of diagonal matrices and of upper- $g^{-}$and lower- $g^{+}$triangular matrices. The direct sum of $\mathcal{H}$ and $g^{+}$subalgebras, $\mathcal{B}, \mathcal{B}=\mathcal{H} \oplus g^{+}$is called the Borel subalgebra of $g$ and is the maximally solvable subalgebra in it. Then, let $\lambda \in \mathcal{H}^{*}$ be a fixed weight from the (dual to $g$ ) algebra $g^{*}$.

The definition of the Verma module implies the natural presence of some other modules. Let $\mathcal{F}_{\lambda}$ be a one-dimensional vector space over $\mathcal{F}$ together with a $\mathcal{B}$-module structure, being such that

$$
\mathcal{H} \mathcal{F}_{\lambda}=\lambda \mathcal{F}_{\lambda}, \quad g^{+} \mathcal{F}_{\lambda}=0
$$

For any Lie algebra with a triangular decomposition (A.26), the following decomposition for the corresponding universal enveloping algebras $\mathcal{U}(g), \mathcal{U}\left(g^{-}\right), \mathcal{U}(\mathcal{H}), \mathcal{U}\left(g^{+}\right), \mathcal{U}(\mathcal{B})$ is valid:

$$
\mathcal{U}(g)=\mathcal{U}\left(g^{-}\right) \otimes \mathcal{U}(\mathcal{H}) \otimes \mathcal{U}\left(g^{+}\right)=\mathcal{U}\left(g^{-}\right) \otimes \mathcal{U}(\mathcal{B})
$$

Since the set $\mathcal{F}_{\lambda}$ is a left $\mathcal{B}$-module, it can be presented as a left $\mathcal{U}(\mathcal{B})$-module, as well.

An application of the Poincare-Birkhoff-Witt theorem concerning the structure of the basis elements in $\mathcal{U}(g)$ provides a natural right $\mathcal{U}(\mathcal{B})$-module structure on the algebra $\mathcal{U}(g)$ by means of the right multiplication of the Borel subalgebra $\mathcal{B}$. In addition, $\mathcal{U}(g)$ is a natural left $g$-module. Therefore, the universal enveloping algebra $\mathcal{U}(g)$ is a $(g, \mathcal{U}(\mathcal{B})$-bimodule,

$$
\forall a \in \mathcal{U}(g), b \in \mathcal{U}(\mathcal{B}), c \in g: \quad c \otimes a \otimes b \in \mathcal{U}(g)
$$

The Verma module denoted as $M_{\lambda}$ (with respect to the weight $\lambda$ ) is the induced $g$-module, determined by the formula (see, for instance, [73])

$$
M_{\lambda}=\mathcal{U}(g) \otimes_{\mathcal{U}(\mathcal{B})} \mathcal{F}_{\lambda}:=\left(\mathcal{U}(g) \otimes_{\mathcal{F}} \mathcal{F}_{\lambda}\right) / \sum_{a, b, v} \mathcal{F}(a b \otimes v-a \otimes b(v)),
$$

where the sum runs over all $a \in \mathcal{U}(g), b \in \mathcal{U}(\mathcal{B}), v \in \mathcal{F}_{\lambda}$ and the elements $a b, b(v)$ belong to $\mathcal{U}(g)$ and $\mathcal{F}_{\lambda}$, respectively.

The structure of the Verma module $M_{\lambda}$ for the algebra $g$ for a given weight $\lambda$, which is an infinite-dimensional representation of $g$, is simplified due to the mentioned Poincare-BirkhoffWitt theorem (see, e.g., [68], 69], [70]). Indeed, the underlying vector space (representation space) of the Verma module is isomorphic to $\mathcal{U}\left(g^{-}\right) \otimes_{\mathcal{F}} \mathcal{F}_{\lambda}$,

$$
M_{\lambda}=\mathcal{U}\left(g^{-}\right) \otimes_{\mathcal{F}} \mathcal{F}_{\lambda}
$$

with a nilpotent Lie subalgebra $g^{-}$generated by the negative root spaces of $g$. 
Verma modules can be equivalently determined (it can be regarded a property if the definition (A.30) is a starting point of Verma module introduction) via the notion of $\mathcal{H}$-diagonalizable $g$ module $V$. Recalling that it possesses the last property if it has the decomposition

$$
V=\bigoplus_{\lambda \in \mathcal{H}^{*}} V_{\lambda}, \text { where } V_{\lambda}=\{v \in V \mid h(v)=\langle\lambda, h\rangle v, \text { for } h \in \mathcal{H}\}
$$

on the weight subspaces $V_{\lambda}$ with the non-vanishing vector $v, v \in V_{\lambda}$, called the weight-vector of the weight $\lambda$. Then, let $P(V)=\left\{\lambda \in \mathcal{H}^{*} \mid V_{\lambda} \neq 0\right\}$ denote the set of weights for the module $V$. For $\lambda \in \mathcal{H}^{*}$, we set $D(\lambda)=\left\{\mu \in \mathcal{H}^{*} \mid \mu \leq \lambda\right\}$. Then, the category $\mathcal{O}$, whose objects are $g$-modules $V$, being $\mathcal{H}$-diagonalizable with the corresponding weight subspaces being finite-dimensional and possessing the property of the existence of finite weights $\lambda_{1}, \ldots, \lambda_{s} \in \mathcal{H}^{*}$, so that

$$
P(V) \subset \bigcup_{j=1}^{s} D\left(\lambda_{j}\right) .
$$

The morphisms in $\mathcal{O}$ are homomorphisms of $g$-modules.

Notice that any submodule, quotient module, sum or tensor product of a finite number of modules, from $\mathcal{O}$ belong to $\mathcal{O}$, as well. Let us examine the example of highest-weight modules from the category $\mathcal{O}$. The module $V$ over a Lie algebra $g$ with the highest weight $\lambda \in \mathcal{H}^{*}$ is determined by non-zero vector $v_{\lambda} \in V$, such that

$$
g^{+}\left(v_{\lambda}\right)=0, \quad h\left(v_{\lambda}\right)=\lambda(h) v_{\lambda} \text { for } h \in \mathcal{H} \text { and } \mathcal{U}(g)\left(v_{\lambda}\right)=V
$$

The vector $v_{\lambda}$ is called highest-weight vector. The final condition due to the decomposition (A.28) can be replaced by $\mathcal{U}\left(g^{-}\right)\left(v_{\lambda}\right)=V$.

From Eq. (A.34), it follows that

$$
V=\sum_{\mu \leq \lambda} V_{\mu}, \quad V_{\lambda}=\mathcal{F} v_{\lambda}, \operatorname{dim} V_{\lambda}<\infty
$$

Therefore, the highest-weight module belongs to $\mathcal{O}$, and any highest-weight vectors are proportional.

Now, we can determine the Verma module in a way different than by (A.30). Namely, the highest-weight module $M_{\lambda}$ over a Lie algebra $g$ with the highest weight $\lambda$ is called Verma module if any $g$-module $N_{\lambda}$ with the highest weight $\lambda$ is a quotient module of the module $M_{\lambda}$, i.e., $N_{\lambda}=M_{\lambda} / R_{\lambda}$ for any submodule $R_{\lambda} \subset M_{\lambda}$. The following properties of the Verma module hold true [68, [70]:

a) for any highest weight $\lambda \in \mathcal{H}^{*}$ there exists a module $M_{\lambda}$, unique with accuracy up to a Verma isomorphism;

b) $M_{\lambda}$ as a $\mathcal{U}\left(g^{-}\right)$-module is free of a rank-1 module generated by the highest-weight vector;

c) $M_{\lambda}$ contains a unique proper maximal submodule $M_{\lambda}^{\prime}$.

Notice that the last property implies the existence of a unique irreducible highest-weight module $L_{\lambda}=M_{\lambda} / M_{\lambda}^{\prime}$.

Now, we shortly describe an extension of the Verma module concept known as the generalized Verma module (GVM) [71]. For its introduction, with a given Lie algebra $g$, we consider, instead 
of the Borel subalgebra, some of its parabolic subalgebras $\mathfrak{p}$, where, by the definition of "parabolicity", $\mathfrak{p} \supset \mathcal{B}$. For any irreducible finite dimensional representation space $V$ of $\mathfrak{p}$ the generalized Verma module is determined with the help of induced module terms such as

$$
M_{\mathfrak{p}}(V):=\mathcal{U}(g) \otimes_{\mathcal{U}(\mathfrak{p})} V
$$

In case $\lambda$ is the highest weight of $V$, the GVM may be denoted as $M_{\mathfrak{p}}(\lambda)$, and it makes sense only for a so-called integral and dominant weight $\lambda$ in $\mathfrak{p}$. It is well known that a parabolic subalgebra $\mathfrak{p} \subset g$ determines a unique grading decomposition, $g=\bigoplus_{i=-m}^{m} g_{i}$, in such a way that $\mathfrak{p}=\bigoplus_{i \geq 0}^{m} g_{i}$. Denoting $g^{-}=\bigoplus_{i<0}^{m} g_{i}$, we deduce from the Poincare-Birkhoff-Witt theorem a relation for the representation space of the algebra $g$ GVM:

$$
M_{\mathfrak{p}}(V) \simeq \mathcal{U}\left(g^{-}\right) \otimes_{\mathcal{F}} V
$$

Among the properties of GVM we list the following: first, GVM is the highest-weight module, because the highest weight $\lambda$ of the representation space $V$ is the highest weight of $M_{\mathfrak{p}}(\lambda)$; second, GVMs belong to the category $\mathcal{O}$ of highest-weight modules, and therefore they are quotients of the corresponding Verma module $M_{\lambda}$; third, the kernel $K_{\lambda}$ of the projection $M_{\lambda} \rightarrow M_{\mathfrak{p}}(\lambda)$ forms a (not direct) sum:

$$
K_{\lambda}:=\sum_{\alpha \in S} M_{s_{\alpha} \cdot \lambda} \subset M_{\lambda}
$$

The set $S$ from the set $\Delta$ of all simple roots $\alpha$ of the algebra $g$ is composed from such $\alpha \in S$ that the negative root space $E^{-\alpha}$ of a root $(-\alpha)$ belongs to a subalgebra $\mathfrak{p}$, i.e., $E^{-\alpha} \subset \mathfrak{p}$, and, therefore, a basis of GVM is smaller than that in the Verma module $M_{\lambda}$. Notice that the set $S$ is uniquely determined by $\mathfrak{p}$, and $s_{\alpha}$ is the root reflection with respect to the root $\alpha$ and $s_{\alpha} \cdot \lambda$ represents the affine action of $s_{\alpha}$ on a highest-weight $\lambda$.

In the case of a trivial set $S, S=\emptyset$, the parabolic subalgebra $\mathfrak{p}$ coincides with the Borel subalgebra $\mathcal{B}$ and GVM $M_{\mathfrak{p}}(\lambda)=M_{\lambda}$. In the opposite case, when $S=\Delta$, and therefore $\mathfrak{p}$ coincides with the semi-simple algebra Lie $g$, the GVM is isomorphic to the induced representation $V$.

A consideration of the Verma module and generalized Verma module concepts in the case of a Lie superalgebra $g$ is slightly modified because of the existence of a natural $\mathbb{Z}_{2}$-grading on $g$, but it can be formulated straightforwardly (see, e.g., [74] for algorithms of constructing a firstorder realization for a Lie (super)algebra), as well as an extension of those concepts to the case (super)algebras of more general than semi-simple ones.

To illustrate the applicability of the general constructions, let us examine a semi-simple Lie algebra $g$ with a triangular decomposition (A.26) and Cartan-Weyl basis elements $E^{-\alpha_{1}}, \ldots, E^{-\alpha_{k}} \in$ $g^{-}, H^{i} \in \mathcal{H}, E^{\alpha_{1}}, \ldots, E^{\alpha_{k}} \in g^{+}$, for positive roots $\alpha_{1}, \ldots, \alpha_{k}$, and for $i=1, \ldots$, rank $g$. The independent commutation relations of the basis elements have the form

$$
\left[H^{i}, E^{\alpha}\right]=\alpha_{i} E^{\alpha}, \quad\left[E^{-\alpha}, E^{\alpha}\right]=\sum_{i} \alpha^{i} H^{i}, \quad\left[E^{\alpha}, E^{\beta}\right]=N^{\alpha+\beta} E^{\alpha+\beta}
$$

for $\alpha+\beta \neq 0$, some numbers $\alpha^{i} \in \mathcal{F}$. The corresponding Borel subalgebra of $g$ contains all positive root vectors $E^{\alpha}$ and basis of the Cartan subalgebra $H^{i}$, whereas the basis of the corresponding Verma module $M_{\lambda}$ for the highest weight $\lambda \in \mathcal{H}^{*}$ in some $g$-module $V$ with the highest-weight vector $|0\rangle_{V},|0\rangle_{V} \equiv v_{\lambda}$, determined in accordance with the representation (A.31), (A.34), has the form

$$
M_{\lambda}=\left\{\prod_{j=1}^{k}\left(E^{-\alpha_{j}}\right)^{n_{j}}|0\rangle_{V}\right\}, \quad E^{\alpha_{j}}|0\rangle_{V}=0, \quad H^{i}|0\rangle_{V}=h^{i}|0\rangle_{V}
$$


for $j=1, \ldots, k$ and $\lambda\left(H^{i}\right)=h^{i}$. Such a realization of the Verma module for a real simple Lie algebra $g$ and its oscillator realization was first examined in [72].

The corresponding representation was used (with a choice of a slightly different basis than the Cartan-Weyl basis) both in our previous paper [31] to construct the Verma module and an auxiliary oscillator representation for a semi-simple Lie algebra with second-class constraints $s p(2 k)$ and in Appendix $\mathrm{A}$ for the case of $\operatorname{ssp}(1 \mid 2 k)$ Lie superalgebra, as well as in the papers [47], [64], [55], [59]. The generalized Verma module structure can be realized for a Lie superalgebra which incorporates the odd isometry group element $t_{0}=-\imath \tilde{\gamma}^{\mu} \partial_{\mu}$ for massive half-integer HS fields in Minkowski space, see Appendix A.1 (with $t_{0}=-\imath \tilde{\gamma}^{\mu} D_{\mu}$, for massive half-integer HS fields in AdS space it has been done in [63], [56]) because its odd element from the Cartan subsuperalgebra of the HS symmetry superalgebra $\mathcal{A}^{f}\left(Y(k), \mathbb{R}^{1, d-1}\right)$ (for a quadratic HS symmetry superalgebra $\mathcal{A}^{f}\left(Y(1), A d S_{d}\right)$ ) will not diagonalize the representation space $V$. The same situation with GVM was realized in the case of massive bosonic HS fields in an AdS space both for totallysymmetric [57] and mixed-symmetric with $Y\left(s_{1}, s_{2}\right)$ in [58], where the bosonic element $l_{0}^{\prime}$ from the corresponding Cartan subalgebras does not diagonalize the respective representation space $V$ due to non-trivial AdS-radius presence.

Another example of GVM, can be found if one adds to the Borel subsuperalgebra $\mathcal{B}=H_{k} \oplus$ $\mathcal{E}_{k}^{+}$, from the decomposition of $\operatorname{osp}(1 \mid 2 k)$ given by Eq. (A.1), the negative root vectors from $\mathcal{E}_{k}^{-}$, which contain the index $k$, i.e., the nilpotent subsuperalgebra $\left[\mathcal{E}_{k}^{-}\right]=\left\{t_{k}^{\prime}, l_{i k}^{\prime}, t^{\prime r k}\right\}$ for $i=$ $1, \ldots, k ; r=1, \ldots, k-1$. In this case, the parabolic subsuperalgebra $\mathfrak{p}$ of $\operatorname{osp}(1 \mid 2 k)$ has the form $\mathfrak{p}=\left[\mathcal{E}_{k}^{-}\right] \oplus H_{k} \oplus \mathcal{E}_{k}^{+}$. The basis $\left\{\left|\vec{N}_{g}\right\rangle_{V}\right\}$ of the representation space $V$ with highest-weight vector $\left|0_{g}\right\rangle_{V}$ for GVM, $M_{\mathfrak{p}}(V)$, in accordance with the structure of the $g^{-}$nilpotent subsuperalgebra in the decomposition $\operatorname{osp}(1 \mid 2 k)=g^{-} \oplus \mathfrak{p}: \mathcal{E}_{k}^{+}\left|0_{g}\right\rangle_{V}=\left[\mathcal{E}_{k}^{-}\right]\left|0_{g}\right\rangle_{V}=0, H^{i}\left|0_{g}\right\rangle_{V}=h^{i}\left|0_{g}\right\rangle_{V}$, for $H^{i} \in H_{k}$, with allowance for the representation (A.37), can be presented as

$$
\left|\vec{N}_{g}\right\rangle_{V} \equiv \prod_{l=1}^{k-1}\left(t_{l}^{\prime+}\right)^{n_{l}^{0}} \prod_{i, j=1, i \leq j}^{k-1}\left(l_{i j}^{\prime+}\right)^{n_{i j}} \prod_{r=1}^{k-2}\left[\prod_{s=r+1}^{k-1}\left(t_{r s}^{\prime+}\right)^{p_{r s}}\right]\left|0_{g}\right\rangle_{V}, \text { for } n_{l}^{0} \in \mathbb{Z}_{2} ; n_{i j}, p_{r s} \in \mathbb{N}_{0},
$$

following the conventions (A.4) of Appendix A, Such a realization of GVM can be used if it is desirable to convert only a part of constraints from the whole system of second-class constraints (e.g., for the last example without conversion of constraints related to the $k$-th set of Lorentz indices $\mu_{1}^{k} \ldots \mu_{n_{k}}^{k}$ in the initial spin-tensor $\left.\Psi_{\left(\mu^{1}\right)_{n_{1}},\left(\mu^{2}\right)_{n_{2}}, \ldots,\left(\mu^{k}\right)_{n_{k}}}\right)$. We finally note that the case of GVM construction and the study of its properties for infinite-dimensional super-Virasoro $N=1$ algebras were examined in [75].

\section{B Oscillator Scalar Realization of Superalgerbra $o s p(1 \mid 2 k)$ in New Fock Space}

Using the general results of Burdik [72], initially elaborated for simple Lie algebras and then enlarged to special Lie superalgebras in Refs. [55], [59] (to nonlinear superalgebras for higherspin fields in $\operatorname{AdS}(d)$ spaces, in [56], 63]), and introducing a mapping between the basis of a generalized Verma module for $\operatorname{ssp}(1 \mid 2 k)$ given by the vector $\left|\vec{N}^{f}\right\rangle_{V}$ A.3 and the one a in new Fock space $\mathcal{H}^{\prime}$, we have

$$
\left|\vec{n}_{l}^{0}, \vec{n}_{i j}, \vec{p}_{r s}\right\rangle_{V} \leftrightarrow\left|\vec{n}_{l}^{0}, \vec{n}_{i j}, \vec{p}_{r s}\right\rangle=\prod_{l=1}^{k}\left(f_{l}^{+}\right)^{n_{l}^{0}} \prod_{i, j \geq i}^{k}\left(b_{i j}^{+}\right)^{n_{i j}} \prod_{r, s, s>r}^{k}\left(d_{r s}^{+}\right)^{p_{r s}}|0\rangle,
$$

with the vector $\left|\vec{n}_{l}^{0}, \vec{n}_{i j}, \vec{p}_{r s}\right\rangle$ having the same structure as $\left|\vec{N}^{f}\right\rangle_{V}$ in Eq. A.4, for $n_{l}^{0} \in \mathbb{Z}_{2}, n_{i j}, p_{r s} \in$ $\mathbb{N}_{0}$. The set of $\left|\vec{n}_{l}^{0}, \vec{n}_{i j}, \vec{p}_{r s}\right\rangle$ presents the basis vectors of a Fock space $\mathcal{H}^{\prime}$ generated by new 
fermionic, $f_{l}^{+}, f_{l}, l=1, \ldots, k$, and bosonic, $b_{i j}^{+}, d_{r s}^{+}, b_{i j}, d_{r s}, i, j, r, s=1, \ldots, k ; i \leq j ; r<s$, creation and annihilation operators with the only nonvanishing supercommutation relations

$$
\left\{f_{i}, f_{j}^{+}\right\}=\delta_{i j}, \quad\left[b_{i_{1} j_{1}}, b_{i_{2} j_{2}}^{+}\right]=\delta_{i_{1} i_{2}} \delta_{j_{1} j_{2}}, \quad\left[d_{r_{1} s_{1}}, d_{r_{2} s_{2}}^{+}\right]=\delta_{r_{1} r_{2}} \delta_{s_{1} s_{2}} .
$$

Having the correspondence (B.1), we can represent the action of the elements $o_{I}^{\prime}$ on the generalized Verma module vector $\left|\vec{N}^{f}\right\rangle_{V}$ given by Eqs. (A.5)- (A.8), (A.17)-(A.22) as polynomials in the creation and annihilation operators of the Fock space $\mathcal{H}^{\prime}$. In doing so, we have to take into account the requirement of coincidence of the numbers of the above fermionic $\left(f_{l}, f_{l}^{+}\right)$and bosonic $\left(b_{i j}^{+}, b_{i j}, d_{r s}^{+}, d_{r s}\right)$ operators with the numbers of second-class constraints, i.e., with the respective numbers of odd and even root vectors in the Cartan decomposition of $\operatorname{ssp}(1 \mid 2 k)$ (A.1).

As a result, the oscillator realization of the elements $o_{I}^{\prime}$ over the Heisenberg superalgebra $A_{k, k^{2}}$ can be presented in a unique way, first, for the Cartan elements $g_{0}^{\prime i}$ and odd $t_{i}^{\prime+}$ and even $\left(l_{i j}^{\prime+}, t_{r s}^{\prime+}\right)$ negative root vectors as follows:

$$
\begin{aligned}
g_{0}^{\prime i} & =f_{i}^{+} f_{i}+\sum_{l \leq m} b_{l m}^{+} b_{l m}\left(\delta^{i l}+\delta^{i m}\right)+\sum_{r<s} d_{r s}^{+} d_{r s}\left(\delta^{i s}-\delta^{i r}\right)+h^{i}, \\
t_{i}^{\prime+} & =f_{i}^{+}+2 b_{i i}^{+} f_{i}+4 \sum_{l=1}^{i-1} b_{l i}^{+} f_{l}, \\
l_{i j}^{\prime+} & =b_{i j}^{+}, \\
t_{r s}^{\prime+} & =d_{r s}^{+}-\sum_{n=1}^{r-1} d_{n r} d_{n s}^{+}-\sum_{n=1}^{k}\left(1+\delta_{n r}\right) b_{n s}^{+} b_{r n}-\left[4 \sum_{n=r+1}^{s-1} b_{n s}^{+} f_{n}+\left(f_{s}^{+}+2 b_{s s}^{+} f_{s}\right)\right] f_{r},
\end{aligned}
$$

second, for the odd elements $t_{i}^{\prime}$ of upper-triangular subsuperalgebra $\mathcal{E}_{k}^{+}$,

$$
\begin{aligned}
t_{i}^{\prime}= & -2 \sum_{n=1}^{i-1}\left\{\sum_{m=1}^{n-1} d_{m n}^{+} d_{m i}-\sum_{p=0}^{i-n-1} \sum_{k_{1}=n+1}^{i-1} \ldots \sum_{k_{p}=n+p}^{i-1} C^{k_{p} i}\left(d^{+}, d\right) \prod_{j=1}^{p} d_{k_{j-1} k_{j}}\right. \\
& \left.+\sum_{m=1}^{k}\left(1+\delta_{m i}\right) b_{m n}^{+} b_{m i}-\left[4 \sum_{m=n+1}^{i-1} b_{n m}^{+} f_{m}-f_{n}^{+}\right] f_{i}\right\} f_{n} \\
& +2 \sum_{n=i+1}^{k}\left\{d_{i n}^{+}-\sum_{m=1}^{i-1} d_{m n}^{+} d_{m i}-\sum_{m=1}^{k}\left(1+\delta_{m i}\right) b_{n m}^{+} b_{i m}\right\} f_{n} \\
& -2\left(\sum_{l=1}^{k}\left(1+\delta_{i l}\right) b_{i l}^{+} b_{i l}-\sum_{s>i} d_{i s}^{+} d_{i s}+\sum_{r<i} d_{r i}^{+} d_{r i}+h^{i}\right) f_{i} \\
& +\sum_{n=1}^{k}\left(1+\delta_{n i}\right)\left\{2 \sum_{m=n+1}^{k} b_{n m}^{+} f_{m}-\frac{1}{2}\left(f_{n}^{+}-2 b_{n n}^{+} f_{n}\right)\right\} b_{n i},
\end{aligned}
$$

and for even ones $l_{l m}^{\prime}$ of upper-triangular subsuperalgebra $\mathcal{E}_{k}^{+}$separately, for $l=m$ and for $l<m$

$$
\begin{aligned}
l_{l l}^{\prime}= & -\left[2 \sum_{n=l+1}^{k}\left\{d_{l n}^{+}-\sum_{n^{\prime}=1}^{l-1} d_{n^{\prime} n}^{+} d_{n^{\prime} l}-\sum_{n^{\prime}=1}^{k}\left(1+\delta_{n^{\prime} l}\right) b_{n^{\prime} n}^{+} b_{n^{\prime} l}\right\} f_{n}\right. \\
& \left.-\sum_{n=1}^{k}\left(1+\delta_{n l}\right)\left\{-2 \sum_{m=n+1}^{k} b_{n m}^{+} f_{m}+\frac{1}{2}\left[f_{n}^{+}-\left(1-\delta_{n l}\right) 2 b_{n n}^{+} f_{n}\right]\right\} b_{l n}\right] f_{l}+l_{l l}^{\prime b},
\end{aligned}
$$




$$
\begin{aligned}
l_{l m}^{\prime}= & -\left[\sum _ { n = l + 1 } ^ { m - 1 } \left\{-\sum_{n^{\prime}=1}^{n-1} d_{n^{\prime} n}^{+} d_{n^{\prime} m}+\sum_{p=0}^{m-n-1} \sum_{k_{1}=n+1}^{m-1} \ldots \sum_{k_{p}=n+p}^{m-1} C^{k_{p} m}\left(d^{+}, d\right) \prod_{j=1}^{p} d_{k_{j-1} k_{j}}\right.\right. \\
& \left.-\sum_{n^{\prime}=1}^{k}\left(1+\delta_{n^{\prime} m}\right) b_{n^{\prime} n}^{+} b_{n^{\prime} m}+\left[4 \sum_{n^{\prime}=n+1}^{m-1} b_{n^{\prime} n}^{+} f_{n^{\prime}}-f_{n}^{+}\right] f_{m}\right\} f_{n} \\
& +\sum_{n=m+1}^{k}\left\{d_{m n}^{+}-\sum_{n^{\prime}=1}^{m-1} d_{n^{\prime} n}^{+} d_{n^{\prime} m}-\sum_{n^{\prime}=1}^{k}\left(1+\delta_{n^{\prime} m}\right) b_{n^{\prime} n}^{+} b_{m n^{\prime}}\right\} f_{n} \\
& -\left(\sum_{n=1}^{k}\left(1+\delta_{n m}\right) b_{m n}^{+} b_{m n}-\sum_{s>m} d_{m s}^{+} d_{m s}+\sum_{r<m} d_{r m}^{+} d_{r m}+h^{m}\right) f_{m} \\
& \left.+\frac{1}{2} \sum_{n=1}^{k}\left(1+\delta_{n m}\right)\left\{2 \sum_{n^{\prime}=n+1}^{k} b_{n n^{\prime}}^{+} f_{n^{\prime}}-\frac{1}{2}\left[f_{n}^{+}-\left(1-\delta_{n l}\right) 2 b_{n n}^{+} f_{n}\right]\right\} b_{n m}\right] f_{l} \\
& -\left[\sum_{n=m+1}^{k}\left\{d_{l n}^{+}-\sum_{n^{\prime}=1}^{l-1} d_{n^{\prime} n}^{+} d_{n^{\prime} l}-\sum_{n^{\prime}=1}^{k}\left(1+\delta_{n^{\prime} l}\right) b_{n n^{\prime}}^{+} b_{l n^{\prime}}\right\} f_{n}\right. \\
& \left.+\frac{1}{2} \sum_{n=1}^{k}\left(1+\delta_{n l}\right)\left\{2 \sum_{n^{\prime}=n+1}^{k} b_{n n^{\prime}}^{+} f_{n^{\prime}}-\frac{1}{2}\left[f_{n}^{+}-\left(1-\delta_{n m}\right) 2 b_{n n}^{+} f_{n}\right]\right\} b_{n l}\right] f_{m}+l_{l m}^{\prime b}
\end{aligned}
$$

where the purely bosonic operators $l_{l l}^{\prime b}, l_{l m}^{\prime b}$, for $l<m$, correspond to those for the symplectic algebra $s p(2 k)$, derived from the actions of $l_{l^{\prime} l^{\prime}}^{\prime}, l_{l^{\prime} m^{\prime}}^{\prime}$ on the bosonic part of the basis vector of a generalized Verma module, $\left|\overrightarrow{0}_{l}^{0} ; \vec{N}\right\rangle_{V}$, given by Eqs. (A.20), (A.22) and first found in [31],

$$
\begin{aligned}
l_{l l}^{\prime b}= & \frac{1}{4} \sum_{n=1, n \neq l}^{k} b_{n n}^{+} b_{l n}^{2}+\frac{1}{2} \sum_{n=1}^{l-1}\left[\sum_{n^{\prime}=1}^{n-1} d_{n^{\prime} n}^{+} d_{n^{\prime} l}+\sum_{n^{\prime}=n+1}^{k}\left(1+\delta_{n^{\prime} l}\right) b_{n n^{\prime}}^{+} b_{n^{\prime} l}\right. \\
& \left.-\sum_{p=0}^{l-n-1} \sum_{k_{1}=n+1}^{l-1} \ldots \sum_{k_{p}=n+p}^{l-1} C^{k_{p} l}\left(d^{+}, d\right) \prod_{j=1}^{p} d_{k_{j-1} k_{j}}\right] b_{n l} \\
& +\left(\sum_{n=l}^{k} b_{n l}-\sum_{s>l} d_{l s}^{+} d_{l s}+\sum_{r<l} d_{r l}^{+} d_{r l}+h^{l}\right) b_{l l} \\
& -\frac{1}{2} \sum_{n=l+1}^{k}\left[d_{l n}^{+}-\sum_{n^{\prime}=1}^{l-1} d_{n^{\prime} n}^{+} d_{n^{\prime} l}-\sum_{n^{\prime}=n+1}^{k}\left(1+\delta_{n^{\prime} l}\right) b_{n^{\prime} n}^{+} b_{n^{\prime} l}\right] b_{l n}, \\
l_{l m}^{\prime b}= & -\frac{1}{4} \sum_{n=1}^{m-1}\left(1+\delta_{n l}\right)\left[-\sum_{n^{\prime}=1}^{n-1} d_{n^{\prime} n}^{+} d_{n^{\prime} m}-\sum_{n^{\prime}=n}^{k}\left(1+\delta_{n^{\prime} m}\right) b_{n^{\prime} n}^{+} b_{n^{\prime} m}\right. \\
& \left.+\sum_{p=0}^{m-n-1} \sum_{k_{1}=n+1}^{m-1} \ldots \sum_{k_{p}=n+p}^{m-1} C^{k_{p} n}\left(d^{+}, d\right) \prod_{j=1}^{m} d_{k_{j-1} k_{j}}\right] b_{n l} \\
& -\frac{1}{4} \sum_{n=m+1}^{k}\left[d_{m n}^{+}-\sum_{n^{\prime}=1}^{m-1} d_{n^{\prime} n}^{+} d_{n^{\prime} m}-\sum_{n^{\prime}=l+1}^{k}\left(1+\delta_{n^{\prime} m}\right) b_{n^{\prime} n}^{+} b_{m n^{\prime}}\right] b_{l n} \\
& +\frac{1}{4}\left(\sum_{n=m}^{k} b_{l n}^{+} b_{l n}+\sum_{n=l+1}^{k}\left(1+\delta_{n m}\right) b_{n m}^{+} b_{n m}-\sum_{s>l} d_{l s} d_{l_{s}}-\sum_{s>m} d_{m s}^{+} d_{m s}\right.
\end{aligned}
$$




$$
\begin{aligned}
& \left.+\sum_{r<l} d_{r l}^{+} d_{r l}+\sum_{r<m} d_{r m}^{+} d_{r m}+h^{l^{\prime}}+h^{m^{\prime}}\right) b_{l m} \\
& -\frac{1}{4} \sum_{n=1}^{l-1}\left[-\sum_{n^{\prime}=1}^{n-1} d_{n^{\prime} n}^{+} d_{n^{\prime} l}+\sum_{p=0}^{l-n-1} \sum_{k_{1}=n+1}^{l-1} \ldots \sum_{k_{p}=n+p}^{l-1} C^{k_{p} n}\left(d^{+}, d\right) \prod_{j=1}^{p} d_{k_{j-1} k_{j}}\right. \\
& \left.-\sum_{n^{\prime}=n+1}^{k}\left(1+\delta_{n^{\prime} l}\right) b_{n^{\prime} n}^{+} b_{n^{\prime} l}\right] b_{n m}-\frac{1}{4} \sum_{n=l+1}^{k}\left(1+\delta_{n m}\right)\left[d_{l n}^{+}-\sum_{n^{\prime}=1}^{l-1} d_{n^{\prime} n}^{+} d_{n^{\prime} l}\right] b_{m n} .
\end{aligned}
$$

Finally, for the "mixed-symmetry" operators $t_{r s}^{\prime}$ with purely fermionic input (proportional to $\left.f_{l}, f_{l}^{+}\right)$in the last row, we have

$$
\begin{aligned}
t_{r s}^{\prime}= & -\sum_{n=1}^{r-1} d_{n r}^{+} d_{n s}+\sum_{p=0}^{s-r-1} \sum_{k_{1}=r+1}^{s-1} \ldots \sum_{k_{p}=r+p}^{s-1} C^{k_{p} s}\left(d^{+}, d\right) \prod_{j=1}^{p} d_{k_{j-1} k_{j}} \\
& -\sum_{n=1}^{k}\left(1+\delta_{n s}\right) b_{n r}^{+} b_{n s}+\left[4 \sum_{n=r+1}^{s-1} b_{r n}^{+} f_{n}+\left(2 b_{r r}^{+} f_{r}-f_{r}^{+}\right)\right] f_{s}, \quad k_{0} \equiv r
\end{aligned}
$$

where the operators $C^{r s}\left(d, d^{+}\right)$are obtained from the vector $\left|C_{\vec{p}_{r^{\prime} s^{\prime}}}^{r s}\right\rangle_{V}, r<m$, determined in Eq. (A.15) by the rule (first derived in [31]),

$$
\begin{aligned}
C^{r s}\left(d^{+}, d\right) \equiv & \left(h^{r}-h^{s}-\sum_{n=s+1}^{k}\left(d_{r n}^{+} d_{r n}+d_{s n}^{+} d_{s n}\right)+\sum_{n=r+1}^{s-1} d_{n s}^{+} d_{n s}-d_{r s}^{+} d_{r s}\right) d_{r s} \\
& +\sum_{n=s+1}^{k}\left\{d_{s n}^{+}-\sum_{n^{\prime}=1}^{s-1} d_{n^{\prime} n}^{+} d_{n^{\prime} s}\right\} d_{r n} .
\end{aligned}
$$

To obtain an oscillator representation for $o_{I}^{\prime}$, we have used, for instance, following one-to-one correspondences inspired by (B.1), to find, for a given transformed vector $\left|\vec{N}^{f}\right\rangle_{V}$, a corresponding vector in $\mathcal{H}^{\prime}$,

$$
\begin{aligned}
& -n_{m} \sum_{n=l+1}^{m-1} n_{n}^{0}(-1)^{\sum_{n^{\prime}=n+1}^{m-1} n_{n^{\prime}}^{0}}\left|\vec{N}^{f}-\delta_{l n}-\delta_{l m}+\delta_{n l, i j}\right\rangle_{V} \longleftrightarrow \sum_{n=l+1}^{m-1} b_{l n}^{+} f_{n} f_{m}\left|\vec{N}^{f}\right\rangle, \\
& -n_{m}(-1)^{\sum_{n=l^{\prime}+1}^{m-1} n_{n}^{0}}\left(1+\left[\frac{n_{l^{\prime}}^{0}+1}{2}\right]\right)\left|\vec{N}^{f}+\delta_{l l^{\prime}} \bmod 2-\delta_{l m}+\delta_{l^{\prime} l^{\prime}, i j}\left[\frac{n_{l^{\prime}}^{0}+1}{2}\right]\right\rangle_{V} \longleftrightarrow \\
& \longleftrightarrow\left(2 b_{l^{\prime} l^{\prime}}^{+} f_{l^{\prime}}-f_{l^{\prime}}^{+}\right) f_{m}\left|\vec{N}^{f}\right\rangle,
\end{aligned}
$$

Let us find an explicit expression for the operator $K^{\prime}$ used in the definition of the scalar product (3.14), and given in an exact form by (3.16).

One can show by direct calculation that the following relation holds true:

$$
{ }_{V}\left\langle\vec{p}_{r s}^{\prime}, \vec{n}_{i j}^{\prime} ; \vec{n}_{l}^{\prime 0} \mid \vec{n}_{l}^{0} ; \vec{n}_{i j}, \vec{p}_{r s}\right\rangle_{V} \sim \prod_{l} \delta_{\sum_{i} n_{i}^{\prime 0}+\sum_{i}\left(1+\delta_{i l}\right) n_{i l}^{\prime}-\sum_{i>l} p_{l i}^{\prime}+\sum_{i<l} p_{i l}^{\prime}}^{\prime} .
$$

For practical calculations, with low pairs of numbers

$$
\begin{gathered}
\left(n_{1}^{0}+\sum_{i}\left(1+\delta_{i 1}\right) n_{1 i}-\sum_{i>1} p_{1 i}, n_{2}^{0}+\sum_{i}\left(1+\delta_{i 2}\right) n_{2 i}-\sum_{i>2} p_{2 i}+p_{12}, \ldots\right. \\
\left.n_{k}^{0}+\sum_{i}\left(1+\delta_{i k}\right) n_{i k}+\sum_{i<k} p_{i k}\right)
\end{gathered}
$$


with the number of "particles" associated with $, n_{l}^{0}, p_{r t}, n_{i j}$ being the numbers of Fermi and Bose "particles" associated with $f_{l}^{+}$and $d_{r t}^{+}, b_{i j}^{+}$for $i \leq j, r<t$ (where $d_{r t}^{+}$reduces the spin number $s_{r}$ by one unit and increases the spin number $s_{t}$ by one unit simultaneously), the operator $K^{\prime}$ reads, with the use of the normalization condition ${ }_{V}\langle 0 \mid 0\rangle_{V}=1$,

$$
\begin{aligned}
K^{\prime}= & |0\rangle\left\langle 0\left|+\sum_{r<s}\left(h^{r}-h^{s}\right) d_{r s}^{+}\right| 0\right\rangle\left\langle 0\left|d_{r s}+\sum_{i \leq j}\left(h^{i}\left(1+2 \delta^{i j}\right)+h^{j}\right) b_{i j}^{+}\right| 0\right\rangle\langle 0| b_{i j} \\
& -2 \sum_{i} h^{i} f_{i}^{+}|0\rangle\left\langle 0\left|f_{i}+2 \sum_{j}\left(f_{j}^{+}|0\rangle\langle 0| \sum_{i<j}\left(h^{i}-h^{j}\right) d_{i j} f_{i}\right)+2 \sum_{i<j}\left(h^{i}-h^{j}\right) f_{i}^{+} d_{i j}^{+}\right| 0\right\rangle\langle 0| f_{j} \\
& -2 \sum_{i, r<s} f_{i}^{+} d_{r s}^{+}|0\rangle\left\langle 0\left|d_{r s} f_{i}\left(h^{r}-h^{s}\right)\left(h^{i}+\delta^{i s}-\delta^{i r}\right)+2 \sum_{r<s} \sum_{i=1}^{r-1} f_{r}^{+} d_{r s}^{+}\right| 0\right\rangle\langle 0| d_{i s} f_{i}\left(h^{r}-h^{s}\right) \\
& -2 \sum_{i, s=1}^{k, i-1} \sum_{r=1}^{s-1} f_{i}^{+} d_{r s}^{+}|0\rangle\left\langle 0\left|d_{r i} f_{s}\left(h^{r}-h^{s}\right)+2 \sum_{s, i, r}^{k, s-1, i-1} f_{r}^{+} d_{r s}^{+}\right| 0\right\rangle\langle 0| d_{i s} f_{i}\left(h^{i}-h^{s}\right) \\
& -2 \sum_{s, i, r}^{k, s-1, i-1} f_{i}^{+} d_{r s}^{+}|0\rangle\langle 0| d_{r i} f_{s}\left(h^{r}-h^{i}\right)+\frac{1}{2} \sum_{l<i}\left(h^{i}-h^{l}\right)\left(b_{i i}^{+}|0\rangle\left\langle 0 \mid b_{l i} d_{l i}+b_{l i}^{+} d_{l i}^{+} 0\right\rangle\langle 0| b_{i i}\right) \\
& +\frac{1}{4} \sum_{i<j} b_{i j}^{+}|0\rangle\langle 0|\left(4 f_{j} f_{i} h^{j}+\left(1+\delta^{l i}\right) \sum_{l<j}\left(h^{j}-h^{l}\right) b_{i l} d_{l j}\right) \\
& +\frac{1}{4} \sum_{i<j}\left(\sum_{l<i} b_{l j}^{+} d_{l i}^{+}|0\rangle\left\langle 0\left|\left(h^{i}-h^{l}\right)+\sum_{l<j} b_{l j}^{+} d_{l i}^{+}\right| 0\right\rangle\langle 0|\left(1+\delta^{l i}\right)\left(h^{j}-h^{l}\right)\right) b_{i j} \\
& +\sum_{i<j} f_{i}^{+} f_{j}^{+}|0\rangle\langle 0|\left(4 f_{j} f_{i}\left(h^{j} h^{i}+h^{j}-h^{i}\right)+b_{i j} h^{j}\right)+\ldots
\end{aligned}
$$

The expression for the above operator $K^{\prime}$ can be used to construct LF for fermionic HS fields with low value of generalized spin.

Summarizing, we state that the auxiliary scalar representation of the orthosymplectic superalgebra $\operatorname{osp}(1 \mid 2 k)$ for the additional parts of constraints $o_{I}^{\prime}$ in the new Fock space $\mathcal{H}^{\prime}$ is found. In addition, note that the result contains, as a particular case, for vanishing fermionic oscillators, $f_{i}, f_{i}^{+}$, the auxiliary scalar representation of the symplectic $s p(2 k)$ algebra constructed in Ref.[31].

\section{Equivalence to Initial Irreducible Relations}

We examine here, for the most part, a massive case, and make comments on massless HS spintensors. Our purpose to show that the equations of motion (3.17), (2.3) [or, equivalently, (3.20), for $\left.\tilde{t}_{0}=t_{0}+\tilde{\gamma} m\right]$ can be achieved by using the action (5.33) after gauge-fixing and removing the auxiliary fields by using a part of the equations of motion. Let us begin with gauge-fixing.

\section{C.1 Gauge-fixing Procedure}

Our starting point is the fields $\left|\chi_{0}^{l}\right\rangle$ and the sequence of $\left|\Lambda_{0}^{(s) l}\right\rangle$, for $l=0,1, s=0, \ldots, \sum_{o=1}^{k} n_{o}+$ $k(k-1) / 2-1$, at some fixed values of $\operatorname{spin}\left(n_{1}+\frac{1}{2}, \ldots, n_{k}+\frac{1}{2}\right)$. In this section, we omit the subscripts associated with the eigenvalues of the spin operators, $\sigma_{i}$, (5.16). As a first step, we examine the lowest-level gauge transformation, for $s_{\max }=\sum_{o=1}^{k} n_{o}+k(k-1) / 2-1$,

$$
\delta\left|\Lambda_{0}^{\left(s_{\max }-1\right) 0}\right\rangle=\Delta Q\left|\Lambda_{0}^{\left(s_{\max }\right) 0}\right\rangle, \quad \delta\left|\Lambda_{0}^{\left(s_{\max }-1\right) 1}\right\rangle=\tilde{T}_{0}\left|\Lambda_{0}^{\left(s_{\max }\right)}\right\rangle,
$$


where, due to the ghost number (5.19) and spin value (5.18), we use the restrictions $\left|\Lambda^{\left(s_{\max }\right) 1}\right\rangle \equiv 0$. Indeed, the independent lowest gauge parameter $\left.\left|\Lambda^{\left(s_{\max }\right)}\right\rangle_{0}\right\rangle$ has the structure

$$
\left|\Lambda_{0}^{\left(s_{\max }\right) 0}\right\rangle=\sum_{n} \prod_{i=1}^{k}\left(\prod_{n_{p i}, n_{b i}}\left(\mathcal{P}_{i}^{+}\right)^{n_{p i}}\left(p_{i}^{+}\right)^{n_{b i}}\right) \prod_{t<u}^{k-1, k} \lambda_{t u}^{+}\left|\Lambda\left(d^{+}\right)^{\left(s_{\max }\right)(n)_{b i}(n)_{p i}(1)_{\lambda t u} 0}\right\rangle
$$

which does not contain any ghost coordinate operators from Wick pairs $C^{a}\left(\left\{C^{a}\right\} \subset\left\{C^{I}\right\}\right)$ and creation operators $a_{i}^{+}, f_{i}^{+}, b_{i}^{+}, b_{i j}^{+}$. The corresponding sums of degrees $n_{p i}, n_{b i}, n_{\lambda t u}$ satisfy the distributions given by Eqs. (5.18), (5.19. So, for the last (ghost number) relation, we have $-\left[\sum_{i}\left(n_{p i}+n_{b i}\right)+\sum_{r<u} n_{\lambda r u}\right]=-s_{\max }-1$, for $\sum_{r<u} n_{\lambda r u}=k(k-1) / 2$, whereas the ghost momentum $p_{1}^{+}$is always present in the decomposition (C.2) and the summands include the vector

$$
\left(p_{i}^{+}\right)^{\sum_{o=1} n_{o}} \prod_{t<u}^{k-1, k}\left(\lambda_{t u}^{+}\right)\left|\Lambda\left(d^{+}\right)^{\left(s_{\max }\right) n_{b 1} 0_{b 2} \ldots 0_{b k}(0)_{p i}(1)_{\lambda t u} 0}{ }_{0}^{0}\right\rangle\left(-\sum_{i \geq 2} n_{i}+k-1, n_{2}+(k-2)-1, \ldots, n_{k}+(k-k)-k+1\right)
$$

being independent, in addition, from the ghost momenta $\mathcal{P}_{1}^{+}, \ldots, \mathcal{P}_{k}^{+}, p_{2}^{+}, \ldots, p_{k}^{+}$and depends only on "mixed-symmetry" creation operators $d_{r s}^{+}$, e.g., as a multiplier $\prod_{s \geq 2}\left(d_{1 s}^{+}\right)^{n_{s}-1} \prod_{r=2, r<p} d_{r p}^{+}$.

Extracting an explicit dependence of the fields, of the gauge parameters, and of the operator $\Delta Q$ (5.25), on $q_{1}, p_{1}^{+}$, bosonic ghost coordinate and momentum,

$$
\left|\Lambda_{0}^{(s) l}\right\rangle=\left|\Lambda_{00}^{(s) l}\right\rangle+p_{1}^{+}\left|\Lambda_{01}^{(s) l}\right\rangle, \text { for } l=0,1, \Delta Q=\Delta Q_{11}+q_{1}\left(T_{11}^{0+}-2 q_{1} \mathcal{P}_{11}^{+}\right)+U_{11} p_{1}^{+},(\mathrm{C} .3)
$$

where, first, for $s=-1$ we denote, $\left|\Lambda_{0}^{(-1) l}\right\rangle \equiv\left|\chi_{0}^{l}\right\rangle$, second, the quantities $\left|\Lambda_{00}^{(s) l}\right\rangle, T_{11}^{0+}, T_{11}^{1+}, U_{11}$, $\Delta Q_{11}$ do not depend on $q_{1}, p_{1}^{+}$except for the vector $\left|\Lambda_{01}^{(s) l}\right\rangle$, we obtain the gauge transformation of $\left|\Lambda^{\left(s_{\max }-1\right) l}{ }_{00}\right\rangle$

$$
\delta\left|\Lambda^{\left(s_{\max }-1\right) 0}{ }_{01}^{0}\right\rangle=T_{11}^{0+}\left|\tilde{\Lambda}_{01}^{\left(s_{\max }\right) 0}\right\rangle-2 \mathcal{P}_{11}^{+}\left|\widehat{\Lambda}_{01}^{\left(s_{\max }\right) 0}\right\rangle .
$$

Here, we have used that $\left|\Lambda^{\left(s_{\max }\right)}{ }_{00}\right\rangle \equiv 0$, due to the decomposition (C.2), implying a ghost number restriction, and the gauge parameter $\left|\tilde{\Lambda}_{01}^{\left(s_{\max }\right) 0}{ }_{01}\right\rangle$ (but not the vector $\left|\widehat{\Lambda}_{01}^{\left(s_{\max }\right) 0}\right\rangle$ ) has the same structure as $\left|\Lambda_{0}^{\left(s_{\max } 0\right.}\right\rangle$ in (C.3) lowered by 1 degree in $p_{1}^{+}$,

$$
\begin{aligned}
& \left|\tilde{\Lambda}_{01}^{\left(s_{\max }\right) 0}\right\rangle=\sum_{n} n_{b 1}\left(\mathcal{P}_{1}^{+}\right)^{n_{p 1}}\left(p_{1}^{+}\right)^{n_{b 1}-1} \prod_{i=2}^{k}\left(\prod_{n_{p i}, n_{b i}}\left(\mathcal{P}_{i}^{+}\right)^{n_{p i}}\left(p_{i}^{+}\right)^{n_{b i}}\right) \prod_{t<u}^{k-1, k} \lambda_{t u}^{+} \\
& \times\left|\Lambda\left(d^{+}\right)^{\left(s_{\max }\right)(n)_{b i}(n)_{p i}(1)_{\lambda t u} 0}{ }_{0}\right\rangle \text {. }
\end{aligned}
$$

Since $T_{11}^{0+}=T_{1}^{+}+O(\mathcal{C})=f_{1}^{+}+\ldots$, as follows from the structure of $\Delta Q$ in Eq. (5.25), we can remove the dependence of $\left|\Lambda^{\left(s_{\max }-1\right) 0}{ }_{00}\right\rangle$ on the $f_{1}^{+}$operator, by using all the degrees of freedom of $\left|\Lambda^{\left(s_{\max }\right)}{ }_{0}\right\rangle$. Therefore, after the gauge-fixing at the lowest level of the gauge transformations, we have conditions for $\left|\Lambda^{\left(s_{\max }-1\right) 0}{ }_{00}^{0}\right\rangle$ :

$$
f_{1}\left|\Lambda_{01}^{\left(s_{\max }-1\right) 0}\right\rangle=0 \Longleftrightarrow f_{1}\left|\Lambda_{0}^{\left(s_{\max }-1\right) 0}\right\rangle=0,
$$

so that the theory becomes an $\left(s_{\max }-1\right)$-reducible gauge theory.

Let us turn to the next, $\left(s_{\max }-2\right)$, level of gauge transformation. Notice that the structure of the gauge parameter, $\left|\Lambda^{\left(s_{\max }-1\right) 1}{ }_{0}^{1}\right\rangle$, is the same as for $\left|\Lambda^{\left(s_{\max }\right) 0}\right\rangle$ in (C.2) and the gauge transformations for $\left|\Lambda_{0}^{\left(s_{\max }-2\right) l}\right\rangle, l=0,1$, have the form

$$
\begin{aligned}
\delta\left|\Lambda_{0}^{\left(s_{\max }-2\right) 0}{ }_{0}\right\rangle & =\Delta Q\left|\Lambda_{0}^{\left(s_{\max }-1\right) 0}{ }_{0}^{0}\right\rangle+\frac{1}{2}\left\{\tilde{T}_{0}, \eta_{i}^{+} \eta_{i}\right\}\left|\Lambda^{\left(s_{\max }-1\right) 1}{ }_{0}\right\rangle, \\
\delta\left|\Lambda_{0}^{\left(s_{\max }-2\right) 1}\right\rangle & =\tilde{T}_{0}\left|\Lambda^{\left(s_{\max }-1\right) 0}{ }_{0}\right\rangle+\Delta Q\left|\Lambda^{\left(s_{\max }-1\right) 1}{ }_{0}\right\rangle .
\end{aligned}
$$


Obviously, first, the vector $\left|\Lambda^{\left(s_{\max }-2\right)}{ }_{0}^{1}\right\rangle$ has the same structure as $\left|\Lambda^{\left(s_{\max }-1\right) 0}{ }_{0}\right\rangle$, and, second, the vector $\left|\Lambda^{\left(s_{\max }-1\right) 1}{ }_{0}^{1}\right\rangle$ can be used to gauge-away the dependence on the $f_{1}^{+}$oscillator in $\left|\Lambda^{\left(s_{\max }-2\right) 1}{ }_{0}^{1}\right\rangle$, as was done by $\left|\Lambda_{0}^{\left(s_{\max }\right)}\right\rangle$ for $\left|\Lambda^{\left(s_{\max }-1\right) 0}{ }_{0}\right\rangle$ in (C.6) $)$. Therefore, we have the gauge conditions

$$
f_{1}\left|\Lambda_{01}^{\left(s_{\max }-2\right) 1}\right\rangle=0 \Longleftrightarrow f_{1}\left|\Lambda^{\left(s_{\max }-2\right) 1}\right\rangle=0,
$$

Third, there arises a dependence on the $\mathcal{P}_{11}^{+}$odd ghost momentum in $\left|\Lambda^{\left(s_{\max }-1\right) 0}{ }_{0}\right\rangle$, as follows from the decomposition ( $(\mathbf{C . 2})$, where one should replace the multiplier $\left(p_{1}^{+}\right)^{2}$ by $\mathcal{P}_{11}^{+}$, thus changing the Grassmann parity of the component vectors in it, and so we extract the explicit dependence of the gauge parameters and of the operator $\Delta Q$ on the ghosts $\eta_{11}, \mathcal{P}_{11}^{+}$, in addition to decomposition (C.3),

$$
\left|\Lambda_{0}^{(s) l}\right\rangle=\left|\Lambda_{000}^{(s) l}\right\rangle+\mathcal{P}_{11}^{+}\left|\Lambda_{001}^{(s) l}\right\rangle, \text { for } l=0,1, \Delta Q=\Delta Q_{11}^{1}+\eta_{11} T_{11}^{+}+U_{11}^{1} \mathcal{P}_{11}^{+},
$$

where the quantities $\left|\Lambda_{00 p}^{(s) l}\right\rangle, p=0,1, \Delta Q_{11}^{1}, T_{11}^{+}, U_{11}^{1}$ do not depend on $\eta_{11}, \mathcal{P}_{11}^{+}$. The remaining gauge transformations in (C.7) for the parameter of $\left(s_{\max }-2\right)$ level $\left|\Lambda^{\left(s_{\max }-2\right) 0}\right\rangle$ imply

$$
\delta\left|\Lambda_{000}^{\left(s_{\max }-2\right) 0}\right\rangle=T_{11}^{+}\left|\Lambda_{001}^{\left(s_{\max }-1 p\right) 0}\right\rangle+T_{11}^{0+}\left|\tilde{\Lambda}_{000}^{\left(s_{\max }-1\right) 0}\right\rangle .
$$

Since $T_{11}^{+}=L_{11}^{+}+q_{1}^{+} p_{1}^{+}+O(\mathcal{C})=b_{11}^{+}+q_{1}^{+} p_{1}^{+} \ldots$, as follows from the structure $\Delta Q$ in Eq. (5.25), the dependence on the auxiliary oscillator $b_{11}^{+}$for $\left|\Lambda^{\left(s_{\max }-2\right) 0}{ }_{000}\right\rangle$ (however, not simultaneously for the product of ghosts $q_{1}^{+} p_{1}^{+}$) can be gauged away by the residual, due to restriction (C.6), degrees of freedom of the vector $\left|\Lambda^{\left(s_{\max }-1\right) 0}{ }_{001}\right\rangle$, whereas the dependence on $f_{1}^{+}$is removed by $\mathcal{P}_{11}^{+}$, the independent vector $\left|\tilde{\Lambda}^{\left(s_{\max }-1\right) 0}{ }_{000}\right\rangle$ having the same form as $\left|\Lambda^{\left(s_{\max }-1\right) 0}{ }_{000}\right\rangle$ with allowance for the representation (‥5). Therefore, after gauge-fixing at the $\left(s_{\max }-2\right)$-level of gauge transformations, we have the conditions for $\left|\Lambda^{\left(s_{\max }-2\right) 0}{ }_{000}\right\rangle$

$$
f_{1}\left|\Lambda_{000}^{\left(s_{\max }-2\right) 0}\right\rangle=0, b_{11} \mathcal{P}_{11}^{+}\left|\Lambda_{000}^{\left(s_{\max }-2\right) 0}\right\rangle=0, \Longleftrightarrow\left(f_{1}, b_{11}\right) \mathcal{P}_{11}^{+}\left|\Lambda^{\left(s_{\max }-2\right) 0}\right\rangle=0,
$$

so that the total set of gauge conditions for the parameters $\left|\Lambda^{\left(s_{\max }-2\right) l}{ }_{0}\right\rangle$ is listed in Eqs. (C.8), (C.11). Notice that the dependence on the operators $f_{1}^{+}, b_{11}^{+}$in $\mid \Lambda^{\left(s_{\max }-2\right) 0}{ }_{0}$ can only be in the $\mathcal{P}_{11}^{+}$-dependent summands.

Let us now turn to the next, $\left(s_{\max }-3\right)$, level of gauge transformation. Extracting explicit dependence of the gauge parameters and $\Delta Q$ on $q_{1}, p_{1}^{+}, \eta_{11}, \mathcal{P}_{11}^{+}, q_{2}, p_{2}^{+}, \eta_{12}, \mathcal{P}_{12}^{+}$, and using arguments similar to those at the previous level of gauge transformation, one can show that one can impose gauge on the vectors $\left|\Lambda^{\left(s_{\max }-3\right) l}{ }_{0}\right\rangle$ :

$$
\left(f_{1}, b_{11}\right) \mathcal{P}_{11}^{+}\left|\Lambda^{\left(s_{\max }-3\right) l}\right\rangle=0, f_{2} \mathcal{P}_{11}^{+} \mathcal{P}_{12}^{+} \Pi_{p_{1}^{+}}^{0}\left|\Lambda_{0}^{\left(s_{\max }-3\right) 0}{ }_{0}\right\rangle=0, b_{12} \mathcal{P}_{11}^{+} \mathcal{P}_{12}^{+}\left|\Lambda^{\left(s_{\max }-3\right) 0}\right\rangle=0 .
$$

In (C.12), the quantity $\Pi_{p_{1}^{+}}^{0}$ is the projector onto the $p_{1}^{+}$-independent monomials in the vector $\left|\Lambda_{0}^{\left(s_{\max }-3\right) 0}\right\rangle$, which compose the system of projectors $\left\{\Pi_{p_{1}^{+}}^{0}, \Pi_{p_{1}^{+}}^{1}\right\}$, so that $\sum_{i} \Pi_{p_{1}^{+}}^{i}=1$. To obtain these gauge conditions, one has to use some of the degrees of freedom of the gauge parameters $\left|\Lambda^{\left(s_{\max }-2\right) l}\right\rangle$, restricted by the Eqs.(C.8), (C.11).

Applying the above-described procedure, one can obtain, step-by-step, first, for $s=s_{\max }-4$,

$$
\left[\left(f_{1}, b_{11}\right) \mathcal{P}_{11}^{+},\left(f_{2} \Pi_{p_{1}^{+}}^{0}, b_{12}\right) \mathcal{P}_{11}^{+} \mathcal{P}_{12}^{+}\right]\left|\Lambda_{0}^{(s) l}\right\rangle=0,\left(f_{3} \Pi_{p_{1}^{+}}^{0} \Pi_{p_{2}^{+}}^{0}, b_{13}\right) \prod_{i}^{3} \mathcal{P}_{1 i}^{+}\left|\Lambda_{0}^{(s) 0}\right\rangle=0,
$$

(where $\Pi_{p_{2}^{+}}^{0}$ is the projector onto the $p_{2}^{+}$-independent monomials in the vector $\left|\Lambda_{0}^{(s 0}\right\rangle$ ), and, for $s=s_{\max }-5$

$$
\begin{aligned}
& \left(\left(f_{1}, b_{11}\right) \mathcal{P}_{11}^{+},\left(f_{2} \Pi_{p_{1}^{+}}^{0}, b_{12}\right) \prod_{i}^{2} \mathcal{P}_{1 i}^{+},\left(f_{3} \prod_{i}^{2} \Pi_{p_{i}^{+}}^{0}, b_{13}\right) \prod_{i}^{3} \mathcal{P}_{1 i}^{+}\right)\left|\Lambda_{0}^{(s) l}\right\rangle=0, \\
& \left(f_{4} \prod_{i}^{3} \Pi_{p_{i}^{+}}^{0}, b_{14}\right) \prod_{i}^{4} \mathcal{P}_{1 i}^{+}\left|\Lambda_{0}^{(s) 0}\right\rangle=0 .
\end{aligned}
$$


We then define the set of operators, used in (C.6)-(C.14),

$$
\begin{aligned}
{\left[\mathcal{A}^{s}\right]=} & \left(\left(f_{1}, b_{11}\right) \mathcal{P}_{11}^{+} ; \ldots,\left(f_{k} \prod_{i}^{k-1} \Pi_{p_{i}^{+}}^{0}, b_{1 k}\right) \prod_{i}^{k} \mathcal{P}_{1 i}^{+} ; b_{22} \mathcal{P}_{22}^{+} \prod_{i}^{k} \mathcal{P}_{1 i}^{+}, \ldots,\right. \\
& \left.b_{k-1 k} \prod_{i}^{k-1} \mathcal{P}_{i k}^{+} \prod_{i, j=1, i \leq j}^{k-1} \mathcal{P}_{i j}^{+}, b_{k k} \prod_{i, j=1, i \leq j}^{k} \mathcal{P}_{i j}^{+}\right), s=1, \ldots, \frac{k(k+1)}{2}, \text { and }\left[\mathcal{A}^{0}\right] \equiv f_{1} .
\end{aligned}
$$

so that, for instance, the set $\left[\mathcal{A}^{s}\right]$, for $s>k$, contains 1 -st, $k$-th and $(k+1)$-th components, equal, respectively, to $\left(\mathcal{A}^{1} ; \mathcal{A}^{k} ; \mathcal{A}^{k+1}\right)=\left(\left(f_{1}, b_{11}\right) \mathcal{P}_{11}^{+} ;\left(f_{k} \prod_{i}^{k-1} \Pi_{p_{i}^{+}}^{0}, b_{1 k}\right) \prod_{i}^{k} \mathcal{P}_{1 i}^{+} ; b_{22} \mathcal{P}_{22}^{+} \prod_{i}^{k} \mathcal{P}_{1 i}^{+}\right)$. With the help of operators (C.15), we can equivalently rewrite Eqs. (C.6)-(C.14), and all subsequent gauge conditions based on the decomposition of the gauge parameters in all fermionic ghost momenta $P_{i j}^{+}, i \leq j$, as follows (for $\mathcal{A}^{-1} \equiv 0$ ):

$$
\left[\mathcal{A}^{s}\right]\left|\Lambda_{0}^{\left(s_{\max }-s-1\right) 0}\right\rangle=0, \quad\left[\mathcal{A}_{0}^{s-1}\right]\left|\Lambda_{0}^{\left(s_{\max }-s-1\right) 1}\right\rangle=0, \quad \text { for } s=0,1, \ldots, \frac{k(k+1)}{2} .
$$

At the next step, we apply the same procedure as above, however, starting from the gauge parameters $\left|\Lambda^{\left(s_{\max }-\frac{k(k+1)}{2}-2\right) l}\right\rangle, l=0,1$, and extract from it, as well as from the operator $\Delta Q$ (5.25), the ghost coordinates and momenta $\eta_{i j}, \mathcal{P}_{i j}^{+}, i \leq j$ and $\eta_{1}, \mathcal{P}_{1}^{+}$(maintaining in the coefficients of decomposition the bosonic ghosts $q_{i}, p_{i}^{+}$as parameters). As a result, we have obtained a set of gauge conditions for the parameters $\left|\Lambda^{\left(s_{\max }-\frac{k(k+1)}{2}-2\right) l}\right\rangle$ :

$$
\left(\left[\mathcal{A}^{\frac{1}{2} k(k+1)}\right], b_{1} \mathcal{P}_{1}^{+} \prod_{i, j=1, i \leq j}^{k} \mathcal{P}_{i j}^{+}\right)\left|\Lambda^{\left(s_{\max }-\frac{k(k+1)}{2}-2\right) 0}{ }_{0}^{0}\right\rangle=0, \quad\left[\mathcal{A}^{\frac{1}{2} k(k+1)}\right]\left|\Lambda^{\left(s_{\max }-\frac{k(k+1)}{2}-2\right) 1}{ }_{0}\right\rangle=0 .
$$

As we continue the process by extraction of the ghosts $\eta_{1}, \eta_{2} \mathcal{P}_{1}^{+}, \mathcal{P}_{2}^{+}$, and so on, we find $k$ sets of gauge conditions for the parameters $\left|\Lambda^{\left(s_{\max }-\frac{k(k+3)}{2}-m\right) l}{ }_{0}\right\rangle, m=1, \ldots, k$ :

$$
\begin{aligned}
& \left(\left[\mathcal{A}^{\frac{1}{2} k(k+1)}\right], b_{1} \mathcal{P}_{1}^{+} \prod_{i, j=1, i \leq j}^{k} \mathcal{P}_{i j}^{+}, b_{2} \prod_{m}^{2} \mathcal{P}_{m}^{+} \prod_{i, j=1, i \leq j}^{k} \mathcal{P}_{i j}^{+}\right)\left|\Lambda^{\left(s_{\max }-\frac{k(k+1)}{2}-3\right) 0}{ }_{0}\right\rangle=0, \\
& \left(\left[\mathcal{A}^{\frac{1}{2} k(k+1)}\right], b_{1} \mathcal{P}_{1}^{+} \prod_{i, j=1, i \leq j}^{k} \mathcal{P}_{i j}^{+},\right)\left|\Lambda_{0}^{\left(s_{\max }-\frac{k(k+1)}{2}-3\right) 1}\right\rangle=0 \\
& \left(\left[\mathcal{A}^{\frac{1}{2} k(k+1)}\right], b_{1} \mathcal{P}_{1}^{+} \prod_{i, j=1, i \leq j}^{k} \mathcal{P}_{i j}^{+}, \ldots, b_{k} \prod_{m}^{k} \mathcal{P}_{m}^{+} \prod_{i, j=1, i \leq j}^{k} \mathcal{P}_{i j}^{+}\right)\left|\Lambda^{\left(s_{\max }-\frac{k(k+3)}{2}-1\right) 0} 0\right\rangle=0, \\
& \left(\left[\mathcal{A}^{\frac{1}{2} k(k+1)}\right], b_{1} \mathcal{P}_{1}^{+} \prod_{i, j=1, i \leq j}^{k} \mathcal{P}_{i j}^{+}, \ldots, b_{k-1} \prod_{m}^{k-1} \mathcal{P}_{m}^{+} \prod_{i, j=1, i \leq j}^{k} \mathcal{P}_{i j}^{+}\right)\left|\Lambda^{\left(s_{\max }-\frac{k(k+3)}{2}-1\right) 1}\right\rangle=0 .
\end{aligned}
$$

Finally, realizing the same algorithm, however, starting from the parameters $\left|\Lambda^{\left(s_{\max }-\frac{k(k+3)}{2}-2\right) l}{ }_{0}\right\rangle$, and extracting from it, as well as from the operator $\Delta Q$ (5.25), the ghost coordinates and momenta $\eta_{m}, \mathcal{P}_{m}^{+}, \eta_{i j}, \mathcal{P}_{i j}^{+}, i \leq j$ and $\vartheta_{p s}, \lambda_{p s}^{+}$, for $p<s$, with a parametric dependence on $q_{i}, p_{i}^{+}$, we obtain 
$\frac{1}{2} k(k-3)$ sets of gauge conditions for the parameters $\left|\Lambda^{\left(s_{\max }-\frac{k(k+3)}{2}-1-m\right) l}\right\rangle$, for $m=1, \ldots, \frac{1}{2} k(k-1)$

$$
\begin{aligned}
& \left(\left[\mathcal{B}^{\frac{1}{2} k(k+3)}\right], d_{12} \lambda_{12}^{+} \prod_{i, j=1, i \leq j}^{k} \mathcal{P}_{i j}^{+}, b_{2} \prod_{m}^{2} \mathcal{P}_{m}^{+} \prod_{i, j=1, i \leq j}^{k} \mathcal{P}_{i j}^{+}\right)\left|\Lambda^{\left(s_{\max }-\frac{k(k+3)}{2}-2\right) 0}\right\rangle=0, \\
& {\left[\mathcal{B}^{\frac{1}{2} k(k+3)}\right],\left|\Lambda^{\left(s_{\max }-\frac{k(k+3)}{2}-2\right) 1}\right\rangle=0 ;} \\
& \left(\left[\mathcal{B}^{\frac{1}{2} k(k+3)}\right], d_{12} \lambda_{12}^{+} \prod_{i, j=1, i \leq j}^{k} \mathcal{P}_{i j}^{+}, \ldots, d_{k-1 k} \prod_{p, s=1, p<s}^{k} \lambda_{p s}^{+} \prod_{i, j=1, i \leq j}^{k} \mathcal{P}_{i j}^{+}\right)\left|\Lambda^{\left(s_{\max }-k(k+1)-1\right) 0}\right\rangle=0 \\
& \left(\left[\mathcal{B}^{\frac{1}{2} k(k+3)}\right], d_{12} \lambda_{12}^{+} \prod_{i, j=1, i \leq j}^{k} \mathcal{P}_{i j}^{+}, \ldots, d_{k-2 k} \prod_{r}^{k-2} \lambda_{r k}^{+} \prod_{p, s=1, p<s}^{k-1} \lambda_{p s}^{+} \prod_{i, j=1, i \leq j}^{k} \mathcal{P}_{i j}^{+}\right) \\
& \left|\Lambda^{\left(s_{\max }-k(k+1)-1\right) 1}{ }_{0}\right\rangle=0 .
\end{aligned}
$$

The set of the operators $\left[\mathcal{B}^{r}\right]$ is determined in Eqs. (C.20), (C.21) from Eqs. (C.19) as

$$
\left[\mathcal{B}^{\frac{1}{2} k(k+3)}\right]=\left(\left[\mathcal{A}^{\frac{1}{2} k(k+1)}\right], b_{1} \mathcal{P}_{1}^{+} \prod_{i, j=1, i \leq j}^{k} \mathcal{P}_{i j}^{+}, \ldots, b_{k} \prod_{m}^{k} \mathcal{P}_{m}^{+} \prod_{i, j=1, i \leq j}^{k} \mathcal{P}_{i j}^{+}\right)
$$

Because of absence of unused odd ghosts in the rest set of gauge parameters $\left|\Lambda^{\left(s_{\max }-k(k+1)-1-m\right) l}\right\rangle$, for $m=1, \ldots\left(s_{\max }-k(k+1)-1\right)$, we derive, as a result of the above procedure, the same gauge conditions as in (C.21):

$$
\begin{aligned}
& {\left[\mathcal{C}^{k(k+1)}\right]\left|\Lambda^{\left(s_{\max }-k(k+1)-1-m\right) l}\right\rangle=0, \text { for } l=0,1} \\
& {\left[\mathcal{C}^{k(k+1)}\right]=\left(\left[\mathcal{B}^{\frac{1}{2} k(k+3)}\right], d_{12} \lambda_{12}^{+} \prod_{i, j=1, i \leq j}^{k} \mathcal{P}_{i j}^{+}, \ldots, d_{k-1 k} \prod_{p, s=1, p<s}^{k} \lambda_{p s}^{+} \prod_{i, j=1, i \leq j}^{k} \mathcal{P}_{i j}^{+}\right) .}
\end{aligned}
$$

Finally, the gauge conditions for the fields $\left|\chi_{0}^{l}\right\rangle$ have the form

$$
\left[\mathcal{C}^{k(k+1)}\right]\left|\chi_{0}^{l}\right\rangle=0
$$

in terms of the operator-valued set $\left[\mathcal{C}^{k(k+1)}\right]$ introduced in Eqs. (C.24).

Let us now turn to removing the auxiliary fields, using the equations of motion.

\section{C.2 Auxiliary Fields Removal by Solution of Equations of Motion}

In the beginning, we decompose the fields $\chi^{l} \equiv\left|\chi_{0}^{l}\right\rangle$ as follows:

$$
\begin{gathered}
\left|\chi^{l}\right\rangle=\left|\chi_{0}^{l}\right\rangle+\mathcal{P}_{11}^{+}\left|\chi_{1}^{l}\right\rangle, \quad\left|\chi_{(0)_{\frac{1}{2} k(k+1)}^{l}}^{l}\right\rangle=\left|\chi_{(0)_{\frac{1}{2} k(k+1)} 0}^{l}\right\rangle+\mathcal{P}_{1}^{+}\left|\chi_{(0)_{\frac{1}{2} k(k+1)}}^{l}\right\rangle, \\
\left|\chi_{0}^{l}\right\rangle=\left|\chi_{00}^{l}\right\rangle+\mathcal{P}_{12}^{+}\left|\chi_{01}^{l}\right\rangle,\left|\chi_{(0)_{\frac{1}{2} k(k+1)} 0}^{l}\right\rangle=\left|\chi_{(0)_{\frac{1}{2} k(k+1)+2}}^{l}\right\rangle+\mathcal{P}_{2}^{+}\left|\chi_{(0)_{\frac{1}{2} k(k+1)} 01}^{l}\right\rangle, \\
\ldots, \\
\ldots, \\
\left|\chi_{(0)_{k}}^{l}\right\rangle=\left|\chi_{(0)_{k} 0}^{l}\right\rangle+\mathcal{P}_{1 k}^{+}\left|\chi_{(0)_{k} 1}^{l}\right\rangle, \quad\left|S_{(0)_{\frac{1}{2} k(k+3)}}^{0}\right\rangle=\left|\chi_{(0)_{\frac{1}{2} k(k+3)} 0}^{l}\right\rangle+\mathcal{P}_{k}^{+}\left|\chi_{(0)_{\frac{1}{2} k(k+3)} 1}^{l}\right\rangle \\
\left|\chi_{(0)_{k+1}}^{l}\right\rangle=\left|\chi_{(0)_{k+2}}^{l}\right\rangle+\mathcal{P}_{22}^{+}\left|\chi_{(0)_{k+1} 1}^{l}\right\rangle, \quad\left|\chi_{(0)_{\frac{1}{2} k(k+3)} 0}^{l}\right\rangle=\left|\chi_{(0)_{\frac{1}{2} k(k+3)} 00}^{l}\right\rangle+\lambda_{12}^{+}\left|\chi_{(0)_{\frac{1}{2} k(k+3)} 01}^{l}\right\rangle \\
\ldots, \quad \ldots, \\
\left|\chi_{(0)_{\frac{1}{2} k(k+1)-1}}^{l}\right\rangle=\left|\chi_{(0)_{\frac{1}{2} k(k+1)}}^{l}\right\rangle+\mathcal{P}_{k k}^{+}\left|\chi_{(0)_{\frac{1}{2} k(k+1)-1} 1}^{l}\right\rangle,\left|\chi_{(0)_{k(k+1)-1}}^{l}\right\rangle=\left|\chi_{(0)_{k(k+1)}}^{l}\right\rangle+\lambda_{k-1 k}^{+}\left|\chi_{(0)_{k(k+1)-1} 1}^{l}\right\rangle
\end{gathered}
$$


It should be noted that, due to $g h\left(\left|\chi^{l}\right\rangle\right)=-l$ and the spin value, first, the term independent on the ghost momenta is absent from the vector (Dirac-spinor) $\left|\chi^{1}\right\rangle$, i.e., $\left|\chi_{0}^{1}\right\rangle=0$, whereas the vector $\left|\chi_{(0)_{k(k+1)}^{0}}^{0}\right\rangle$ contains the physical vector $|\Psi\rangle(2.6)$ for the vanishing ghost momenta $p_{i}^{+}$, for $i=1, \ldots, k$, in view of the representation following from Eq. (5.8), and besides

$$
\left|\chi_{(0)_{k(k+1)}}^{0}\right\rangle_{(n)_{k}}=\sum_{n} \prod_{i=1}^{k}\left(q_{i}^{+}\right)^{n_{a i}}\left(p_{i}^{+}\right)^{n_{b i}}\left(\eta_{i}^{+}\right)^{n_{f i}}\left|\Psi\left(a_{i}^{+}\right)_{0}^{(n)_{a i}(n)_{b i}(n)_{f i}}\right\rangle_{\left(n-n_{a}-n_{b}-n_{f}\right)_{k}},
$$

under the requirement that all the summands should obey the relations $\left(n-n_{a}-n_{b}-n_{f}\right)_{k} \geq$ $(0)_{k}$, due to the absence of the auxiliary even oscillators $f_{i}^{+}, b_{i}^{+}, b_{i j}^{+}, d_{r s}^{+}$[as a consequence of the gauge conditions (C.25)] in the vectors $|\Psi \ldots\rangle$ in Eq. (C.31). Indeed, for $\left(n_{b}\right)_{k}=(0)_{k}$, we have

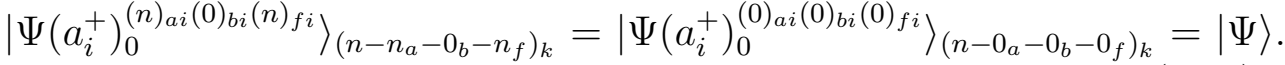

After that, by analogy with the fields, we extract from $\Delta Q$ (5.25), first, the dependence on $\eta_{11}, \mathcal{P}_{11}^{+}, \eta_{12}, \mathcal{P}_{12}^{+}, \ldots, \eta_{1 k}, \mathcal{P}_{1 k}^{+}$, next, the dependence on $\eta_{l}, \mathcal{P}_{l}^{+}, l=1, \ldots, k$, and on $\vartheta_{p s}, \lambda_{p s}^{+}$, $p<s$, respectively.

Substituting these $k(k+1)$ decompositions into the equations of motion

$$
\Delta Q\left|\chi_{0}^{0}\right\rangle+\frac{1}{2}\left\{\tilde{T}_{0}, \eta_{i}^{+} \eta_{i}\right\}\left|\chi_{0}^{1}\right\rangle=0, \quad \tilde{T}_{0}\left|\chi_{0}^{0}\right\rangle+\Delta Q\left|\chi_{0}^{1}\right\rangle=0
$$

and using the gauge conditions (C.25), one can show that, first, $\left|\chi_{(0)_{k(k+1)}^{1}}^{1}\right\rangle=0$ in (‥32), second, $\left|\chi_{(0)_{k(k+1)}^{0}}^{0}\right\rangle=0$ for the $p_{i}^{+}$-dependent vector in ( $(\overline{\mathrm{C} .32})$, so that only the original $|\Psi\rangle$ vector survives

in $\left|\chi_{(0)_{k(k+1)}}^{0}\right\rangle$, and then we obtain that $\left|\chi_{(0)_{k(k+1)-1}}^{1}\right\rangle=0$ from the second equation and $\left|\chi_{(0)_{k(k+1) 1}}^{0}\right\rangle=$ 0 from the first one in (C.32), and so on, until $\left|\chi_{0}^{1}\right\rangle=0$ and $\left|\chi_{1}^{0}\right\rangle=0$, which implies

$$
\begin{aligned}
& \Delta Q\left|\chi_{0}^{0}\right\rangle=0, \quad \tilde{t}_{0}\left|\chi^{0}\right\rangle=0, \quad\left|\chi^{1}\right\rangle=0, \\
& \left|\chi_{(0)_{k(k+1)-1} 1}^{0}\right\rangle=\left|\chi_{(0)_{k(k+1)-2} 1}^{0}\right\rangle=\ldots=\left|\chi_{01}^{0}\right\rangle=\left|\chi_{1}^{0}\right\rangle=0 .
\end{aligned}
$$

Eqs. (C.33) and (C.34) imply that all the auxiliary fields vanish and, as a result, we have $\left|\chi^{0}\right\rangle_{(n)_{k}}=|\Psi\rangle$, and the equations of motion (3.17), (2.3), (2.4) hold true. Thus, we have proved that the space of BRST cohomologies of the operator $Q$ (5.3) with a vanishing ghost number is determined only by the constraints (2.13), (2.8), corresponding to an irreducible Poincare-group representation with a given spin.

It should be noted that in the massless case the above proof of one-to-one correspondence of the Lagrangian equations of motion (5.31), (5.32) to Eqs. (3.17), (2.3), (2.4) becomes slightly corrected, because the $k$ gauge fixing conditions (C.17)-C.19) doe not hold true, and in the remaining Eqs.(C.20)-(C.25) there are no operators $b_{i} \prod_{s=1}^{i} \mathcal{P}_{s}^{+}, i=1, \ldots, k$. However, we can straightforwardly prove the validity of the same conclusion as for massive fermionic HS fields in the Lagrangian formulation for massless fermionic HS fields.

\section{Decomposition of Fields and Gauge Fock Space Vectors for Spin $\mathbf{s}=\left(\frac{5}{2}, \frac{3}{2}\right)$ spin-tensor}

We examine here only the structure of corresponding Fock space $\mathcal{H}_{\text {tot }}$ vectors $\left|\chi_{0}^{l}\right\rangle_{(2,1)},\left|\Lambda_{0}^{(0) l}\right\rangle_{(2,1)}$, $\left|\Lambda_{0}^{(2) l}\right\rangle_{(2,1)},\left|\Lambda_{0}^{(3) l}\right\rangle_{(2,1)}$, for $l=0,1,\left|\Lambda_{0}^{(3) 0}\right\rangle_{(2,1)}$ in (5.34) -(5.37) for the example of Subsection 6.3 to be used in a Lagrangian formulation for a massless (and then in Subsection 6.4 for massive) spin-tensor $\Psi_{\mu \nu, \rho}$ in a $d$-dimensional flat space-time, which is characterized by the Poincare-group 
irreducible coniditions (2.2) $-(2.4)$ and a hook-like Young tableux \begin{tabular}{|l|l}
\hline$\mu$ & $\nu$ \\
\hline$\rho$
\end{tabular} . It should be noted that the maximal stage of reducibility, $L_{k}=\sum_{o=1}^{k} n_{o}+\frac{1}{2} k(k-1)-1$, for a spin-tensor with $k$ group of symmetric indices is reached for any Young tableaux, in contrast to the case of bosonic HS fields [31. Thus, in the case of $k=2$ rows, the value $L_{2}=n_{1}+n_{2}$ is the stage of reducibility for any spin-tensor $\Psi_{(\mu)_{n_{1}},(\nu)_{n_{2}}}$, and for $\Psi_{\mu \nu, \rho}$ we have $L_{2}=3$.

Therefore, $\left|\Lambda_{0}^{(3)}\right\rangle_{(2,1)} \equiv 0$ identically, whereas the lowest fermionic independent gauge parameter $\left|\Lambda_{0}^{(3) 0}\right\rangle_{(2,1)}$ in the general expression (5.8), subject to the spin (6.5) and ghost number (6.7) conditions for $i=1,2$ and $s=3$, (for the minimal ghost number $g h_{\min }=-4$ ), has a representation with 2 summands:

$$
\left|\Lambda_{0}^{(3) 0}\right\rangle_{(2,1)}=\left(p_{1}^{+}\right)^{2} \lambda_{12}^{+}\left(p_{1}^{+} \tilde{\gamma}\left|\psi_{1}^{(3) 0}\right\rangle_{(0)}+\mathcal{P}_{1}^{+}\left|\psi_{2}^{(3) 0}\right\rangle_{(0)}\right), \quad\left|\psi_{m}^{(3) 0}\right\rangle_{(0)}=|0\rangle \psi_{m}^{(3) 0}(x),
$$

for the Dirac-spinors $\psi_{m}^{(3) 0}, m=1,2$, and where we have used the notation $(0) \equiv(0,0)$, for $\left|\psi_{m}^{(3) 0}\right\rangle_{(0)} \equiv\left|\psi_{m}^{(3)}{ }_{m}^{0}\right\rangle_{(0,0)}$.

For the bosonic reducible gauge parameters $\left|\Lambda_{0}^{(2) l}\right\rangle_{(2,1)}$ of the second level for $s=2$ in Eq.(6.7), we have a decomposition in odd (for $l=0$ ) and even (for $l=1$ ) powers of the ghosts, starting from the third (for $l=0$ ) and fourth (for $l=1$ ) powers of the ghost momenta, and consisting of $(10+2)$ summands, for $l=0,1$, respectively,

$$
\begin{aligned}
\left|\Lambda_{0}^{(2) 0}\right\rangle_{(2,1)}= & p_{1}^{+}\left(p _ { 1 } ^ { + } \left\{p_{1}^{+} \tilde{\gamma}\left|\psi_{1}^{(2) 0}\right\rangle_{(-1,1)}+p_{2}^{+} \tilde{\gamma}\left|\psi_{2}^{(2) 0}\right\rangle_{(0)}+\mathcal{P}_{1}^{+}\left|\psi_{3}^{(2) 0}\right\rangle_{(-1,1)}+\mathcal{P}_{2}^{+}\left|\psi_{4}^{(2) 0}\right\rangle_{(0)}\right.\right. \\
& \left.+\lambda_{12}^{+}\left|\psi_{5}^{(2) 0}\right\rangle_{(1,0)}\right\}+\mathcal{P}_{1}^{+}\left\{p_{2}^{+}\left|\psi_{6}^{(2) 0}\right\rangle_{(0)}+\mathcal{P}_{2}^{+} \tilde{\gamma}\left|\psi_{7}^{(2) 0}\right\rangle_{(0)}+\lambda_{12}^{+} \tilde{\gamma}\left|\psi_{8}^{(2) 0}\right\rangle_{(1,0)}\right\} \\
& \left.+\mathcal{P}_{11}^{+} \lambda_{12}^{+} \tilde{\gamma}\left|\psi_{9}^{(2) 0}\right\rangle_{(0)}\right)+\mathcal{P}_{1}^{+} \mathcal{P}_{11}^{+} \lambda_{12}^{+}\left|\psi_{10}^{(2) 0}\right\rangle_{(0)}, \\
\left|\Lambda_{0}^{(2) 1}\right\rangle_{(2,1)}= & \left(p_{1}^{+}\right)^{2} \lambda_{12}^{+}\left(p_{1}^{+}\left|\psi_{1}^{(2) 1}\right\rangle_{(0)}+\mathcal{P}_{1}^{+} \tilde{\gamma}\left|\psi_{2}^{(2) 1}\right\rangle_{(0)}\right),
\end{aligned}
$$

where the decomposition of ghost-independent vectors in powers of the initial and auxiliary creation operators in $\mathcal{H} \otimes \mathcal{H}^{\prime}$ is written as

$$
\begin{array}{ll}
\left|\psi_{m}^{(2) 1}\right\rangle_{(0)}=|0\rangle \psi_{m}^{(2) 1}(x), & \left|\psi_{n}^{(2) 0}\right\rangle_{(0)}=|0\rangle \psi_{n}^{(2) 0}(x), \\
\left|\psi_{p}^{(2) 0}\right\rangle_{(1,0)}=a_{1}^{+\mu}|0\rangle \psi_{p \mid \mu}^{(2) 0}(x)+f_{1}^{+} \tilde{\gamma}|0\rangle \psi_{p}^{(2) 0}(x), & \left|\psi_{{ }_{o}}^{(2) 0}\right\rangle_{(-1,1)}=d_{12}^{+}|0\rangle \psi_{o}^{(2) 0}(x),
\end{array}
$$

for $m=1,2, n=2,4,6,7,9,10, o=1,3$ and $p=5,8$.

For reducible fermionic gauge parameters of the first level $\left|\Lambda_{0}^{(1) l}\right\rangle_{(2,1)}$ for $s=1$ in Eq.(6.7), and for the same spin value $(2,1)$ in the Eqs.(6.5), we obtain, using the general expression (5.8), a decomposition in even (for $l=0$ ) and odd (for $l=1$ ) powers of ghosts, consisting of $(21+10)$ summands, for $l=0,1$, and starting from the second order in $\mathcal{P}_{I}$ (from the third order in ghost momenta $\mathcal{P}_{I}$ for $l=1$ ),

$$
\begin{aligned}
\left|\Lambda_{0}^{(1) 0}\right\rangle_{(2,1)}= & p_{1}^{+}\left(p _ { 1 } ^ { + } \left\{\left|\psi_{1}^{(1) 0}\right\rangle_{(0,1)}+p_{1}^{+} \vartheta_{12}^{+} \tilde{\gamma}\left|\psi_{2}^{(1) 0}\right\rangle_{(0)}+\mathcal{P}_{1}^{+} \vartheta_{12}^{+}\left|\psi_{3}^{(1) 0}\right\rangle_{(0)}+q_{1}^{+} \lambda_{12}^{+} \tilde{\gamma}\left|\psi_{4}^{(1) 0}\right\rangle_{(0)}\right.\right. \\
& \left.+\eta_{1}^{+} \lambda_{12}^{+}\left|\psi_{5}^{(1) 0}\right\rangle_{(0)}\right\}+\mathcal{P}_{1}^{+}\left\{\tilde{\gamma}\left|\psi_{6}^{(1) 0}\right\rangle_{(0,1)}+q_{1}^{+} \lambda_{12}^{+}\left|\psi_{7}^{(1) 0}\right\rangle_{(0)}+\eta_{1}^{+} \lambda_{12}^{+} \tilde{\gamma}\left|\psi_{8}^{(1) 0}\right\rangle_{(0)}\right\} \\
& +p_{2}^{+}\left|\psi_{9}^{(1) 0}\right\rangle_{(1,0)}+\mathcal{P}_{2}^{+} \tilde{\gamma}\left|\psi_{10}^{(1) 0}\right\rangle_{(1,0)}+\mathcal{P}_{11}^{+} \tilde{\gamma}\left|\psi_{11}^{(1) 0}\right\rangle_{(-1,1)}+\mathcal{P}_{12}^{+} \tilde{\gamma}\left|\psi_{12}^{(1) 0}\right\rangle_{(0)}
\end{aligned}
$$




$$
\begin{aligned}
& \left.+\lambda_{12}^{+} \tilde{\gamma}\left|\psi_{13}^{(1) 0}\right\rangle_{(2,0)}\right)+\mathcal{P}_{1}^{+}\left(p_{2}^{+} \tilde{\gamma}\left|\psi_{14}^{(1) 0}\right\rangle_{(1,0)}+\mathcal{P}_{2}^{+}\left|\psi_{15}^{(1) 0}\right\rangle_{(1,0)}+\mathcal{P}_{11}^{+}\left|\psi_{16}^{(1) 0}\right\rangle_{(-1,1)}\right. \\
& \left.+\mathcal{P}_{12}^{+}\left|\psi_{17}^{(1) 0}\right\rangle_{(0)}+\lambda_{12}^{+}\left|\psi_{18}^{(1) 0}\right\rangle_{(2,0)}\right)+p_{2}^{+} \mathcal{P}_{11}^{+} \tilde{\gamma}\left|\psi_{19}^{(1) 0}\right\rangle_{(0)}+\mathcal{P}_{2}^{+} \mathcal{P}_{11}^{+}\left|\psi_{20}^{(1) 0}\right\rangle_{(0)} \\
& +\mathcal{P}_{11}^{+} \lambda_{12}^{+}\left|\psi_{21}^{(1) 0}\right\rangle_{(1,0)}, \\
\left|\Lambda_{0}^{(1) 1}\right\rangle_{(2,1)}= & p_{1}^{+}\left(p _ { 1 } ^ { + } \left\{p_{1}^{+}\left|\psi_{1}^{(1)}{ }_{1}^{1}\right\rangle_{(-1,1)}+p_{2}^{+}\left|\psi_{2}^{(1)}{ }_{2}^{1}\right\rangle_{(0)}+\mathcal{P}_{1}^{+} \tilde{\gamma}\left|\psi_{3}^{(1) 1}\right\rangle_{(-1,1)}+\mathcal{P}_{2}^{+} \tilde{\gamma}\left|\psi_{4}^{(1) 1}\right\rangle_{(0)}\right.\right. \\
& \left.+\lambda_{12}^{+} \tilde{\gamma}\left|\psi_{5}^{(1) 1}\right\rangle_{(1,0)}\right\}+\mathcal{P}_{1}^{+}\left\{p_{2}^{+} \tilde{\gamma}\left|\psi_{6}^{(1)}\right\rangle_{(0)}+\mathcal{P}_{2}^{+}\left|\psi_{7}^{(1)}{ }_{7}^{1}\right\rangle_{(0)}+\lambda_{12}^{+}\left|\psi_{8}^{(1)}{ }_{8}\right\rangle_{(1,0)}\right\} \\
& \left.+\mathcal{P}_{11}^{+} \lambda_{12}^{+}\left|\psi_{9}^{(1) 1}\right\rangle_{(0)}\right)+\mathcal{P}_{1}^{+} \mathcal{P}_{11}^{+} \lambda_{12}^{+} \tilde{\gamma}\left|\psi_{10}^{(1) 1}\right\rangle_{(0)}
\end{aligned}
$$

where the ghost-independent vectors $\left|\psi_{n}^{(1) 1}\right\rangle_{(0)},\left|\psi_{p}^{(1)}\right\rangle_{(1,0)}$ for $n=2,4,6,7,9,10, o=1,3$ and $p=5,8$ in $\mathcal{H} \otimes \mathcal{H}^{\prime}$ have the same decomposition and the properties as those in Eqs. (D.4), (D.5), whereas the decomposition of ghost-independent vectors in Eq. (D.6), different from those in Eqs. (D.4), (D.5), reads

$$
\begin{aligned}
& \left|\psi_{n}^{(1) 0}\right\rangle_{(0,1)}=a_{1}^{+\mu} d_{12}^{+}|0\rangle \psi_{n}^{\prime(1) 0}{ }_{n \mid \mu}+a_{2}^{+\mu}|0\rangle \psi_{n \mid \mu}^{(1) 0}+f_{1}^{+} d_{12}^{+} \tilde{\gamma}|0\rangle \psi_{n}^{\prime(1) 0}+f_{2}^{+} \tilde{\gamma}|0\rangle \psi_{n}^{(1) 0}, \\
& \left|\psi_{r}^{(1) 0}\right\rangle_{(2,0)}=a_{1}^{+\mu}\left(a_{1}^{+\nu}|0\rangle \psi_{r \mid \mu \nu}^{(1) 0}+f_{1}^{+} \tilde{\gamma}|0\rangle \psi_{r \mid \mu}^{(1) 0}\right)+b_{11}^{+}|0\rangle \psi_{r}^{(1) 0},
\end{aligned}
$$

for $n=1,6, r=13,18$.

Then, for the reducible bosonic gauge parameter of the zeroth level (proper gauge parameters) $\left|\Lambda_{0}^{(0) l}\right\rangle_{(2,1)} \equiv\left|\Lambda_{0}^{l}\right\rangle_{(2,1)}$ for $s=0$ in the Eq.(6.7) and for the same spin value $(2,1)$ in Eqs.(6.5), we obtain, from the general expression (5.8), a decomposition in odd (for $l=0$ ) and even( for $l=1$ ) powers of ghosts, consisting of $(35+21)$ summands, respectively, for $l=0,1$, and starting from the first order in the ghost momenta $\mathcal{P}_{I}$ (from the second order in ghost momenta $\mathcal{P}_{I}$ for $l=1$ ),

$$
\begin{aligned}
\left|\Lambda_{0}^{0}\right\rangle_{(2,1)}= & p_{1}^{+}\left(\tilde{\gamma}\left|\psi_{1}^{0}\right\rangle_{(1,1)}+p_{1}^{+}\left\{q_{1}^{+} \tilde{\gamma}\left|\psi_{2}^{0}\right\rangle_{(-1,1)}+\eta_{1}^{+}\left|\psi_{3}^{0}\right\rangle_{(-1,1)}+q_{2}^{+} \tilde{\gamma}\left|\psi_{4}^{0}\right\rangle_{(0)}+\eta_{2}^{+}\left|\psi_{5}^{0}\right\rangle_{(0)}\right.\right. \\
& \left.+\vartheta_{12}^{+}\left|\psi_{6}^{0}\right\rangle_{(1,0)}\right\}+\mathcal{P}_{1}^{+}\left\{q_{1}^{+}\left|\psi_{7}^{0}\right\rangle_{(-1,1)}+\eta_{1}^{+} \tilde{\gamma}\left|\psi_{8}^{0}\right\rangle_{(-1,1)}+q_{2}^{+}\left|\psi_{9}^{0}\right\rangle_{(0)}+\eta_{2}^{+} \tilde{\gamma}\left|\psi_{10}^{0}\right\rangle_{(0)}\right. \\
& \left.+\vartheta_{12}^{+} \tilde{\gamma}\left|\psi_{11}^{0}\right\rangle_{(1,0)}\right\}+p_{2}^{+}\left\{q_{1}^{+} \tilde{\gamma}\left|\psi_{12}^{0}\right\rangle_{(0)}+\eta_{1}^{+}\left|\psi_{13}^{0}\right\rangle_{(0)}\right\}+\mathcal{P}_{2}^{+}\left\{q_{1}^{+}\left|\psi_{14}^{0}\right\rangle_{(0)}+\eta_{1}^{+} \tilde{\gamma}\left|\psi_{15}^{0}\right\rangle_{(0)}\right\} \\
& \left.+\mathcal{P}_{11}^{+} \vartheta_{12}^{+} \tilde{\gamma}\left|\psi_{16}^{0}\right\rangle_{(0)}+\lambda_{12}^{+}\left\{q_{1}^{+}\left|\psi_{17}^{0}\right\rangle_{(1,0)}+\eta_{1}^{+} \tilde{\gamma}\left|\psi_{18}^{0}\right\rangle_{(1,0)}+\eta_{11}^{+} \tilde{\gamma}\left|\psi_{19}^{0}\right\rangle_{(0)}\right\}\right) \\
& +p_{2}^{+}\left(\tilde{\gamma}\left|\psi_{20}^{0}\right\rangle_{(2,0)}+\mathcal{P}_{1}^{+}\left\{q_{1}^{+}\left|\psi_{21}^{0}\right\rangle_{(0)}+\eta_{1}^{+} \tilde{\gamma}\left|\psi_{22}^{0}\right\rangle_{(0)}\right\}\right)+\mathcal{P}_{2}^{+}\left(\left|\psi_{23}^{0}\right\rangle_{(2,0)}\right. \\
& \left.+\mathcal{P}_{1}^{+}\left\{q_{1}^{+} \tilde{\gamma}\left|\psi_{24}^{0}\right\rangle_{(0)}+\eta_{1}^{+}\left|\psi_{25}^{0}\right\rangle_{(0)}\right\}\right)+\mathcal{P}_{1}^{+}\left(\left|\psi_{26}^{0}\right\rangle_{(1,1)}+\mathcal{P}_{11}^{+} \vartheta_{12}^{+} \tilde{\gamma}\left|\psi_{27}^{0}\right\rangle_{(0)}\right. \\
& \left.+\lambda_{12}^{+}\left\{q_{1}^{+} \tilde{\gamma}\left|\psi_{28}^{0}\right\rangle_{(1,0)}+\eta_{1}^{+}\left|\psi_{29}^{0}\right\rangle_{(1,0)}+\eta_{11}^{+}\left|\psi_{30}^{0}\right\rangle_{(0)}\right\}\right)+\mathcal{P}_{11}^{+}\left(\left|\psi_{31}^{0}\right\rangle_{(0,1)}\right. \\
& \left.+\lambda_{12}^{+}\left\{q_{1}^{+} \tilde{\gamma}\left|\psi_{32}^{0}\right\rangle_{(0)}+\eta_{1}^{+}\left|\psi_{33}^{0}\right\rangle_{(0)}\right\}\right)+\mathcal{P}_{12}^{+}\left|\psi_{34}^{0}\right\rangle_{(1,0)}+\lambda_{12}^{+}\left|\psi_{35}^{0}\right\rangle_{(3,0)},
\end{aligned}
$$




$$
\begin{aligned}
\left|\Lambda_{0}^{1}\right\rangle_{(2,1)}= & p_{1}^{+}\left(p _ { 1 } ^ { + } \left\{\tilde{\gamma}\left|\psi_{1}^{1}\right\rangle_{(0,1)}+p_{1}^{+} \vartheta_{12}^{+}\left|\psi_{2}^{1}\right\rangle_{(0)}+\mathcal{P}_{1}^{+} \vartheta_{12}^{+} \tilde{\gamma}\left|\psi_{3}^{1}\right\rangle_{(0)}+q_{1}^{+} \lambda_{12}^{+}\left|\psi_{4}^{1}\right\rangle_{(0)}\right.\right. \\
& \left.+\eta_{1}^{+} \lambda_{12}^{+} \tilde{\gamma}\left|\psi_{5}^{1}\right\rangle_{(0)}\right\}+\mathcal{P}_{1}^{+}\left\{\left|\psi_{6}^{1}\right\rangle_{(0,1)}+q_{1}^{+} \lambda_{12}^{+} \tilde{\gamma}\left|\psi_{7}^{1}\right\rangle_{(0)}+\eta_{1}^{+} \lambda_{12}^{+}\left|\psi_{8}^{1}\right\rangle_{(0)}\right\} \\
& +p_{2}^{+} \tilde{\gamma}\left|\psi_{9}^{1}\right\rangle_{(1,0)}+\mathcal{P}_{2}^{+}\left|\psi_{10}^{1}\right\rangle_{(1,0)}+\mathcal{P}_{11}^{+}\left|\psi_{11}^{1}\right\rangle_{(-1,1)}+\mathcal{P}_{12}^{+}\left|\psi_{12}^{1}\right\rangle_{(0)} \\
& \left.+\lambda_{12}^{+}\left|\psi_{13}^{1}\right\rangle_{(2,0)}\right)+\mathcal{P}_{1}^{+}\left(p_{2}^{+}\left|\psi_{14}^{1}\right\rangle_{(1,0)}+\mathcal{P}_{2}^{+} \tilde{\gamma}\left|\psi_{15}^{1}\right\rangle_{(1,0)}+\mathcal{P}_{11}^{+} \tilde{\gamma}\left|\psi_{16}^{1}\right\rangle_{(-1,1)}\right. \\
& \left.+\mathcal{P}_{12}^{+} \tilde{\gamma}\left|\psi_{17}^{1}\right\rangle_{(0)}+\lambda_{12}^{+} \tilde{\gamma}\left|\psi_{18}^{1}\right\rangle_{(2,0)}\right)+p_{2}^{+} \mathcal{P}_{11}^{+}\left|\psi_{19}^{1}\right\rangle_{(0)}+\mathcal{P}_{2}^{+} \mathcal{P}_{11}^{+} \tilde{\gamma}\left|\psi_{20}^{1}\right\rangle_{(0)} \\
& +\mathcal{P}_{11}^{+} \lambda_{12}^{+} \tilde{\gamma}\left|\psi_{21}^{1}\right\rangle_{(1,0)},
\end{aligned}
$$

where the ghost-independent vectors $\left|\psi_{n}^{1}\right\rangle_{(0)},\left|\psi_{p}^{(1)}\right\rangle_{(1,0)},\left|\psi_{r}^{(1)}\right\rangle_{(-1,1)},\left|\psi^{(1) 1}\right\rangle_{(0,1)},\left|\psi_{t}^{(1) 1}\right\rangle_{(2,0)}$ for $n=$ $2-5,7,8,12,17,19,20, p=9,10,14,15,21, r=11,16, o=1,6$ and $t=13,18$ in $\mathcal{H} \otimes \mathcal{H}^{\prime}$ have the same decomposition and the properties as those in Eqs. (D.8), (D.9), whereas the ghostindependent vectors in Eq. (D.10), different from those in Eqs. (D.4), (D.5), (D.8), (D.9) are decomposed as follows:

$$
\begin{aligned}
\left|\psi_{n}^{0}\right\rangle_{(1,1)} & =a_{1}^{+\mu}\left(a_{1}^{+\nu} d_{12}^{+}|0\rangle \psi_{n \mid \mu \nu}^{0}+f_{1}^{+} d_{12}^{+} \tilde{\gamma}|0\rangle \psi_{n \mid \mu}^{\prime 0}+a_{2}^{+\nu}|0\rangle \psi_{n \mid \mu, \nu}^{0}+f_{2}^{+} \tilde{\gamma}|0\rangle \psi_{n \mid \mu}^{\prime \prime 0}\right) \\
& +f_{1}^{+} a_{2}^{+\nu} \tilde{\gamma}|0\rangle \psi_{n \mid \mu}^{0}+b_{11}^{+} d_{12}^{+}|0\rangle \psi_{n}^{\prime 0}+b_{12}^{+}|0\rangle \psi_{n}^{\prime \prime 0}+f_{1}^{+} f_{2}^{+}|0\rangle \psi_{n}^{0}, \\
\left|\psi_{35}^{0}\right\rangle_{(3,0)} & =a_{1}^{+\mu}\left(a_{1}^{+\nu} a_{1}^{+\rho}|0\rangle \psi_{35 \mid \mu \nu \rho}^{0}+a_{1}^{+\nu} f_{1}^{+} \tilde{\gamma}|0\rangle \psi_{35 \mid \mu \nu}^{0}+b_{11}^{+}|0\rangle \psi_{35 \mid \mu}^{0}\right)+f_{1}^{+} b_{11}^{+} \tilde{\gamma}|0\rangle \psi_{35}^{0},
\end{aligned}
$$

for $n=1,26$.

Finally, conditions (6.5), (6.6), as applied to $(n)_{2}=(2,1)$, permit one to decompose the fermionic field vectors $\left|\chi_{0}^{l}\right\rangle_{(s)_{3}}$ derived from the general Eq.(5.8), in even (for $l=0$ ) and odd (for $l=1$ ) powers of ghosts, consisting of $(39+35)$ summands, and starting from the ghost-independent vector $|\Psi\rangle_{(2,1)}$ (from the first order in ghost momenta $\mathcal{P}_{I}$ for $l=1$ ),

$$
\begin{aligned}
\left|\chi_{0}^{0}\right\rangle_{(2,1)}= & |\Psi\rangle_{(2,1)}+p_{1}^{+}\left(q_{1}^{+}\left|\psi_{1}\right\rangle_{(0,1)}+\eta_{1}^{+} \tilde{\gamma}\left|\psi_{2}\right\rangle_{(0,1)}+q_{2}^{+}\left|\psi_{3}\right\rangle_{(1,0)}+\eta_{2}^{+} \tilde{\gamma}\left|\psi_{4}\right\rangle_{(1,0)}\right. \\
& +\eta_{11}^{+} \tilde{\gamma}\left|\psi_{5}\right\rangle_{(-1,1)}+\eta_{12}^{+} \tilde{\gamma}\left|\psi_{6}\right\rangle_{(0)}+\vartheta_{12}^{+} \tilde{\gamma}\left|\psi_{7}\right\rangle_{(2,0)}+p_{1}^{+}\left\{q_{1}^{+} \vartheta_{12}^{+} \tilde{\gamma}\left|\psi_{8}\right\rangle_{(0)}\right. \\
& \left.+\eta_{1}^{+} \vartheta_{12}^{+}\left|\psi_{9}\right\rangle_{(0)}\right\}+\mathcal{P}_{1}^{+}\left\{q_{1}^{+} \vartheta_{12}^{+}\left|\psi_{10}\right\rangle_{(0)}+\eta_{1}^{+} \vartheta_{12}^{+} \tilde{\gamma}\left|\psi_{11}\right\rangle_{(0)}\right\}+\lambda_{12}^{+}\left\{\left(q_{1}^{+}\right)^{2} \tilde{\gamma}\left|\psi_{12}\right\rangle_{(0)}\right. \\
& \left.\left.+q_{1}^{+} \eta_{1}^{+}\left|\psi_{13}\right\rangle_{(0)}\right\}\right)+\mathcal{P}_{1}^{+}\left(q_{1}^{+} \tilde{\gamma}\left|\psi_{14}\right\rangle_{(0,1)}+\eta_{1}^{+}\left|\psi_{15}\right\rangle_{(0,1)}+q_{2}^{+} \tilde{\gamma}\left|\psi_{16}\right\rangle_{(1,0)}\right. \\
& +\eta_{2}^{+}\left|\psi_{17}\right\rangle_{(1,0)}+\eta_{11}^{+}\left|\psi_{18}\right\rangle_{(-1,1)}+\eta_{12}^{+}\left|\psi_{19}\right\rangle_{(0)}+\vartheta_{12}^{+}\left|\psi_{20}\right\rangle_{(2,0)} \\
& \left.+\lambda_{12}^{+}\left\{\left(q_{1}^{+}\right)^{2}\left|\psi_{21}\right\rangle_{(0)}+q_{1}^{+} \eta_{1}^{+} \tilde{\gamma}\left|\psi_{22}\right\rangle_{(0)}\right\}\right)+p_{2}^{+}\left(q_{1}^{+}\left|\psi_{23}\right\rangle_{(1,0)}+\eta_{1}^{+} \tilde{\gamma}\left|\psi_{24}\right\rangle_{(1,0)}\right. \\
& \left.+\eta_{11}^{+} \tilde{\gamma}\left|\psi_{25}\right\rangle_{(0)}\right)+\mathcal{P}_{2}^{+}\left(q_{1}^{+} \tilde{\gamma}\left|\psi_{26}\right\rangle_{(1,0)}+\eta_{1}^{+}\left|\psi_{27}\right\rangle_{(1,0)}+\eta_{11}^{+}\left|\psi_{28}\right\rangle_{(0)}\right) \\
& +\mathcal{P}_{11}^{+}\left(q_{1}^{+} \tilde{\gamma}\left|\psi_{29}\right\rangle_{(-1,1)}+\eta_{1}^{+}\left|\psi_{30}\right\rangle_{(-1,1)}+q_{2}^{+} \tilde{\gamma}\left|\psi_{31}\right\rangle_{(0)}+\eta_{2}^{+}\left|\psi_{32}\right\rangle_{(0)}\right. \\
& \left.+\vartheta_{12}^{+}\left|\psi_{33}\right\rangle_{(1,0)}\right)+\mathcal{P}_{12}^{+}\left(q_{1}^{+} \tilde{\gamma}\left|\psi_{34}\right\rangle_{(0)}+\eta_{1}^{+}\left|\psi_{35}\right\rangle_{(0)}\right)+\lambda_{12}^{+}\left(q_{1}^{+} \tilde{\gamma}\left|\psi_{36}\right\rangle_{(2,0)}\right. \\
& \left.+\eta_{1}^{+}\left|\psi_{37}\right\rangle_{(2,0)}+\eta_{11}^{+}\left|\psi_{38}\right\rangle_{(1,0)}\right)
\end{aligned}
$$




$$
\begin{aligned}
\left|\chi_{0}^{1}\right\rangle_{(2,1)}= & p_{1}^{+}\left(\left|\varphi_{1}\right\rangle_{(1,1)}+p_{1}^{+}\left\{q_{1}^{+}\left|\varphi_{2}\right\rangle_{(-1,1)}+\eta_{1}^{+} \tilde{\gamma}\left|\varphi_{3}\right\rangle_{(-1,1)}+q_{2}^{+}\left|\varphi_{4}\right\rangle_{(0)}+\eta_{2}^{+} \tilde{\gamma}\left|\varphi_{5}\right\rangle_{(0)}\right.\right. \\
& \left.+\vartheta_{12}^{+} \tilde{\gamma}\left|\varphi_{6}\right\rangle_{(1,0)}\right\}+\mathcal{P}_{1}^{+}\left\{q_{1}^{+} \tilde{\gamma}\left|\varphi_{7}\right\rangle_{(-1,1)}+\eta_{1}^{+}\left|\varphi_{8}\right\rangle_{(-1,1)}+q_{2}^{+} \tilde{\gamma}\left|\varphi_{9}\right\rangle_{(0)}\right. \\
& \left.+\eta_{2}^{+}\left|\varphi_{10}\right\rangle_{(0)}+\vartheta_{12}^{+}\left|\varphi_{11}\right\rangle_{(1,0)}\right\}+p_{2}^{+}\left\{q_{1}^{+}\left|\varphi_{12}\right\rangle_{(0)}+\eta_{1}^{+} \tilde{\gamma}\left|\varphi_{13}\right\rangle_{(0)}\right\}+\mathcal{P}_{2}^{+}\left\{q_{1}^{+} \tilde{\gamma}\left|\varphi_{14}\right\rangle_{(0)}\right. \\
& \left.+\eta_{1}^{+}\left|\varphi_{15}\right\rangle_{(0)}\right\}+\mathcal{P}_{11}^{+} \vartheta_{12}^{+}\left|\varphi_{16}\right\rangle_{(0)}+\lambda_{12}^{+}\left\{q_{1}^{+} \tilde{\gamma}\left|\varphi_{17}\right\rangle_{(1,0)}+\eta_{1}^{+}\left|\varphi_{18}\right\rangle_{(1,0)}\right. \\
& \left.\left.+\eta_{11}^{+}\left|\varphi_{19}\right\rangle_{(0)}\right\}\right)+p_{2}^{+}\left(\left|\varphi_{20}\right\rangle_{(2,0)}+\mathcal{P}_{1}^{+}\left\{q_{1}^{+} \tilde{\gamma}\left|\varphi_{21}\right\rangle_{(0)}+\eta_{1}^{+}\left|\varphi_{22}\right\rangle_{(0)}\right\}\right)+\mathcal{P}_{2}^{+}\left(\tilde{\gamma}\left|\varphi_{23}\right\rangle_{(2,0)}\right. \\
& \left.+\mathcal{P}_{1}^{+}\left\{q_{1}^{+}\left|\varphi_{24}\right\rangle_{(0)}+\eta_{1}^{+} \tilde{\gamma}\left|\varphi_{25}\right\rangle_{(0)}\right\}\right)+\mathcal{P}_{1}^{+}\left(\tilde{\gamma}\left|\varphi_{26}\right\rangle_{(1,1)}+\mathcal{P}_{11}^{+} \vartheta_{12}^{+}\left|\varphi_{27}\right\rangle_{(0)}\right. \\
& \left.+\lambda_{12}^{+}\left\{q_{1}^{+}\left|\varphi_{28}\right\rangle_{(1,0)}+\eta_{1}^{+} \tilde{\gamma}\left|\varphi_{29}\right\rangle_{(1,0)}+\eta_{11}^{+} \tilde{\gamma}\left|\varphi_{30}\right\rangle_{(0)}\right\}\right)+\mathcal{P}_{11}^{+}\left(\tilde{\gamma}\left|\varphi_{31}\right\rangle_{(0,1)}\right. \\
& \left.+\lambda_{12}^{+}\left\{q_{1}^{+}\left|\varphi_{32}\right\rangle_{(0)}+\eta_{1}^{+} \tilde{\gamma}\left|\varphi_{33}\right\rangle_{(0)}\right\}\right)+\mathcal{P}_{12}^{+} \tilde{\gamma}\left|\varphi_{34}\right\rangle_{(1,0)}+\lambda_{12}^{+} \tilde{\gamma}\left|\varphi_{35}\right\rangle_{(3,0)}
\end{aligned}
$$

In Eqs. (D.15), the ghost-independent vectors $\left|\varphi_{n}\right\rangle_{(\ldots)}, n=1, \ldots, 35$ have the same decomposition and properties as $\left|\psi_{n}^{0}\right\rangle_{(\ldots)}$ in Eqs. (D.10) (D.12), (D.13), whereas the ghost-independent vector in Eq. (D.14), different from the remaining ones, is the vector $|\Psi\rangle_{(2,1)}$, which reads

$$
\begin{aligned}
|\Psi\rangle_{(2,1)} & =a_{1}^{+\mu}\left(a_{1}^{+\nu} a_{1}^{+\rho} d_{12}^{+}|0\rangle \psi_{\mu \nu \rho}+a_{1}^{+\nu} a_{2}^{+\rho}|0\rangle \Psi_{\mu \nu, \rho}+a_{1}^{+\nu} f_{1}^{+} d_{12}^{+} \tilde{\gamma}|0\rangle \psi_{\mu \nu}+a_{1}^{+\nu} f_{2}^{+} \tilde{\gamma}|0\rangle \psi_{\mu \nu}^{\prime}\right. \\
& \left.+f_{1}^{+} a_{2}^{+\nu} \tilde{\gamma}|0\rangle \psi_{\mu, \nu}^{\prime}+f_{1}^{+} f_{2}^{+}|0\rangle \psi_{\mu}+b_{11}^{+} d_{12}^{+}|0\rangle \psi_{\mu}^{\prime}+b_{12}^{+}|0\rangle \psi_{\mu}^{\prime \prime}\right) \\
& +b_{11}^{+}\left(a_{2}^{+\mu}|0\rangle \psi_{\mu}^{\prime \prime \prime}+f_{1}^{+} d_{12}^{+} \tilde{\gamma}|0\rangle \psi+f_{2}^{+} \tilde{\gamma}|0\rangle \psi^{\prime}\right)+b_{12}^{+} f_{1}^{+} \tilde{\gamma}|0\rangle \psi^{\prime \prime}
\end{aligned}
$$

with the initial spin-tensor field $\Psi_{\mu \nu, \rho}$, describing a massless particle with spin $\left(\frac{5}{2}, \frac{3}{2}\right)$.

\section{References}

[1] M. Vasiliev, Higher spin gauge theories in various dimensions, Fortsch. Phys. 52 (2004) 702-717, arXiv:hep-th/0401177]; D. Sorokin, Introduction to the classical theory of higher spins, AIP Conf. Proc. 767 (2005) 172-202, arXiv:hep-th/0405069]; N. Bouatta, G. Compère, A. Sagnotti, An introduction to free higher-spin fields, [arXiv:hep-th/0409068]; A. Sagnotti, E. Sezgin, P. Sundell, On higher spins with a strong $\operatorname{Sp}(2, R)$ sondition, arXiv:hep-th/0501156]; X. Bekaert, S. Cnockaert, C. Iazeolla, M.A. Vasiliev, Nonlinear higher spin theories in various dimensions, arXiv:hep-th/0503128]; A. Fotopoulos, M. Tsulaia, Gauge Invariant Lagrangians for Free and Interacting Higher Spin Fields. A review of BRST formulation, Int.J.Mod.Phys. A24 (2008) 1-60, [arXiv:0805.1346[hep-th]].

[2] M. Fierz, W. Pauli, On relativistic wave equations for particles of arbitrary spin in an electromagnetic field, Proc. R. Soc. London, Ser. A, 173 (1939) 211-232.

[3] L.P.S. Singh, C.R. Hagen, Lagrangian formulation for arbitrary spin. 1. The bosonic case, Phys. Rev. D9 (1974) 898-909; Lagrangian formulation for arbitrary spin. 2. The fermionic case, Phys. Rev. D9 (1974) 910-920. 
[4] C. Fronsdal, Massless fields with integer spin, Phys. Rev. D18 (1978) 3624-3629; J. Fang, C. Fronsdal, Massless fileds with half-integral spin, Phys. Rev. D18 (1978) 3630-3633; C. Fronsdal, Singletons and massless, integer-spin fileds on de Sitter space, Phys. Rev. D20 (1979) 848-856; J. Fang, C. Fronsdal, Massless, half-integer-spin fields in de Sitter space, Phys. Rev. D22 (1980) 1361-1367; M.A. Vasiliev, 'Gauge' Form Of Description Of Massless Fields With Arbitrary Spin (in Russian), Yad.Fiz. 32 (1980) 855-861.

[5] N. Beisert, M. Bianchi, J.F. Morales, H. Samtleben, Higher spin symmetries and N=4 SYM, JHEP 0407 (2004) 058, arXiv:hep-th/0405057]; A.C. Petkou, Holography, duality and higher spin fields, arXiv:hep-th/0410116]; P.J. Heslop, F. Riccioni, On the fermionic Grande Bouffe: more on higher spin symmetry breaking in AdS/CFT, JHEP 0510 (2005) 060, arXiv:hep-th/0508086]; M. Bianchi, V. Didenko, Massive higher spin multiplets and holography, arXiv:hep-th/0502220].

[6] S. Deser, A. Waldron, Gauge invariances and phases of massive higher spins in (A)dS, Phys. Rev. Lett. 87 (2001) 031601, |arXiv:hep-th/0102166|; S. Deser, A. Waldron, Partial Masslessness of Higher Spins in (A)dS, Nucl. Phys. B607 (2001) 577-604, arXiv:hep-th/0103198; K. Hallowell, A. Waldron, Constant curvature algebras and higher spin action generating functions, Nucl. Phys. B724 (2005) 453-486, arXiv:hep-th/0505255]; E.D. Skvortsov, M.A. Vasiliev, Geometric formulation for partially massless fields, Nucl. Phys. B756 (2006) 117-147, arXiv:hep-th/0601095].

[7] G. Bonelli, On the boundary gauge dual of closed tensionless free strings in AdS, JHEP 0411 (2004) 059, arXiv:hep-th/0407144]; On the tensionless limit of bosonic strings, infinite symmetries and higher spins, Nucl. Phys. B669 (2003) 159-172, arXiv:hep-th/0305155.

[8] G. Barnich, M. Grigoriev, A. Semikhatov, I. Tipunin, Parent field theory and unfolding in BRST first-quantized terms, Commun. Math. Phys. 260 (2005) 141; G. Barnich, M. Grigoriev, Parent form for higher spin fields in anti-de Sitter space, JHEP 0608 (2006) 013; M. Grigoriev, Off-shell gauge fields from BRST quantization, arXiv:hep-th/0605089|; O.A. Gelfond, M.A. Vasiliev, Unfolding versus BRST and currents in $S p(2 M)$ invariant higher-spin theory, arXiv:1001.2585[hep-th]].

[9] K.B. Alkalaev, M. Grigoriev, I.Y. Tipunin, Massless Poincare modules and gauge invariant equations, Nucl. Phys. B823 (2009) 509, arXiv:0811.3999 [hep-th]]; K.B. Alkalaev, M. Grigoriev, Unified BRST description of AdS gauge fields, Nucl. Phys. B835 (2010) 197, arXiv:0910.2690[hep-th]]; K. Alkalaev, Mixed-symmetry tensor conserved currents and AdS/CFT correspondence, arXiv:1207.1079 [hep-th]].

[10] M.A. Vasiliev, Cubic interactions of bosonic higher spin gauge fields in AdS(5), Nucl. Phys. B616 (2001) 106-162 [Erratum-ibid. B 652 (2003) 407], |arXiv:hep-th/0106200|; Higher Spin Superalgebras in any Dimension and their Representations, JHEP 12 (2004) 046, [hep-th/0404124]; Holography, Unfolding and Higher-Spin Theory, arXiv:1203.5554[hep-th]].

[11] V.E. Didenko, E.D. Skvortsov, Towards higher-spin holography in ambient space of any dimension, arXiv:1207.6786[hep-th]]; Exact higher-spin symmetry in CFT: all correlators in unbroken Vasiliev theory, arXiv:1210.7963[hep-th]].

[12] M. Porrati, Universal limits of massless higher-spin particles, Phys.Rev. D78 (2008) 065016; M. Porrati, R. Rahman, A model independent ultraviolet cutoff for theories with charged massive higher spin fileds, Nucl.Phys. B814 (2009) 370; M. Porrati, R. Rahman, Causal propagation of a charged spin $3 / 2$ field in an external electromagnetic background, Phys.Rev. D80 
(2009) 025009 arXiv:0906.1432[hep-th]]; M. Porrati, R. Rahman, A. Sagnotti, String Theory and The Velo-Zwanziger Problem, arXiv:1011.6411[hep-th]].

[13] M. Henneaux, G.L. Gomez, R. Rahman, Higher-Spin Fermionic Gauge Fields and Their Electromagnetic Coupling, JHEP 1208 (2012) 093, arXiv:1206.1048[hep-th]].

[14] A. Sagnotti and M. Taronna, String Lessons for Higher-Spin Interactions, Nucl. Phys. B842 (2011) 299, arXiv:1006.5242[hep-th]]; E. Joung, M. Taronna, Cubic interactions of massless higher spins in (A)dS: metric-like approach, arXiv:1110.5918[hep-th]].

[15] R.R. Metsaev, Shadows, currents and AdS, Phys.Rev. D 78 (2008) 106010, [arXiv:0805. 3472[hep-th]]; CFT adapted gauge invariant formulation of arbitrary spin fields in AdS and modified de Donder gauge, Phys.Lett. B671 (2009) 128-134, arXiv:0808.3945[hep-th]]; CFT adapted gauge invariant formulation of massive arbitrary spin fields in AdS, Phys.Lett. B682 (2010) 455-461, arXiv:0907.2207[hep-th]]; Gauge invariant approach to low-spin anomalous conformal currents and shadow fields, Phys.Rev. D83 (2011) 106004, arXiv:1011.4261 [hepth]]; Extended Hamiltonian Action for Arbitrary Spin Fields in Flat And AdS Spaces, arXiv:1112.0976[hep-th]].

[16] Yu.A. Zinoviev, Frame-like gauge invariant formulation for massive high spin particles, Nucl. Phys. B808 (2009) 185, arXiv:0808.1778[hep-th]]; Yu.M. Zinoviev, Gravitational cubic interactions for a massive mixed symmetry gauge field, arXiv:1107.3222[hep-th]]; On electromagnetic interactions for massive mixed symmetry field, JHEP 1103 (2011) 082, arXiv:1012.2706 [hep-th]]; N. Boulanger, E.D. Skvortsov, Yu.M. Zinoviev, Gravitational cubic interactions for a simple mixed-symmetry gauge field in AdS and flat backgrounds, J.Phys.A A44 (2011) 415403, arXiv:1107.1872[hep-th]]; I.L. Buchbinder, T.V. Snegirev, Yu.M. Zinoviev, Cubic interaction vertex of higher-spin fields with external electromagnetic field, Nucl.Phys. B 864 (2012) 694-721, arXiv:1204.2341[hep-th]]; Gauge invariant Lagrangian formulation of massive higher spin fields in (A)dS 3 space, Phys.Lett. B716 (2012) 243-248, [arXiv:1207.1215[hep-th]].

[17] I.L. Buchbinder, A.V. Galajinsky, V.A. Krykhtin, Quartet unconstrained formulation for massless higher spin fields, Nucl. Phys. B779 (2007) 155, [arXiv: hep-th/0702161]; I.L. Buchbinder, A.V. Galajinsky, Quartet unconstrained formulation for massive higher spin fields, JHEP 0811 (2008) 081 [arXiv:0810.2852[hep-th]].

[18] D.P. Sorokin, M.A. Vasiliev, Reducible higher-spin multiplets in flat and AdS spaces and their geometric frame-like formulation, Nucl.Phys. B809 (2009) 110-157, [arXiv:0807.0206[hep-th]].

[19] D. Francia, Geometric Lagrangians for massive higher-spin fields, Nucl. Phys. B 796 (2008) 77, arXiv:0710.5378 [hep-th]].

[20] N. Boulanger, P. Sundell, An action principle for Vasiliev's four-dimensional higher-spin gravity, arXiv:1102.2219 [hep-th]]; N. Doroud, L. Smolin, An action for higher spin gauge theory in four dimensions, arXiv:1102.3297[hep-th]].

[21] M.A. Vasiliev, Free massless fermionic fields of arbitrary spin in D-dimensional anti-de Sitter space, Nucl. Phys. B301 (1988) 26-51; V.E. Lopatin, M.A. Vasiliev, Free massless bosonic fields of arbitrary spin in D-dimensional de Sitter space, Mod. Phys. Lett. A3 (1998) 257-265.

[22] Yu.M. Zinoviev, On massive high spin particles in AdS, arXiv:hep-th/0108192]; R.R. Metsaev, Massive totally symmetric fields in AdS(d), Phys.Lett. B590 (2004) 95-104, [arXiv:hep-th/0312297]; Fermionic fields in the d-dimensional anti-de Sitter spacetime, Phys. 
Lett. B419 (1998) 49-56, arXiv:hep-th/9802097]; Light-cone form of field dynamics in anti-de Sitter space-time and AdS/CFT correspondence, Nucl. Phys. B563 (1999) 295-348, arXiv:hep-th/9906217]; Massless arbitrary spin fields in AdS(5) Phys. Lett. B531 (2002) 152160, |arXiv:hep-th/0201226]; P. de Medeiros, Massive gauge-invariant field theories on space of constant curvature, Class. Quant. Grav. 21 (2004) 2571-2593, arXiv:hep-th/0311254.

[23] J.M.F. Labastida, T.R. Morris, Massless mixed symmetry bosonic free fields, Phys. Lett. B180 (1986) 101-106; J.M.F. Labastida, Massless fermionic free fields, Phys. Lett. B186 (1987) 365-369; Massless bosonic free fields, Phys. Rev. Lett. 58 (1987) 531-534; Massless particles in arbitrary representations of the Lorentz group, Nucl. Phys. B322 (1989) 185-209.

[24] L. Brink, R.R. Metsaev, M.A. Vasiliev, How massless are massless fields in $A d S_{d}$, Nucl. Phys. B586 (2000) 183-205, arXiv:hep-th/0005136.

[25] R.R. Metsaev, Massless mixed symmetry bosonic free fields in d-dimensional anti-de Sitter space-time, Phys. Lett. B354 (1995) 78-84; Mixed-symmetry massive fields in AdS(5), Class. Quant. Grav. 22 (2005) 2777-2796, arXiv:hep-th/0412311.

[26] T. Curtright, Massless field supermultiplets with arbitrary spin, Phys. Lett. B85 (1979) 219-224; Generalized gauge fields Phys. Lett. B165 (1985) 304-308.

[27] A. Campoleoni, D. Francia, J. Mourad, A. Sagnotti, Unconstrained Higher Spins of Mixed Symmetry. II. Fermi Fields. Nucl.Phys. B828 (2010) 405-514, arXiv:0904.4447[hep-th]].

[28] A. Campoleoni, D. Francia, J. Mourad, A. Sagnotti, Unconstrained Higher Spins of Mixed Symmetry. I. Bose Fields. Nucl.Phys. B815 (2009) 289-357, arXiv:0810.4350[hep-th]]

[29] E.D. Skvortsov, Yu.M. Zinoviev, Frame-like Actions for Massless Mixed-Symmetry Fields in Minkowski space. Fermions, Nucl.Phys. B843 (2011) 559-569, arXiv:1007.4944[hep-th]].

[30] Yu.M. Zinoviev, Frame-like gauge invariant formulation for mixed symmetry fermionic fields, Nucl.Phys. B821 (2009) 21-47, [arXiv:0904.0549[hep-th]].

[31] I.L. Buchbinder and A. Reshetnyak, General Lagrangian Formulation for Higher Spin Fields with Arbitrary Index Symmetry. I. Bosonic fields, Nucl. Phys. B 862 (2012) 270-327, arXiv:1110.5044[hep-th]].

[32] A.A. Reshetnyak, On Lagrangian formulations for arbitrary bosonic HS fields on Minkowski backgrounds, Phys. of Particles and Nuclei 43 (2012) 689-693, arXiv:1202.4710[hep-th]].

[33] A. Pashnev, M.M. Tsulaia, Description of the higher massless irreducible integer spins in the BRST approach, Mod. Phys. Lett. A13 (1998) 1853-1864, arXiv:hep-th/9803207].

[34] X. Bekaert, N. Boulanger, Tensor gauge fields in arbitrary representations of GL(D,R): duality and Poincare lemma, Commun. Math. Phys. 245 (2004) 27-67, arXiv:hep-th/0208058; On geometric equations and duality for free higher spins, Phys. Lett. B561 (2003) 183-190, arXiv:hep-th/0301243]; Mixed symmetry gauge fields in a flat background, arXiv:hep-th/0310209]; Tensor gauge fields in arbitrary representations of GL(D,R). II. Quadratic actions, Commun. Math. Phys. 271 (2007) 723-773, arXiv:hep-th/0606198]; X. Bekaert, N. Boulanger, S. Cnockaert, No self-interaction for two-column massless fields, J. Math. Phys. 46 (2005) 012303, arXiv:hep-th/0407102].

[35] P. de Medeiros, C. Hull, Geometric second order field equations for general tensor gauge fields, JHEP 0305 (2003) 019, arXiv:hep-th/0303036]. 
[36] E.S. Fradkin, G.A. Vilkovisky, Quantization of relativistic systems with constraints, Phys. Lett. B55 (1975) 224-226; I.A. Batalin, G.A. Vilkovisky, Relativistic S-matrix of dynamical systems with boson and fermion constraints, Phys. Lett. B69 (1977) 309-312; I.A. Batalin, E.S. Fradkin, Operator quantization of relativistic dynamical systems subject to first class constraints, Phys. Lett. B128 (1983) 303.

[37] I.A. Batalin, E.S. Fradkin, Operator quantization method and abelization of dynamical systems subject to first class constraints, Riv. Nuovo Cimento, 9, No. 10 (1986) 1; I.A. Batalin, E.S. Fradkin, Operator quantization of dynamical systems subject to constraints. A further study of the construction, Ann. Inst. H. Poincare, A49 (1988) 145-214.

[38] M. Henneaux, Hamiltonian form of the path integral for theories with a gauge freedom, Phys. Rept. 126 (1985) 1-66; M. Henneaux, C. Teitelboim, Quantization of gauge systems, Princeton Univ. Press, 1992.

[39] M. Alexandrov, M. Kontsevich, A.Schwarz, O. Zaboronsky, The geometry of the master equation and topological quantum field theory, Int. I. Mod. Phys. A12 (1997) 1405-1430, arXiv:hep-th/9502010.

[40] M. Grigoriev, P.H. Damgaard, Superfield BRST charge and the master action, Phys. Lett. B474 (2000) 323-330, arXiv:hep-th/9911092; G. Barnich, M. Grigoriev, Hamiltonian BRST and Batalin-Vilkovisky formalisms for second quantization of gauge theories, Commun. Math. Phys. 254 (2005) 581-601, arXiv:hep-th/0310083.

[41] D.M. Gitman, P.Yu. Moshin, A.A. Reshetnyak, Local superfield Lagrangian BRST quantization, J. Math. Phys. 46 (2005) 072302-01-072302-24, |arXiv:hep-th/0507160|; An embedding of the BV quantization into an $\mathrm{N}=1$ local superfield formalism, Phys. Lett. B 621 (2005) 295-308, arXiv:hep-th/0507049].

[42] D. Francia, A. Sagnotti, Free geometric equations for higher spins, Phys. Lett. B543 (2002) 303-310, arXiv:hep-th/0207002; A. Sagnotti, M. Tsulaia, On higher spins and the tensionless limit of String Theory, Nucl. Phys. B682 (2004) 83-116, arXiv:hep-th/0311257]; D. Francia, J. Mourad, A. Sagnotti, Current exchanges and unconstrained higher spins, Nucl. Phys. B773 (2007) 203 arXiv:hep-th/0701163.

[43] Y.M. Zinoviev, On massive mixed symmetry tensor fields in Minkowski space and (A)dS, arXiv:hep-th/0211233]; First order formalism for mixed symmetry tensor fields, [arXiv:hep-th/0304067]; First order formalism for massive mixed symmetry tensor fields in Minkowski and (A)dS spaces, arXiv:hep-th/0306292.

[44] C.S. Aulakh, I.G. Koh, S. Ouvry, Higher spin fields with mixed symmetry, Phys. Lett. B173 (1986) 284-288; S. Ouvry, J. Stern, Gauge fields of any spin and symmetry, Phys. Lett. B177 (1986) 335-340; A.K.H. Bengtsson, A unified action for higher spin gauge bosons from covariant string theory, Phys. Lett. B182 (1986) 321-325.

[45] R.R.Metsaev, Free totally (anti)symmetric massless fermionic fields in d-dimensional anti-de Sitter space, Class. Quant. Grav. 14 (1997) L115-L121, |arXiv:hep-th/9707066]; Fermionic fields in the d-dimensional anti-de Sitter spacetime, Phys. Lett. B419 (1998) 49-56, arXiv:hep-th/9802097]; Arbitrary spin massless bosonic fields in d-dimensional anti-de Sitter space, |arXiv:hep-th/9810231]; Light-cone form of field dynamics in antide Sitter space-time and AdS/CFT correspondence, Nucl. Phys. B563 (1999) 295-348, arXiv:hep-th/9906217]; Massless arbitrary spin fields in AdS(5) Phys. Lett. B531 (2002) 
152-160, arXiv:hep-th/0201226]; Massive totally symmetric fields in AdS(d), Phys. Lett. B590 (2004) 95-104, |arXiv:hep-th/0312297|; R.R. Metsaev, Mixed-symmetry massive fields in $\operatorname{AdS}(5)$, Class. Quant. Grav. 22 (2005) 2777-2796, arXiv:hep-th/0412311]; Cubic interaction vertices of massive and massless higher spin fields, Nucl. Phys. B759 (2006) 147-201, arXiv:hep-th/0512342 ; R.R. Metsaev, Gauge invariant formulation of massive totally symmetric fermionic fileds in (A)dS space, Phys. Lett. B643 (2006) 205-212, arXiv:hep-th/0609029].

[46] R.R. Metsaev, Massless mixed symmetry bosonic free fields in d-dimensional anti-de Sitter space-time, Phys. Lett. B354 (1995) 78-84; Fermionic fields in the d-dimensional anti-de Sitter spacetime, Phys. Lett. B419 (1998) 49-56, arXiv:hep-th/9802097.

[47] C. Burdik, A. Pashnev, M. Tsulaia, On the mixed symmetry irreducible representations of the Poincare group in the BRST approach, Mod. Phys. Lett. A16 (2001) 731-746, arXiv:hep-th/0101201]; The Lagrangian description of representations of the Poincare group, Nucl. Phys. Proc. Suppl. 102 (2001) 285-292, arXiv:hep-th/0103143].

[48] R.R. Metsaev, Cubic interaction vertices of massive and massless higher spin fields, Nucl. Phys. B759 (2006) 147-201, arXiv:hep-th/0512342.

[49] A. Fotopoulos, K.L. Panigrahi, M. Tsulaia, Lagrangian formulation of higher spin theories on AdS, Phys. Rev. D74 (2006) 085029, arXiv:hep-th/0607248]; A. Fotopoulos and M. Tsulaia, On the Tensionless Limit of String theory, Off - Shell Higher Spin Interaction Vertices and BCFW Recursion Relations, JHEP 1011 (2010) 086, arXiv:1009.0727[hep-th]]; P. Dempster and M. Tsulaia, On the Structure of Quartic Vertices for Massless Higher Spin Fields on Minkowski Background, Nucl.Phys. B 865 (2012) 353-375, arXiv:1203.5597[hep-th]].

[50] R. Manvelyan, K. Mkrtchyan, W. Ruehl, Off-shell construction of some trilinear higher spin gauge field interactions, Nucl.Phys. B826 (2010) 1-17, arXiv:0903.0243[hep-th]]; Direct construction of a cubic selfinteraction for higher spin gauge fields, Nucl.Phys. B844 (2011) 348-364, arXiv:1002.1358[hep-th]]; General trilinear interaction for arbitrary even higher spin gauge fields, Phys.Lett. B696 (2011) 410-415, arXiv:1009.1054[hep-th]]; Radial Reduction and Cubic Interaction for Higher Spins in (A)dS space, arXiv:1210.7227[hep-th]]; R. Manvelyan, K. Mkrtchyan, W. Ruehl, M. Tovmasyan, On Nonlinear Higher Spin Curvature, Phys.Lett. B699 (2011) 187-191, arXiv:1102.0306[hep-th]].

[51] K.B. Alkalaev, Free fermionic higher spin fields in AdS(5), Phys. Lett. B519 (2001) 121128, [arXiv: hep-th/0107040]; K.B. Alkalaev, M.A. Vasiliev, N=1 supersymmetric theory of higher spin gauge fields in AdS(5) at the cubic level, Nucl. Phys. B655 (2003) 57-92, arXiv:hep-th/0206068]; K.B. Alkalaev, Two column higher spin massless fields in AdS(d), Theor. Math. Phys. 140 (2004) 1253-1263, arXiv:hep-th/0311212; K.B. Alkalaev, O.V. Shaynkman, M.A. Vasiliev, On the frame-like formulation of mixed-symmetry massless fields in (A)dS(d), Nucl. Phys. B692 (2004) 363-393, arXiv:hep-th/0311164]; Lagrangian formulation for free mixed-symmetry bosonic gauge fields in (A)dS(d), JHEP 0508 (2005) 069, arXiv:hep-th/0501108]; Frame-like formulation for free mixed-symmetry bosonic massless higher-spin fields in AdS(d), arXiv:hep-th/0601225]; K.B. Alkalaev, Mixed-symmetry massless gauge fields in AdS(5), Theor. Math. Phys. 149 (2006) 1338-1348.

[52] Yu.M. Zinoviev, Massive N=1 supermultiplets with arbitrary superspins, arXiv:0704.1535]; Massive supermultiplets with spin 3/2, arXiv:hep-th/0703118 
[53] A. Fotopoulos, M. Tsulaia, Interacting higher spins and the high energy limit of the bosonic string, arXiv:0705.2939.

[54] A. Sagnotti, M. Tsulaia, On higher spins and the tensionless limit of string theory, Nucl. Phys. B682 (2004) 83-116, arXiv:hep-th/0311257.

[55] I.L. Buchbinder, V.A. Krykhtin, A. Pashnev, BRST approach to Lagrangian construction for fermionic massless higher spin fields, Nucl. Phys. B711 (2005) 367-391, arXiv:hep-th/0410215]; I.L. Buchbinder, V.A. Krykhtin, L.L. Ryskina, H. Takata, Gauge invariant Lagrangian construction for massive higher spin fermionic fields, Phys. Lett. B641 (2006) 386-392, arXiv:hep-th/0603212.

[56] I.L. Buchbinder, V.A. Krykhtin, A.A. Reshetnyak, BRST approach to Lagrangian construction for fermionic higher spin fields in AdS space, Nucl. Phys. B787 (2007) 211, arXiv:hep-th/0703049.

[57] I.L. Buchbinder, A. Pashnev, M. Tsulaia, Lagrangian formulation of the massless higher integer spin fields in the AdS background, Phys. Lett. B523 (2001) 338-346, arXiv:hep-th/0109067; X. Bekaert, I.L. Buchbinder, A. Pashnev, M. Tsulaia, On higher spin theory: strings, BRST, dimensional reductions, Class. Quant. Grav. 21 (2004) 14571464, arXiv:hep-th/0312252]; I.L. Buchbinder, V.A. Krykhtin, P.M. Lavrov, Gauge invariant Lagrangian formulation of higher massive bosonic field theory in AdS space, Nucl. Phys. B762 (2007) 344-376, arXiv:hep-th/0608005.

[58] C. Burdik, A. Reshetnyak, On representations of Higher Spin symmetry algebras for mixedsymmetry HS fields on AdS-spaces. Lagrangian formulation, J. Phys. Conf. Ser. 343 (2012) 012102, arXiv:1111.5516[hep-th]]

[59] Moshin P.Yu. and Reshetnyak A.A., BRST approach to Lagrangian formulation for mixedsymmetry fermionic higher-spin fields, JHEP. 10 (2007) 040, arXiv:0707.0386[hep-th]].

[60] I.L. Buchbinder, V.A. Krykhtin, L.L. Ryskina, Lagrangian formulation of massive fermionic totally antisymmetric tensor field theory in $\mathrm{AdS}_{d}$ space, Nucl. Phys. B819 (2009) 453-477, arXiv:0902.1471[hep-th]].

[61] R. Howe, Transcending classical invariant theory, J. Amer. Math. Soc. 3 (1989) 2; Remarks on classical invariant theory, Trans. Amer. Math. Soc. 2 (1989) 313.

[62] L.D. Faddeev, S.L. Shatashvili, Realization of the Schwinger term in the Gauss low and the possibility of correct quantization of a theory with anomalies, Phys.Lett. B167 (1986) 225238; I.A. Batalin, E.S. Fradkin, T.E. Fradkina, Another version for operatorial quantization of dynamical systems with iireducible constraints, Nucl. Phys. B314 (1989) 158-174; I.A. Batalin, I.V. Tyutin, Existence theorerm for the effective gauge algebra in the generalized canonical formalism and Abelian conversion of second class constraints, Int. J. Mod. Phys. A6 (1991) 3255-3282; E. Egorian, R. Manvelyan, Quantization of dynamical systems with first and second class constraints, Theor. Math. Phys. 94 (1993) 241-252.

[63] A. Kuleshov and A. Reshetnyak Programming realization of symbolic computations for nonlinear commutator superalgebras over the Heisenberg-Weyl superalgebra: data structures and processing methods, arXiv:0905.2705[hep-th]].

[64] C. Burdik, A. Pashnev, M. Tsulaia, Auxiliary representations of Lie algebras and the BRST constructions, Mod. Phys. Lett. A15 (2000) 281-291, arXiv:hep-th/0001195]. 
[65] W. Siegel, Gauging Ramond string fields via Osp(1,1/2), Nucl.Phys. B284 (1987) 632-644.

[66] W. Siegel, B. Zwiebach, Gauge string fields from the light cone, Nucl.Phys. B 282 (1987) 125-143.

[67] G. Barnich, M. Henneaux, Consistent couplings between fields with a gauge freedom and deformations of the master equation, Phys. Lett. B311 (1993) 123-129, arXiv:hep-th/9304057; M. Henneaux, Consistent interactions between gauge fields: the cohomological approach, Contemp. Math. 219 (1998) 93-105, arXiv:hep-th/9712226], M. Dubois-Violette, M. Henneaux, Generalized cohomology for irreducible tensor fields of mixed Young symmetry type, Lett. Math. Phys. 49 (1999) 245-252, arXiv:math.qa/9907135.

[68] D.-N. Verma, Structure of certain induced representations of complex semisimple Lie algebras, Yale Univ.,dissertation, 1966, Bull. Amer. Math. Soc. 74, No. 1 (1968) 160-166.

[69] I.N. Bernstein, I.M. Gelfand, S.I. Gelfand, The structure of representations by vectors of highest weight, Funct. Anal. i Prilozhen. 5 (1971) 1-9.

[70] J. Dixmier, Algebres enveloppantes, Gauthier-Villars, Paris (1974), English transl., Enveloping algebras, North-Holland, Amsterdam, 1977.

[71] A. Rocha-Caridi, Splitting criteria for $\mathfrak{p}$-modules induced from a parabolic and a BernsteinGelfand-Gelfand resolution of a finite-dimensional, irreducible $\mathfrak{p}$-module, Trans. Amer. Math. Soc., 262 (1980) 335-366;

A.J. Coleman, V.M. Futorny, Stratified L-modules, J. Algebra, 163 (1994) 219-234;

V. Futorny, V. Mazorchuk, Structure of $\alpha$-Stratified L-modules for finite-dimensional Lie algebras, J. Algebra, I 183 (1996) 456-482;

V. Mazorchuk, S. Ovsienko, Submodule structure of Generalized Verma modules induced from generic Gelfand-Zeitlin modules, Alg. and Repr. Theory 1 (1998) 3-26.

[72] C. Burdik, Realizations of the real simple Lie algebras: the method of construction, J. Phys. A: Math. Gen. 18 (1985) 3101-3112.

[73] V. G. Kac Infinite dimensional Lie algebras, Cambridge University Press, Cambridge (1990).

[74] C. Burdik, P. Grozman, D. Leites, A. Sergeev, Realization of Lie Algebras and Superalgebras in Terms of Creation and Annihilation Operators: I, Theor. Math. Phys. 124 (2000) 1048-58.

[75] V.K. Dobrev, Representations and characters of the Virasoro algebra and N=1 superVirasoro algebras, arXiv:0709.0105[hep-th]]. 